\title{
URN model of the requirements for operating and maintaining complex distributed systems
}

\author{
by \\ Mirela Tabra
}

A thesis submitted to the Faculty of Graduate and Postdoctoral Affairs

in partial fulfillment of the requirements for the degree of Master of Science in Information Systems Science

Department of Systems and Computer Engineering Carleton University

Ottawa, Ontario, Canada, K1S 5B6

January 2013

C Copyright 2013, Mirela Tabra 
Library and Archives

Canada

Published Heritage

Branch

395 Wellington Street

Ottawa ON K1A ON4

Canada
Bibliothèque et

Archives Canada

Direction du

Patrimoine de l'édition

395 , rue Wellington

Ottawa ON K1A ON4

Canada
Your file Votre référence

ISBN: 978-0-494-94307-6

Our file Notre référence

ISBN: $978-0-494-94307-6$
NOTICE:

The author has granted a nonexclusive license allowing Library and Archives Canada to reproduce, publish, archive, preserve, conserve, communicate to the public by telecommunication or on the Internet, loan, distrbute and sell theses worldwide, for commercial or noncommercial purposes, in microform, paper, electronic and/or any other formats.

The author retains copyright ownership and moral rights in this thesis. Neither the thesis nor substantial extracts from it may be printed or otherwise reproduced without the author's permission.
AVIS:

L'auteur a accordé une licence non exclusive permettant à la Bibliothèque et Archives Canada de reproduire, publier, archiver, sauvegarder, conserver, transmettre au public par télécommunication ou par l'Internet, prêter, distribuer et vendre des thèses partout dans le monde, à des fins commerciales ou autres, sur support microforme, papier, électronique et/ou autres formats.

L'auteur conserve la propriété du droit d'auteur et des droits moraux qui protege cette thèse. $\mathrm{Ni}$ la thèse ni des extraits substantiels de celle-ci ne doivent être imprimés ou autrement reproduits sans son autorisation.
In compliance with the Canadian Privacy Act some supporting forms may have been removed from this thesis.

While these forms may be included in the document page count, their removal does not represent any loss of content from the thesis.
Conformément à la loi canadienne sur la protection de la vie privée, quelques formulaires secondaires ont été enlevés de cette thèse.

Bien que ces formulaires aient inclus dans la pagination, il n'y aura aucun contenu manquant. 


\begin{abstract}
The objective of the thesis consists in modeling, understanding and evaluating how efficiently very complex distributed systems are operated and maintained. We consider that the systems are serving a large-scale organization, composed of sub-organizations which have distinct operational mandates, budgets, goals, managers and staff. We used the User Requirement Notation (URN), an international requirements engineering standard that integrates goal-oriented and scenario based modeling to model a subset of the requirements for the case study system. The requirements we focus on are concerned with the way the operation of the system is organized, how different departments involved in the system activities interact and what is the impact on the realization of their goals. During the construction of the model, an analysis and evaluation was done, allowing to understand some areas of improvement. A new version of the system, called the proposed system is designed, evaluated and compared with the working system.
\end{abstract}




\section{Acknowledgements}

My deepest gratitude goes to my supervisor Dr. Dorina Petriu for providing excellent guidance and support all along the way. It was great to receive detailed reviews and feedback on every chapter, and they have generated contributions to this thesis.

I would also like to thank my family for their understanding and support. 


\section{Table of Contents}

Abstract............................................................................................................................. ii

Acknowledgements .......................................................................................................................... iii

Table of Contents .................................................................................................................................. iv

List of Tables ....................................................................................................................................... vi

List of Figures........................................................................................................................ viii

List of Appendices................................................................................................................................. xiii

List of Acronyms .......................................................................................................................... xiv

1 Chapter: Introduction ........................................................................................................ 1

1.1 Objectives and Motivation ..................................................................................

1.2 Thesis contributions...............................................................................................

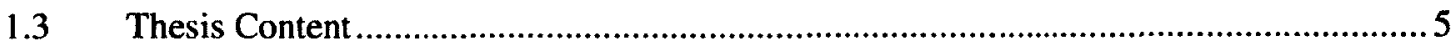

2 Chapter: Literature Review.......................................................................................... 7

$2.1 \quad$ Conceptual Model .................................................................................................

2.2 Requirements Engineering and Social Modeling ………............................................ 8

2.2.1 Requirements Engineering …………………..............................................

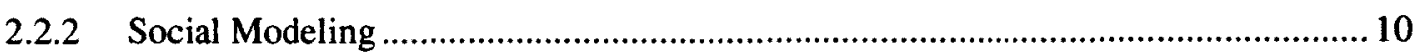

2.3 Current Models for early analysis ............................................................................ 11

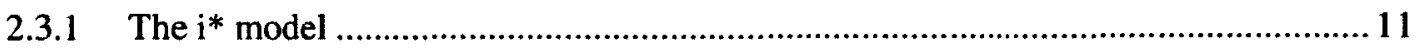

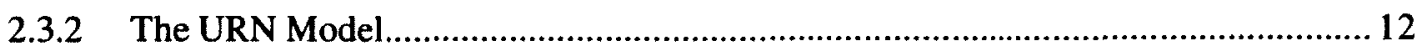

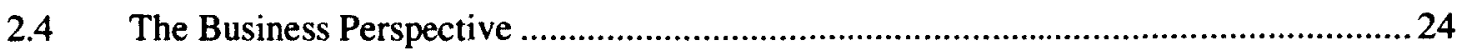

2.4.1 Business Process ..............................................................................................2

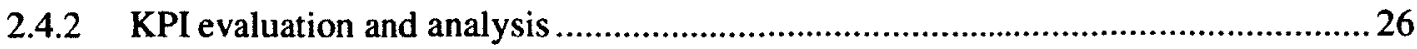

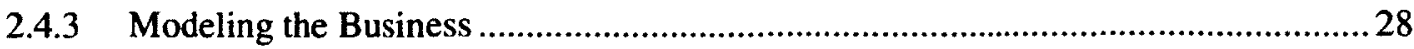

2.5 Applications of $\mathrm{i} *$ and URN models to different domains ...........................................29 
2.5.1 Modeling with $\mathrm{i}^{*}$ .29

2.5.2 Applications of the URN model to different domains ....................................... 30

3 Chapter: Working System Model................................................................... 32

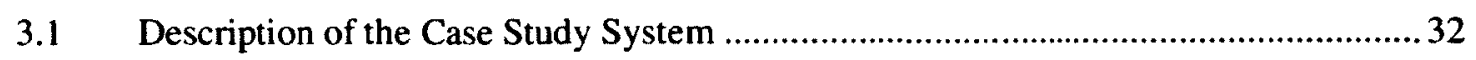

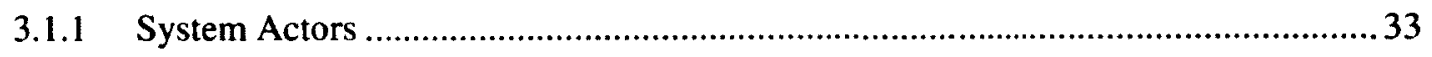

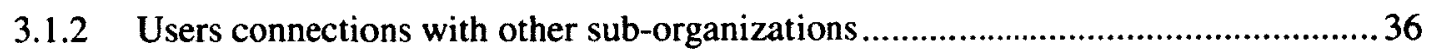

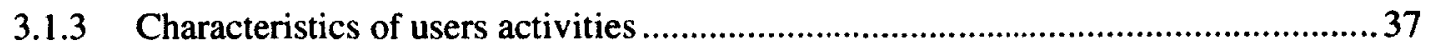

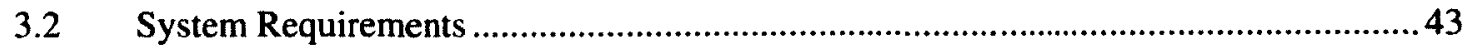

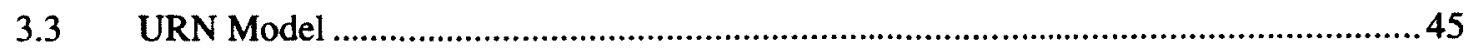

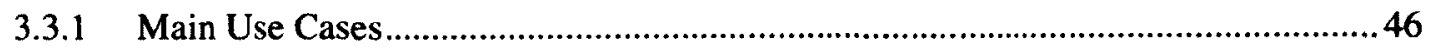

3.3.2 A look at the working system's options.........................................................47

4 Proposed System Model ................................................................................ 83

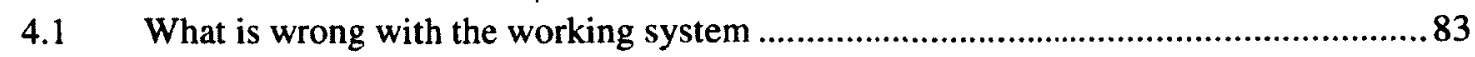

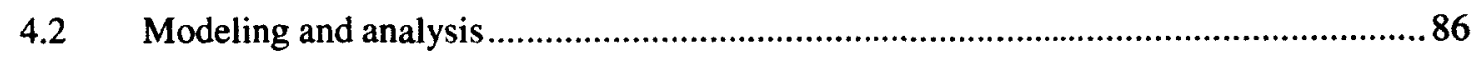

5 Chapter: Performance Evaluation ................................................................ 101

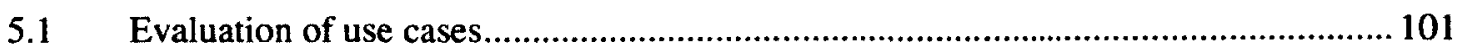

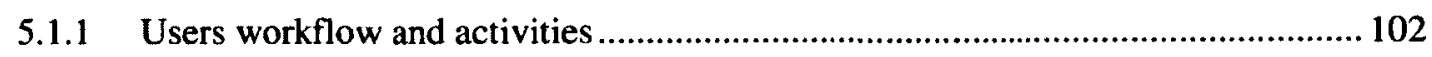

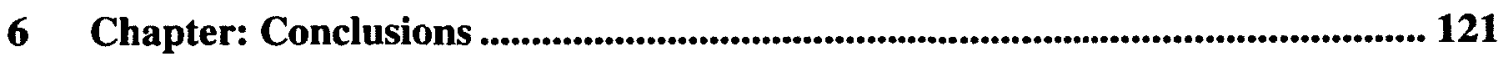

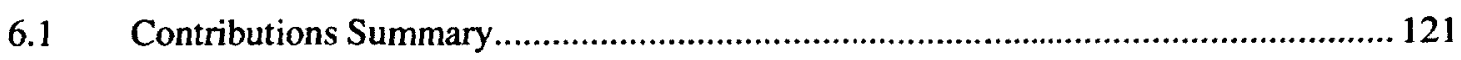

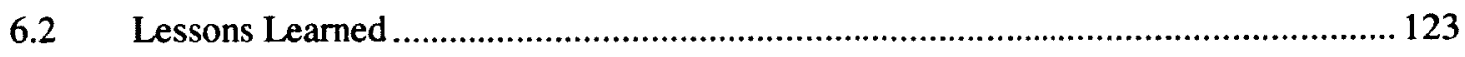

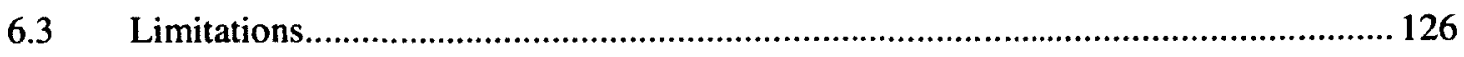

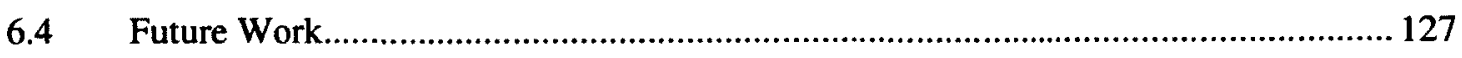

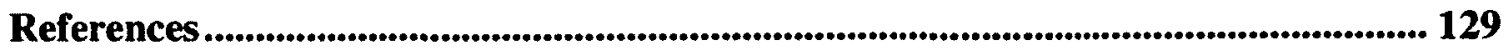

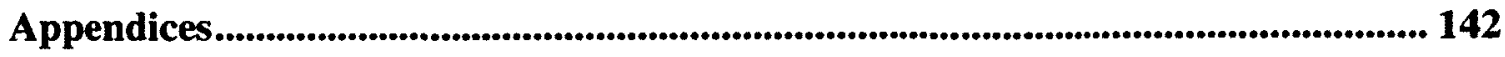




\section{List of Tables}

Table 2.1: Problems generated by requirements ([European96, Lutz93, Standish95]) .... 10

Table 2.2: URN Characteristics ([Amyot02a, Amyot03a, Amyot04, ITU08]) .............. 13

Table 2.3: Characteristics of GRL ([Amyot03b, Saleh02]) ...................................... 14

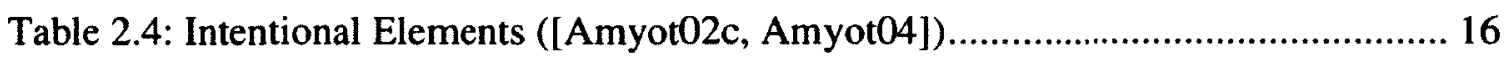

Table 2.5: Intentional Relations, connecting elements ([Amyot02c, Amyot04])............ 16

Table 2.6: UCM Characteristics [Amyot99b, Amyot01a, Amyot0lb, deBruin01, Liu01a,

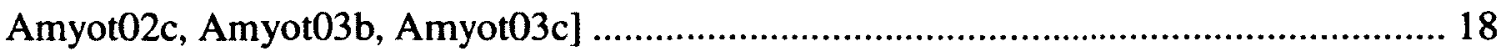

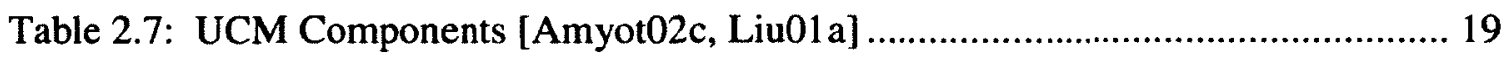

Table 2.8: Contribution of tools to realization of goals taken from [Roy07] ................. 22

Table 2.9: Applications of the $i^{*}$ modeling tool [Amyot03a, Kethers05, Maiden04] ..... 29

Table 2.10: Applications of the URN modeling tool [Amyot05a,

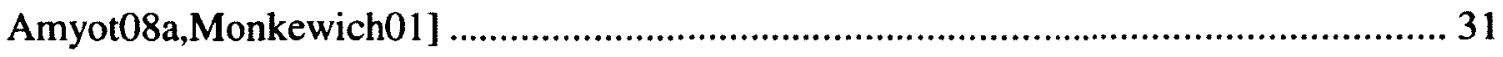

Table 5.1: Presentation of Intentional Elements per each Actor ............................... 104

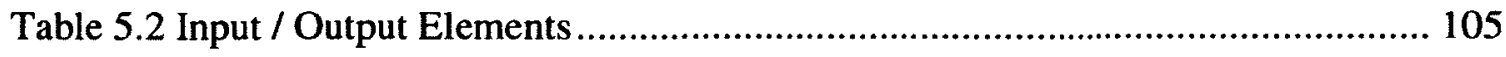

Table 5.3: Evaluation of main Users goals, working system ................................... 113

Table.5.4: Evaluation of goals and Actors, working system ................................. 113

Table 5.5: KPI Evaluation of Users goals, working system ................................... 116

Table 5.6: KPI Evaluation of goals and actors, working system ........................... 116

Table 5.7: KPI evaluation for Mean Time and Ability, working system ..................... 117

Table 5.8: KPI evaluation for Keep Cost Low and Customer Satisfaction, working system 
Table 5.9: Evaluation of main Users goals (proposed system) .............................. 119

Table 5.10: KPI Evaluation of main Users goals............................................... 119

Table 5.11: KPI Evaluation of Users Management and Actors................................. 119

Table 5.12: Evaluation of KPI 01 for Users main ................................................ 119

Table 5.13: KPI Evaluation of Low Cost' and Customer Satisfaction .......................... 119 


\section{List of Figures}

Figure 1.1: Cost to resolve errors [McConnell93] ................................................. 4

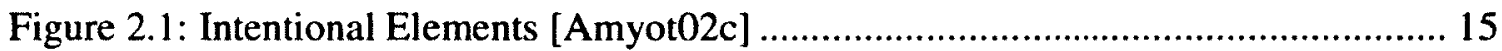

Figure 2.2: Representation Intentional Elements................................................... 15

Figure 2.3: Representation of Contributions [Amyot08b] .................................. 16

Figure 2.4: Representation of Actor ITManagement ............................................ 16

Figure 2.5: Extras from GRL Graph showing Actors, Intentional elements and relations 17

Figure 2.6: UCM components, stubs [Amyot09]................................................... 19

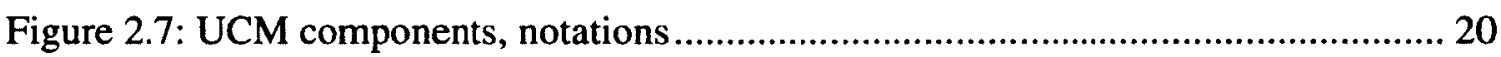

Figure 2.8: - Extras from UCM diagram, depicting UCM components ...................... 20

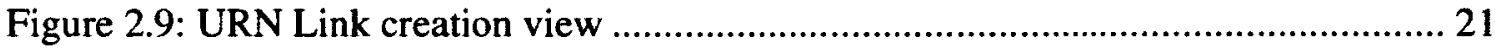

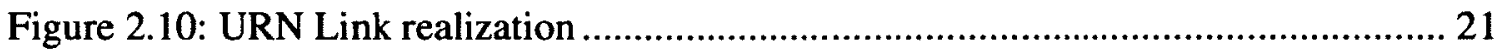

Figure 2.11: Example of URNLinks [jUCMNav12] ........................................ 21

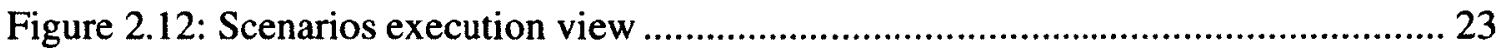

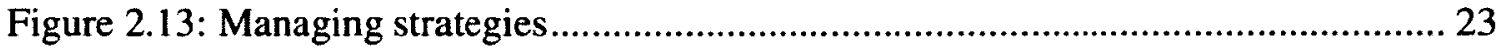

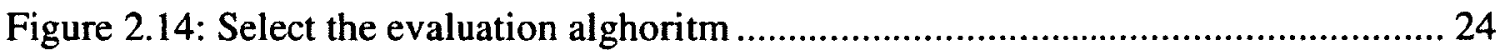

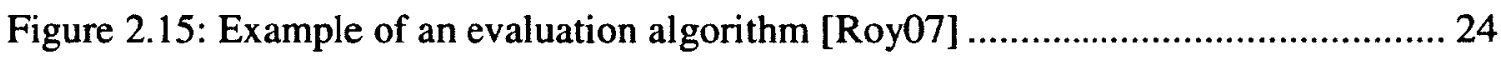

Figure 2.16: Example of evaluation results of Intentional elements .......................... 24

Figure 2.17:Example of evaluation results for an Actor Management .......................... 24

Figure 2.18: Example of Business Process Management (BPM) Service Pattern [NIH07] 
Figure 3.1: Example High-level view of Organization (n, a positive integer at least

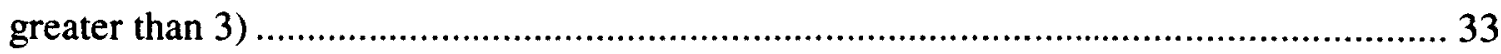

Figure 3.2: Example Presentation of services/activities managed by ITD and BD........ 36 Figure 3.3: High level view of the system, presentig the Actors with few high-level goals 48

Figure 3.4: Detail representation of a GRL Graph .............................................. 49

Figure 3.5: Summary of scenarios for User activities and work on the system.............. 52

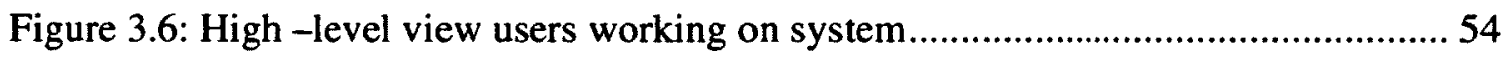

Figure 3.7: Example Scenario (1) Prepare Deployment (Stub 3.6.1) ............................ 54

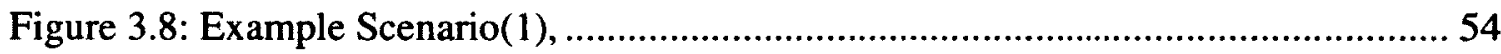

Figure 3.9: Example from Scenario (1) IdentifyIssues (ReportProblems) (Stub 3.6.4) ... 54

Figure 3.10: Example from Scenario (1) IdentifyIssues (ReportProblems) (Stub 3.6.2) 55

Figure 3.11: Extras from an MSC (some lifelines may not be shown)......................... 56

Figure 3.12: Example of URN Links ............................................................... 56

Figure 3.13: Administrative information propagation across the system ....................... 58

Figure 3.14 - Information propagation scenario............................................ 59

Figure 3.15: Overview of information propagation through the system (Users view) .... 60

Figure 3.16: Extract from detailed look into the reporting information use case ............ 61

Figure 3.17: Extract from detailed look into the reporting information use case ........... 62

Figure 3.18: Detailed view of the complex work of the users (Stub 3.17.1) ..................63

Figure 3.19: Detailed view - CARs/issues handled (Stub 3.17.2)........................... 63

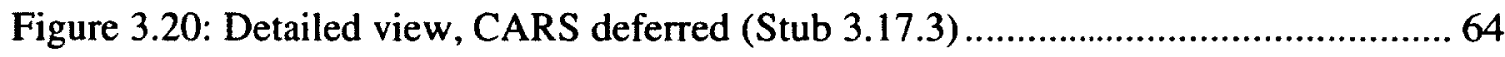

Figure 3.21: Defer to project low priority development requests (Stub 3.17.4) ............. 64 
Figure 3.22: Defer to project low priority intranet issues (Stub 3.17.5) .........................64

Figure 3.23: GRL graph presenting details for test and deploy case ......................... 70

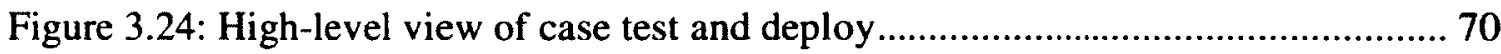

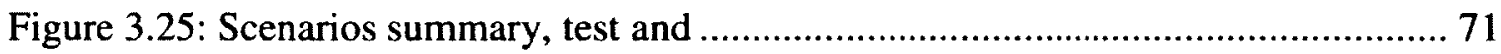

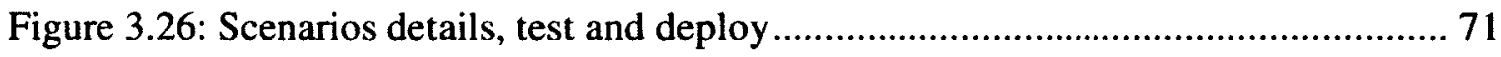

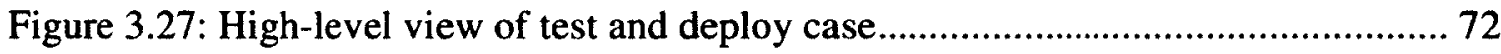

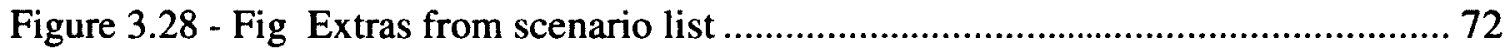

Figure 3.29: Sub-map business IT develops (Figure 3.27, Stub 3.27.1) ..................... 73

Figure 3.30: Overview of ReceiveRequest (Stub 3.29.1) ...................................... 73

Figure 3.31: Details on Request ApprovalOn Implementation (plug-in for Stub 3.29.2) 74

Figure 3.32 - Details on ProvideTraining Stub (3.29.3) ............................................. 74

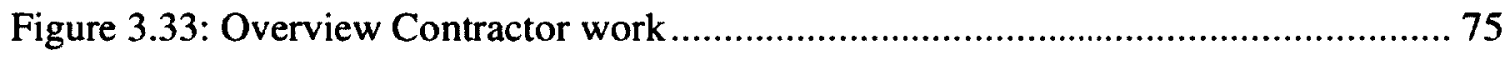

Figure 3.34: Scenarios list Contractor ........................................................... 75

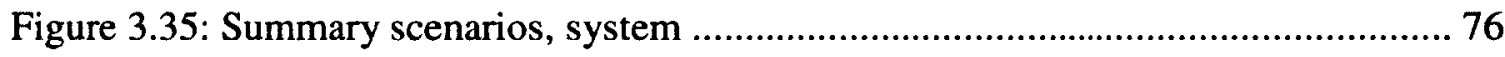

Figure 3.36: Overview of users maintenance work .................................................... 76

Figure 3.37: Users maintenance work, sub-map Stub (3.36.1)................................ 76

Figure 3.38: User work, request (Stub 3.36.2) implementation approval...................... 77

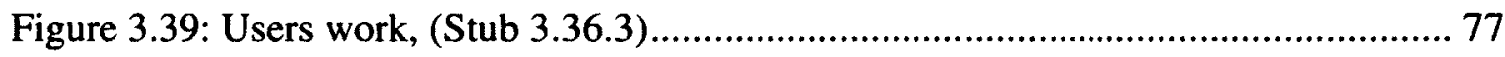

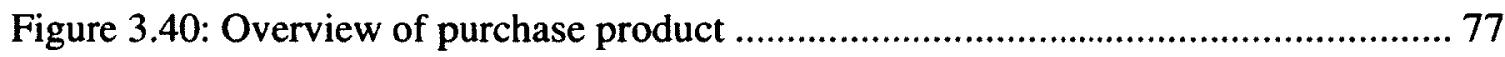

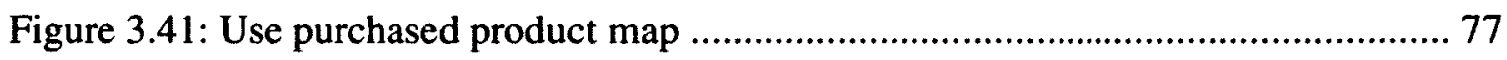

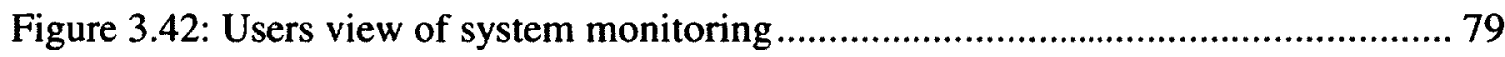

Figure 3.43: Detailed graph of Users view on monitoring .................................. 79

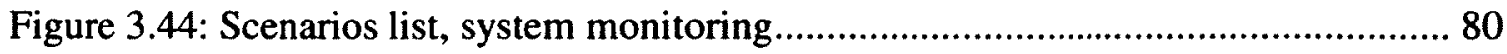


Figure 3.45: High-level map, system monitoring ………………………................... 80

Figure 3.46: View of RequestBusinessApproval (Stub 3.45.1) f..................................... 80

Figure 3.47: Contact Business Stub (3.45.2) …………............................................... 81

Figure 3.48: Monitoring Options (Stub 3.45.3) …………............................................ 81

Figure 3.49: IT Monitors Users system (Stub 3.45.3) ................................................ 82

Figure 4.1: High -level diagram presenting in details BD and ITD services .................. 87

Figure 4.2: Example of information propagation in the system .....................................8 88

Figure 4.3: Comparing the GRL graphs for the two systems ......................................... 90

Figure 4.4: Reporting issues: comparing the two models............................................ 91

Figure 4.5: Asses issues: comparing the two systems ................................................. 91

Figure 4.6: Compare deployment process on the two systems........................................ 92

Figure 4.7: Compare administrative information propagation on detailed GRLs ........... 94

Figure 4.8: Compare administrative information propagation UCMs in both systems.... 95

Figure 4.9: Compare handling of issues maps for both systems .................................... 95

Figure 4.10: Compare test and deploy high-level view for both systems......................... 96

Figure 4.11: Compare test and deploy detailed graph for both system ............................ 96

Figure 4.12: Compare high-level test and deploy maps ................................................ 97

Figure 4.13: Request for approval process in the working system .................................. 98

Figure 4.14: Request for approval process in the proposed system................................. 98

Figure 4.15: Compare system monitoring high-level graph in the two systems .............. 99

Figure 4.16: Compare detailed system monitoring graph for both systems ..................... 99

Figure 4.17: Compare Users monitoring capabilities on both systems ......................... 100

Figure 5.1: Evaluation of Strategy (1.1) ................................................................ 107 
Figure 5.2: Evaluation of Strategy (1.2) ……………........................................ 107

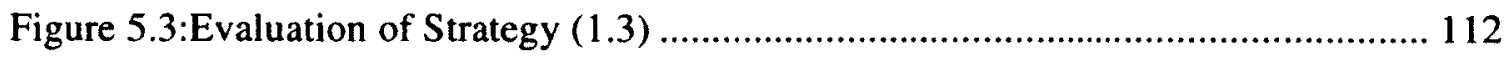

Figure 5.4:Evaluation of Strategy (1.4) ............................................................. 112

Figure 5.5: Evaluation of Strategy (1.5) ............................................................... 113

Figure 5.6: KPI strategies/working system ................................................................. 115

Figure5.7: KPI Strategies, extract from pdf report .................................................... 115

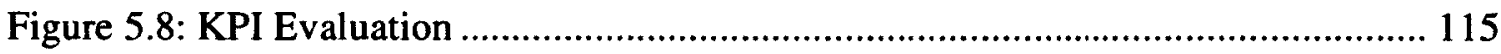

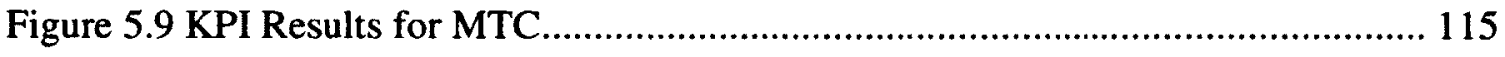

Figure 5.10 KPI Results for Availability ............................................................. 115

Figure 5.11: Example of satisfied performance for KPI MTC Strategy (2.3) ................ 116

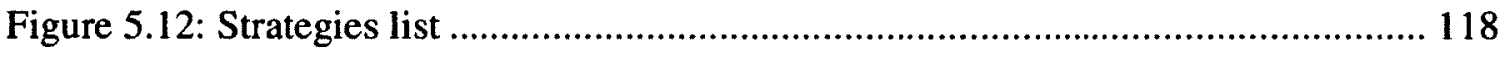

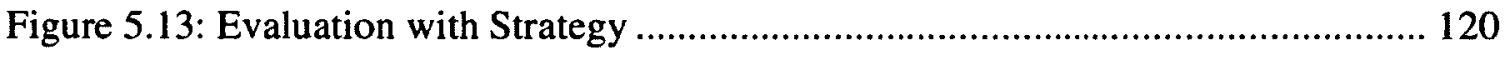

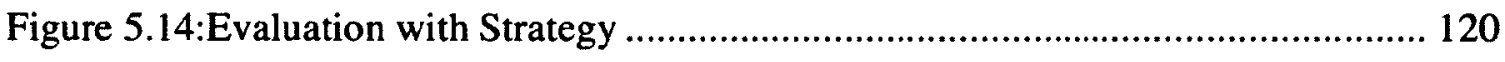

Figure 5.15: Example of KPI Results for Strategy 2.1 …........................................ 120 


\section{List of Appendices}

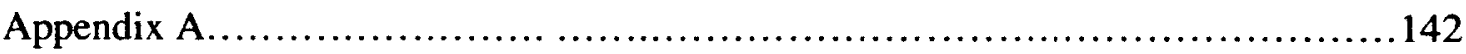

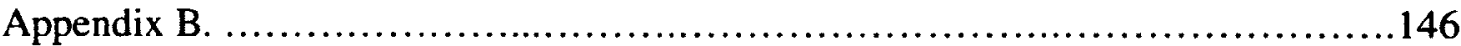

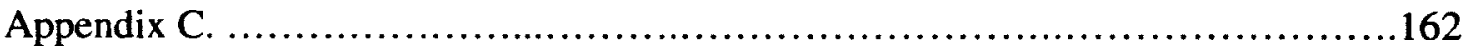




\section{List of Acronyms}

$\begin{array}{ll}\text { Acronym } & \text { Acronym Definition } \\ \text { BD } & \text { Business Department } \\ \text { BDM } & \text { Business Department Management } \\ \text { BPM } & \text { Business Process Management } \\ \text { CAR } & \text { Corrective Action Request } \\ \text { CM } & \text { Conceptual Models } \\ \text { FR } & \text { Functional Requirements } \\ \text { GRL } & \text { Goal-oriented Requirement Language } \\ \text { ITD } & \text { Internet Technology Department } \\ \text { ITM } & \text { Internet Technology Management } \\ \text { ITDNG } & \text { Internet Technology Department Network Group } \\ \text { ITDSG } & \text { Internet Technology Department Solution Group } \\ \text { jUCMNav } & \text { Eclipse plug-in, developed to support the ITU-T's URN Z.151 } \\ \text { KPI } & \text { Key Performance Indicators } \\ \text { LD } & \text { Legal Department } \\ \text { NC } & \text { Network Component } \\ \text { N-FR } & \text { Non-Functional Requirements } \\ \text { RD } & \text { Regulatory Department } \\ \text { RE } & \text { Requirements Engineering } \\ \text { SADT } & \text { Structured Analysis and Design Technique } \\ \text { SC } & \text { Specific Component } \\ \text { SO } & \text { Sub-organization, department of Organization } \\ \text { UCD } & \text { Use Case Diagrams } \\ \text { UCM } & \text { Use Case Map } \\ \text { UML } & \text { in the Unified Language Modeling } \\ \text { URN } & \end{array}$




\section{Chapter: Introduction}

\subsection{Objectives and Motivation}

The objectives of the thesis consists in modeling, understanding and evaluating the operation, maintenance, refurbishment and evolution of very complex distributed systems in early development phases. The kind of system considered here is a component of a complex distributed system that serves a large scale country-wide organization.

This organization is composed of many modules or sub-organizations which have distinct operational mandates and their own managers, receive distinct budgets and operate with their own staff members. Often different sub-organizations (including users, stakeholders, legal, regulatory, business analysis and internet technology departments, which have different responsibilities, access rights and goals) are involved in inter-departmental projects across the enterprise and in external projects with various clients, having their work based on contracts, memoranda of understanding or legal agreements established between the participants. The roles of the users from our case study are related to mandatory activities; they include receiving tens of thousands of data records on annual basis from various clients that are interested in what the system does and contribute to the system's requirements; responding to thousands of requests received from external clients, which include companies, government agencies, universities, the Canadian public; providing data analysis reports on annual basis for the use of the public, as well as ad-hoc reports requested by some clients, including regulatory departments, the organization's stakeholders and universities.

The modeling language used in the thesis is the User Requirements Notation (URN) [Amyot04] which is supported by the jUCMNav tool [JUCMNav a]. URN is a modeling 
language that permits system engineers to model the requirements of an existing system or of a system to be developed and to ensure that they are complete and correct. The URN is composed of two views that complement each other: the Use Case Map (UCM) and the Goal-oriented Requirement Language (GRL) notations. URN enables the combination of different scenarios for operational aspects and rationalization of structural and performance means represented in UCM, with modeling notations and abstractions for intentions and planned objectives supported by GRL. URN is normally used for requirement identification, specification, analysis and validation, including the functional and non-functional requirements of the system. The URN notation is supported by the jUCMNav tool, which is a free plug-in for Eclipse, providing the graphical editing environment and the transformation and analysis means that support the URN.

The focus of the thesis is on a subset of the system requirements related to the way in which the operation of the system is organized, to the interaction between different departments involved in the system activities and the impact of this interaction on the realization of different goals. We are concerned with how the information is transmitted between different operational modules across the organization, what type of processes and reporting loops have been implemented. We intend to understand how the administrative information is being monitored at system level and how system matters are being reported to system administration, management, users and clients; to determine who will address and initiate corrective actions requests and how they will propagate across the organization; and to identify who needs to know what for a distributed system model that has different managers for each component module. The model is based on an existing real system, which is called from here on the working system model. During the 
construction of the model, an analysis and evaluation was done. This has allowed us to understand how different goals are achieved for different organization's components; to determine strategies that are less supportive for the realization of objectives; to identify goals that could be achieved or not, at the planned satisfaction level, for specific strategies. As a result, we realized there is room for improvement. A new version of the system, called proposed system is described and compared with the working system.

In most of the cases, the models have strategies and scenarios ending with different results. Usually scenarios have both positive consequences and negative effects, depending on the components characteristics, relationships contributions and what specific resolution has been applied. The outcome of scenarios really depends on how much the sub-organizations involved are in charge, comprehend, and carry on the management of requirements. According to studies [Easterbrook04], these can be reflected by the following indicators: a) the expense for resolving system issues, b) the reasons for unsuccessful projects and c) the causes for success. Studies that have looked at the life cycle of projects [Boehm81], [Lamsweerde00], starting with the requirements phase and ending with the deployment of the final product, have demonstrated that the successful completion of a project or system implementation is tightly linked to the requirements. They have estimated that it could be almost 200 times more expensive to correct requirements [Boehm81] and fix the errors generated at a later date, compared to modeling, analyzing, evaluating the problems at an earlier date, when the requirements were gathered. Results of this survey [Easterbrook04] are indicating the factors that could lead to successful system implementations or to cause unsuccessful projects. The factors with positive contributions are user's participation, engagement and sustainability from 
management, and clear and complete requirements [Kotonya98]; while negative impacts are generated by lack of interest in the user's participation and contribution, difficulties

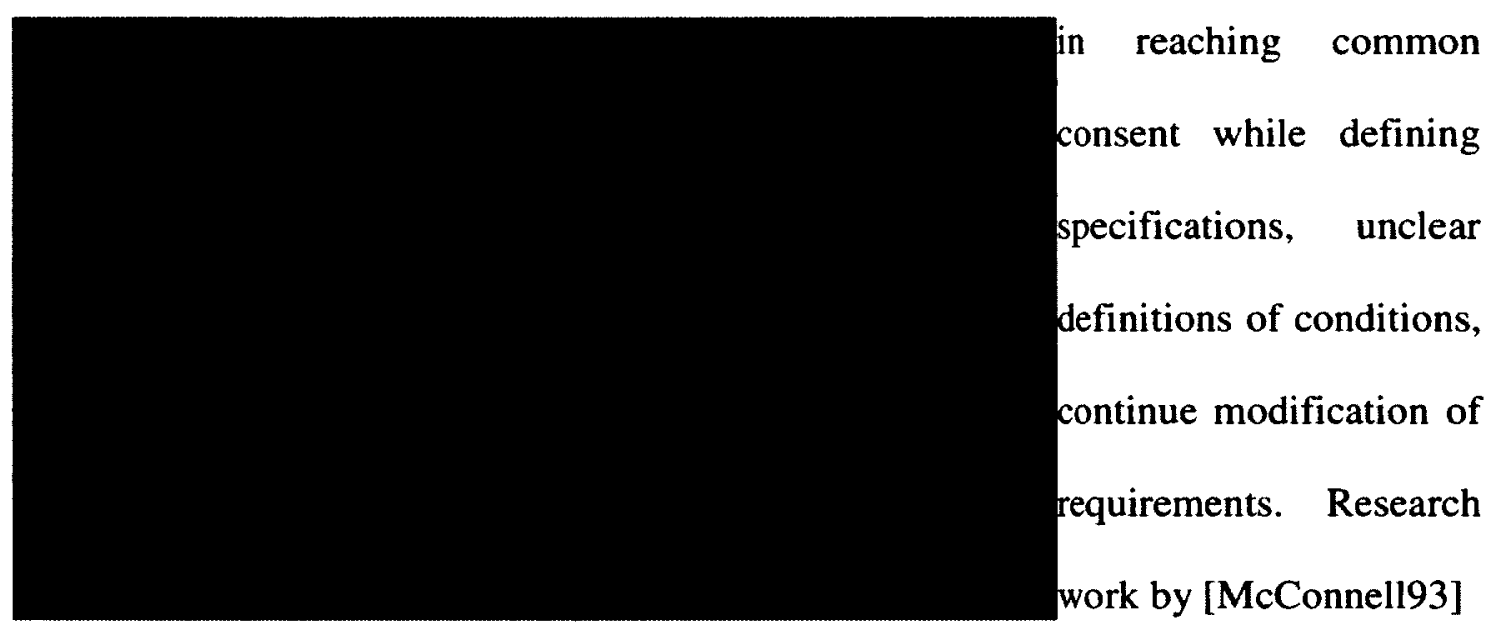

Figure 1.1: Cost to resolve errors [McConnell93]

has demonstrated that "the cost of incorrect or incomplete requirements is extremely high" as per (Figure 1.1) which makes more difficult and expensive to correct errors in any development phases except analysis. We have modeled the working system to be able to understand how it operates, what could cause functional/operational issues, and to learn how the problems could be improved.

\subsection{Thesis contributions}

The contributions of the thesis are as follows:

1. Develop a URN model of a complex system, inspired from reality, with participants from different departments across a very large organization, which have different goals and responsibilities and operate with their own budget, managers and staff.

2. Use the URN model to understand and evaluate how the goals of different actors can be achieved in our case study system. The objectives were as follows: understand 
how the administrative information is being monitored at system level; analyze monitoring techniques; report system issues to stakeholders, to system administration and to users; determine who will address and initiate the corrective actions, how the corrections will propagate and what will be the impact at different system levels.

3. Design the URN model of a new system by transferring some responsibilities managed at organizational level by other departments to the sub-organization that uses and owns the system. The objective is to reduce the overhead due to the backand-forth reporting of administrative information to other sub-organizations that have different goals and responsibilities, and different managers; to reduce the delay in addressing and resolving requests collected at an organizational level from all suborganizations; to increase the system knowledge and to improve the monitoring of the system with services that will ensure continuous assistance 24 hours a day.

\subsection{Thesis Content}

The material of the thesis is structured as follows.

Chapter 2 describes the literature review. We present background information on engineering techniques used to gather system requirements, including: conceptual models, requirements engineering and social modeling. We provide a review of current modeling notations, such as $i^{*}$ star and URN, and a summary of their applications in other domains than engineering and telecommunications.

Chapter 3 presents the URN model of a working system inspired from reality. During the modeling process, our focus was on a subset of the system requirements which are concerned with the way the operation of the system is organized, how different 
departments involved in the system activities interact and what is the impact on the realization of the goals, how is the information transmitted between different operational modules across the organization, what type of processes and reporting loops have been implemented; understand how the administrative information is being monitored at system level and learn how system matters are being reported to system administration, management, users and clients; determine who will address and initiate corrective actions requests and how they will propagate across the organization; identify who needs to know what for a distributed system model that has different managers on component modules.

Chapter 4 introduces the proposed new system. During the modeling of the working system, its evaluation was done and we realized that the system has various inefficiencies. A new version, called proposed system is described in this chapter and its URN model is presented.

Chapter 5 compares the results of the evaluation of the two models, the working and the proposed system. The strategies used for the evaluation of the goal satisfaction levels are described, key-performance indicators are defined and used for evaluation. The results of the two models are compared step-by-step and the causes for the difference in results are analyzed.

Chapter 6 summarizes the work on the thesis by presenting our contribution and discussing benefits and conclusions, and identifies directions for future work. 


\section{Chapter: Literature Review}

In this chapter we present background information on engineering techniques used to gather and analyze system requirements. The following sections provide a review of the current modeling notations and the supporting tools.

\subsection{Conceptual Model}

As presented in [Mylopoulos04], Conceptual Models (CM) also known as "visual models" are used to "describe proposed requirements and design" characteristics "for the new system"; they provide the tools "to capture people's understanding (conceptualization) of what is being modeled, and are usually represented in terms of a graph structure". Studies such as in [Mylopoulos04] are showing that the basis of CM have been set around the 60s and 70s, starting with "semantic networks" [Quillian63, Quillian68], followed by SIMULA [Dahl66], the programming language developed to describe the events of a system. Next were developed the "semantic model" [Abrial74] and the "entity-relationship model" [Chen76]. They evolved rapidly into "Structured Analysis and Design Technique (SADT)" [Ross77b] created to share believes, interpretations and views, and for specifying requirements. CMs are independent of implementation issues and design concerns. An instance of $\mathrm{CM}$ is presented in [Embley11] as code that communicates with the machines, providing information to be executed. Related studies [Yu01a, Yu01b] are reporting an increased interest in using CMs to design systems, and are recognizing the essential role [Lamsweerde00] of the requirements engineering in the design of the product to be built. The $\mathrm{CM}$ based on Requirements Engineering (RE) approach is a method to refine the "quality of the 
software production process" [Insfran02]. The method uses a complex mechanism to identify functional attributes of the system and to break down high level requirements into accurate qualifications able to describe the architecture of the system.

\subsection{Requirements Engineering and Social Modeling}

In the last 10 years it has been acknowledged that requirements are a source of concern for software development. Even early studies have raised awareness that "inadequate, inconsistent, incomplete or ambiguous requirements" could have "a critical impact on the quality" [Bell76] of the final product. Various lessons learned explained that "the requirements for a system do not arise naturally; they need to be engineered and have continuing review and revision" [Bell76]. They must define the needs of the system [Ross77a], as the main components in the process of gathering requirements [Kotonya98] for applications in software and engineering domains. Studies on projects life cycles [Boehm81, Lamsweerde00], starting with the requirements phase and ending with the delivery of the fine product, have concluded that the successful completion of a project or implementation of a system is tight to its requirements, and have estimated that it could be almost 200 times more expensive to correct requirements and fix the errors generated at a later date rather than at the time when the requirements were gathered.

\subsubsection{Requirements Engineering}

Requirements depict the goals of a software intensive system [Easterbrook04], being engaged with system concerns, functionalities, attributes and relationships created between them and their qualifications [Zave97]. They provide the foundation for understanding how the system works and what it supposed to do [Roy07], enabling us the 
notations and tools to assess if the system design is good and how well it will meet the expectations if specific design configurations were chosen [Easterbrook04, Roy07]. The requirements contain two categories: Functional Requirements (FR - the specifications that indicate what the system should do), and the Non-Functional Requirements (N-FR the specifications that indicate the attributes and restrictions of the system). Different methods have been developed to handle RE [Roy07] including requirements elicitation, analysis, specification, verification and management [Boldt01, Nuseibeh00]. Even though software RE methods were created for structural programming to capture declarative, behavioral and interactive aspects of systems, goal-oriented requirements analysis has been proposed more recently [Mylopoulos99, Roy07]. To support this analysis type, new concepts have been developed [Yu97, Yu01c]. They have enabled the capability of investigating possible choices, resolutions and adjustments, instead of looking only for FR [Yu98]. The goal-based interpretation is important [Lanwerde00] for investigating software limitations, management disagreements, handling of exceptions and administration of design options. In parallel, scenario modeling approaches [Amyot03d] that are easy to follow by the stakeholders, were created to present requirements [Roy07] and to help identify hidden issues.

\section{Challenges in RE}

Studies [Som97] and work experience in the domain have reported a type of pressure and a demand in the competitive industry for "shorter software development cycles [Som97]. Different development projects and systems refurbishments have been limited due to environment and IT policies, being forced to fit in a given system architecture. Concerns have been raised [Som97, Sawyer01] on this approach, advising that "is almost always 
impractical to implement requirements engineering as a linear, deterministic process where system requirements are elicited from the stakeholders, baselined, allocated, handed over to the software development team". Such circumstances could have helped in identifying problems, later in the process, with the price of an expensive correction work. In other cases described in [Lutz93, Standish95, European96, Nuseibeh00] and presented in (Table2.1), systems have been delivered without meeting the requirements due to weak RE. Reports on these cases concluded that unfinished specifications and requirements [Standish95] were ranked second of the most important reasons that lead projects to failures.

Table 2.1: Problems generated by requirements ([European96, Lutz93, Standish95])

\begin{tabular}{|c|c|c|c|}
\hline \multicolumn{2}{|c|}{ stidr hopic } & 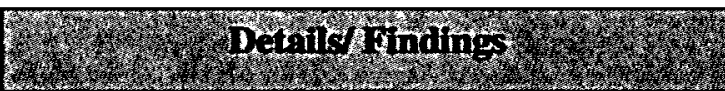 & 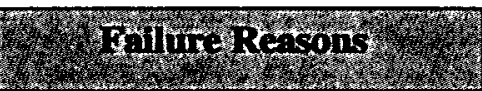 \\
\hline 1 & $\begin{array}{l}\text { Study of } \\
\text { errors }\end{array}$ & $\begin{array}{l}\text { Studies of errors found in NASA Programs. } \\
\text { Safety issues found in different software } \\
\text { applications [Lutz93] }\end{array}$ & $\begin{array}{l}\text { Errors found in FR and in } \\
\text { interface requirements in , } \\
\text { Galileo, Voyager projects }\end{array}$ \\
\hline 2 & $\begin{array}{l}\text { Survey } \\
\text { conducted } \\
\text { in USA }\end{array}$ & $\begin{array}{l}\text { From } 8000 \text { projects identified as started in } \\
350 \text { companies, only half were completed. } \\
\text { Out of all projects, the ones not finalized } \\
\text { were } 1 / 3^{\text {rd }} \text {, partial completed were } 1 / 2 \text {. The } \\
\text { rest were partial completed with delays, over } \\
\text { budget and with missing components }\end{array}$ & $\begin{array}{l}\text { Incomplete requirements, } \\
\text { lack of users input and } \\
\text { participation, constant } \\
\text { changing of requirements, } \\
\text { "unrealistic expectations and } \\
\text { unclear objectives" [ Sta95] }\end{array}$ \\
\hline 3 & $\begin{array}{l}\text { Survey } \\
\text { conducted } \\
\text { in Europe }\end{array}$ & $\begin{array}{l}\text { For approximate } 3800 \text { companies located } \\
\text { across } 17 \text { countries had problems related to } \\
\text { software applications. }\end{array}$ & $\begin{array}{l}\text { "Requirements specification } \\
\text { and management" [ESI96] }\end{array}$ \\
\hline
\end{tabular}

\subsubsection{Social Modeling}

With the rapid development of technology, new challenges appeared, stressing out on the boundaries of modeling and indicating interest in modeling not only the system, but also its environment [Yu09]. New issues have also emerged, questioning if the system would be able to deliver each planned application and support the organization in achieving its objectives; if it would be beneficial to be used in specific circumstances, and if there were 
any available alternative options [Santader02]. In [Mylopoulos98a] it has been indicated that modeling was growing stronger, considering the importance of business characteristics in the organization's life, and that it was the right time for new types of modeling, intentional and social, to respond "to the emerging needs of the information revolution" [Mylopoulos98a]. Recent studies [Yu09] have shown that social modeling [Kling96, Yu09] has the methods and the tools to enhance software development

\subsection{Current Models for early analysis}

For many years, models have been developed representing two views: the static (structural view) through class diagrams and the dynamic (behavioral view) through state charts and/or interaction diagrams [Yu09], having the FR identified with Use Case Diagrams (UCD) in the Unified Language Modeling (UML) [Santader02]. Given the limitations of UML "for modeling organizational requirements" [Santader02], new tools have been developed. Techniques like $\mathrm{i}^{*}$ and User Requirements Notation (URN) have been created to support the modeling of organizational aspects [Amyot03e, ITU08]. The $i^{*}$ model works with goals to characterize actor's profiles, to enable the modeling of management concerns, responsibilities [Roy07]. URN integrates FR/N-FR [Amyot03a, Amyot02c] supporting identification, presentation, specifications analysis, enabling links between business processes and goals [Pengfei07].

\subsubsection{The i* model}

The $i^{*}$ model has been designed to be used in the early stage of RE [Roy07] and has been presented for the first time in 1993 at the "International Symposium on Requirements Engineering" [Yul1]. Its development included the intention of integrating social aspects 
in system modeling, ensuring a leading role of the social characteristics in the development phase. Intentional aspects are collected when requirements are gathered, describing what is desired by the actors, how they plan to achieve goals and who is involved in relationships. There are slight differences between the $\mathrm{i}^{*}$ and other modeling tools. The $\mathrm{i}^{*}$ modeling is supported by "intentional relationships amongst agents, called actors" [Roy07, Yu01c]. They rely on each other to fulfill their "intentional elements" [Roy07] and are related to tasks to be completed, goals to be accomplished and resources to be assigned [Yu97, Yu01, Yu09]. UML also works with goals, of functional type, and actors that participate in the completion of operations, while the KAOS model [Yu09] accepts all types of goals without being concerned about the actor's intentions. New tools have been developed to support $\mathrm{i}^{*}$ and the modeling of complex systems. Some are open sources and work with Eclipse [jUCMNava, J-PRiM, OpenOME], while others have been developed "on the programmability of general purpose tools" [Lockerbie06]. With the diversity of meta-models it has been proposed to build an adequate exchange format [Cares08], to resolve different characteristics [Lucena08] and manage the issues created by many versions of a certain model [Sabetzadeh06]. In $2008, \mathrm{i}^{*}$ has been included in standard ITU-T Z.151 [ITU08, Liu01a].

\subsubsection{The URN Model}

As presented in [Amyot99a] and ITU-T Z.151 recommendation [ITU08], the URN has been "intended for the elicitation, analysis, specification, and validation of requirements" [Amyot08a]. The acceptance of URN as a standardized notation in 2008 [Amyot11a, ITU08, Liu01] brought the recognition of RE activities, proving that they are accurate, reliable and able to be predicted, while producing correct, complete and consistent 
results. URN has two components [Amyot02c, Amyot03a] that complement each other: Goal-oriented Requirement Language (GRL) and Use Case Maps (UCM). GRL describes FR and why type of questions, and UCM describes N-FR [Roy07] and what type of questions [Bochmann 10].

Table 2.2: URN Characteristics ([Amyot02a, Amyot03a, Amyot04, ITU08])

\begin{tabular}{|c|l|}
\hline 1 & Supports the development of the system and performance analysis in early stages \\
\hline 2 & Facilitates the observation of requirements and understanding of their interactions; \\
\hline 3 & Allows to change requirements during their development \\
\hline 4 & Enables the transition from user requirements to FR and N-FR \\
\hline 5 & Presents scenarios with no need to refer to subcomponents of the system \\
\hline 6 & $\begin{array}{l}\text { Facilitates the transition ofrequirement's characteristics to a high level schema, allowing } \\
\text { identification, consideration of requirements that are pending management's approval }\end{array}$ \\
\hline 7 & Enables identification and preveting of unwanted connections between services \\
\hline 8 & Supports reusable components, analysis and goal's rules, architecture design options \\
\hline 9 & Supports the transformation of requirements in UML,MSC [ITU08] \\
\hline 10 & Enables the connection of URN components to external entities \\
\hline 11 & Provides support in early system development, facilitating of stakeholders participation \\
\hline
\end{tabular}

The characteristics of RE, presented in Table 2.2 above, have been captured in the URN by joining goals with scenarios in a successful combination [Bochmann 10] that enabled in URN the capability to have a smooth transition [Sakkina03] from analysis to design modeling [Amyot03b]. The characteristics have been improved and included in standard documentations [Saleh04]. Through modeling we can explain how applications and systems work, having the capability to analyze and evaluate if expected results could be 
achieved. A picture "it's worth a thousand words" [Syracuse911], and the graphical presentation will contribute to the understanding of technical details for the users without a technical background. With URN we can model requirements specifications for current products or additions; the process, from presenting the goals to finding alternatives and improving processes, creating a view of the system in an intuitive graphical notation; we can discover the interactions between sub-organizations involved in the development/maintenance of the system, including management, high-level stakeholders; and identify potential vulnerabilities and "weaknesses in existing relationships" [Yu09]. The GRL component. GRL is a graphical notation [Amyot02c, Amyot03a] that models N-FR, including performance, cost, security and usability [Chung00], considerations, alternative solutions for reaching objectives, business goals [Roy07] and management concerns [Pengfei07]. They enable the presentation of actions, hypothesis, alternatives, available options, believes, rationales, relationships, as presented in (Table 2.3).

Table 2.3: Characteristics of GRL ([Amyot03b, Saleh02])

\begin{tabular}{|c|l|}
\hline 1 & Supports identification of user's requirements, even if development data is very little \\
\hline 2 & Has the ability to examine the relationships and links between requirements and goals \\
\hline 3 & Presents the sequence of events, without requiring communication between components \\
\hline 4 & Monitors the tranzition from specifications to a high-level composition \\
\hline 5 & Introduces in the works of software engineering a schema to help taking decisions \\
\hline 6 & Recognizes other types of architectures and the estimation of new requirements \\
\hline 7 & \begin{tabular}{l} 
Provides the tool to document decisions; it can be used repetitevely and separately \\
\hline 8
\end{tabular} $\begin{array}{l}\text { Is an agent oriented based modeling notation, with reasoning capacity on requirements: } \\
\text { user interface, performance, usage, security settings, social context, legal demands }\end{array}$ \\
\hline 9 & $\begin{array}{l}\text { Supports the ability [Amyot03b] of assigning responsibilities from scenarios to design } \\
\text { components }\end{array}$ \\
\hline 10 & $\begin{array}{l}\text { Enables "design of policy-driven negotiation protocols" [Amyot03b], with dynamic } \\
\text { components }\end{array}$ \\
\hline
\end{tabular}


Table 2.3 Characteristics of GRL (con't [Amyot03b, Saleh02])

\begin{tabular}{|c|l|}
\hline \multicolumn{2}{|c|}{ Characteristics of GRL } \\
\hline 11 & Supports the identification of not wanted relations among services \\
\hline 12 & $\begin{array}{l}\text { Enables access to the requirements level accessible to the designers, allowing estimates } \\
\text { of future interactions and performance adjustments in a design early phase }\end{array}$ \\
\hline 13 & Makes available options to alter and track the changes to other modeling languages \\
\hline 14 & Provides capabilities to administer changing requirements. \\
\hline
\end{tabular}

GRL is based on concepts classified on four categories, including intentional elements, intentional relations, actors, and non-intentional elements [Amyot02c, Roy07].
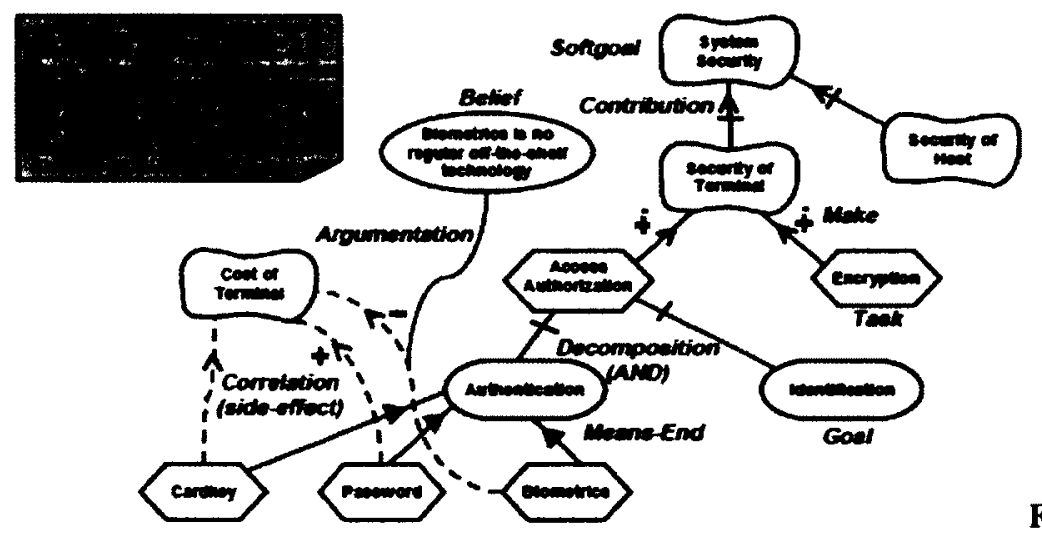

Figure 2.1: Intentional Elements [Amyot02c]

Figure 2.2: Representation Intentional Elements

The elements contribute to system modeling as shown in Figure 2.1 and Figure 2.2 by considering why aspects of requirements, while taking into consideration alternatives, milestones, and explanations. Each element has a unique graphical notation, as described above and specific characteristic, described below: the Intentional Elements have five types of concepts, presented in Table 2.4 , while the Intentional Relations have five classifications, listed in Table 2.5. Goals are important elements explaining why information and specific system functions have been selected to represent the system. They are part of the objectives to be accomplished [Liu01a] with the contribution of 
agents, environment, requirements. Contributions (see Figure 2.3) are valuable relations indicating how intentional elements, relationships links influence the realization level of other elements. The Actors (see Figure 2.4 and Figure 2.5) are aspects able of performing actions that lead to goal completion. To create a GRL diagram, we connect the elements

Table 2.4: Intentional Elements ([Amyot02c, Amyot04])

\begin{tabular}{|c|c|c|}
\hline \multicolumn{3}{|c|}{ Triphon ynements } \\
\hline 1 & Goal & Quantifiable, high-level FR \\
\hline 2 & Softgoal & Qualifiable but unquantifiable requirement, NFR \\
\hline 3 & Task & Solution which achieves goals or satisfice softgoals [Amyot04] \\
\hline 4 & Resource & Entity whose importance is described in terms of its availability \\
\hline 5 & Belief & Rationale/ argumentation associated to a contribution or a relation \\
\hline
\end{tabular}

Table 2.5: Intentional Relations, connecting elements ([Amyot02c, Amyot04])

\begin{tabular}{|c|l|l|}
\hline TIn & & \\
\hline 1 & Contribution & $\begin{array}{l}\text { Link for tasks, softgoals, beliefs [Amyot04], qualified by a degree: } \\
\text { break, some negative, hurt, unknown, help, some positive, make }\end{array}$ \\
\hline 2 & Correlation & Contribution indicating side-effects on other intentional elements \\
\hline 3 & Means-end & Link for tasks achieving goals. alternatives are allowed (OR) \\
\hline 4 & Decomposition & What is needed for a task to be performed (AND) \\
\hline 5 & Dependency & Link between two actors depending on each other \\
\hline
\end{tabular}

through links, according with contributions and relationships. The tasks performed by Users, such as Define Project Plan, Work with Other Departments help materialize highlevel goals, like Report on Activities, Complete Work on System. UCM is the second component of URN, which models FR based on scenarios [Amyot03a, Amyot03b], being

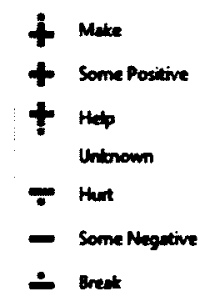

Figure 2.3: Representation of Contributions [Amyot08b]

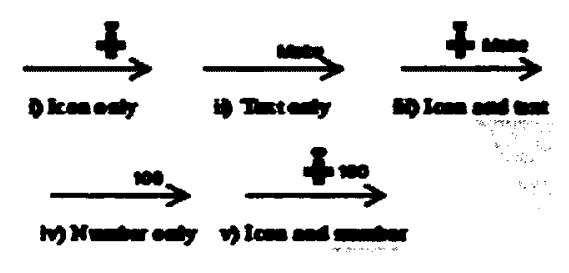

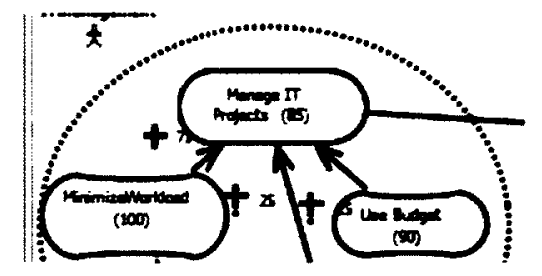

Figure 2.4: Representation of Actor ITManagement 
used to describe relationships among use cases responsibilities [Amyot01a, Amyot04].

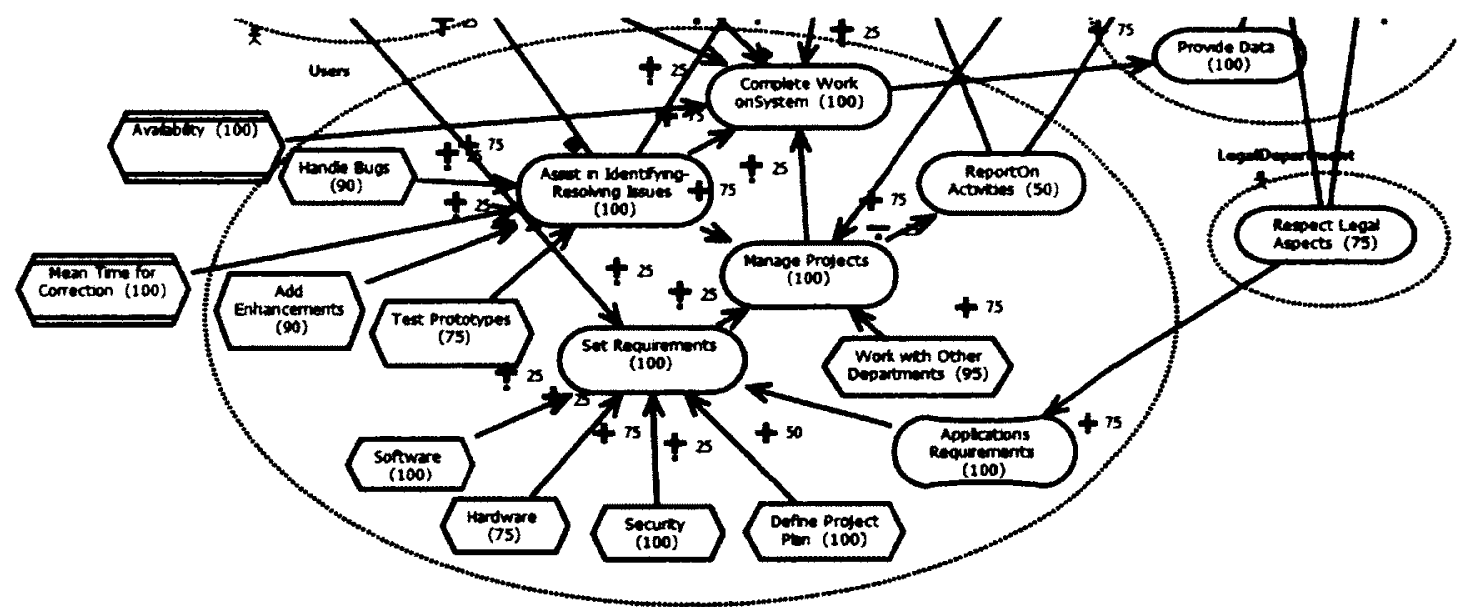

Figure 2.5: Extras from GRL Graph showing Actors, Intentional elements and relations

The UCM. The use cases are presented in a diagram similar with a map, that has the capability of indicating the flow per each scenario selected. Given its characteristics presented in Table 2.6, UCMs are also used to model complex distributed, dynamic systems [Mehta02, Sakkina03] and have the capability [Roy07] to include operational and architectural characteristics. They are also used to validate use cases, model high level designs, generate test cases, "contributing to bridging the cap between requirements and design" [Amyot03a]; to support collection of requirements, designing and testing of system model, allowing modification in the model along with the system development. Modeling with UCM has many applications [Amyot02c] in collecting requirements for mobile distributed systems, in early analysis of performance indicators, identification of unwanted interactions, conversions to other notations [URN10] for performance modeling [Zheng05], test generation [Amyot05a], reverse-engineering [Hamou-Lhadj05]. UCM notations are defined by components summarized in Table 2.7 , which describe casual paths [Mehta02] through relationships among responsibilities [Amyot99a]. 
Table 2.6: UCM Characteristics [A myot99b, Amyot01a, Amyot01b, deBruin01, Liu01a, Amyot02c, Amyot03b, Amyot03c]

\begin{tabular}{|c|l|}
\hline \multicolumn{1}{|c|}{ Characteristics of UCM } \\
\hline 1 & $\begin{array}{l}\text { Represents a graphical modeling and documentation tool, that allows documentation } \\
\text { while still working on the design }\end{array}$ \\
\hline 2 & $\begin{array}{l}\text { Provides more features for describing and analyzing the requirements level than UML; } \\
\text { reduces the gap between the design model and system's requirements }\end{array}$ \\
\hline 3 & Allows the expression of scenarios without needing components that require messaging \\
\hline 4 & $\begin{array}{l}\text { Works with structures and dynamic characteristics, allowing the user to describe static } \\
\text { presentations of dynamic cases, by using dynamic stubs, timers, waiting places } \\
\text { [Amyot01b] }\end{array}$ \\
\hline 5 & $\begin{array}{l}\text { Accepts multiple traces [Amyot99b] and manages accepted and rejected scenarios, as a } \\
\text { helpful attribute for validating test cases }\end{array}$ \\
\hline 6 & $\begin{array}{l}\text { Permits multiple start points in sub-maps, and provide increased support for integrating } \\
\text { the scenarios and viewing the behaviours connected with the structures }\end{array}$ \\
\hline 7 & $\begin{array}{l}\text { Can be used repetitevely and separately, and supports the detection of interactions and } \\
\text { constructions of models }\end{array}$ \\
\hline 8 & Handles early events, allowing participation of managements from various domains \\
\hline 9 & $\begin{array}{l}\text { Facilitates the modeling of the dynamic systems and different alternatives in the } \\
\text { system's architecture, for various behaviours and specific structures }\end{array}$ \\
\hline 10 & $\begin{array}{l}\text { Introduces and integrates in one view behavioural aspects and architectural } \\
\text { characteristics }\end{array}$ \\
\hline 11 & $\begin{array}{l}\text { Has the capability to allow the transformation of the maps into sequence diagrams, test } \\
\text { cases or performance views }\end{array}$ \\
\hline 12 & \begin{tabular}{l} 
Supports performance modelling from requirements and scenarios [deBruin01] \\
\hline
\end{tabular} \\
\hline
\end{tabular}

The relationships are connections created between causes (as pre-established conditions, or activating events), and the effects they create and generated actions [Amyot03c]. The component used to model either a task, function or other activity carried out by the system, is a responsibility. It can be bound to components, and is marked with an " $x$ " (Table 2.7) on the paths (Figure 2.9), which are the lines representing the flow on each map. A path is visually guiding the user through a scenario weaving across the system, from a Start to an End Point, with no need of using messages to bring more details. In many cases the maps are too complex to be modeled in one diagram. Such a case requires layering and using of sub-maps [Liu01a]. A high-level use case map will be used to 
Table 2.7: UCM Components [Amyot02c, Liu01a]

\begin{tabular}{|c|c|c|c|}
\hline \multicolumn{2}{|c|}{ UCM Components } & Details & Eramples \\
\hline 1 & Start Point & Triggers event, showing any condition previously set. & Togin Account \\
\hline 2 & End Point & $\begin{array}{l}\text { Result generated by the cause, known as the post- } \\
\text { condition }\end{array}$ & RegistrationCompl \\
\hline 3 & Responsibility & $\begin{array}{l}\text { Location where procedures, tasks, actions or function, } \\
\text { must be executed }\end{array}$ & RegisterToCourses \\
\hline 4 & Path & $\begin{array}{l}\text { The line connecting a Start Point, going through } \\
\text { Responsibilities(s), up to the End Point. }\end{array}$ & Regixerocoures \\
\hline
\end{tabular}

enclose containers named stubs, that would allow connecting with other sub-maps or plug-ins. They also accept the decomposition, reconstruction of intricate and lengthened scenarios [Amyot02c], being connected to the parent map container through stubs, as shown in Figure 2.6 [Amyot02c]. For example stub Prepare Deployment in Figure 2.8 enables to include alternative maps [Amyot04, Roy07] at a specific location on the path, allowing to model cases that have dynamic behaviors [Amyot02c]. While a static stub accepts only one plug-in, a dynamic stub can have more plug-ins; yet only one plug-in will be selected at the run time, according to the selection policy [Amyot02c]. By using case maps, we can also represent alternative routes - sections of the paths that are shared

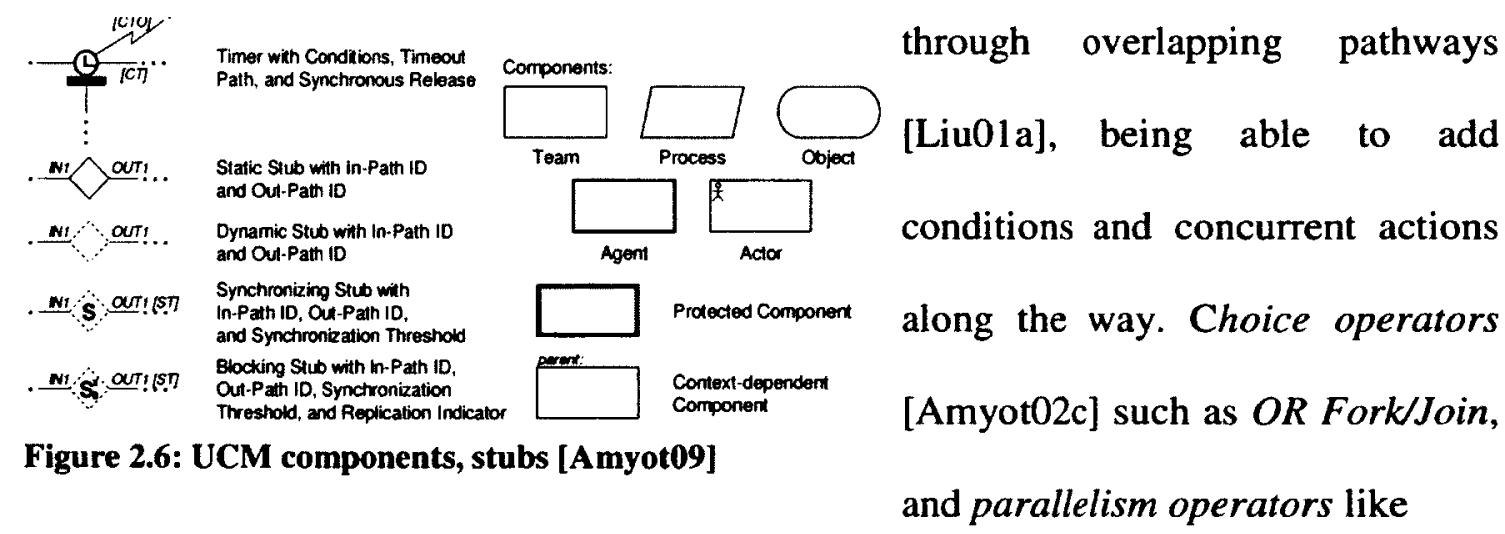

AND Fork/Join (Figure 2.7) allow to model various concurrent paths with different alternatives that connect across the system. For example, they are used to bring together 
at least two common segments and describe the way they synchronize along the path; the $O R$ Fork divides a path into at least two possible routes; the $A N D$ Join executes two ways in the same time, while the $A N D$ Fork divides a path in at least two segments that ran simultaneously. Other elements indicate "waiting places" [Amyot02c] (on hold actions)

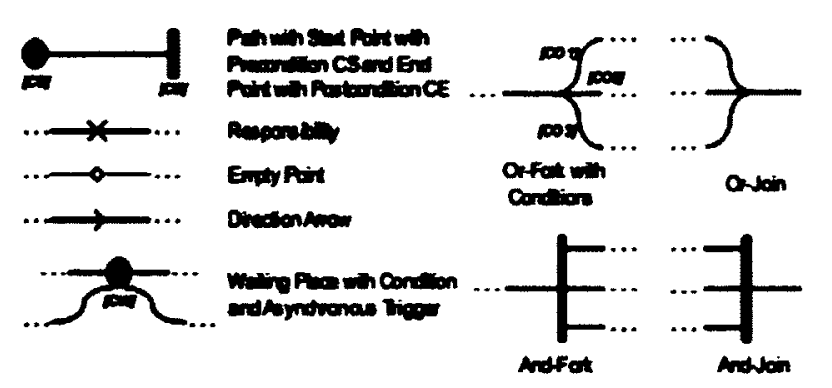
or could introduce timers with pre-

Figure 2.7: UCM components, notations

\section{[Amyot09]}

URN Links - The consolidation of GRL and UCM diagrams in the same graphical view supports the linkage of elements across notations, as the URN Links concept. Their scope is to trace modifications of the GRL and UCM diagrams, to measure the impact of changes on linked elements and on the other model characteristics [Roy07]. The links can connect two model elements from GRL and UCM diagrams and can improve the

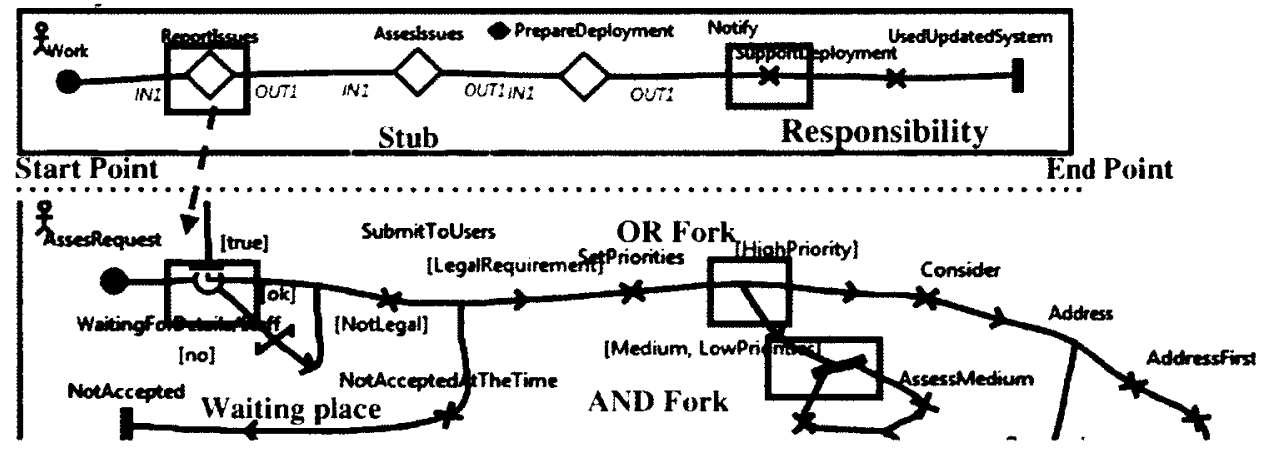

Figure 2.8: - Extras from UCM diagram, depicting UCM components

"consistency between the URN views during model evolution "[Roy07]. The links can be created through a dialog window shown in Figure 2.9; each has from/to elements, 
indicating the start/end point of the Link as per Figure 2.11. Once created, the link is displayed above the linked element as black triangle as shown in Figure 2.10.
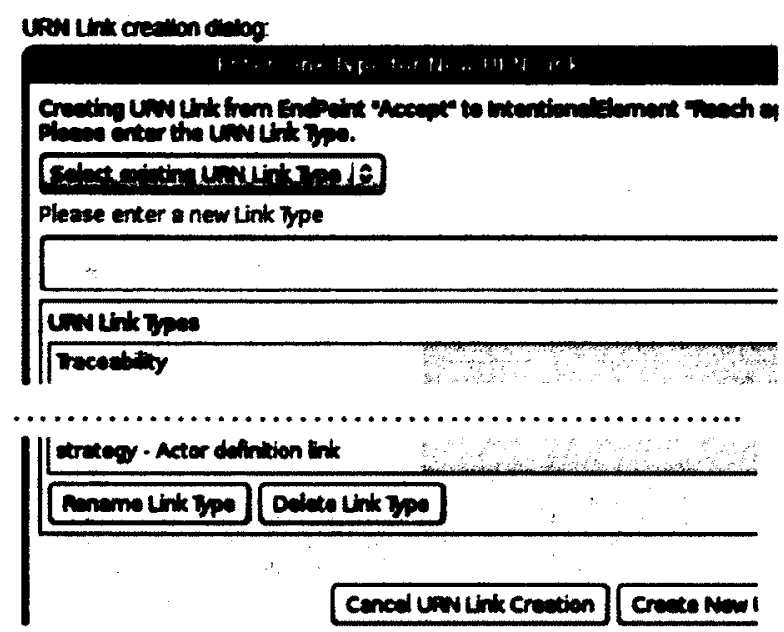

Figure 2.9: URN Link creation view

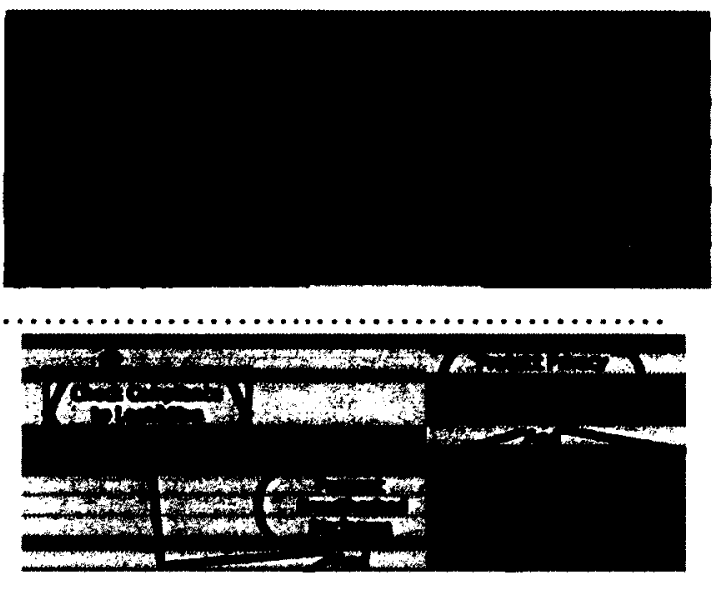

Figure 2.10: URN Link realization

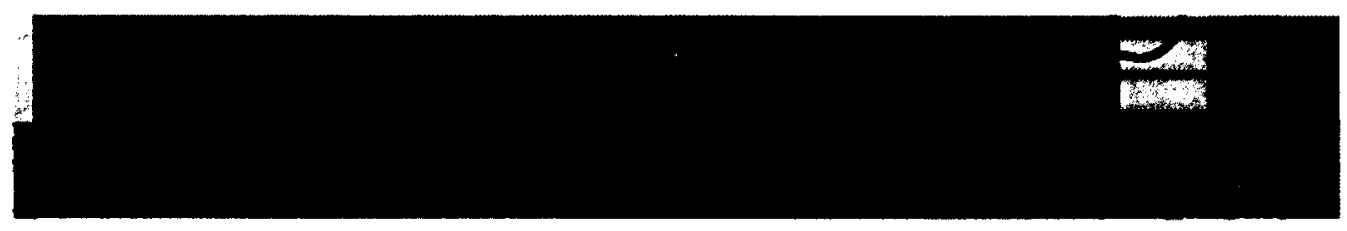

Figure 2.11: Example of URNLinks [jUCMNav12]

The jUCMNav - "jUCMNav is the most comprehensive tool available to date that supports the definition, analysis, transformation, and management of URN requirements engineering models" [Mussbacher09]. It is an Eclipse plug-in, developed to support the ITU-T's URN Z.151, providing graphical editors for both GRL and UCM that support the creation of "links between both views", brings "analysis capabilities (including GRL model evaluations)", allowing different "import and export formats" [jUCMNav12]. A study [Roy07] has looked the contribution of five tools to the realization of goals: one tool has been used to model scenarios, four have edited/analyzed goals, and three tools 
were plug-ins for Eclipse, OpenOME [OpenOME, Yu05], TAOM4E [Bertolini05], jUCMNav [jUCMNava, jUCMNavb].

Table 2.8: Contribution of tools to realization of goals taken from [Roy07]

\begin{tabular}{|c|c|c|c|c|c|c|}
\hline & Com & 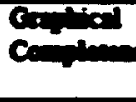 & Dach & Denters & 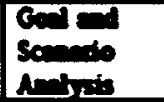 & 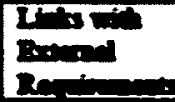 \\
\hline arral & Banch & Exts & & 0'.nen & & En \\
\hline arecmare & Mense & Dant & & Ex & & Urineme \\
\hline Mans & Mon & 8 & $E^{2}$ & 6n!nes & Un'senes & Thes \\
\hline Sonte & Bnt? & Ent & $S$ & Ex & $E x$ & 8 \\
\hline 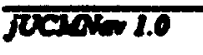 & Es & Es & & & Ens & ES \\
\hline
\end{tabular}

The results of the evaluation have indicated that all notations have contributed to the realization of high-level goals, yet only two solutions, jUCMNav [Kealey05] and TAOM4E [Roy07] have contributed to the Graphical Completeness; the only tool that brought positive contributions for most requirements presented was jUCMNav.

\section{GRL Evaluation and analysis}

The strategies schema has been implemented to facilitate the analysis of GRL models by utilizing evaluations built on various characteristics, including system requirements, design choices, user needs, management participation,business priorities. GRL strategies will use the characteristics to analyze and compare their effects on the model. They can be visualized through the 'Execution View' Figure 2.12. New strategies and evaluation groups can be created, duplicated and deleted in the GRL Evaluation Strategies View. To perform evaluations and run analysis, jUCMNav uses GRL strategies and six evaluation algorithms [Liu03a] (see Figures 2.14, 2.15): qualitative, mixed, formula based, constraint-oriented and conditional, as per Appendix II of [ITU02, ITU03, jUCMNav12]. Most of the strategies sustain a bottom up propagation [Roy07], and the constraint- 


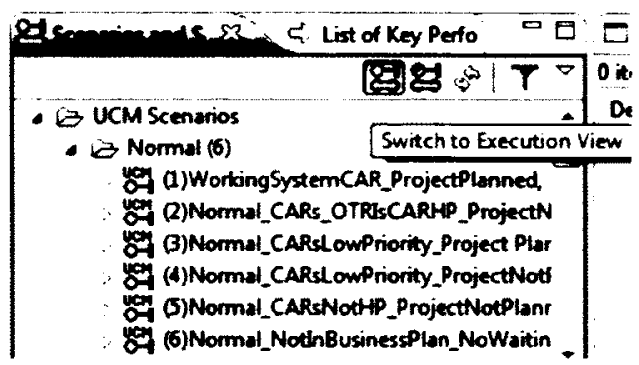

Figure 2.12: Scenarios execution view oriented algorithm is using a constraint solver [Amyot11b]. To proceed with the evaluation, we select the availability or "the satisfaction value [Amyot02c] to low-level intentional elements" [Roy07], tasks and goals. Each GRL

Link can receive a numerical value to indicate the quality and strength of the relation established between two elements. A numerical value, chosen between $(-100)$ and $(+100)$ can be also allocated to a contribution, as a qualitative attribute 'Hurt', 'Make', 'Help'.

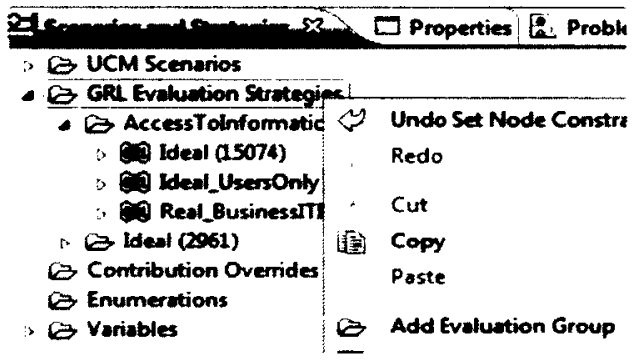

Figure 2.13: Managing strategies
New strategies or strategies that have no

evaluations set by the user, will generate 0 evaluations for actors and intentional elements as presented in Figure 2.16, and Figure 2.17.

[Chung00] will use the value corresponding to their realizations per strategy, to determine the realization of the elements and the satisfaction level [Roy07] reached by each of them [Pengfei07]. The evaluation mechanism that runs the "generic propagation algorithm" [Amyot03e], will then use the links built in the model to generate the satisfaction levels, bottom up [Roy07]. The resulted satisfaction levels will indicate "the impact of decisions on high level softgoals" [Amyot03e] from each goal and actor [Amyot02c, Roy07]. The evaluation result for a goal is calculated only during the evaluation process, and will be displayed on the top of the goal, in accordance with the selected algorithm; it will be a numerical value or a symbol, and will have a color code associated with the satisfaction level. For an Actor, the evaluation shows its global 


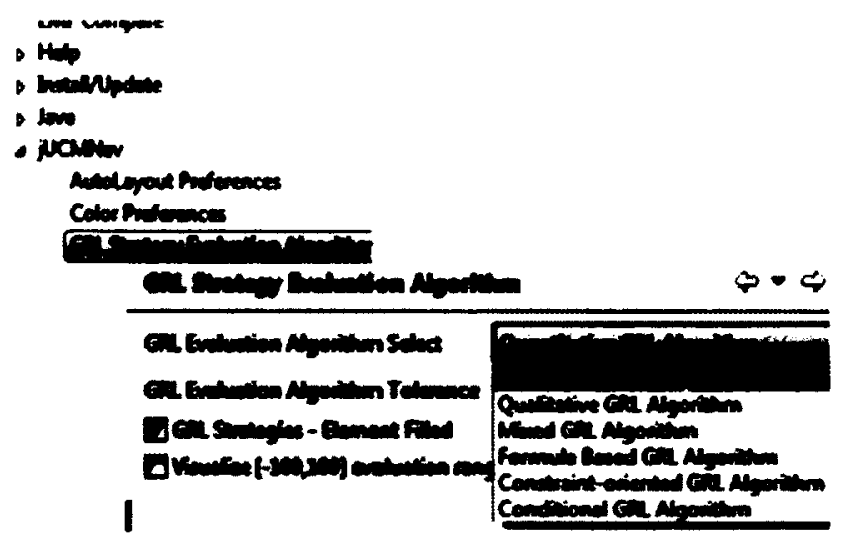

Figure 2.14: Select the evaluation alghoritm

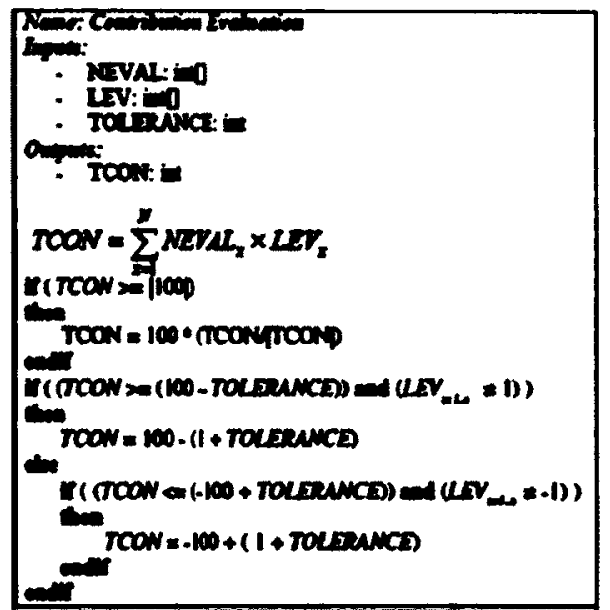

Figure 2.15: Example of an evaluation algorithm [Roy07]

satisfaction. Color red corresponds to satisfaction level 'denied' $(-100)$ shown for goal 'Performance'; green corresponds to $(+100)$ satisfaction level, obtained by goal 'Security of host', while yellow is the color associated with the default value, set to (0) [Roy07].

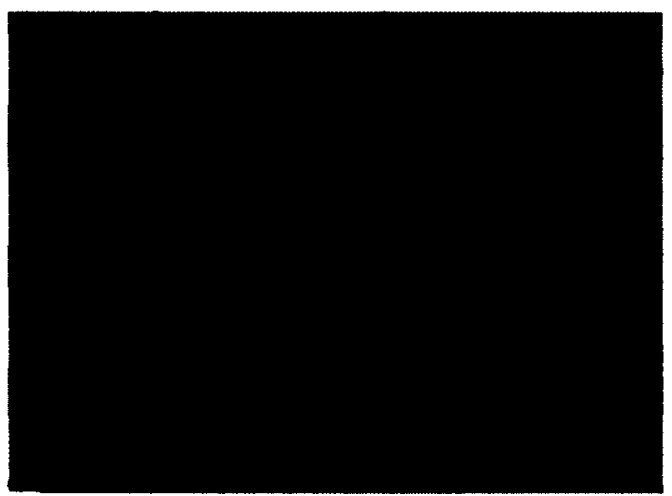

Figure 2.16: Example of evaluation results of Intentional elements

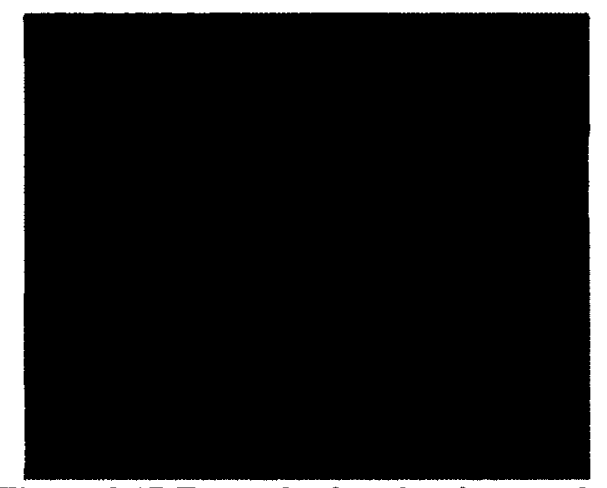

Figure 2.17:Example of evaluation results for an Actor Management

\subsection{The Business Perspective}

The utilization of models as means of description and analysis for business practices and system processes became a focal point in information systems, supporting the analysis and understanding of the business prior adopting technology solutions [Borgida09]. 


\subsubsection{Business Process}

Business model is a framework used in various formal and informal specifications to present the most important business aspects of an organization, including objectives, strategies, structure, processes and guidelines. Next to business aspects, we need to know what processes are needed to meet the business objectives. A business process is a "series or network of value-added activities, performed by their relevant roles or collaborators, to purposefully achieve the common business goal" [Ko09]. While the management of business processes, known as a "holistic management" approach [Brocke10], is used to coordinate business processes of an organization with the demands of their clients, while improving the business processes. Business performance management is "a crossdiscipline with many views, definitions and perspectives" [Ryan09], a set of "management and analytic processes", that allows the business to describe goals, manage and measure performance against the organization's goals as indicated in Figure 2.18. The diagram presents the role of business process management tools in completing business processes; its infrastructure is based on processes that enable financial and

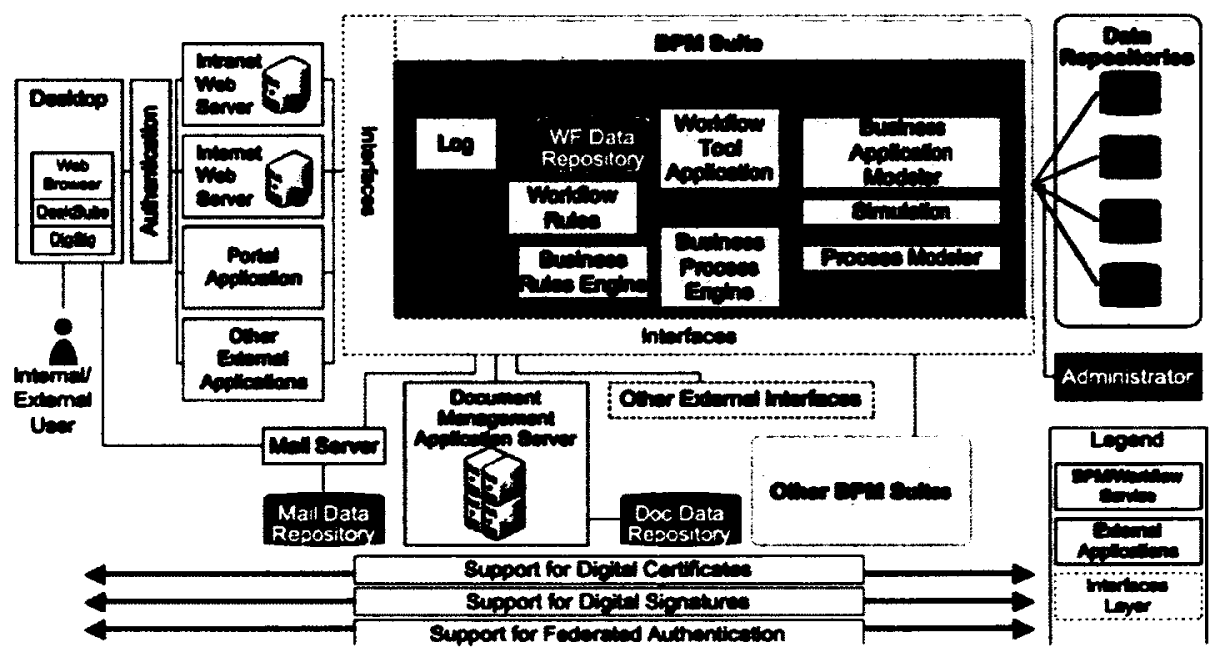

Figure 2.18: Example of Business Process Management (BPM) Service Pattern [NIH07] 
operational planning, business modeling, report analysis and performance monitoring through Key Performance Indicators (KPI) associated to strategies [Brocke10].

\subsubsection{KPI evaluation and analysis}

Business process management has an important role in "supporting business processes using methods, techniques and software to design, enact, control and analyze operational processes involving humans, organizations, applications, documents and other sources of information" [Aalst03]. It contributes to business and organizational efficiency, effectiveness, flexibility, innovation, fusion with technology and customer satisfaction [Kohlbacher09, Vera07]. As demonstrated in [Pengfey07] in order to line up the business goals with the business processes, and to ensure the efficiency and effectiveness of the business, it is recommended to the management/business stakeholders to measure and monitor continuously the organizational business performance by using KPI. They are usually chosen from various measurements that play significant roles in achieving the organization's objectives, have an impact on the organization performance in achieving objectives and indicate if the business processes have been efficient and effective [Kronz06]. KPIs are defined as "financial and nonfinancial measures" [Abujudeh 10], quantifiable, measurable and results-oriented [Wahli07], used to describe and evaluate the level of success of an organization, depending on its strategies and nature, while improving outcomes and reaching out towards the planned goals [Reh12]. The KPI modeling enables the users to define the KPIs (Figure 2.20) that fit with their business and objectives, from characteristics valuable for completing the goals. The KPI model is "a key concept" [Pengfei07] that works with UCMs and GRL specifications to monitor business processes and evaluate business goals (Figure 2.19). Built as addition to the 
GRL extensions, the KPI strategy [Pengfei07] uses the propagation algorithm to identify the impacts of KPIs on intentional elements, while the KPI model asses the "performance management" of "business processes against business goals" [Wahli07]. Once we defined a KPI for an Intentional element (e.g. KPI Cost to Correct, test and deploy from Figure 2.20 has been connected with the softgoal Keep Min Cost), next we can assign to KPI one or more Indicator Groups (Figure 2.20), such as Flexibility, Time, Quality and Cost (for example, KPI Cost to Correct, test and deploy is part of Cost Indicator Group, while KPI Turnaround time is part of the Time Indicator Group (Figure 2.19, Figure 2.20). While working with strategies, we use the evaluation tool, defined " as a percentage value from $(-100)$ to $(+100)$, to measure the satisfaction status of a goal or a task" [Amyot03]. Part of the modeling goal, the Evaluation value for each KPI from a KPI Model Strategy (Figure 2.21) must be asserted against its Target, Threshold and Worst values [Amyot03]. The

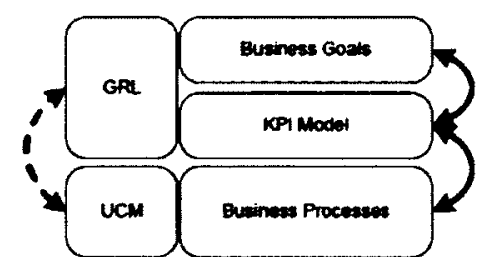

Figure 2.19: URN and business [Pengfei07] target value is used to represent the performance goal, is being accepted as a good case. The worst value is while the threshold is needed to handle warnings; therefore any value between the target and

threshold used to indicate a problem condition; any result located between threshold and the worst value is not accepted and will require corrective actions. The Unit is used to

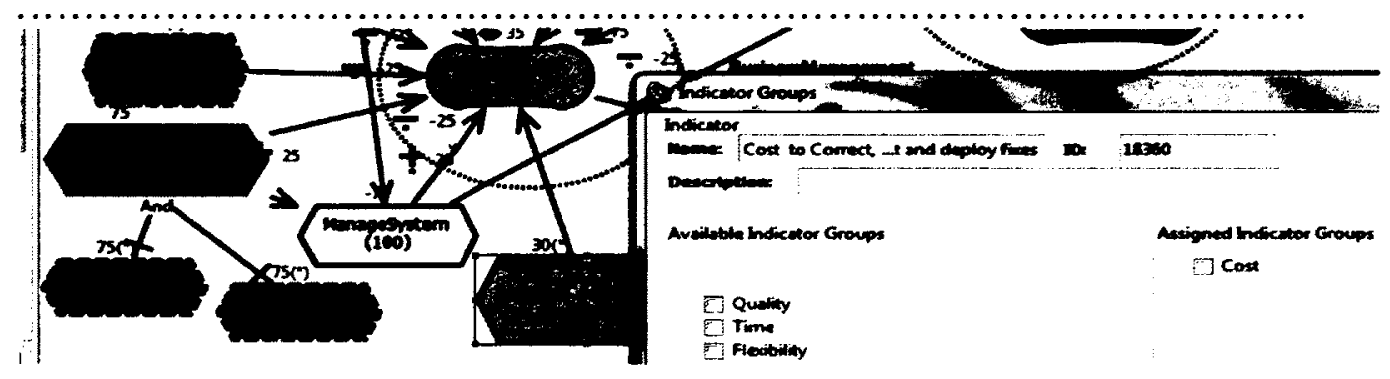

Figure 2.20: Example of Indicator Groups 
indicate the way values are expressed. In [Pengfei07], "KPI values are designed and built in Cognos Report Studio and the monitoring tool and the business information providers are combined through the monitoring Web services", allowing to connect the model with external sources, and to retrieve KPI values "from the back-end data sources, such as business tools, data warehouse, an operational database, or flat files". In [Roy07], links have created between requirements, model and an external tool, to assess how changes in

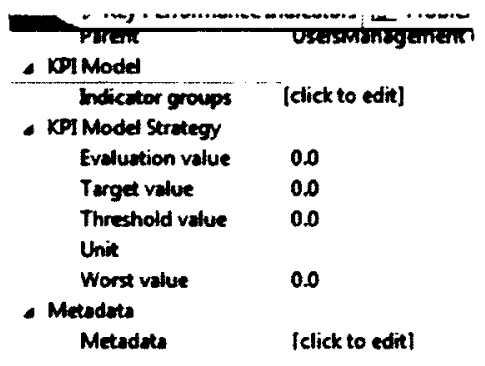

Figure 2.21: KPI Model strategy

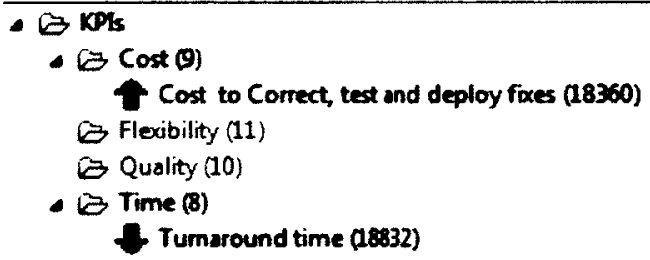

Figure 2.22: KPI 'Turnaround time' has 'Time' as assigned Indicator Group

the model will impact "DOORS objects" [Roy07]. Once the KPI model is evaluated, the users can investigate the KPI impact on the goals realization and satisfaction level, being able to identify the goals that have performance problems/support conflicting processes.

\subsubsection{Modeling the Business}

In engineering and information systems, business modeling is defined [Pengfei07] as a structured method used to present and investigate opportunities; improve the "business objectives of stakeholders", "providers and customers" [Weiss05]; enhance and refine the processes executed in own organization. Business process modeling is used to model processes and goals, being able to handle the five "w" questions [Pengfei07]: "why do this activity, when and where should this activity be performed, what are the responsibilities of this activity, and who should be involved" in it [Weiss05]. In many cases, this process is conducted by management and business analysts to improve the 


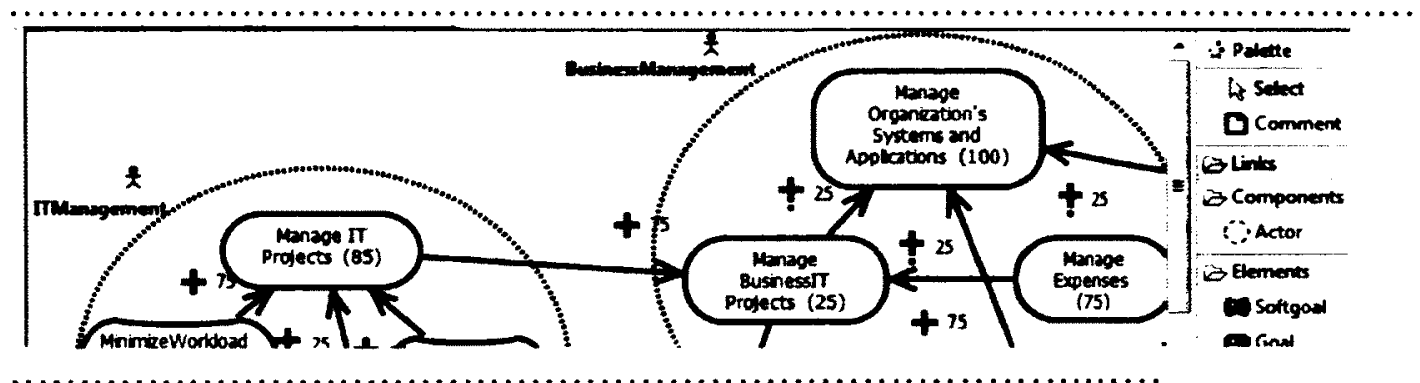

Figure 2.23: Example of Goal Modeling - extract from GRL graph

efficiency and quality of organization's processes. Related studies [Pengfey07, Yu04a]

have indicated URN as the only notation able to bind goal modeling (Fig.2.24) with

business process modeling, using UCM to model the process [Mussbacher07b, Weiss05],

links, to handle relationships, GRL to host business goal models [Pengfei07].

\subsection{Applications of $i^{*}$ and URN models to different domains}

\subsubsection{Modeling with $\mathrm{i}^{*}$}

The $\mathrm{i}^{*}$ model has been used in different types of modeling, including information systems [Liu03a], privacy, and security [Liu03b], redesign of business processes [Chung00] and "aspect-oriented" systems [Roy07, Yu04b]. The i*supports applications (see Table 2.9), from small to complex systems, and model notations like Visio Technical and jUCMNav.

Table 2.9: Applications of the $i^{*}$ modeling tool [Amyot03a, Kethers05, Maiden04]

\begin{tabular}{|c|c|c|}
\hline \multirow{2}{*}{\multicolumn{3}{|c|}{$\begin{array}{l}\text { Domain } / \text { peetits } \\
\text { 1.Requirements Engineering }\end{array}$}} \\
\hline & & \\
\hline $\begin{array}{l}\text { Defines requirements } \\
\text { for social, complex } \\
\text { environments [Yu09] }\end{array}$ & $\begin{array}{l}\text { Applications for business processes, } \\
\text { government e- applications, health care } \\
\text { [Kethers05], air traffic [Maiden04], for } \\
\text { industry [Petit99],in [Amyot03a] for } \\
\text { telecommunications". }\end{array}$ & $\begin{array}{l}\text { Study of management requirements } \\
\text { for a system that administers flight } \\
\text { scheduling and manages the } \\
\text { departures for airports in Europe } \\
\text { [Maiden04]. }\end{array}$ \\
\hline \multicolumn{3}{|l|}{ 2. Social modeling } \\
\hline & $\begin{array}{l}\text { Social modeling can be successfully } \\
\text { used in any software developments } \\
\text { lifecycle phases successfully used in } \\
\text { any software developments lifecycle } \\
\text { phases }\end{array}$ & $\begin{array}{l}\text { Applications have been developed to } \\
\text { manage computing [Briand98], } \\
\text { industry processes, database design, } \\
\text { information, and [Yu09]business } \\
\text { intelligence [Pourshahid08] }\end{array}$ \\
\hline
\end{tabular}


Table 2.9: Applications of the $i^{*}$ modeling tool

\begin{tabular}{|c|c|c|}
\hline Domain / Details & Description & Applications \\
\hline & $\begin{array}{l}\text { Social modeling can be successfully } \\
\text { used in any software developments } \\
\text { lifecycle phases successfully used in } \\
\text { any software developments lifecycle } \\
\text { phases }\end{array}$ & \\
\hline \multicolumn{3}{|c|}{ 3. Enterprise Engineering } \\
\hline $\begin{array}{l}\text { Enterprise } \\
\text { architecture }\end{array}$ & $\begin{array}{l}\text { "A systematic" approach [Zachman87] } \\
\text { has been adopted to manage } \\
\text { component systems in an enterprise, } \\
\text { instead of one system at the time } \\
\text { [Yu09] }\end{array}$ & $\begin{array}{l}\text { This approach allows the model to } \\
\text { reveal and analyze various aspects } \\
\text { characteristics to the business } \\
\text { domain, such as [Rifaut08] [Yu09] }\end{array}$ \\
\hline $\begin{array}{l}\text { Business model } \\
\text { innovations [Yu09] }\end{array}$ & $\begin{array}{l}\text { Most of the modeling methods adopted } \\
\text { by business processing are coming } \\
\text { from system }\end{array}$ & $\begin{array}{l}\text { Business models and business } \\
\text { processing }\end{array}$ \\
\hline $\begin{array}{l}\text { Mobile and Internet } \\
\text { technologies [Yu09] }\end{array}$ & $\begin{array}{l}\text { Various and many techniques have } \\
\text { been used, from simple flowcharts, up } \\
\text { to [BPMN06] connecting business } \\
\text { goals with business processes }\end{array}$ & $\begin{array}{l}\text { Can model not only the interaction } \\
\text { between IT and business analysts, but } \\
\text { also to identify management's goals } \\
\text { affected [Yu04a] by additions and } \\
\text { modifications in the system. }\end{array}$ \\
\hline \multicolumn{3}{|l|}{4 Security } \\
\hline $\begin{array}{l}\text { Social models can be } \\
\text { also used to handle } \\
\text { security concerns, } \\
\text { including trust and } \\
\text { privacy [Yu09] "in an } \\
\text { engineer process" }\end{array}$ & $\begin{array}{l}\text { Security concerns, like [Yu09] } \\
\text { "confidentiality, integrity, availability, } \\
\text { can be modeled as soft-goals, and used } \\
\text { at the system level as firewalls, } \\
\text { encryption-decryption tools }\end{array}$ & $\begin{array}{l}\text { The Guardian Angel Project [Yu02], } \\
\text { [Yu03] is a complex combination of } \\
\text { applications delivered for a health } \\
\text { care system [Szolovits94]. Modeling } \\
\text { has been also used to analyze privacy } \\
\text { of data, establish client's trust, } \\
\text { securityn [Elahi03], [Liu03b]. }\end{array}$ \\
\hline
\end{tabular}

\subsubsection{Applications of the URN model to different domains}

"URN is the first and currently only standard which explicitly addresses goals in addition to scenarios" [Bochmann 10]. According to a recent study [Amyot l 1a] supported by over 280 academic publications on URN, a significant shift has been observed from the traditional use of URN in telecommunications and responsive systems towards the management of business processes and software development. URN modeling has been used in various solutions, as presented in (Table 2.10). 
Table 2.10: Applications of the URN modeling tool [Amyot05a, Amyot08a,Monkewich01]

\begin{tabular}{|c|c|c|}
\hline Applications & Details & W Deserptipn \\
\hline $\begin{array}{l}\text { 1.Tele- } \\
\text { communications }\end{array}$ & $\begin{array}{l}\text { Design, development } \\
\text { of Standards, Services }\end{array}$ & $\begin{array}{l}\text { URN has been used to model projects for Wireless } \\
\text { Intelligent Networks [Amyot08a], and dynamic } \\
\text { protocols used in routing IP networks } \\
\text { [Monkewich01] }\end{array}$ \\
\hline $\begin{array}{l}\text { 2. Web systems, } \\
\text { electronic- } \\
\text { commerce } \\
\text { systems }\end{array}$ & 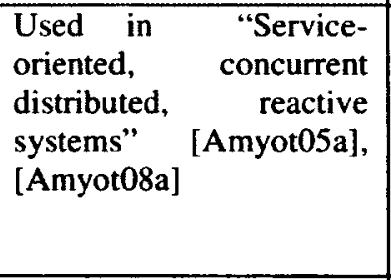 & $\begin{array}{l}\text { By modeling important capabilities [Jacque04] of } \\
\text { an on-line web system, the user was able to identify } \\
\text { hidden errors, passed undiscovered when the } \\
\text { traditional testing methods have been used. Then } \\
\text { for a hotel reservation system, the[Billard04] } \\
\text { modeling was used to bring benefits at both } \\
\text { notational and process level }\end{array}$ \\
\hline $\begin{array}{l}\text { 3. Health } \\
\text { applications }\end{array}$ & $\begin{array}{l}\text { Indicate improvement in } \\
\text { monitoring business } \\
\text { aspects, processes and } \\
\text { the management of } \\
\text { performance }\end{array}$ & $\begin{array}{l}\text { In this project [Pourshahid08] different challenges } \\
\text { have been imposed by the way the access to } \\
\text { information database has been granted; they have } \\
\text { been modeled with UCM for a large research } \\
\text { hospital from Ontario. }\end{array}$ \\
\hline $\begin{array}{l}\text { 4.Modelling } \\
\text { Business } \\
\text { Processes }\end{array}$ & $\begin{array}{l}\text { [Pourshahid08] is a good } \\
\text { example of a study } \\
\text { presenting the GRL and } \\
\text { jUCMNav [Amyot } 08 \text { ] ] } \\
\text { with KPI enhancements }\end{array}$ & $\begin{array}{l}\text { This analysis describes the business process, } \\
\text { showing improvement in connecting various } \\
\text { scenarios with business goals, in extending the } \\
\text { strategies, to connect with external data sources and } \\
\text { monitoring tools [Ghanavati07] (like DOORS), in } \\
\text { identifying the business processes realized and the } \\
\text { policies used are in compliance with legislations } \\
\text { and regulations }\end{array}$ \\
\hline $\begin{array}{l}\text { 5.Analysis and } \\
\text { transformations }\end{array}$ & $\begin{array}{l}\text { Presents approaches of } \\
\text { different analysis and } \\
\text { transformations }\end{array}$ & $\begin{array}{l}\text { Transformations are analyzed from the perspective } \\
\text { of testing the goals of the models created with } \\
\text { UCM [Amyot05a], [Amyot05b]. }\end{array}$ \\
\hline $\begin{array}{l}\text { 6.User interface } \\
\text { requirements }\end{array}$ & $\begin{array}{l}\text { Specifications, analysis } \\
\text { [Amyot08a] of user } \\
\text { interface requirements }\end{array}$ & $\begin{array}{l}\text { This case is using RE and scenarios for user } \\
\text { interfaces, demonstrating that is possible to build } \\
\text { better systems if effort is put into developing users } \\
\text { interfaces to depict and feed on corresponding } \\
\text { user's tasks [Alsumait04] }\end{array}$ \\
\hline $\begin{array}{l}\text { 7.Patterns } \\
\text { formalization with } \\
\text { URN }\end{array}$ & $\begin{array}{l}\text { Enables "rigorous trade - } \\
\text { off analysis" while } \\
\text { "maintaining the } \\
\text { genericity of the solution } \\
\text { description" } \\
\text { [Mussbacher07] }\end{array}$ & $\begin{array}{l}\text { It is essential to present a pattern in such a manner } \\
\text { that will allow the user to appreciate if the } \\
\text { resolution made available to him will help resolve } \\
\text { his problem [Mussbacher07] }\end{array}$ \\
\hline $\begin{array}{l}\text { 8. Reverse- } \\
\text { engineering, } \\
\text { re-engineering } \\
\text { contexts }\end{array}$ & $\begin{array}{l}\text { "Formal semantics and } \\
\text { time extensions" have } \\
\text { been studied with UCM, } \\
\text { [Hassine05] [Hassine07] }\end{array}$ & $\begin{array}{l}\text { Other studies have researched if is possible to } \\
\text { recuperate UCM scenarios from "execution traces" } \\
\text { [Hamou-Lhadj05] and programming code } \\
\text { [Amyot02b]. }\end{array}$ \\
\hline
\end{tabular}




\section{Chapter: Working System Model}

This chapter presents the URN model of the case-study system, which is inspired from a reality. The system is described first, then the GRL and UCM models are presented next.

\subsection{Description of the Case Study System}

The system we are looking to model is a component of a complex distributed system that serves a large scale enterprise (country-wide) known as the Organization (Figure 3.1). The Organization is composed of many different Sub-organizations (SO), which have their own operational mandates, managers, receive distinct budgets and operate with their own staff members (which could range from 2 - 3 employees up to 15 - 20). Many SOs are sharing the Organization's network, as well as corporate services and applications, including legal and regulatory services provided by the Legal Department (LD) and the Regulatory Department (RD), business administration and technology services [Sage01], [Ulrik10] provided by the Internet Technology Department (ITD) and Business Department ( $B D)$. Some SOs, such as SO (3) from Figure 3.1, manage the IT technology and business administration services with their own equipment and staff. Often SOs are involved in internal inter-departmental projects across the organization, and in external projects at national level, with different clients and various organizations. These types of projects are based on contracts, memoranda of understanding, and other legal agreements established between the participants. The SO of interest for this case study is the Users Department or the Users. The roles of the users from this particular SO, include receiving of tens of thousands of data records on an annual basis from various clients; responding to thousands of requests from external clients (which include companies, universities and 
the Canadian public); providing data reports for the use of the public, as well as ad-hoc reports requested by some clients, including regulatory departments, the organization's stakeholders, universities. The system presented in this cases study has the following characteristics: it has been designed to meet the Users work requirements and mandatory duties; has a large database that contains millions of records collected over the years; it

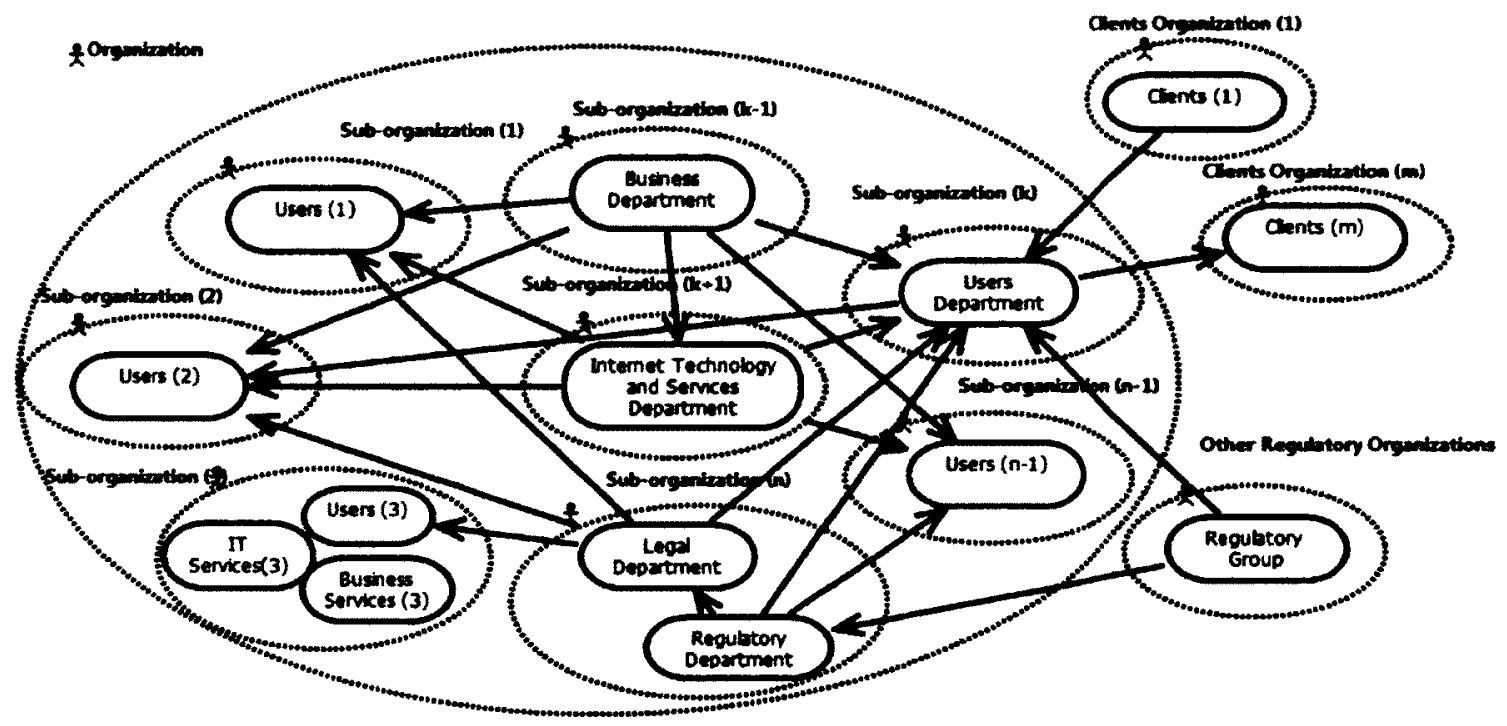

Figure 3.1: Example High-level view of Organization (n, a positive integer at least greater than 3)

requires monitoring, management and maintenance work throughout its life cycle. The current implementation is quite new, as it went through a refurbishment-process a few years ago. The old technology of the system had to be decommissioned, new technology and software applications installed.

\subsubsection{System Actors}

The distinct SOs which are part of this case study, and also describe the main actors of the system, are the Users $S O, B D, I T D, L D$, as presented in Figure 3.1. The users from each SO work on corresponding sub-network segments, which are components of the corporate network established at the organization level. The corporate network is managed by ITD for technology and applications, and by BD for administration of 
business information and activities. The users from each SO work with their own operational plans, have own responsibilities, deadlines and objectives to meet. Interdepartmental work relationships have been established and maintained between SO and $\mathrm{LD}, \mathrm{RD}$ in some cases, given that these SOs provide legal and regulatory services to all SOs, advising the users on any legal matters related to requirements, environment and legal aspects. They also provide support in revising documentation, contracts and clauses that have legal and regulatory consequences on the organization and its business; in imposing regulations and rules on different administrative businesses, aspects and environments, for the public's wide interest and benefit, including serving all the users' privacy and security, safety and health concerns. Each SO has its own set of employees or users, objectives to be met, various and unique responsibilities on own deadlines, own and independent budget and management. Each SO has a manager which regularly reports to the high-level management, also known as Stakeholders.

The Users mandatory activities include: receiving data records from various Clients; providing data records back to the Clients, without sharing data between any of the Clients and without providing data submitted by certain Clients to other Clients that have no connection and no involvement with that type of information; respecting rules and regulations on privacy and security of information; providing data reports on annual basis for the use of the public, as well as ad-hoc reports requested by some clients, including the $\mathrm{RD}$, the organization's stakeholders, universities or external companies that submitted data and are looking for certain correlations with previous data records. The mandatory activities require the Users to provide information to Clients upon receiving requests, and to support the RD in their activities. The Clients that work with the Users on a regular 
basis can be classified in the following categories: Clients that submit data records to the Users; Clients that request data reports on the data submitted to the Users; organizations that are submitting records to the Users SO as part of their activities; companies that require data information related to their own employees in accordance to laws and related regulations; Canadian public that can ask for the summary of the records and the data accumulated over the years; RD and other regulatory agencies, that ask for regular reports on the clients submitted data and on other matters; other organizations and universities from across the country, that could also ask for reports or participate in inter-comparison studies. Some clients submit tens or thousands of records, while others submit up tens of thousands per year. There are many activities running in parallel at organizational level. As illustrated in Figure 3.1 the LD/internal RD, as well as the ITD and BD are constantly working with some of their internal SO clients (indicated by the arrow at the end of the connection link), while others, like the Users from $\mathrm{SO}(\mathrm{k})$, are diligently accepting records from clients, or providing information to the clients. As previously mentioned, the Users system is currently managed by the ITD and BD; the group from the ITD that is taking care of specific system components at an organizational level, is composed of programmers and database, network and systems administrators; while the ITD group that is managing the network segment shared by all users across the organization, the intranet, is composed of tens of programmers and testers distributed in offices across the country. $\mathrm{BD}$ also is composed of business analysts, project and programs managers. The Users have limited information on the way these groups operate and how they manage the Users system; the Users usual have assigned a business analyst and one developer to work with, while keeping all management (i.e., Users SO, ITD and BD) informed. 


\subsubsection{Users connections with other sub-organizations}

Services and applications running on the Users system, front end and back end, including production, development (indicated in Figure 3.2) in the Users domain of responsibilities, database, code, monitoring, back-up, recovery, security, hardware equipment, technology, are managed by the ITDSC of ITD. The organizational and administrative aspects of the system's business, including system and SO requirements, also indicated in Figure 3.2, system and business requirements documentation, system architecture, design, development and implementation, deployments, corrective action requests, use cases,

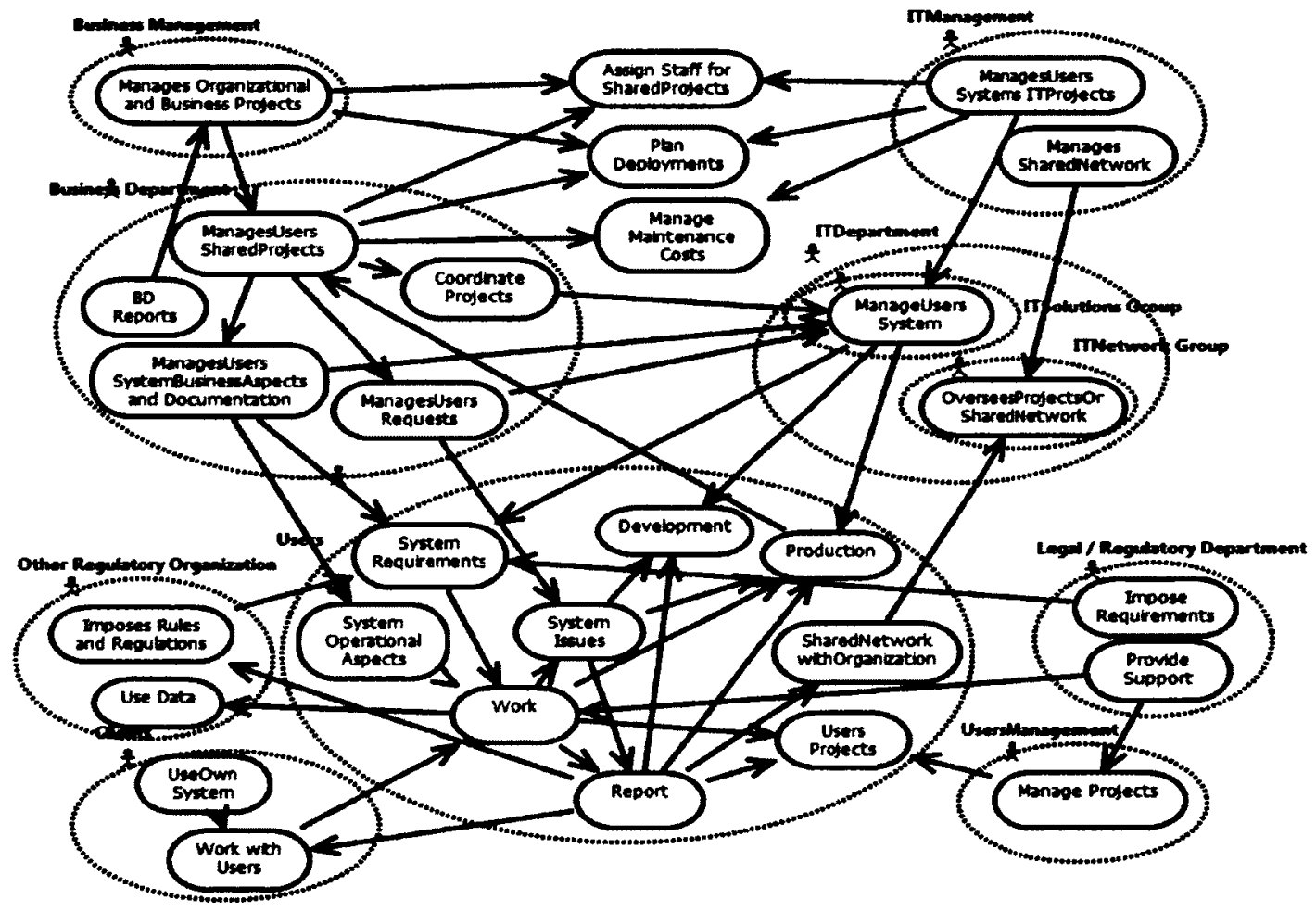

Figure 3.2: Example Presentation of services/activities managed by ITD and BD

system issues, corrective actions, use cases, test cases, test results, are managed by BD.ITD and $\mathrm{BD}$ are also involved in administering inter-departmental projects, being in charge with staff assignments, projects management, coordination of deployments, while LD provides legal support and RD assistance in regulatory activities and related matters. 


\subsubsection{Characteristics of users activities}

The Users work with complex applications hosted on their system, which are also running on a sub-network of the corporate system, set up at the organization level. The Users system allows for restricted access to the employees of the following SOs: Users SO and their manager, some staff from BD and ITD, and the IT Solutions Group (ITSG) the part of ITD that manages systems and applications of other SO. The system used by Users has two components: the Network Component (NC), or the equivalent of an intranet network, and the Specific Component (SC) or the equivalent of an extranet component. The NC is the part of the network used at Organization level, and shared by users from different SO. This particular system component is using a shared network segment allowing the users from various SO to communicate exchange and store information across the Organization; none of the external clients have access to the NC.

The second component of the Users system is the SC, and has restricted access to users according to designated applications and databases. The limited access to the SC is based on Users requirements, with respect to privacy and security issues, and is granted only to Users, UM, assigned Users Clients and staff members from the LD. The administration of the SC of the system falls under the responsibilities of ITD and BD. The ITD located in another SO, has two component groups: the IT Network Group (ITDNG), which is managing the NC of the system, and the IT Solutions Group (ITDSG), which is in charge with the maintenance of the SC, with everything that means front-end, back-end applications and networking, including system operational aspects, environment for software applications, hardware components, system monitoring, database design, development and administration, code maintenance, development of new functionalities, 
bugs corrections, testing and deployments. The ITDSG also administers performance and security aspects, managing the NC system, including security requirements for this network segment, system network requests, speed and response time, storage and backup, etc. The NC must adhere to specific security standards and implement certain techniques imposed by the Privacy and LD, own ITD, BD and clients. According to the organizational and operational business established at the corporate level, the Users have limited administrative rights on the SC. They must access and use the system for daily work, and this mainly includes some management of users roles, some triggering of transactions, while excluding system monitoring, access to software and hardware equipment, 24h service support, etc. Basically the Users have no access to "overall operations performance improvements resulting from new processes and information services", to any "selected processes for specific technical changes" [Zheng06] or to knowledge on "bottlenecks resulting from peak workload, staffing, and other resource limitations" that have been "identified "[Zheng06]. For such a complex use case system, we "understand the need to align organizational technology and business objectives and aim to articulate this vision to employees, key partners, and external stakeholders" [Philbinl1]. We also understand that such a process will challenge the organizational technology and business characteristics, in order to ensure the realization of goals at a global level, while still realizing at a satisfactory level the goals from each suborganization. According to [Damian07] "the process differences inherent in interorganizational partnerships lead to difficulties in aligning RE processes and supporting tools, preventing knowledge management and change management practices from being effectively implemented across sites". The challenge is given by the way the SOs are 
organized: they have own operational technologies, system and applications needs, own business activities, projects and goals, own operational plans, responsibilities, own ranking system and values for sorting priorities and requests received from the clients; they have own project plans, deadlines, human and computing resources to handle work projects and budget.

\section{Characteristics of Inter-departmental work}

Working across each sub-organization's boundary on a new common ground towards the completion of inter-departmental projects is complex (as shown in Fig. 3.2) and might require a temporary integration of sub-organization's frameworks and convergence of technologies, and "the required level of organizational and technical interoperability is still a major factor in multi-agency missions" [Zheng06]. We understand that it might be demanding for different entities (e.g. SOs or departments) to perform well in ad-hoc projects; the participants are coming from different environments with their own mandates, background and objectives, and they will have to work on new grounds, in temporary environments established across organizations, as they need to learn to build work relationships, to trust each other and coordinate their efforts towards common goals. We expect that this will require efforts from all participants, challenging their SO on different levels, such as project planning, staffing, budgeting, and could have an impact on their own work and objectives. The inter-departmental work supports indeed the realization of common projects, while it challenges each SO in return, on various aspects:

- Communication language: the participants are coming from different SO, have diverse backgrounds and different work objectives. This could create a challenging 
communication between participants in common projects, until they agree on common terms that have the same meanings for all participants. Studies are indicating that "differing attitudes toward hierarchy and communication styles (for example, not being able to openly communicate project priorities or progress) often" can result in "misinterpretation of actions, leading to damaged trust relationships" [Damian07].

- Communication process: the process is quite complex, and frequently requires the exchange of many notifications, requests, approval for requests, communication inside of the SO, with their own management and staff, and outside of the organization, with management, BD, ITD, BM, ITDM, LD, Clients. Often, communication at a higher level involves managers reporting to Stakeholders, and sometimes Users reporting to management. In most of the cases, there is an additional time delay added to the acknowledgement, response time, completion of the project, "time delays in both the communications network and the decision processes that control organizational response" [Zheng06]. Sharing and integration of information across SO and working groups "can become problematic. Business users and managers can fail to share information about the client organization's priorities, standards, and policies" [Damian07].

- Objectives to fulfill: in case the work for the common projects takes priority, the own objectives might need to be reassessed or priorities might need to be revised to ensure that all goals will be realized accordingly.

- Own deadlines to meet: depending of the common work to be completed, some of the deadlines set either for own projects or for the common projects might be overdue and results could be delivered with delays.

- Ad-hoc or urgent requests: must need to fit in the current work; they would become 
the objectives with the highest priority, taking over on the other goals and tasks.

- Coordination and delegation of work: is very important in inter-departmental projects to ensure that is well understood right from the beginning who does what, how, and for when; to assure that objectives will be completed as planned, and to avoid duplication work, in at least the following areas: project planning, quality management, documentation of processes and work completed.

- Staffing: the participants required by common projects across the organization, are coming from different groups, internal or external departments. Often the same staff members are involved in own SO projects, as well as in inter-departmental work. This might require reassessment of the work plans and tasks, to be able to mitigate potential conflict of interest with regards to all objectives to be met, to avoid staff burden, to manage potential overtime, due to the work load, to estimate possible delays of deliverables and how they can be overcame. Other challenges might be given by the effort of finding the right people with the right knowledge and experience to fit in the common project, and to ensure their participation upon completion. Often people change jobs/positions, therefore one department could have no more participants for the common projects, and also no budget to hire a contractor to handle the work for completion

- Handling of budget: how will the common project get funded; will it be done with shared participation from each sub-organization or will require financial contribution only from some clients; what will happen with unplanned or urgent projects that have no assigned budget, and cannot be financed by other sources.

- Corporate knowledge: invested in inter-departmental projects should be preserved by each participant; information should be constantly shared in between participants, to 
avoid situations in which they could "fail to reveal accurate information about development estimates and priorities" [Damian07].

- Collaboration: work relations and common projects with some other SO might be still required for current projects, as well as for future activities. They should be included in the coming operational plans and budgets required for the next years. For example, the Users system is maintained and administered by ITD and BD, therefore future work that requires assistance and participants from these SO should be planed accordingly. We believe that such planning should be made at the level of each SO participant, such as Users, as well as at a global level for ITD, BD, given that they probably handle tens of clients like the Users. There are situations that can be estimated and evaluate in advance, to ensure a good work collaboration. For example, what happens when contradictory intentions, yet aligned with own SO' objectives, develop across the SO's boundaries, across the organization, are supporting the objectives of some SOs while could be of disadvantage for other goals. In some cases, the planning of future collaboration with BD and ITD proved to be challenging for the Users; for example, how could they estimate any future correction work required by the system when they have limited access to system monitoring and administration. What happens when the priorities set by the Users to their requests will be ranked by $\mathrm{BD}$ lower than the priorities received by requests addressed by other SO. We believe that modeling such cases will help align the work required by inter-departmental projects with the SO's own goals and project planning; it could help verify the process and identify potential conflicting situations that could have been avoided, mitigated or excluded; it could ensure that each SO's priorities are well understood and considered, and that goals will be reached at a satisfactory level for all. 


\subsection{System Requirements}

Our focus is on a subset of the system requirements that are concerned with the following issues: in which way the operation of the system is organized, how different departments involved in the system activities interact and what is the impact on the realization of the goals, how is the information transmitted between different operational modules across the organization, what type of processes and reporting loops have been implemented. We want to understand how the administrative information is being monitored at system level and learn how system matters are being reported to system administration, management, users and clients; determine who will address and initiate corrective actions requests and how they will propagate across the organization; identify who needs to know what for a distributed system model that has different managers on component modules. In distributed systems, there are various inter-departmental projects developed between SOs at the organization level, in which the participants "must specify and manage requirements across cultural" boundaries. While the Users workstations are located in the Users SO, software programs, user's applications, hardware devices, and network components, including routers, hubs, switches, communication channels, servers, backup, archiving are managed by ITD across the enterprise. This way, the users system's "functionality can be described by availability of the services for end users" [Sladic11]. Each system's functionality can be affected if "anything goes wrong with any of the

software applications or hardware equipment" and if "there are problems regarding functionality [Ho08] of network elements", "failure and malfunctioning" [Sladic11].

\section{Users' view on system requirements}

The SC of the Users system shall allow access through a web portal to applications and 
information only to authorized Users; the data stored in the database shall be available any time during work hours and in any urgent situations. The information in the databse shall be updated automatically when the Clients submit data records, and any time the Users enter data in the system. Only Users with manager role can enter data in the database. The maintenance of the SC should be done automatically in such a way to assure that the system is up and running $24 \mathrm{~h} / 7$. The system must perform the designed workflows, must operate according to the regulatory requirements, and must perform different sets of operations. The screens accessed by the Clients have to be restricted, so client's data cannot be shared across different clients. For certain data types, the records are accepted with dates before the current upload date. Changes to the system can be done only after receiving approval from the Users, which shall request approval from UM in order to proceed. Correction actions shall be addressed only by the BD, which will further inform the ITD. Users with 'data entry role' are allowed to print and fax reports but cannot process any data records; users with 'regulatory role' can read data records submitted by any Clients; only users with 'administrator role' can delete records only if joint appoval has been received from UM, Stakeholders, LD and the client that submitted the data. Clicking the 'manually Upload File' button, moves the file from the external waiting queue to the system processing workflow. All the personnel that has access and uses the system must be trained according Users policies and procedures. The database must have the audit trail enabled, any default database passwords shall be disabled. The SC shall have enough bandwidth to be able to function regardless of traffic on the other network segments across the Organization. The system shall be accessible at any times only by assigned Users, shall be backed up in real-time, shall be in compliance with rules 
and regulations dictated by the corresponding authorities, regulations. The system shall have its documentation and any changes in requirements updated by the $\mathrm{BD}$; system deployments shall be also tracked and documented with their details, by BD. The system's changes shall be deployed by ITDSG without disturbing the functionality of the SC; any system deployments shall be coordinated by $\mathrm{BD}$ with the ITD to ensure successful operations. The system shall be legal, maintainable, modifiable, extensible [Young01], [Wiegers03] and operable without creating any disruptions for the Users. The system shall be reliable and shall recover automatically from any system failure. The system shall be secure according to the Users mandate, usable by any designated users, as well by the assigned clients. The system shall be stable/ testable against potential issues.

\section{Requirements imposed by other participants}

Different Client's responsibilities and duties imposed and recommended by the LD/RD are linked with the Users SC system, making the system also dependant on their requirements. While using the system, the Users must comply with mandatory aspects required by $L D, R D$ recommendations; imposed by the SO's internal quality management and according to projects, and Clients; requested by IT corporate requirements from ITD (on hardware, software and networking technologies, management of applications, system infrastructure and control, monitoring, maintenance) and from BD (including management of operational requirements, system and business processes).

\subsection{URN Model}

The goal of the model includes the consideration of requirements related to regular operation of the system, maintenance, monitoring and refurbishment, rather than the analysis of functional requirements. It also shows interest, from the Users view, in the 
exchange of administrative information related to activities across the organization.

\subsubsection{Main Use Cases}

While designing the model, it has been assumed that the Users have knowledge of the system requirements and that they have been interpreted from the Users' own perspective. The Users have also knowledge of the mandatory requirements imposed by the LD, and required by their clients, while have limited information on other system and business aspects administered by ITD and BD. It has been also assumed that the BD would advise the Users on any potential updates or changes in the Users system or business aspects, and that such updates and changes will not be committed by BD and ITD without having approval from the UM and Stakeholders. We are modeling the Users system with consideration to the operational and organizational requirements that are set at the SO.

There are four categories of use cases related to the Users information system:

(a) Users work flow and activities: take a look at the way the Users work on the system.

(b) Administrative information propagation and report: presents how information is propagated in the system across SOs, through various notifications and approvals.

(c) First test then deploy: describes characteristics of the Users work, which is related to system maintenance, and might require additions to the system or handling of issues.

(d) System monitoring: is in a way a "wish list" of monitoring capabilities for the Users. Currently they have no access to monitoring services, system's logs, technical activities and services completed by the other department. We believe that these use cases have an impact on the Users work, due to their dependability on services provided by other SO, interference of projects, deadlines and objectives, while working in relations with available budgets and staffing. We hope that there is potential for improving the 
operational aspects of the Users activities, either by transferring some tasks, goals, contributions, responsibilities, services currently performed by ITD and BD to the Users SO, or allow the Users to manage their own system.

\subsubsection{A look at the working system's options}

The employees from the Users SO work on a system with two components: the 'network component' (NC) also known as the 'intranet', allows the users to exchange corporate information and work projects with users from their own SO and with other users across the organization; the 'specific component' (SC) known in other cases as the 'extranet', which allows the Users to process data records and information received from outside the SO's borders, from external clients. The ITD is managing the Users system, including applications, software and hardware components, while $\mathrm{BD}$ is managing the organizational and business aspects, including maintenance of business documentation, addressing of correction actions, administration of additions and of future IT work.

\section{a. Users activities and work on the system}

The work activities performed by the Users are given by their mandate; this requires constant collaboration, assistance to clients, regular acceptance of information from certain clients and processing of data records received from these clients, work with the LD to ensure that rules and legislations are being applied and respected accordingly, assistance and regular reporting to RD regarding the work with the Clients, reporting of system status and data warehouse to RD and UM, reporting to own management and stakeholders on projects status and progress, analysis of administrative information and data records. This type of work requires exchange of administrative information, 
notifications and requests for approval on many system layers, with different departments and managers. According to studies [Damian07] in this type of work activities, "the designers have less opportunity to seek out relevant knowledge because of the multiple layers of stakeholders", while the "project team members have a much higher chance of introducing miss-interpretations of stakeholder's needs at each communication level".

GRL graphs and strategies - The high-level GRL Graph from Figure 3.3 is presenting the main Actors with activities on the system, of interest for the Users work, and which contribute or have impact on the realization of the Users goals. We have already introduced some of the Actors, such as Users, UM, BD, ITD, LD and RD, Clients, Stakeholders. Other actors are Business Department Management (BDM) and IT Department Management (ITDM), which manage the respective department's own activities, overseeing approvals for projects, the distribution of staff on inter-departmental projects, and the deliverables schedule, the budgets and report on projects status, progress and objectives to Stakeholders.

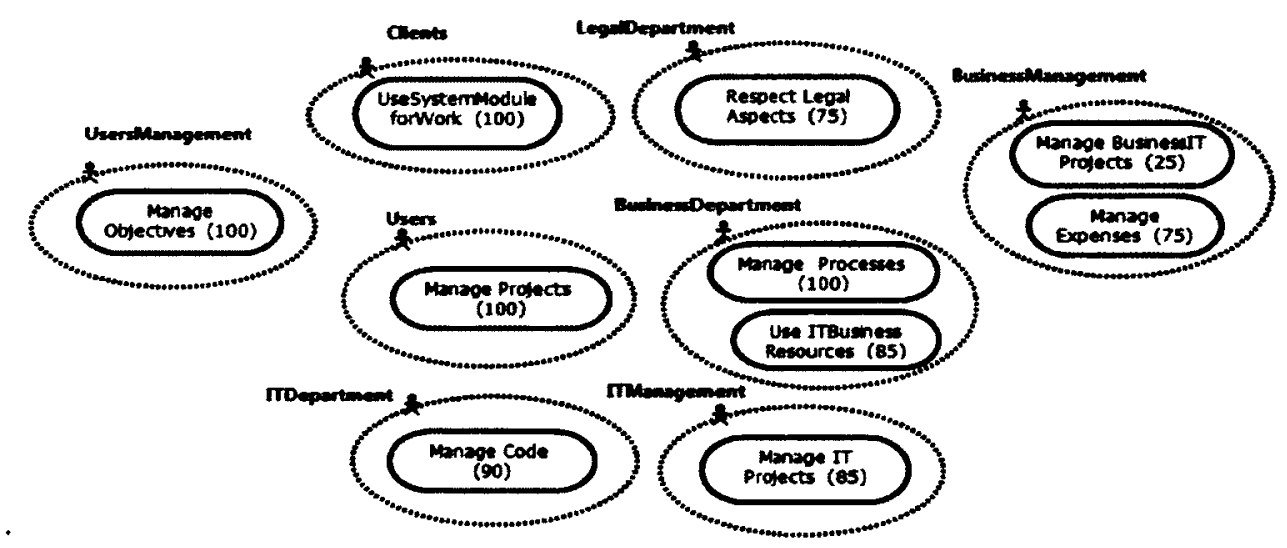

Figure 3.3: High level view of the system, presentig the Actors with few high-level goals

The Users often work with $\mathrm{LD}$, and on regular basis with RD. While LD provides guidance and legal advice for the organization's work, on legal issues, protection, law, 
partnership, and licensing, the RD ensures that rules and regulations are respected by any SO at any corporate level. LD and RD make sure that activities running across the enterprise are in compliance with most recent legislation. They also provide assistance in formulating legal documents, legal requirements, drafting and verifying documentation, and aligning documents and contracts with existing legislation and agreements. The GRL graph in Figure 3.4 presents the Actors with their Intentional Elements, indicating in details how each component contributes to the realization of other elements. The connections shown as lines with arrows between different components are the contributions of elements to the realization of other elements, including goals and Actors.

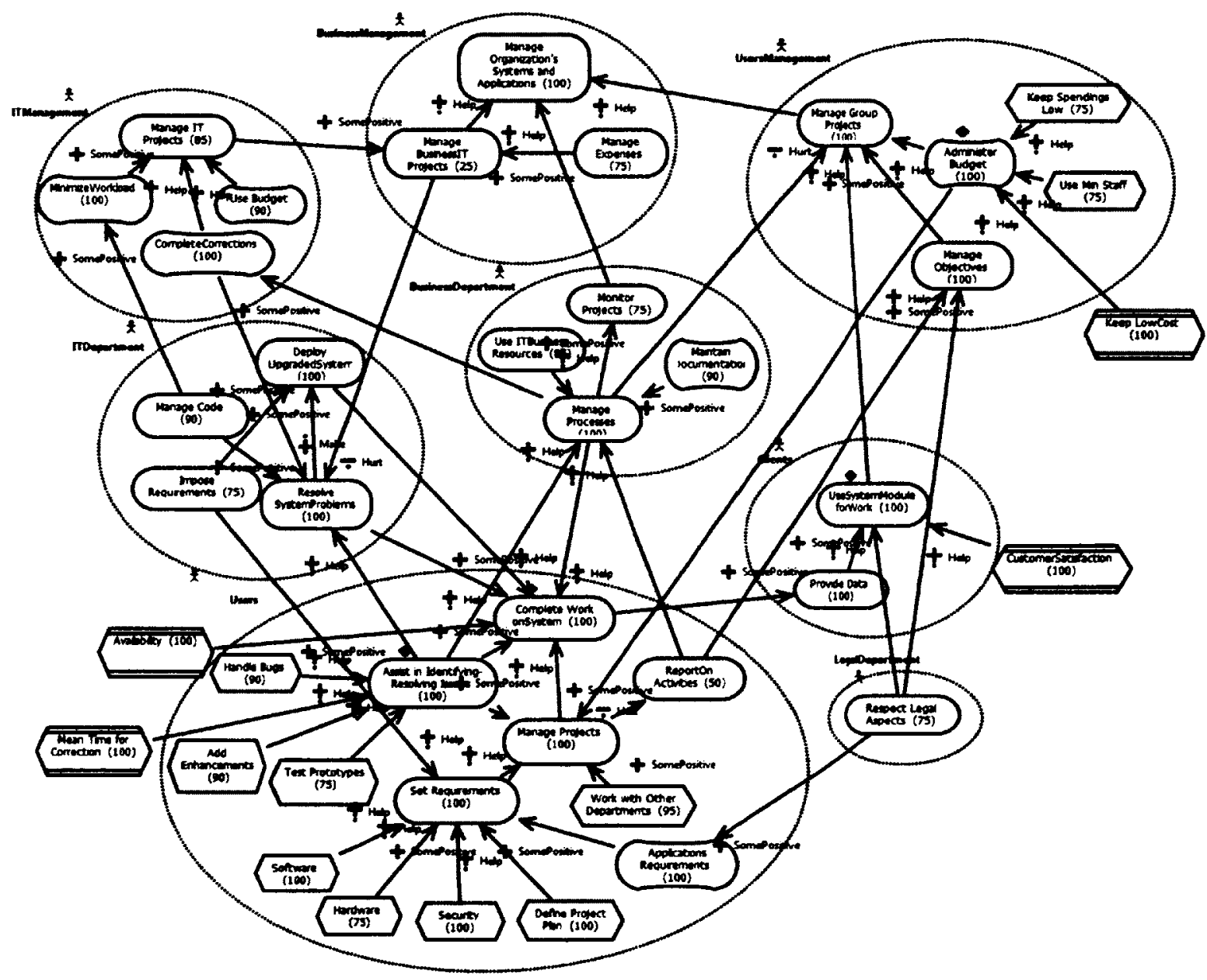

Figure 3.4: Detail representation of a GRL Graph 
For each Actor, the most important goals that contribute to the realization of Users goals and activities have been modeled from the system requirements, FR and N-FR. Some of the Users goals are indicated in Figure 3.4 as Assist in identifying-Resolving Issues, Report on Activities and Complete work on the System. Each goal contributes to the realization of the other elements/goals they are connected with. For instance, Report on Activities contributes to the realization of UM's goal Manage Objectives. Important goals from the other Actors are Manage Projects, another Users goal, obtained from the FR stating that the system shall accept to be accessed only by authorized users. Manage Code, Resolve System Problems (ITD goals) and Monitor Projects (BD goal) have been inspired from the activities performed by ITD and $\mathrm{BD}$, given that $\mathrm{UM}$ and the Stakeholders are approval authorities at SO/organization level for use of data and system applications. Set Requirements (Users goal), Respect Legal Aspects (LD goal) and Impose Requirements (ITD goal) have been also obtained from system requirements. Other softgoals and goals have been created from the N-FR, such as: Applications Requirements (Users softgoal), has been translated from the requirement indicating that the Users system shall be in compliance with rules and regulations; softgoal Maintain Documentation (for $\mathrm{BD}$ ) has been created from the N-FR that requires the system to have documentation maintained up to date according to specific procedures. Given that the Definition of Project Plans, Work with Other Department, Handling of bugs or Reporting on Activities can be done in different ways, by the ITD, as in the current system, or by an external contractor, if for example ITD staff is not always available, or by the Users, if some of the IT and $\mathrm{BD}$ responsibilities done for the Users could have been transferred back to the Users. Assist in Identifying-Resolving Issues and Complete work on the 
System, can be decomposed in components, such as Application Requirements, set Security, Set Requirements, with corresponding contributions. With the goals set, the evaluation and analysis of the graphs would determine how the goals will be satisfied for each different strategy at what satisfaction level and with what type of contribution from the other goals. The evaluation will also indicate if the system has a satisfactory design and will contribute in identifying incomplete and ambigously defined requirements [Amyot04]. Evaluation in early system development phases can provide the managers and stakeholders with valuable information prior to taking "high impact decisions very early in the development cycle" [Amyot03a]. "The concept of GRL strategies is a mechanism developed in jUCMNav to analyze GRL models using a set of user-defined evaluations" [jUCMNav 12]. For this case we present five strategies that will be explained in more detail in Chapter 5, when we analyze the results of the system evaluation.

UCM views and scenarios - UCM are used to decribe the system behaviour and structure at the architecture level, allowing for a flexible "allocation of responsibilities to architectural structures" [Amyot03a], which help in analyzing architectural choices at high level design, validating use cases and generating test scenarios. The scenarios "execute a UCM model" and present in a red highlight "the traversed path in the diagrams. One can then verify that the traversal matches with the intended behaviour and rectify the situation if this is not the case" [jUCMNav12]. The scenarios are based on the Users activities and on the interactions with the other Actors and their own activities.

Overview of scenarios - To perform their work, the Users have to log on to the system through a secure interface. Once connected, the access to applications and system functionalities are restricted to User's roles. The current implementation of the system is 
quite new, being recently refurbished from the old system due to outdated technology and applications. Different paths for reaching various Users responsibilities are presented in the scenarios summarized in Figure 3.5. The first scenario is a high level scenario presenting an overview of the Users activities as seen in Figure 3.5 and Figure 3.6. The second and third scenarios are allowing us to present details and alternatives on realizing the paths, depicting in a chronological order the responsibillities that must be completed along the path. For example, Scenario (2) from Figure 3.5 realizes the path that helps identify an issue during the Users work on the system, of type bug. Scenario (3) covers the alternative of identifying an issue of type addition, like missing or requiring additional functionalities.

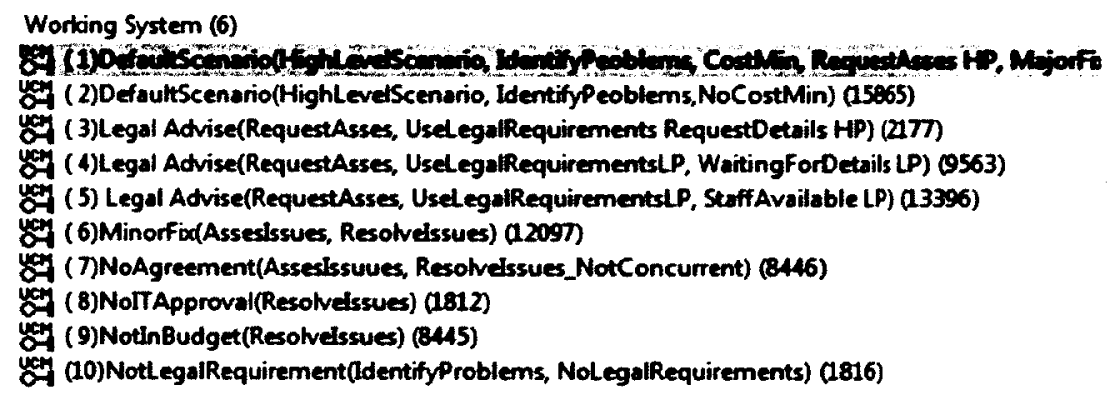

Figure 3.5: Summary of scenarios for User activities and work on the system

For the UCM described in Figure 3.7, Scenario (2) will execute the path directly, without waiting for details from the $\mathrm{LD}$, allowing the $\mathrm{BD}$ to asses the Users request. On the other hand, Scenario (3) indicates that details are required from LD, which sets the execution on waiting for a time necessary to $\mathrm{LD}$ to investigate the issue and get back to the $\mathrm{BD}$, in order to continue the execution of the process along the pathway. Now if the Users work requires the development and implementation of a new module or the clients require new functionalities, the Users address them first with the BD (see Fig. 3.5 and Fig.3.6). BD 
will further address the request with ITD, only after some verifications are done with positive results. In case of negative results, the request will be further discussed prior to taking any execution approach. In the use case maps, we illustrate these activities through stubs (see Figure 3.6). For example, the process of reporting issues is quite complex (Figure 3.6) giving that the Users have restricted access in administering the system. Anytime the Users have a request, they have to document it, report it it to the UM, advise it to $\mathrm{LD}$ or to the $\mathrm{RD}$ if necessary, and to Clients, if the request is related to their applications. Upon receiving resolution from the UM, and depending on the planned objectives, correction cost and available budget, the Users can then take the request to the next phase in the reporting process, and address it according to the case: for any requests related to the NC, the users must open a call by email with ITD Network Group (ITDNG). Any request related to the SC, network or production, must be addressed with the BD, which will further address it with the ITD Solutions Group (ITDSG). Communication with management from each group, UM, BM, ITM will be required often while working on requests. Any issues related to the development system hosted on the SC can be addressed directly with ITSG. The assesment of the request might require assistance from the LD before proceding with further investigation, while any issues found on the development system, hosted on the SC, can be addressed directly with ITSG. The process will continue as soon as the $\mathrm{BD}$ receives information from the $\mathrm{LD}$ (see Figure 3.9). BD will then rank the request (see Fig.3.10) and advise the ITD on future projects that require support from ITD. When the IT staff becames available, BD will formalize the process and provide them details. ITD will receive the request, analzye and assess the work to be done in their end; they might contact BD/users for details. 


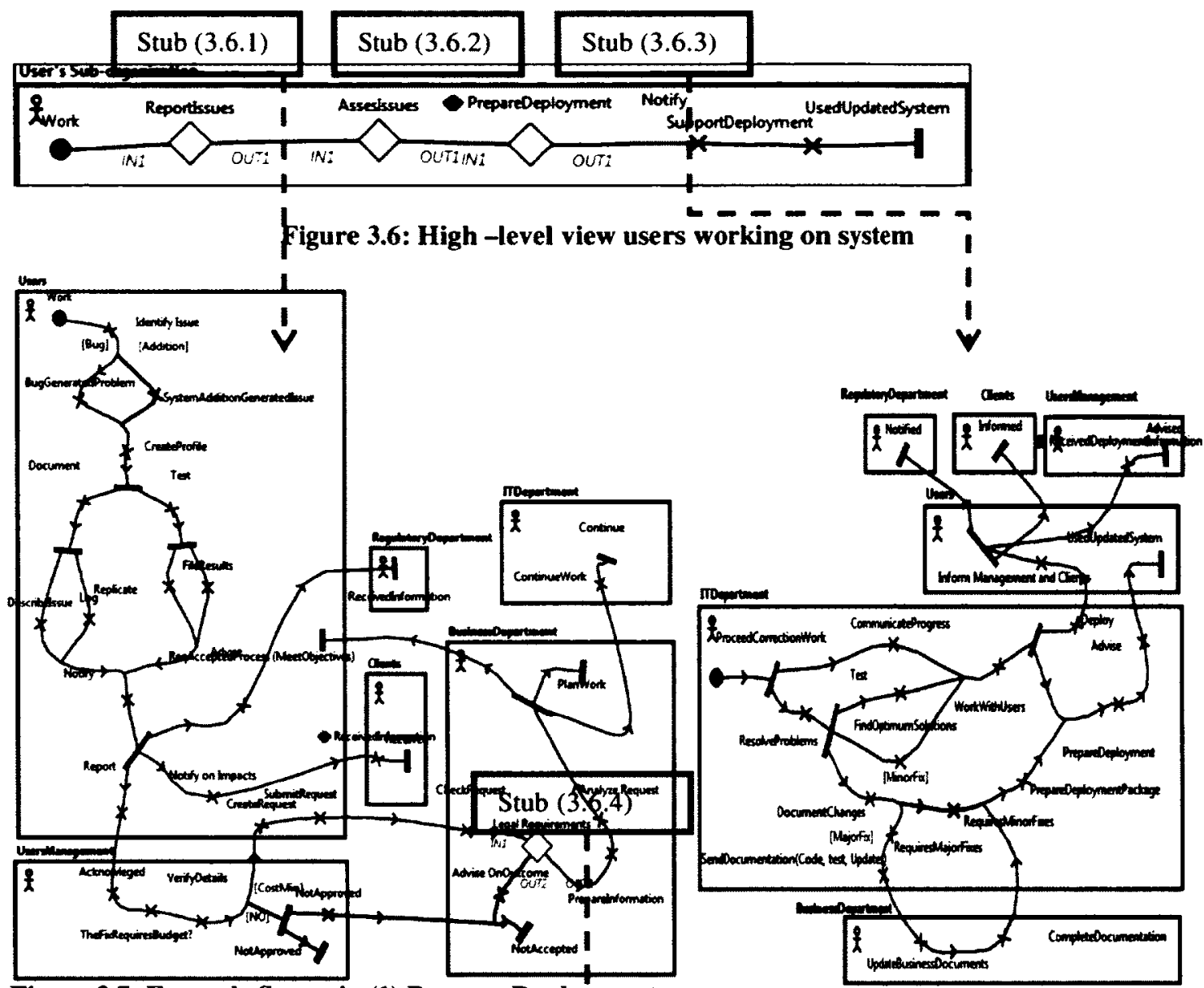

Figure 3.7: Example Scenario (1) Prepare Deployment (Stub 3.6.1)

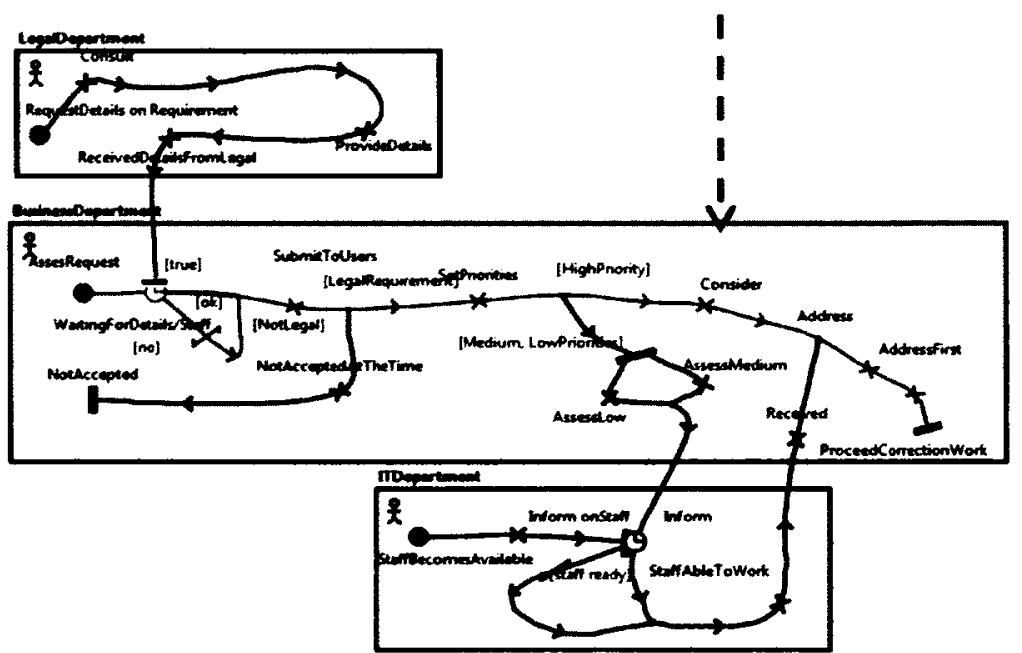

Figure 3.8: Example Scenario(1), Deploy Changes (Stub 3.6.3)

Figure 3.9: Example from Scenario (1) IdentifyIssues (ReportProblems) (Stub 3.6.4) 


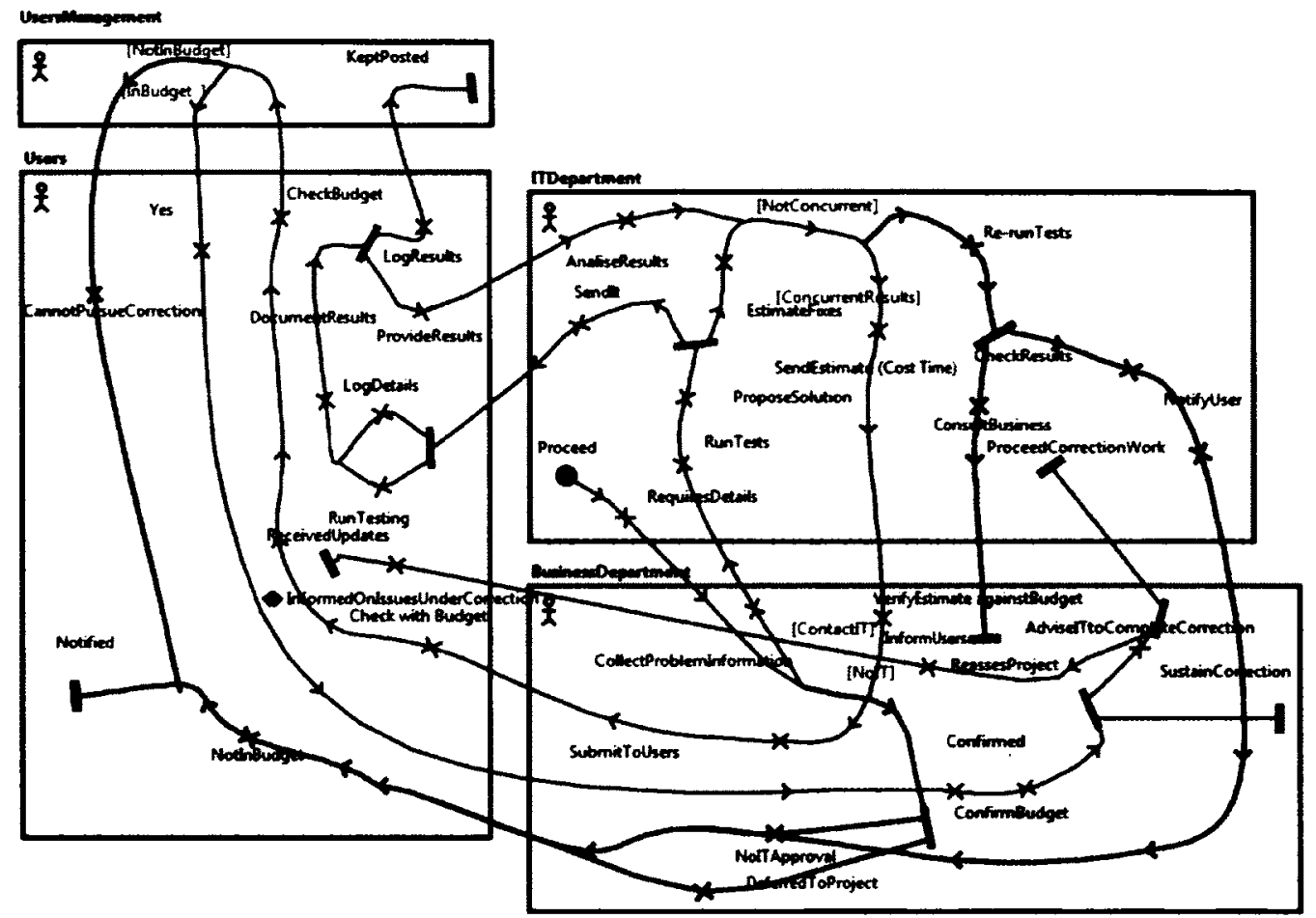

Figure 3.10: Example from Scenario (1) IdentifyIssues (ReportProblems) (Stub 3.6.2)

IT will run own tests and compare the results with the Users result.If the results are not concurrent with the user's feedback, and could require intensive work and higher costs, the ITD will inform BM and might defer the work for another project. If the results are concurent, ITD will determine any potential solutions solutions, estimate correction costs and timeframes for Deployment, and will send them for approval to BD. During the assesment process, the financial commitments required by the work are analyzed against the current budget. BD will send this information to the Users, which will further inform and contact UM for approval. If the process has been approved, the ITD could proceed with resolving the issue, designing the new application/addition to the system, by ensuring that associated responsibilities have been reached along the pathway, and that goals set in the GRL Graph have been met at a satisfactory level imposed by requirements. Once the assesment is completed and the request handled, we can proceed 
with the deployment of changes (Figure 3.6, Stub 3.6.3) process coordinated by the BD, ITD, with Users participation. Any time a scenario is executed, jUCMNav creates an

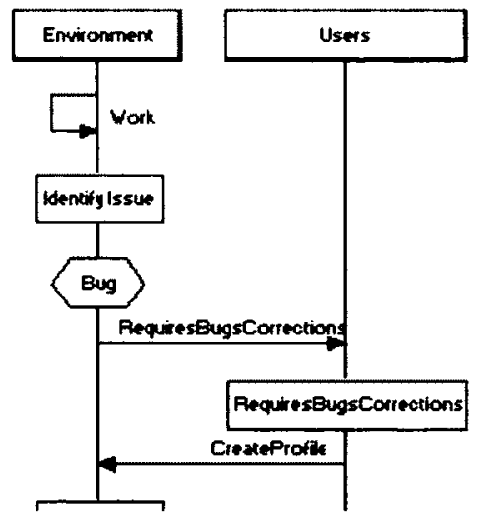

Figure 3.11: Extras from an MSC (some lifelines may not be shown) instance of the model; the results are collected in

a file and can be exported to generate a Message

Sequence Chart (MSC) or sequence diagrams in

UML [Kealey07]. The evaluation of the UCM

scenarios is based "on a traversal algorithm"

[Amyot07, Kealey07]. URN Links have been

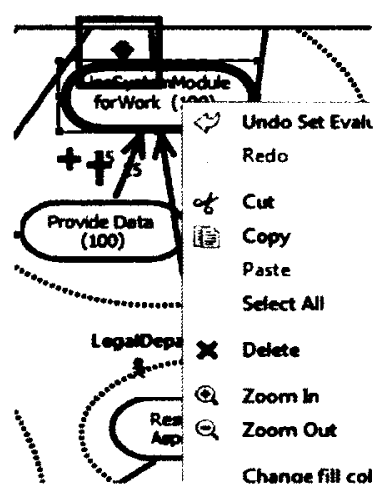

Chanae fill col

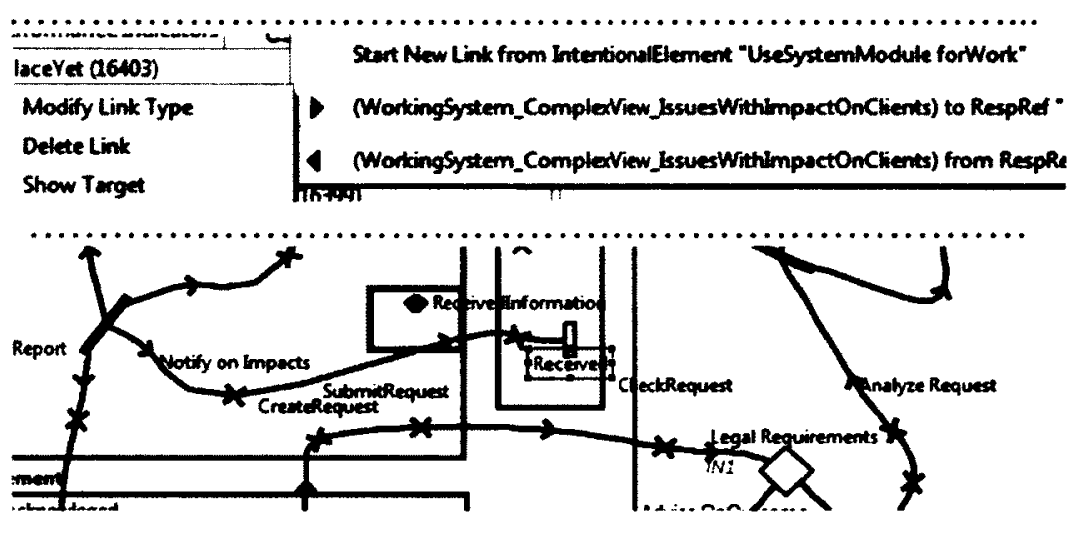

1........

Figure 3.12: Example of URN Links

and to measure the impact of changes on the linked elements [Roy07]. As presented in

Figure 3.12, the URN links notations are displayed as black triangle(s).

\section{b. Administrative Information propagation through the system}

In this case we study the way the administrative information related to Users activities is propagated through the system. This includes reporting of information to own management, Stakeholders, LD, RD and to clients; reporting on Users tasks, requests for 
approval and activities on data records and administrative and operational information; the identification and reporting of system problems/improvements to be added to the system; the periodical reporting of SO's managers to Stakeholders, on projects status and progress, financial statements, resources. The way the administrative information is being handled depends on the type of information, action requested, budget and available staff to process the request. The Users work with two types of information, the:

1. Administrative internal information refers to the information the Users work on and use inside their own SO. It contains: the information communicated/exchanged with/to colleagues, as required by projects; analysis reports provided by Users to UM, on quarterly basis and ad-hoc, as required; report studies prepared on data received from/and data provided to Clients; progress reports on projects and work activities at SO level; reports on system functionality, potential issues or additions for-seen, problems identified and resolved up to date. The administrative internal information also contains the information exchanged in inter-departmental projects with other SOs, data records analyzed as contributions to inter-departmental projects; the information exchanged with LD and RD at SO and organizational level; reports on system or application issues, addressed with BD and resolved with ITD, which include corrective action requests, requests for system maintenance, Users contribution to test cases, test plans, testing, comparison of testing results and work for deployment. It also refers to requests concerning budgeting, purchasing, staffing, contracting work, approval requests, notifications submitted to UM's, Stakeholders

2. Administrative external information refers to the information exchanged by the Users with clients and partners located outside their SO/organization. This information 
includes clients records (thousands of hundreds of records processed per year), data reports provided to $\mathrm{RD}$, clients and universities, at least few tens per year, data records provided per request to clients from the general public and to other organizations, approximate 4000 requests processed per year; data provided for inter-comparison studies conducted by other organizations, or for reports/year and national studies.

GRL graphs and strategies - With intentional elements, characteristics and contributors we constructed the model for this case (Figure 3.13). The goals and the KPI that have been selected, contribute to the Users work and to the realization of the Users goals.

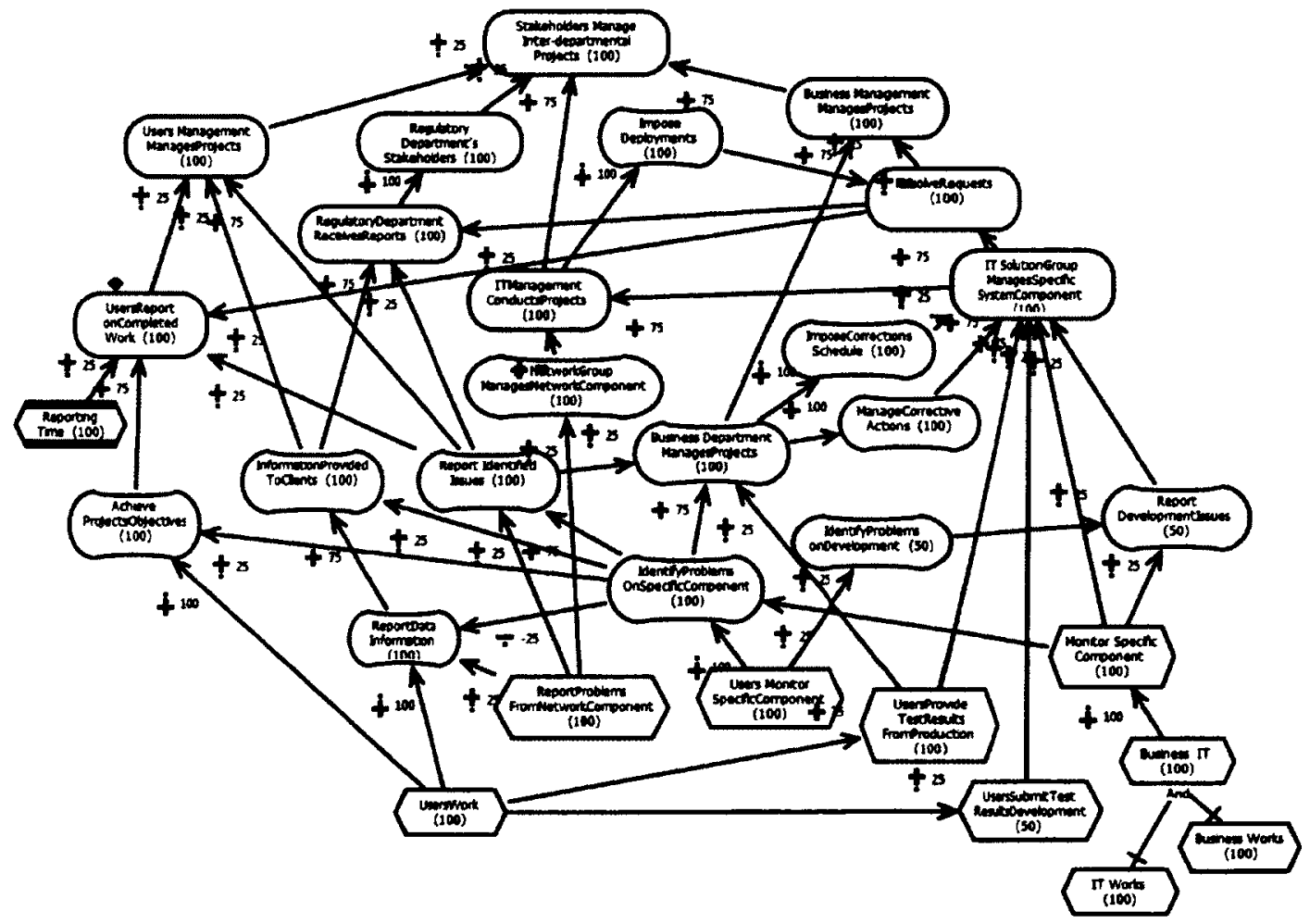

Figure 3.13: Administrative information propagation across the system

As presented in FIG BELOW, the exchange of administrative information is initiated during the inter-departmental processes in which each SO contributes with activities 
illustrated through Users Work, IT Works and BD Works tasks, which will contribute to the realization of many goals located above them. The users work on the system doesn't imply that they have monitoring capabilities, it depends on the strategy executed; while, when is true that if ITD work, it is also true that ITD monitors the system.

UCM views and scenarios - Different paths for reaching responsibilities are presented with six Scenarios in Figure 3.14. Scenario (1) is showing a high-level map of activities. The activities are related to Users work, The scenario presents how the requests with different priorities are being handled, using stubs to describe processes such as Manage

WortingSystem (6)
(1)WorkingSystem_CorrectionsRequests-HighWediumPriority (16356)
(2)WorkingSystem_CorrectionsRequests_LowPriority_DeferedToProject,
(3)WorkingSystem_WorkWithBusiness_CorrectBugs, Aditions (16359)
(1)WortingSystem_WorkWithBusiness_NoBusinessPlan_Doesn'tRequire
(5)WorkingSystem_ProductionlssuesHandled (6921)

Figure 3.14 - Information propagation scenario summary
CARs and Resolve CARs.

Scenario (1) presents a high-level activity map, while indicating the responsibilities involved in the information reporting process, as

shown in Figure 3.15. The use of Stub Work on System allows us to drill down in the model, have access to a new map described in Figure 3.16 and understand the responsibilities that exist at a new level, and have a detailed view of the Users activities from this view. The second diagram is enabling us to have a look at how the Users work and exchange administrative information from three network segments: the NC of the system, or the intranet, is used by users from all SO to exchange corporate data, store information; the $\mathrm{SC}$ of the system, or the extranet, is our case study and has restricted access to Users, some RD and ITD employees; the development environment of the SC, 
is also restricted to Users work, and is used by the Users to test different system functionality, while analyzing potential problems or during the testing process.

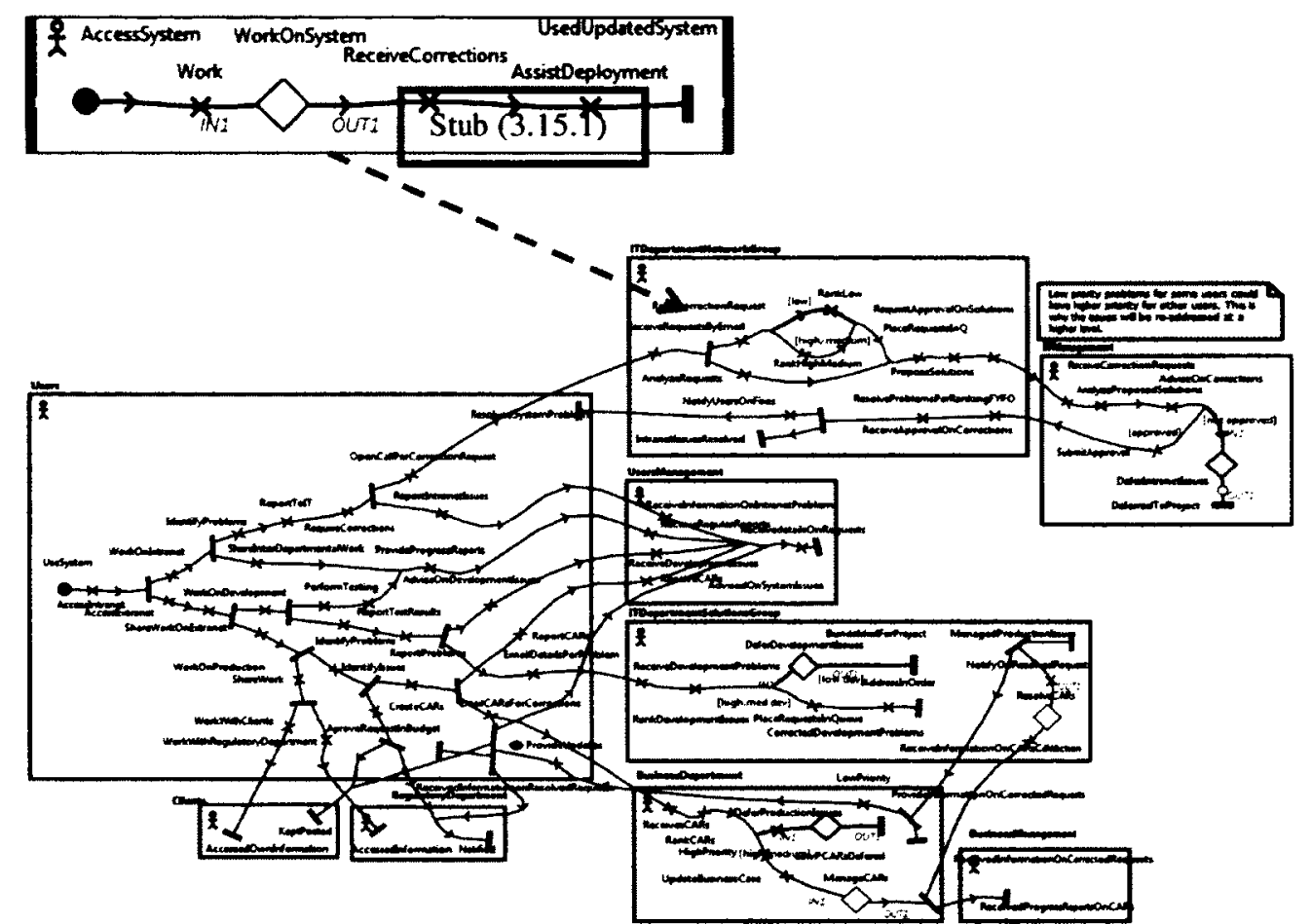

Figure 3.15: Overview of information propagation through the system (Users view)

To have a better look at at the map opened by the first stub, we decided to increase the size of it, split it in two halfs and work with the new maps obtained in Figure 3.16, and 3.17. Any issue the Users find on the NC, must be addessed by email with ITDNG. The IT will acnowledge the request, rank it and place it in a waiting queue to be processed. Any issue of concern for the $\mathrm{SC}$ must be addressed with $\mathrm{BD}$ by email as well. BD will acknowledge the request, rank it, analyze it against existing operational and project plans, then will estimate a finan-cial fee, if required, for the resolving of the issue. Based on budget and project plans verifications, the request could be accepted by $\mathrm{BD}$, and further processed, or it could be deferred to projects to be completed at a later date, or not accepted at current time. A notification will be send by BD to Users, ITD, BDM with 


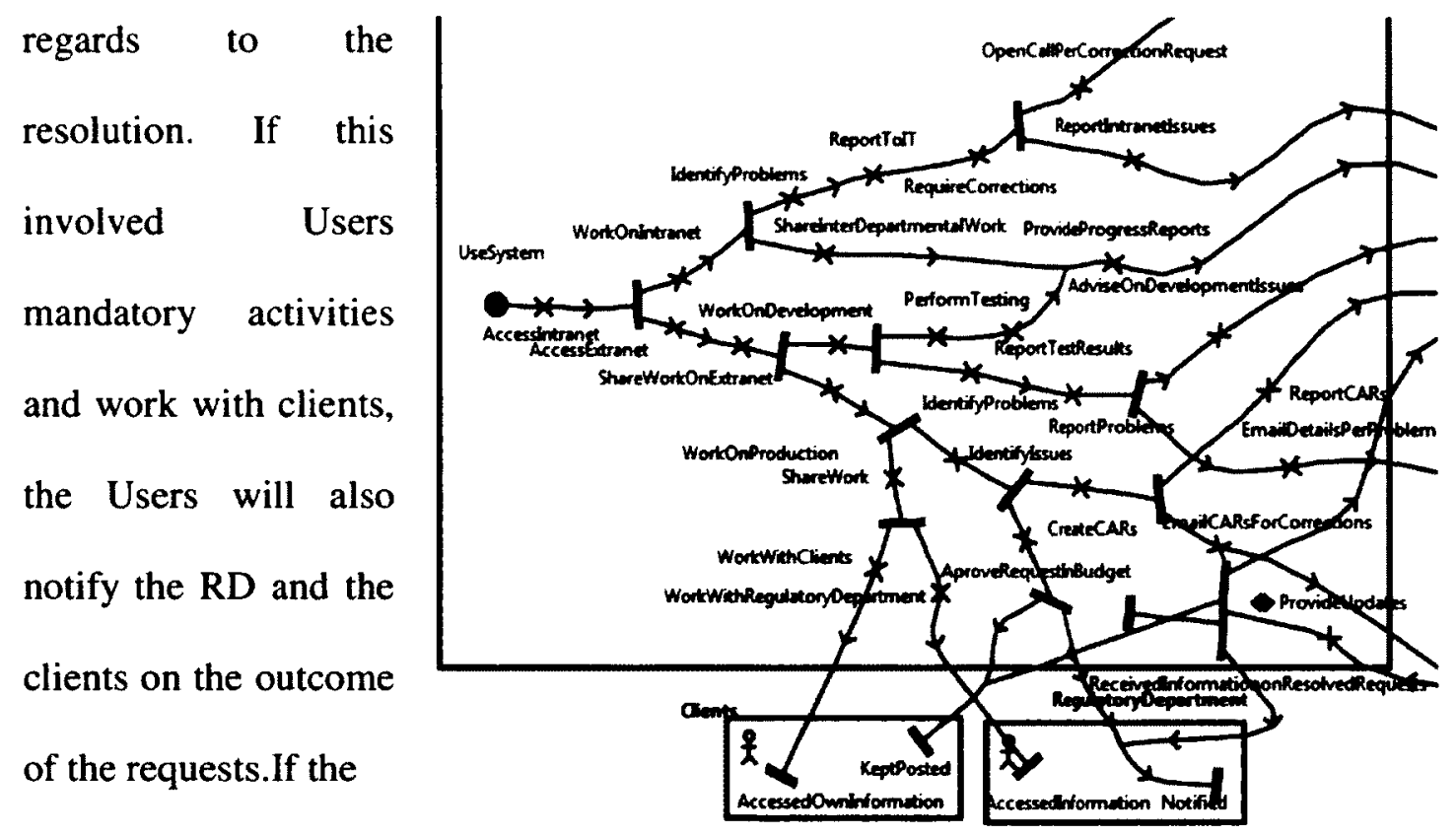

Figure 3.16: Extract from detailed look into the reporting information use case (view of the left half of the model)

request has been accepted by $\mathrm{BD}$, then $\mathrm{BD}$ will further report the request to the ITD. The ITD will acknowledge it, rank it in its own system, and place it in a waiting queue. The high priority requests received from all SOs will be analyzed and addressed in the order they were received. Depending on project plans and commitments, some medium priority could be also accepted, while the low priority requests are bundled up to be addressd at a later date, in the year, or will be defered to future projects for the next fiscal year. Any request submited by the Users to $\mathrm{BD}$ or ITD must be documented, and registered as a corrective action. The exchange of administrative and operational information between Users, ITD and BD also requires approvals from the UM, and consulations with RD and clients prior proceeding with decisions that might also affect the clients that are reporting data records in the Users system. The Users are also involved in the testing process prior any release on the Users system, therefore the testing information and testing results must 
be documented by participants and the information updated in the business documents, which falls under the responsibilities of the BD.By using stubs, such as Manage CARs (Stub 3.17.1), Resolve CARs (Stub 3.17.2), Defer Production Isues (Stub 3.17.3), Defer Development Issues (Stub 3.17.4), Defer Intranet Issues (Stub (3.17.5), we can present

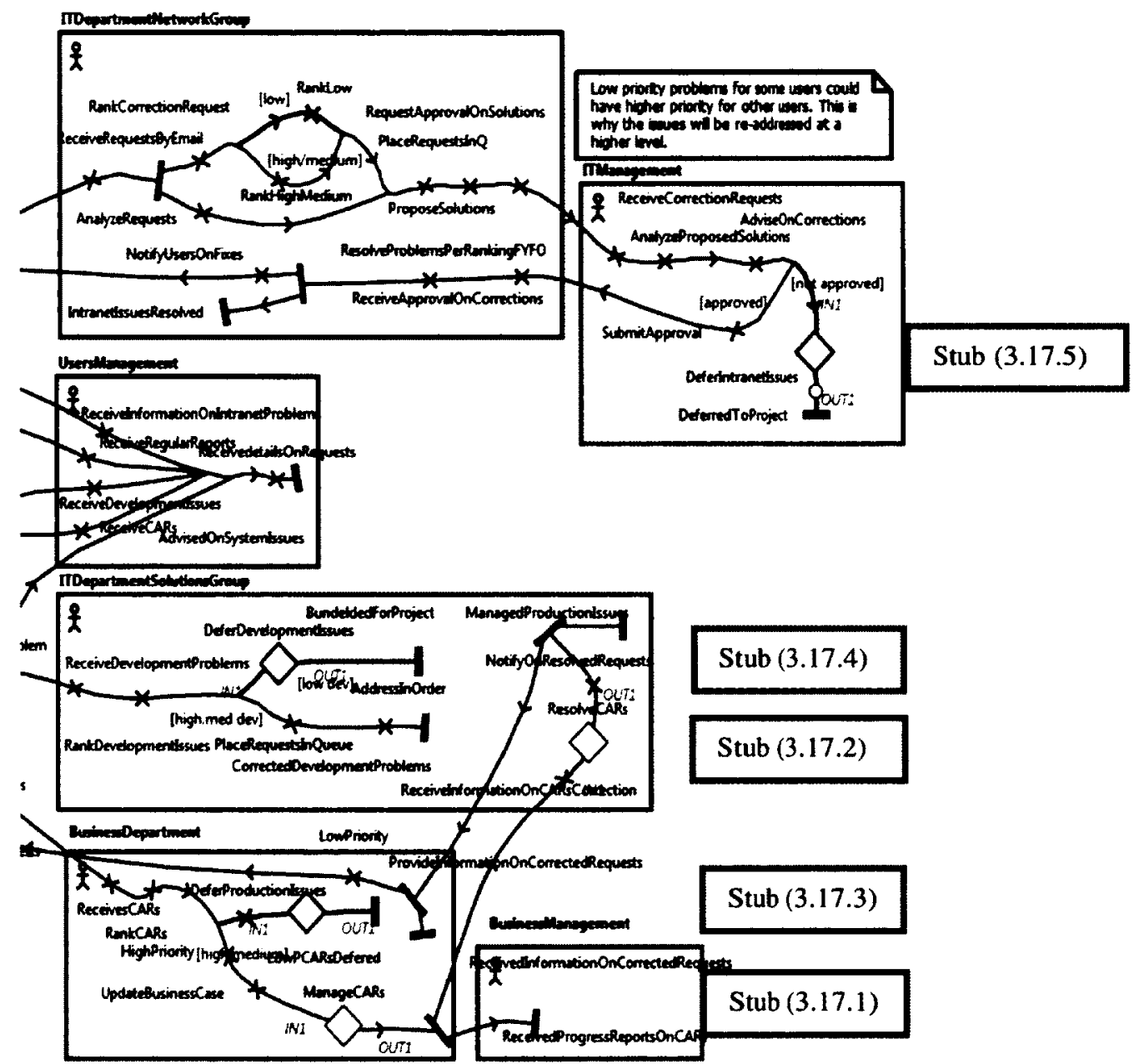

Figure 3.17: Extract from detailed look into the reporting information use case

more details in the model and add new path realization options. The next diagrams will present the connections between the parent map and submaps introduced by the stubs presented in Figure 3.17. To adress facts or issues identified on the SC, the Users have to create CARs and address them with BD, as indicated in Figure 3.18 (Stub 3.17.1, 3.18). 


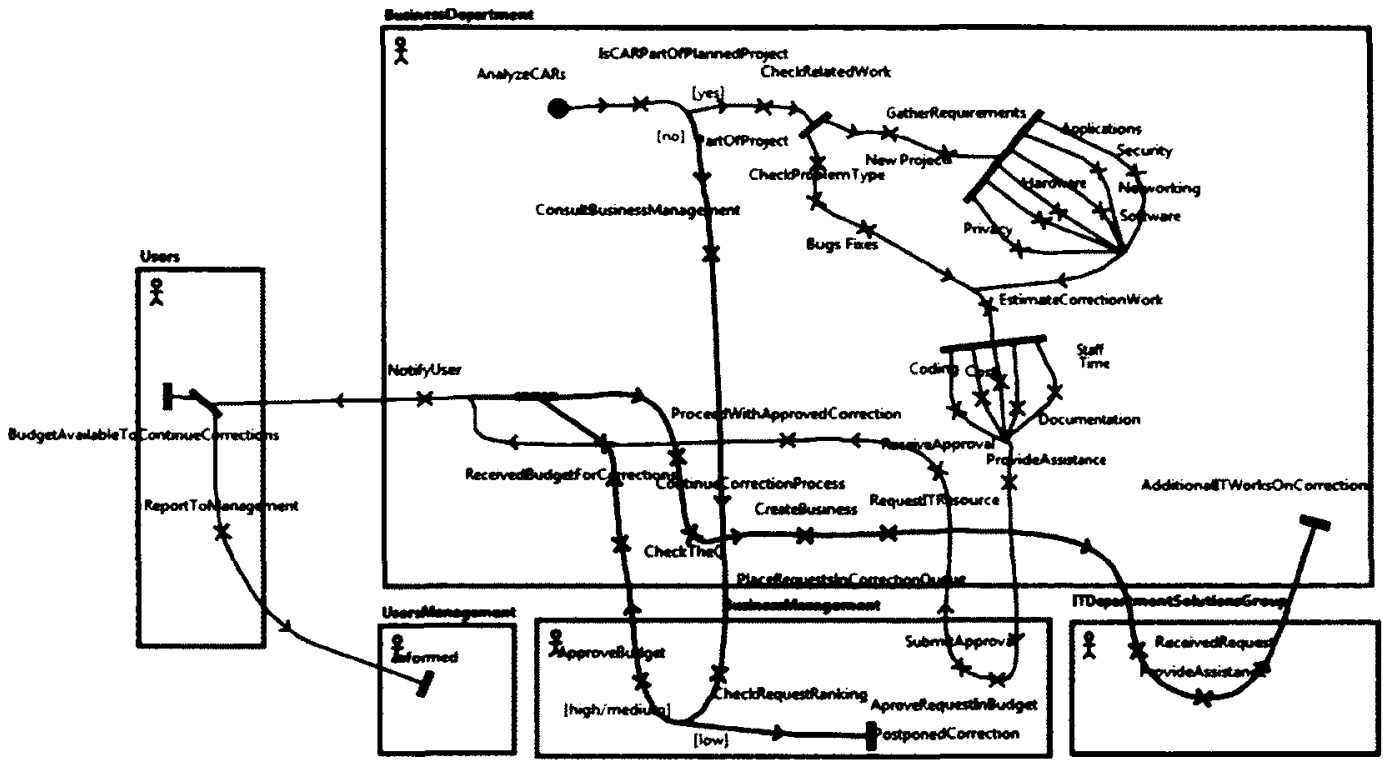

Figure 3.18: Detailed view of the complex work of the users (Stub 3.17.1)

If there are positive analysis of the CARs received by $\mathrm{BD}$, and managed through Stub 3.17.1, Figure 3.18 , the requests will be further submited for resolution to ITDSG, as indicated in Stub 3.17.2 with details on submap corresponding to this stub, Figure 3.19.

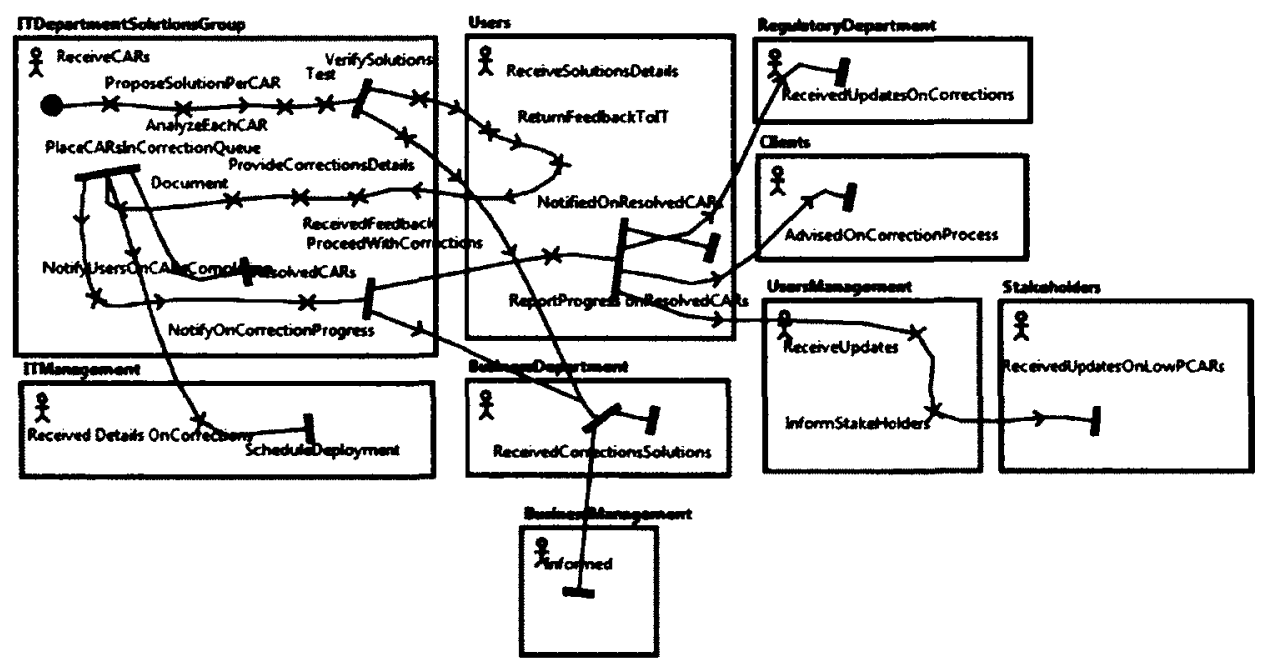

Figure 3.19: Detailed view - CARs/issues handled (Stub 3.17.2)

Else, for the case with CARs of low priority, or if there is no budget or staff to alocate to the correction process, the CARs could be defered as indicated in Stub 3.17.3 and Figure 3.20. In case there are facts or issues to be reported from the development environment of 
the SC, they are to be documented and addressed directly with ITDSG. If they are low priority or not related to the mandate, the requests could be defered to projects, as shown in Figure 3.21, Stub 3.17.4 and handled at a later date. In a similar way, the issues identified on the NG or intranet are to be documented and submitted by email to ITD

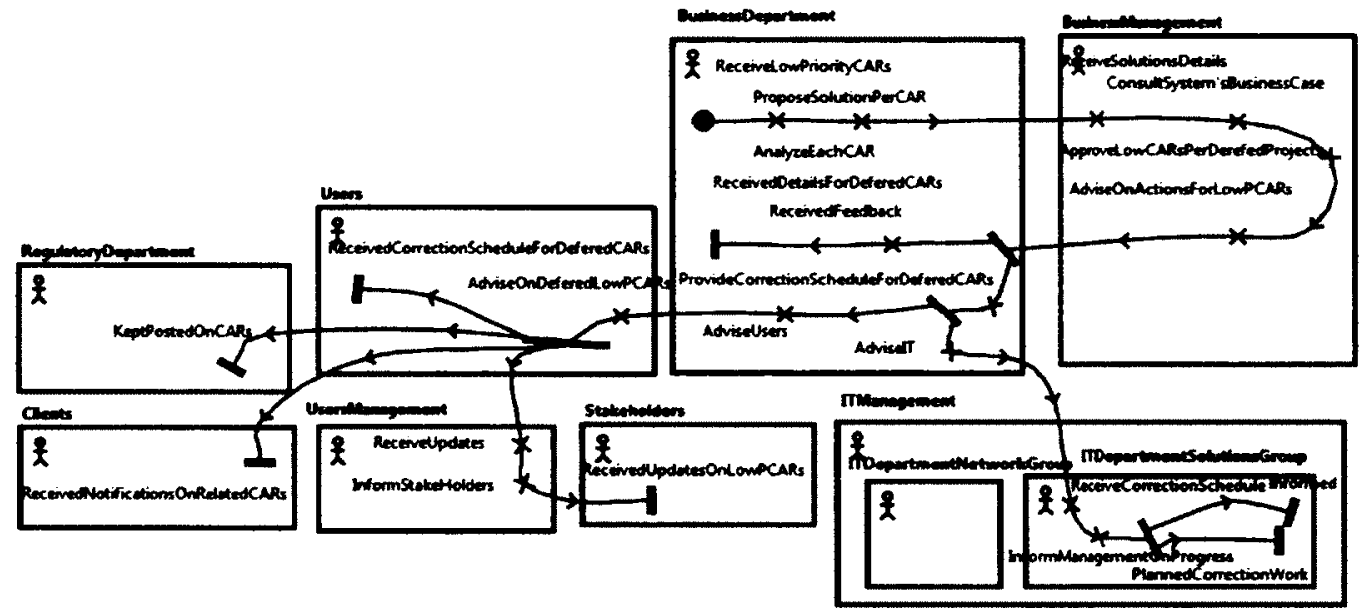

Figure 3.20: Detailed view, CARS deferred (Stub 3.17.3)

group ITDNG; they will asses the request and resolve them accordingly; low priority

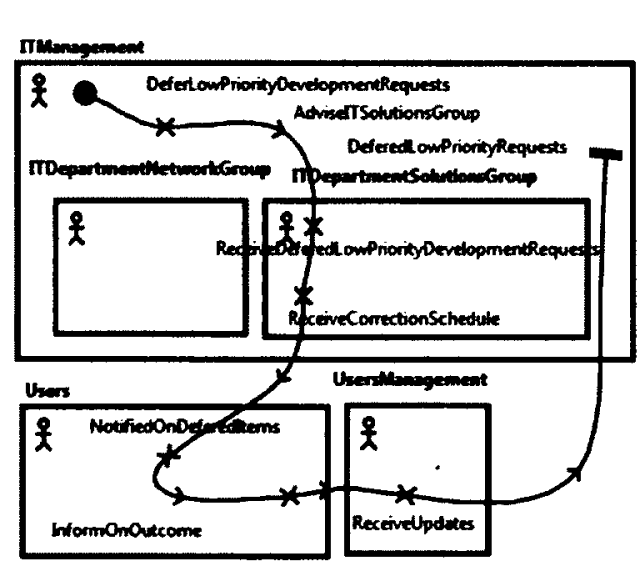

Figure 3.21: Defer to project low priority development requests (Stub 3.17.4)

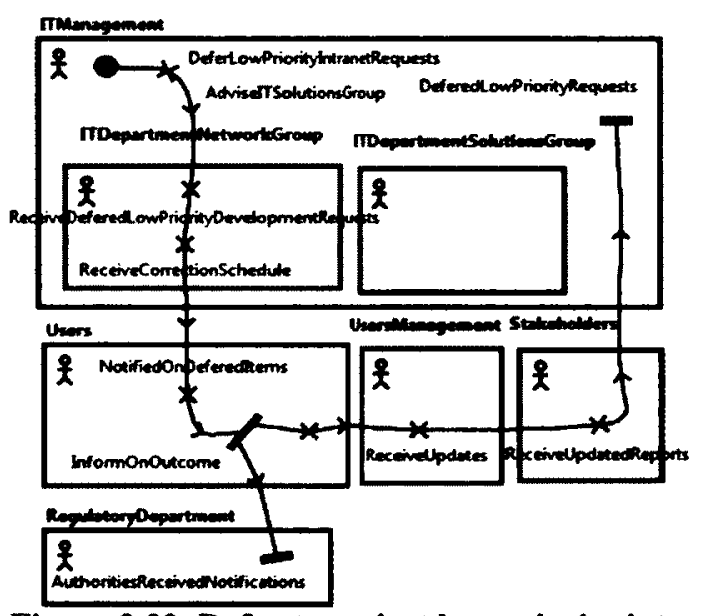

Figure 3.22: Defer to project low priority intranet issues (Stub 3.17.5)

requests could be defered to projects, as indicated in Figure 3.22, or proposed for more discussions in order to take the right approach and resolution. Scenario (2) for example is showing how low priority requests are handled, indicating details through stubs such as 
Defer Production Issues, Defer Development Issues and Defer Intranet Issue. Scenario

(3) is indicating the responsibilities that have to be achieved in order to have issues of type bugs or additions resolved. Scenario (4) walks us through a path that handles the case when this type of requests have not been previously included in the operational plan, yet there is budget available for their processing, and so on. We tried to name the scenarios in a sugestive and intuitive way. From Figure 3.17 and 3.18 we can see that numerous responsibilities are located with the $\mathrm{BD}$, such as administering modules of inter-departmental projects, planning of work for the clients, including administration of requests, management of corrective actions requests (CARs), ranking and organizing client's requests, assigning of staff, goals and deadlines to projects. The reporting information process to and from the other tens of SOs is lenghtly and requires approvals on many intermediate levels. Any update or change in the system, must be fully tested on both environments, development and production, by the ITD and by the Users, and their impacts on the system, including business rules, regulatory aspects and work with the clients, identified and reported to the Users SO for analysis and approval prior to proceeding with any further development. The documentation must be also kept up to date by BD while technical updates are to be maintained by the ITDSC. We believe the this type of information propagation, the reporting process and exchange of information activities introduces delays in handling and managing the request; requires correction costs, given the many services and department the Users must contact to have an issue resolved. All of this might have negative impact on the Users deliverables, if they are related to a certain issue pending corrections (e.g. if the server is down for a few days, the Users cannot provide information and data records to Clients). We believe that the 
transfer of some selected responsibilities related to Users system management, currently handled by BD and ITD, back to the Users, could contribute to reducing the handling and resolving of the issues and CARS; could reduce or eliminate the correction fees, delays caused by the way the information propagates; the amount of time, messages and acknolwdgments exchanged; could increase the Users knowledge in the system, and also help preserve the corporate knowledge in the Users SO.

\section{c. First test then deploy}

In order to address a request with the ITD, the Users must follow a reporting process established by the BD at organizational level, composed of the following steps:

- Document the request - Any time the Users need a change, addition, update or a fix to be completed on the system, they have to document it, present the rational and the description, advise if is related/imposed by legislation, indicate own ranking (high, medium, low) and advise on the impact will create on the system if not completed.

- Adress the request to UM for approval - Depeding on the feedback, the Users could submit the request to $\mathrm{BD}$ or post-poned it, if there are budget issues.

- Pending UM approval - Any rquest will be checked against operational plans/budget.

- Address the request with $B D$ - Upon receving approval from UM, the Users can email the request to designated business analyst from BD.

- BD acknowledges the request and places it in a waiting queue - based on Users ranking.The high priority requests will be addressed first, next medium and then low.

- $B D$ returns the request's tracking number - to the Users.

- Business analyst addresses high priority requests - BD evaluates each request with own ranking that will be communicated to the Users (they have limited knowledge on 
how the process runs and on managing requests placed in the waiting queue).

- $B D$ verifies the ranking of requests with the Users - If the ranking is acceptable, and there is Users budget available to support the change/correction, then BD will go ahead, cconfirm estimated currention cost and proceed with the request's investigation. If the request is not part of the operational plan and/or there is no budget to cover correction work expenses, then the request could be postponed or defered to a later project. If the ranking doesn't match with the Users ranking, further discussions will be required.

- $B D$ investigates the request - and documents it for work with ITD. Depeding on the feedback and on other circumstances, including Users operational plan, Users budget for the remaining period, BD and ITD own projects plans, budgets and staff availabilities, the resolving of the problem could be futther addressed with the ITD, or it could be assigned to a project that will be addressed at a later date, it could be postponed or rejected.

- $B D$ submits the request to ITD - the ones that have high priority, and budget available to support IT's correction work. If the request has been planned the year before in the operational planning, then will be addressed by ITD. If not, will be place in a queue waiting for an IT resource to become free.

- ITD addresses the request - Investigates and tries to recreate it on development. Then documents the results, proposes solutions, estimates correction price.

- ITD advises the Users and BD - on proposed solutions and cost.

- Users analyze the solutions and cost - If there is budget, and the solution and cost are available budget from other $\mathrm{SO}$ are used for own project, if acceptable.

- Users discuss solutions and costs with $U M$, requesting approval - to proceed with the solution recommended by IT. If approved, they will go ahead, else further discussions 
are to be set with BD, ITD, UM, BDM, ITM.

- Users confirm cost and solutions with ITD - and inform BD.

- ITDSG develops solutions, deploys changes on development - After they complete the testing, they will require the Users to test the corrections from their end too.

- ITD required Users testing on development - The User test the changes following on use cases provided by ITDSG, sometimes on test cases they developed

- Users complete development testing and document the results

- Users complete testing and send the results to ITDSG

- ITDSG verifies if there are converging test results - If test results don't match, then discussions will be required, else ITD will plan deployment schedule with BD and Users.

- ITDSG communicates deployment schedule to Users - looking to agree on the details of deployment; advises Users if assissance, support are required during the deployment.

- ITDSG deploys changes on production server.

- ITDSG completes testing on production to conclude deployment.

- ITDSG requires Users testing on production - to confirm the resolving of the issue, completion of deployment, and to close the process.

ITDSG closes correction process with $B D$ - As per the Users knowledge, any details of correction work and changes have been documented by ITD and BD, and included by BD in the system's operational and business documentation they administer

- $B D$ advises the Users upon closing the request.

- Users advise UM upon closing the request.

- Users advise RD in case the request had regulatory aspects.

- Users advise clients upon completion of request. 
Along these steps, the Users understood that many requests of acknowledgements and for approvals of actions have been also requestsed by BD to BDM, ITD to ITDM.

GRL graphs and strategies. Next we have modeled the way Users system changes are being handled, from the initiations as requests by the Users, up to testing and deployment by the ITD (Figures $3.23,3.24$ ) presenting the participant actors in the propagation process, their tasks, contributions, and related goals to be achieved. In Chapter 5 we will look at a set of 4 strategies, that are describing, with the use of four cases, how the requests for corrections or additions to the system are being handled, resolved, tested and deployed. Aside the general way of managing issues, the four cases investigate potential solutions for completing correction of issues/system maintenance work, are handled by:

- ITD with support from the $\mathrm{BD}$, as presented in the current situation

- a contractor, hired by the Users SO to help resolve issues /additions, or hired on contract by the ITD, in case the issues are urgent matters requiring correction

- Users, who are able to support responsibilities and activities managed by ITD and $\mathrm{BD}$, given that some have programmig background, and are knowlegeable of development and maintenance of software aplications, services and processes runing in the system

- purchase product, either off the shelf or developed specific per system's requirements. 


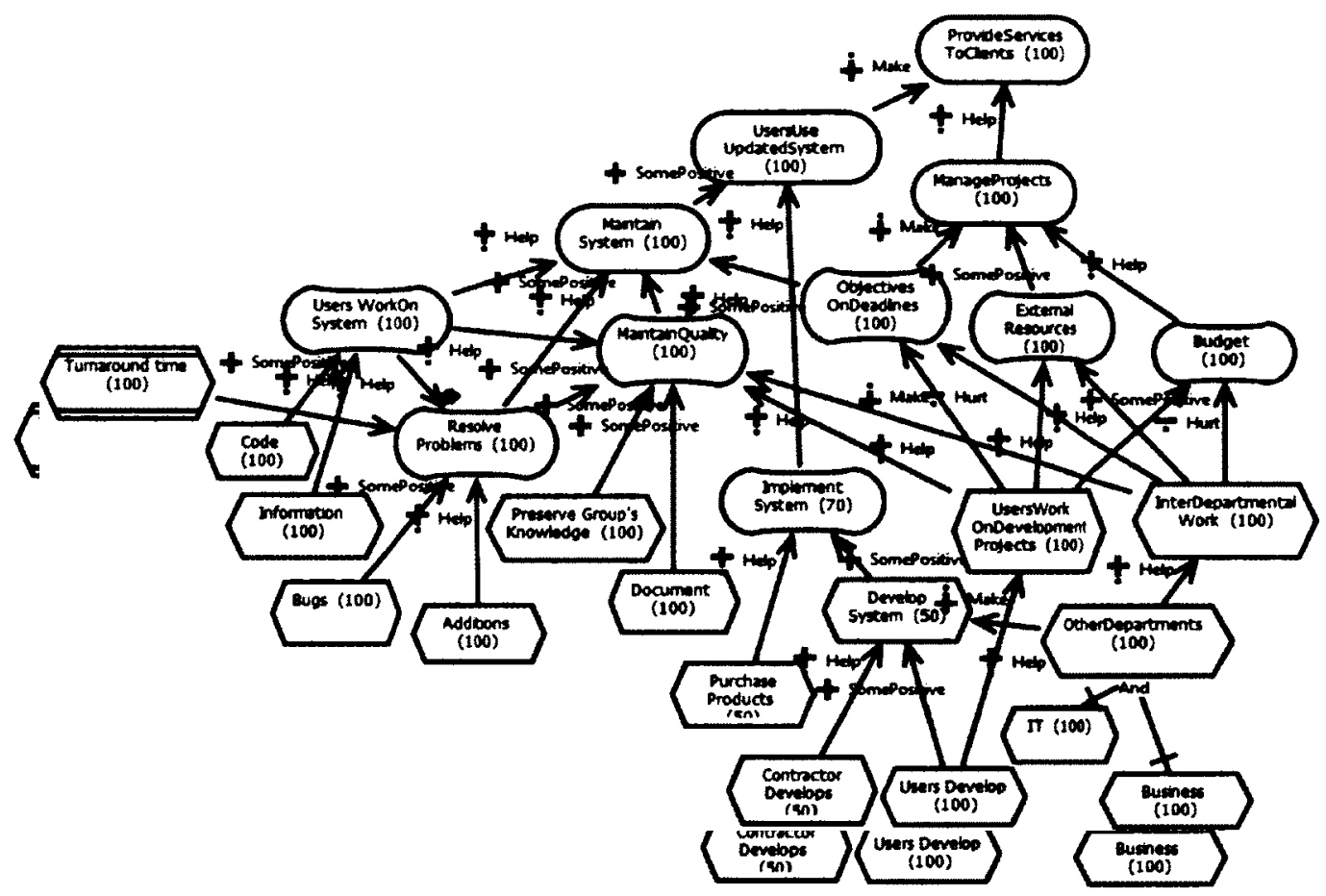

Figure 3.23: GRL graph presenting details for test and deploy case

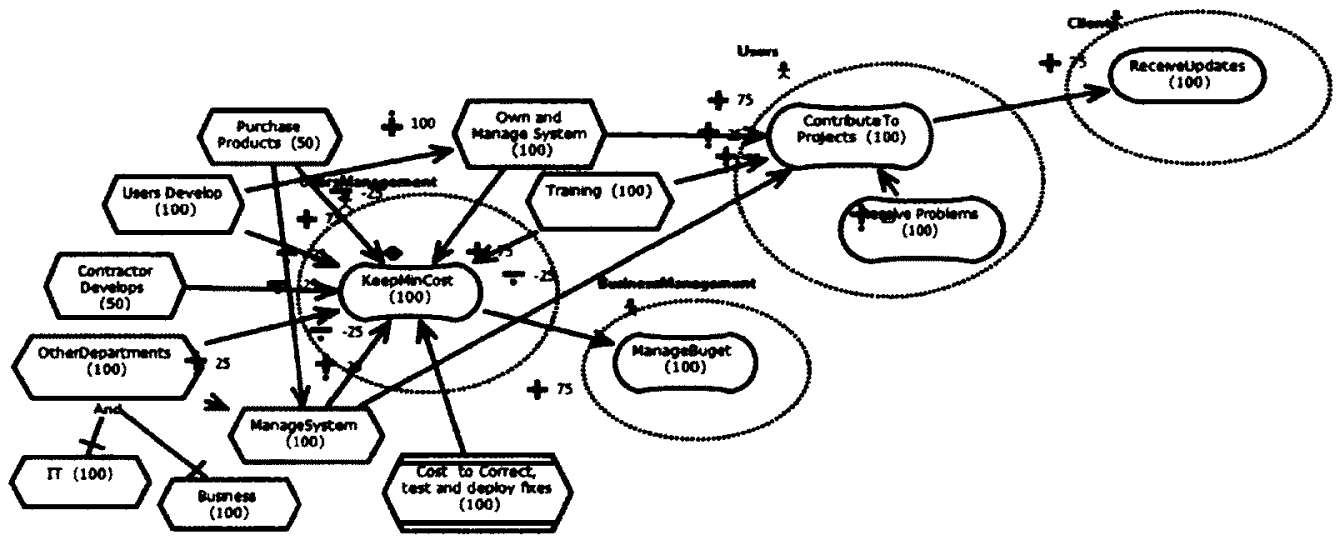

Figure 3.24: High-level view of case test and deploy 


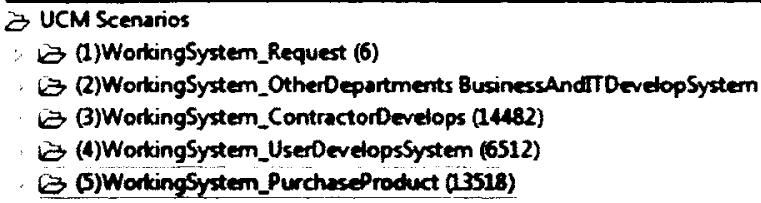

Figure 3.25: Scenarios summary, test and deploy

UCM Scenarios

$B$ (1)WorkingSystem_Request (6)

Yy (1).1)Defoultscenario_Request (774)

Yy (1.2)ProposalDenied, BudgetAvailable (B45)

- (1.3)NoBudgetAvailable (1233)

(2) (2)WorkingSystem_OtherDepartments BusinessAndT DevelopSystem

$B$ (3)WorkingSystem_ContractorDevelops (1482)

$\theta$ (4)WortingSystem_UserDevelopsSystem (6512)

(5)WorkingSystem_PurchseProduct 03518)

Figure 3.26: Scenarios details, test and deploy
As indicated in Figure 3.25 and

Figure 3.26 , there are five categories of scenarios: the first one is presenting a high level view map of the process, with three possible subscenarios, when budget is available or not, and requests can be accepted for processing at that time, while the next four scenarios

are showing in details the paths. To be able to work with data records and provide information and assistance to clients, the Users must connect to the system. The system is quite new, therefore is expected the possibility that Users or the Clients would require other additions, giving that the new technology and software could support more functionalities. To proceed with such request and innitiate a project, the user must request approval to UM. The request could be accepted or defered to next projects, depending if is part of the operational plan for the year and if there is budget available for it. The dynamic stub indicated in Figure 3.27 allows us to drill to the next layer of activities and access the responsibilities the participants have, according to the type of request. This will allow us to analyze the processes that are to be completed and evaluate how, the system will be maintained, depending on the development method. As illustrated below in Figure 3.28, the request can be approved, deferred to other projects or rejected, according to each of the four cases proposed for analysis and evaluation. The IT is in charge at the organization level with system and applications management, including 
purchasing of equipment and software applications, development of applications, information systems, programs, also testing and deployment; while BD is in charge with

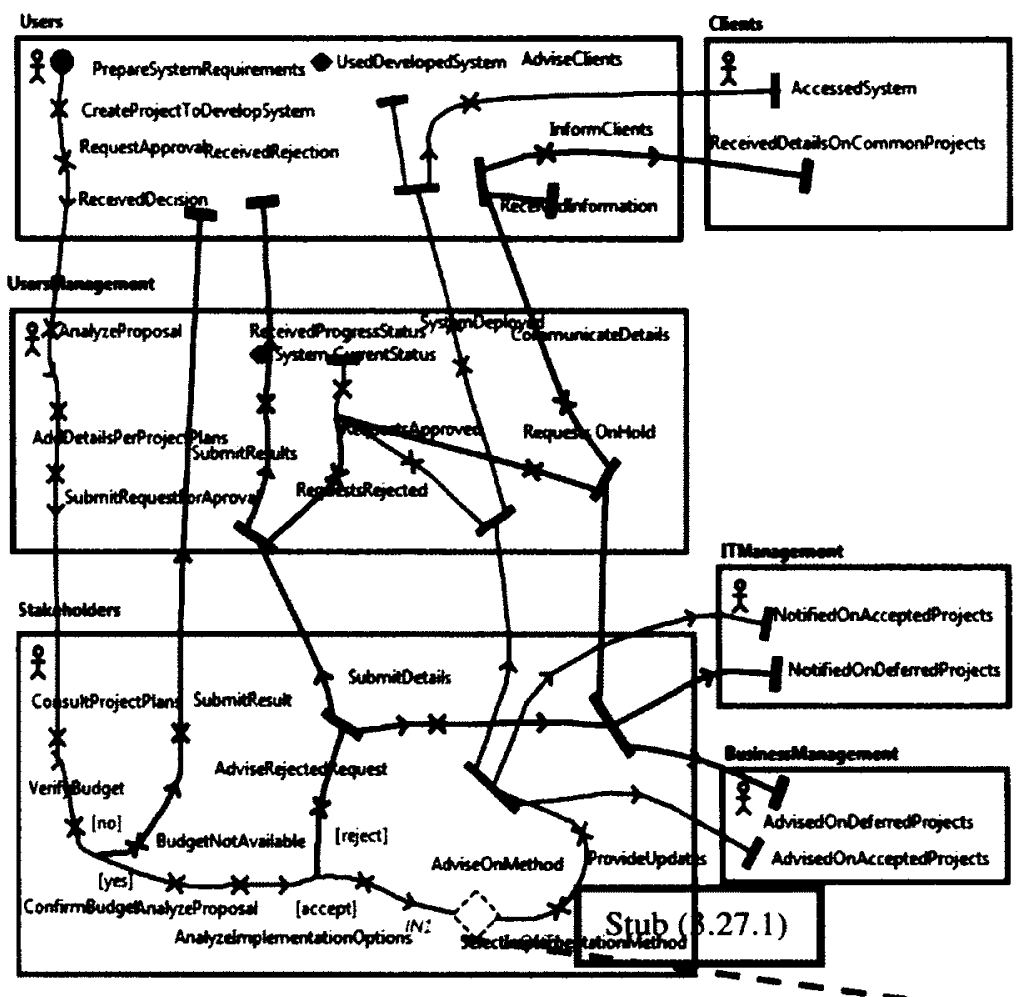

Figure 3.27: High-level view of test and deploy case the management of

business activities,

related documents,

activities workflows,

relationships, and the

contributions towards

achieving the final

goals are presented for

each of the following

four cases.

Setect plugin

YOthe-Departiments

YyH Contrectorowelops

Hiferdectops

Firehoseproduct

\section{c.1. System maintenance/development supported by Other Departments (ITD, BD)}

The correction and maintenance work of the system are managed by BD and ITD through

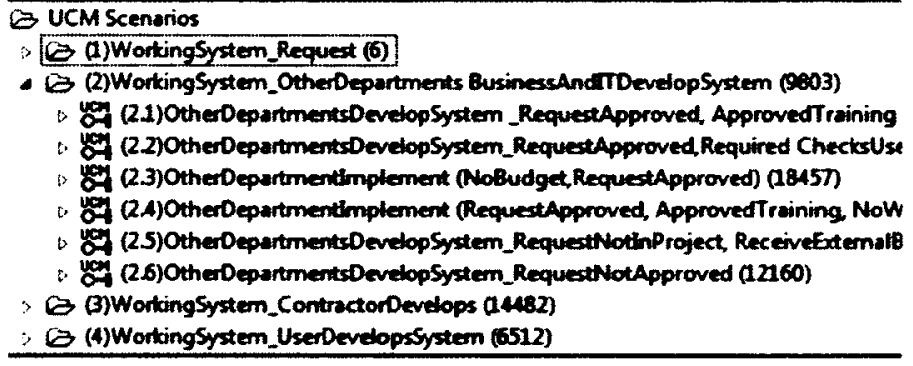

Figure 3.28 - Fig Extras from scenario list projects created across the

organization, in between

departments. The process is

presented with scenarios in

Figure 3.28 and UCMs 
Figure 3.29. Next we present the maps from Figure 3.31, Stub 3.29.2 corresponding to "Requesting approval for deployment", the process of requesting training Figure 3.32,

Stub 3.29.3.

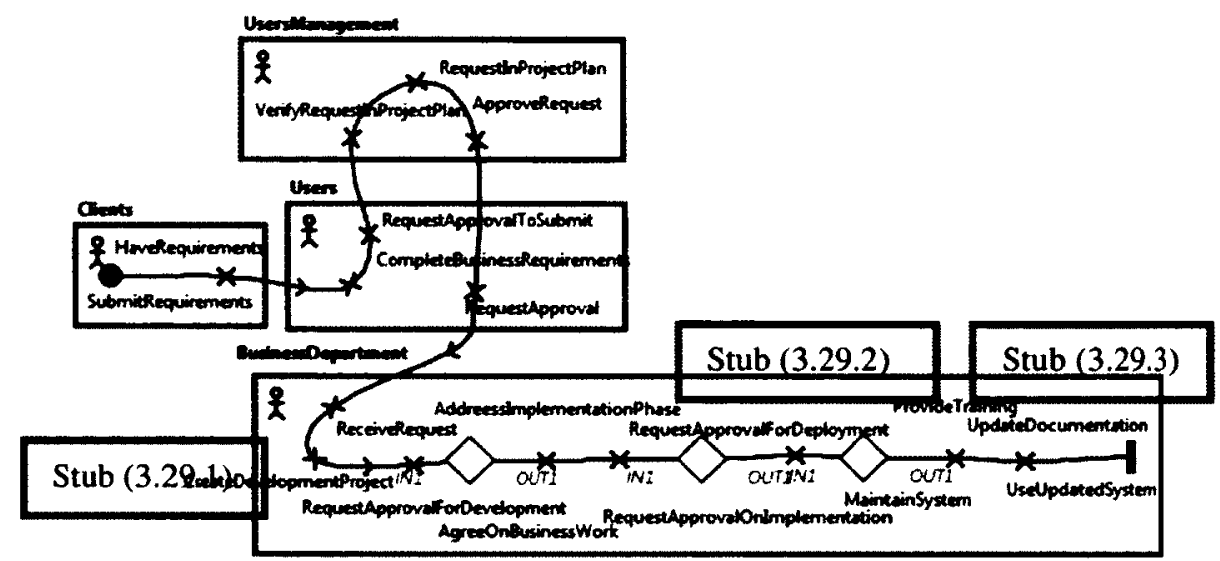

Figure 3.29: Sub-map business IT develops (Figure 3.27, Stub 3.27.1)

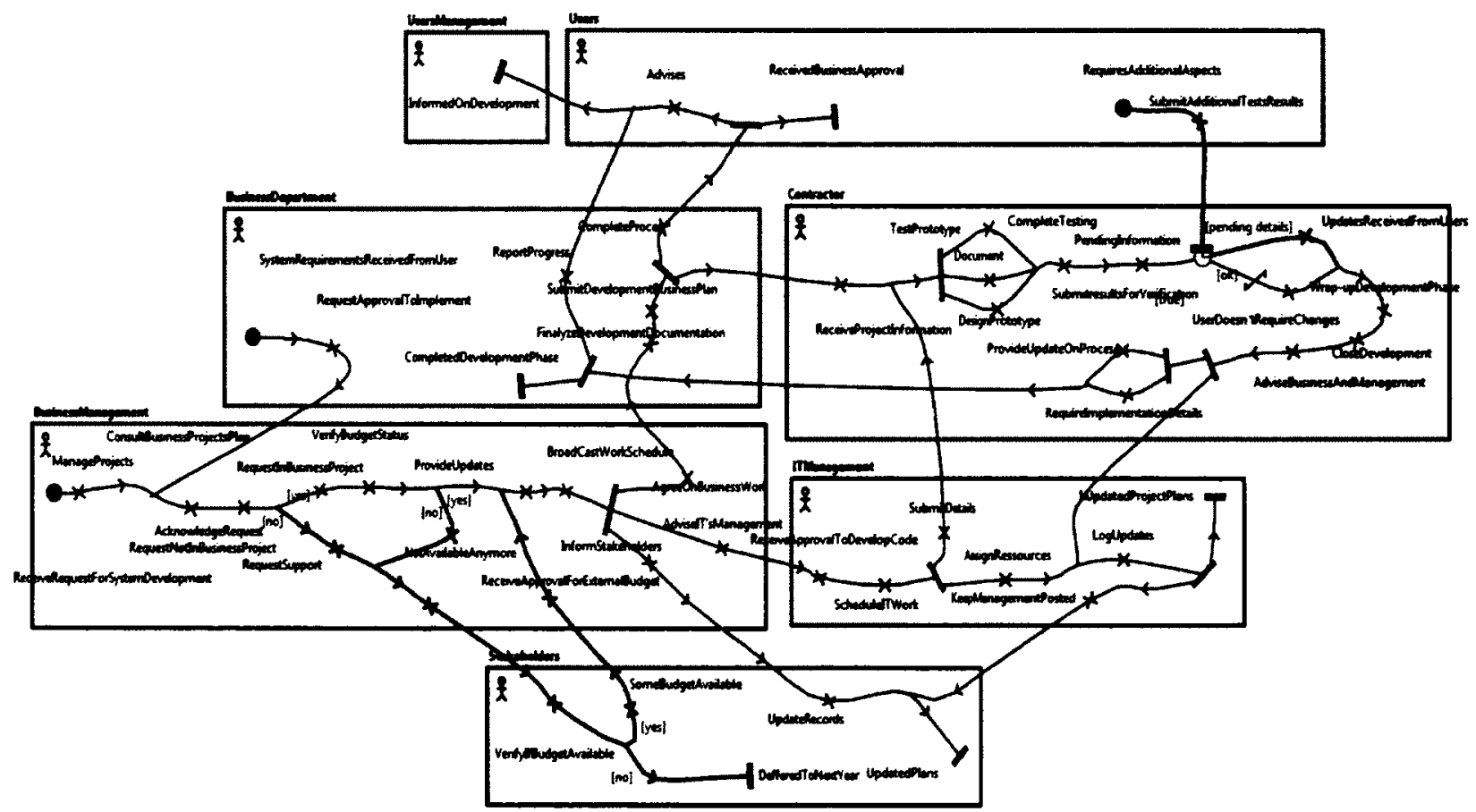

Figure 3.30: Overview of ReceiveRequest (Stub 3.29.1)

The areas that are not highlighted in red during the scenario execution have a condition or a state that will be reached in the other scenarios. 


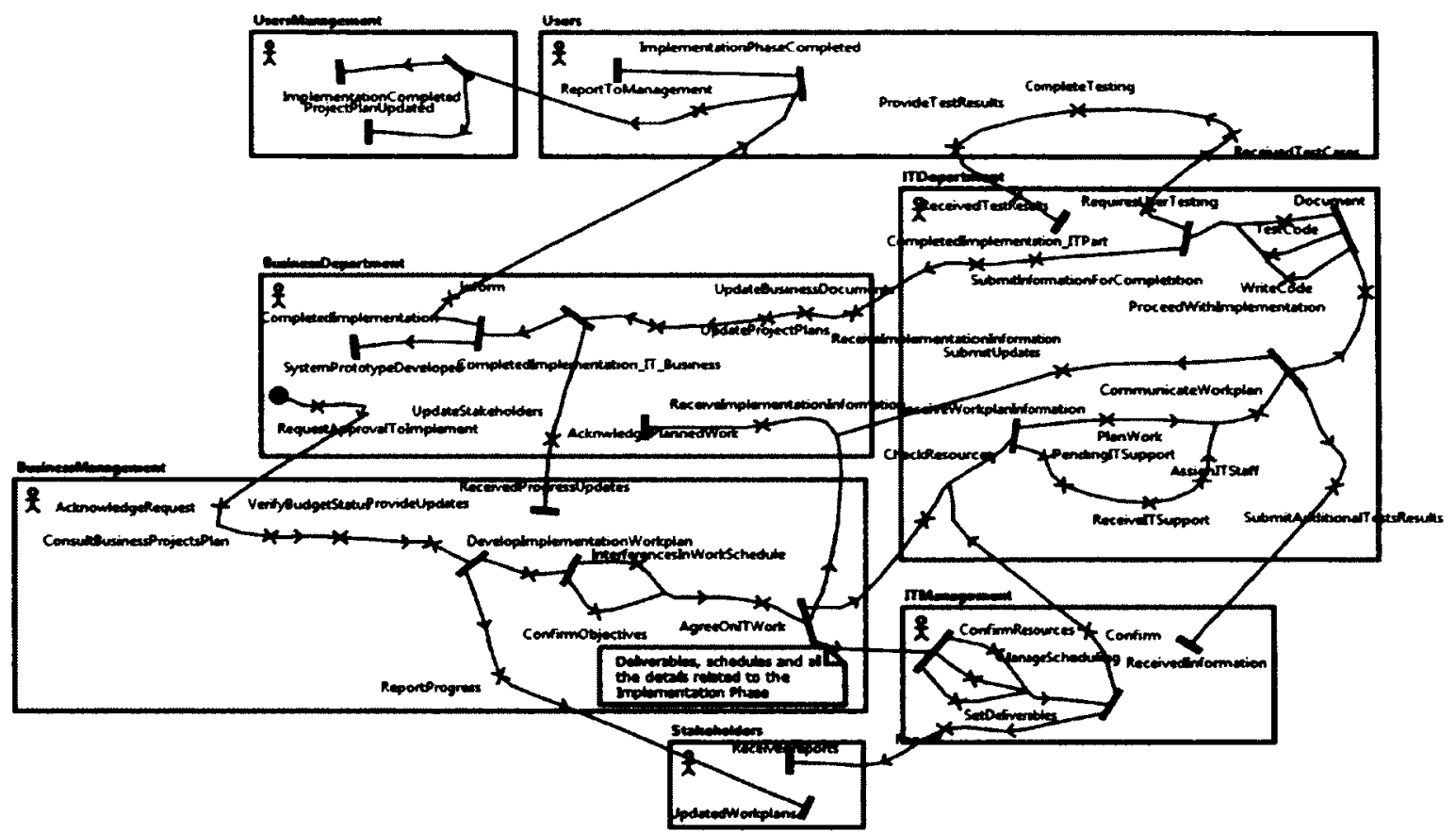

Figure 3.31: Details on Request ApprovalOn Implementation (plug-in for Stub 3.29.2)

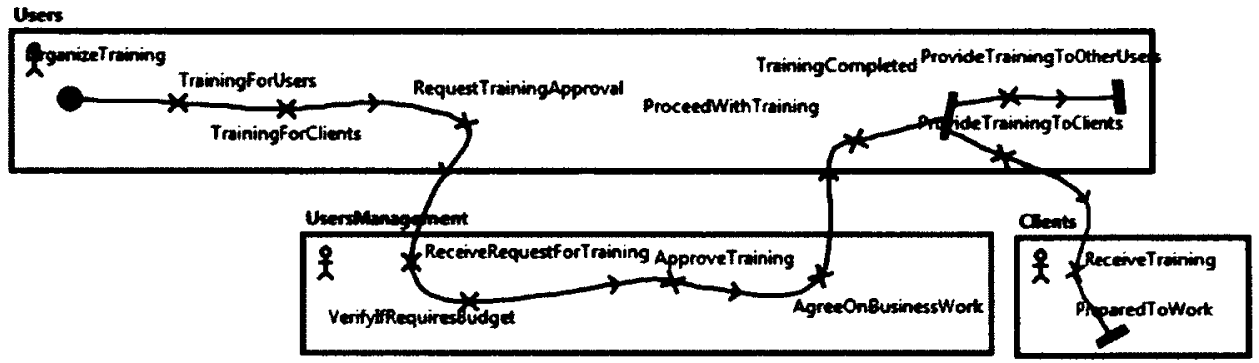

Figure 3.32 - Details on ProvideTraining Stub (3.29.3)

\section{c.2. System development supported by a Contractor}

This case presents a Contractor's contribution in developing system functionalities and resolving issues. The Contractor's work is paid from the Users budget and it roles according to contract's terms established according to Users work.

We work with two scenarios per Figure 3.34: in Scenario (3.1) the Contractor requires IT support, in Scenarion (3.2) it doesn't, still assistance will be available as per contract. 


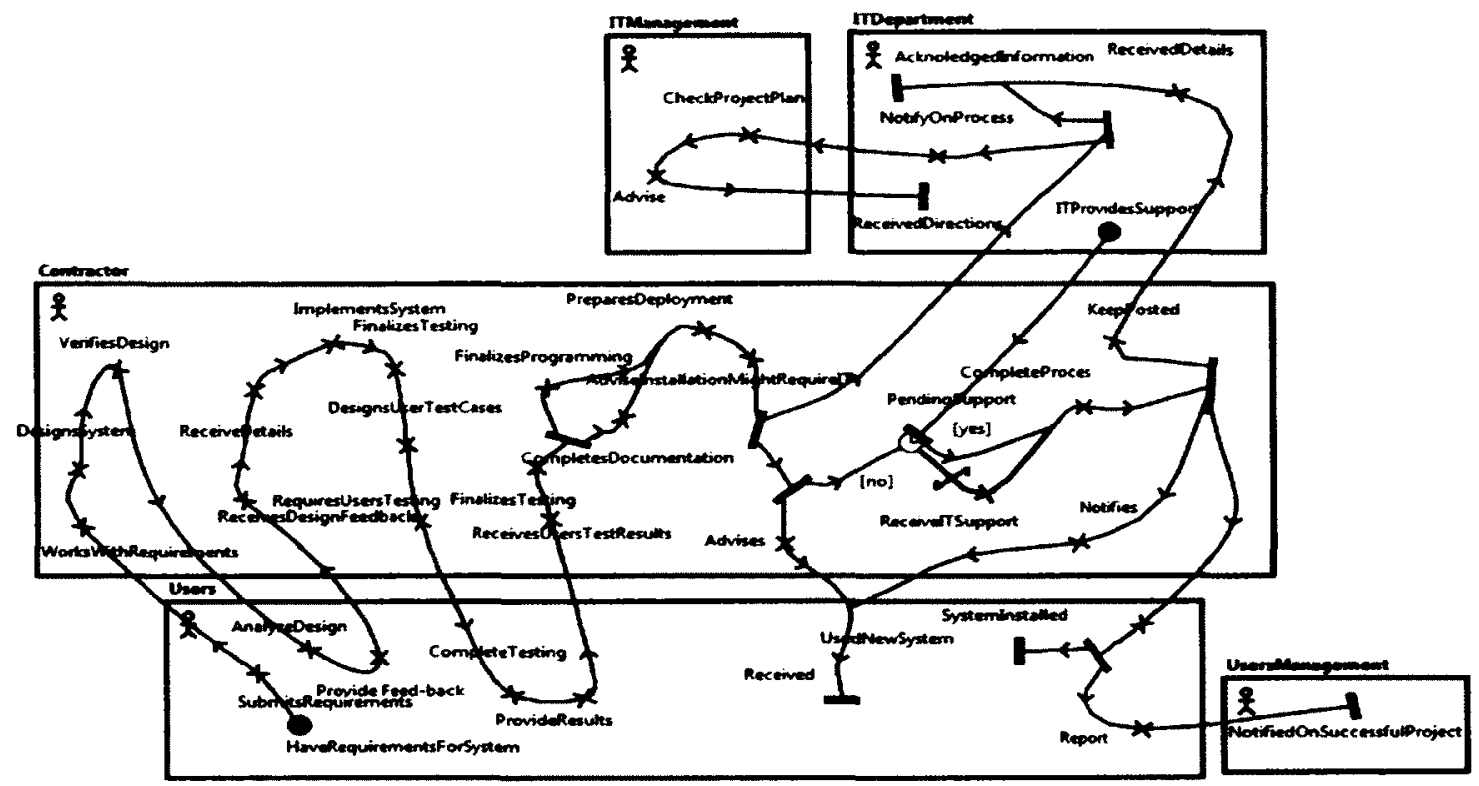

Figure 3.33: Overview Contractor work

3 UCM Scenarios

B (1)WorkingSystem_Request (6)

$E$ (2)WortingSystem_OtherDepartments BusinessAil

i (3)WorkingSystem_ContractorDevelops (14482)

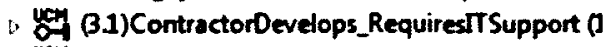

D 6 (3.2)ContractorDevelops_NoITSupport (14487.

Figure 3.34: Scenarios list Contractor
Work arrangements with the Users SO, ITD and $\mathrm{BD}$ (UM, ITDM, BDM) are to be arranged prior working out the Contract. Therefore if the Contractor will need assistance, the Users

will contact ITD to support the Contractor's work. This is required giving that the ITD is the main provider of software and technological services in the organization, and to ensure that work is done according to the system and Users requirements, and in respect of the IT regulations and standards. As indicated in the map Figure 3.33 waiting time is being taken in consideration, in case the Contractor needs to wait for an IT resource to be released from a previous task and become available for work.

\section{c.3. System maintenance/development view of Users work}

In this case, the development of new system features/maintenance work is suported by the Users, shall require minimum cost, and resolve time delays, staff overbooking and 
interference of goals. In Figure 3.35 we present the overview of the scenario and in Figure 3.36 we present the three scenarios that support this model. In Scenario (4.1) the paths are well executed (Figure 3.36 and Figure 3.37), with work part of operational

$B$ (4)WorkingSystem_UserDevelopsSystem

oy (41)Use-Develops_RequestApproved ApprovedTraining

planning and budget available.

(4.2)UserDevelops_RequestNotApproved

of (4.3)UserOevelops_RequestNotinProject, ReceivefaternalBudget

Figure 3.35: Summary scenarios, system maintenance

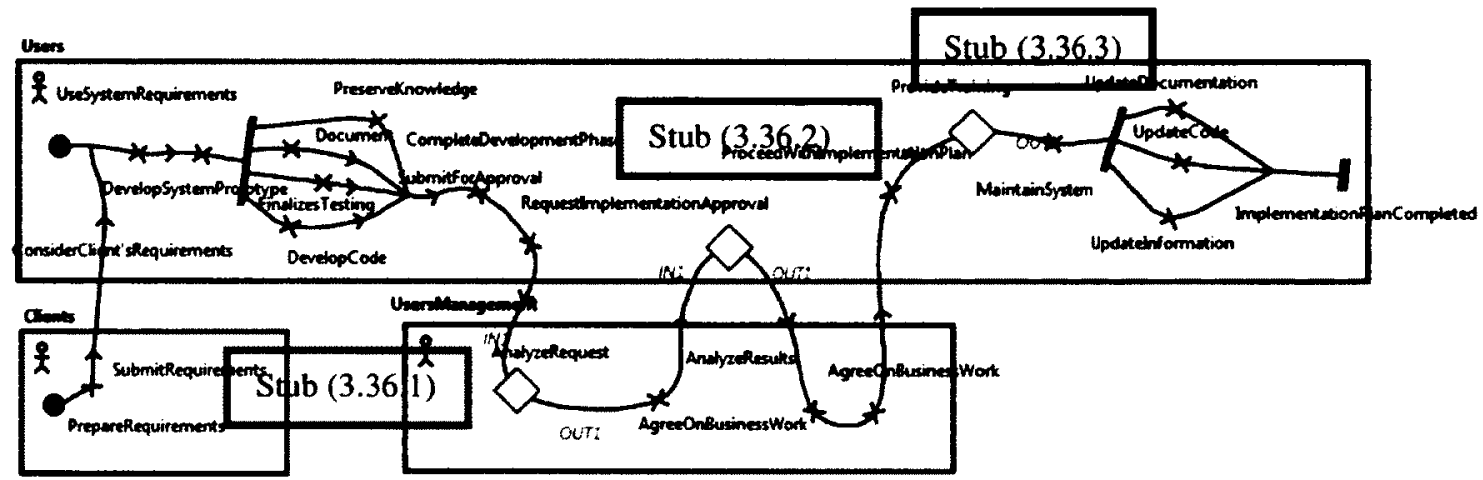

Figure 3.36: Overview of users maintenance work

In Scenario (4.2) the request could be ad-hoc/urgent giving that is not part of the operational plan (see Figure 3.37). To proceed UM might consult the Stakeholders to determine if there is budget available across the organization level, to cover additional training required by this work, as in Figure 3.38.

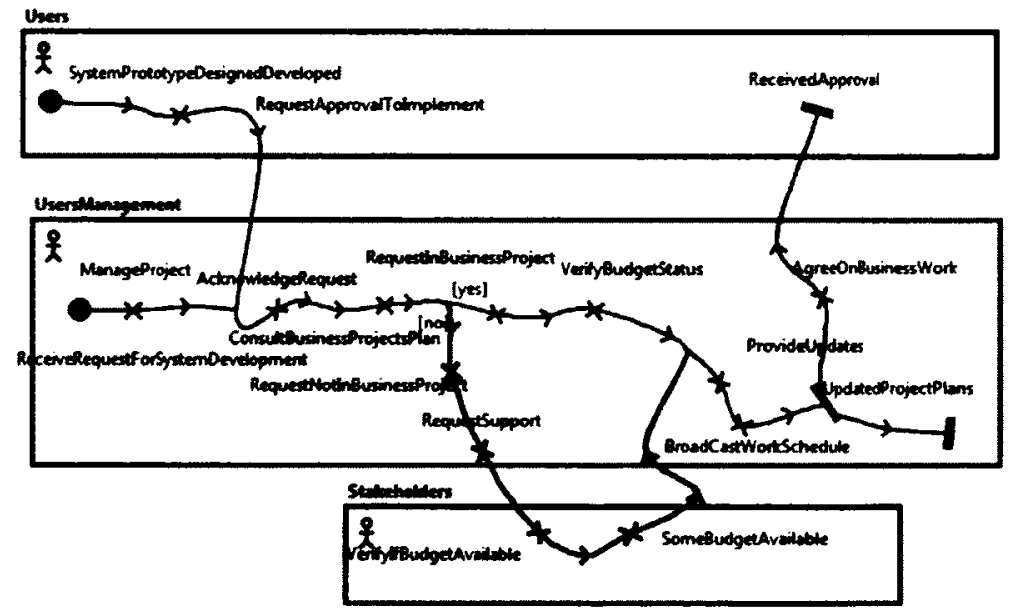

Figure 3.37: Users maintenance work, sub-map Stub (3.36.1) 


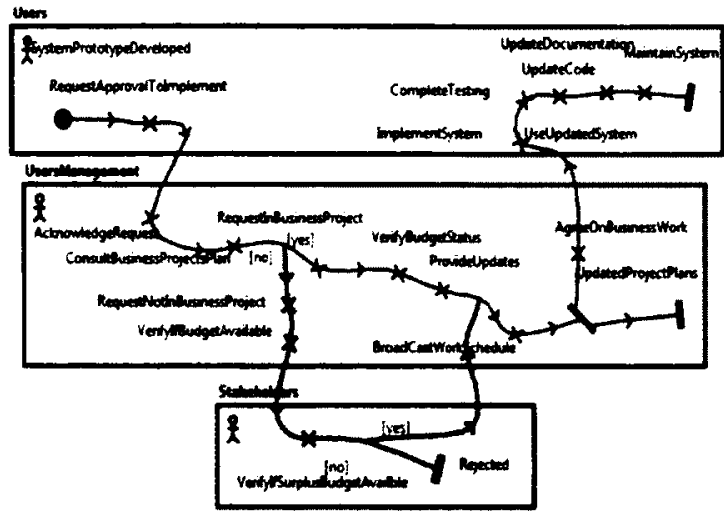

Figure 3.38: User work, request (Stub 3.36.2) implementation approval

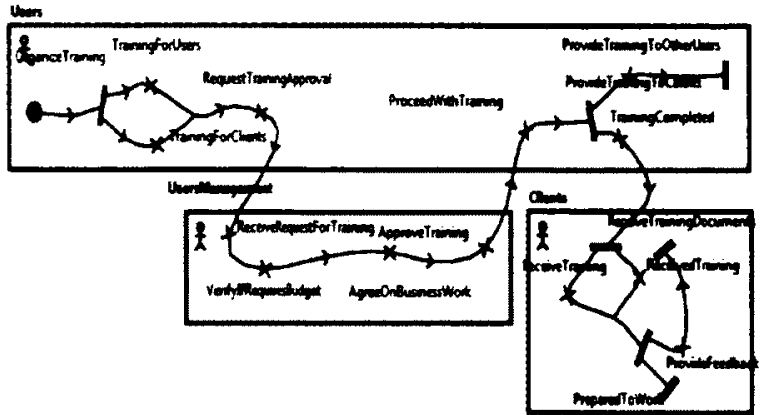

Figure 3.39: Users work, (Stub 3.36.3) training provided

\section{c.4. The Users sub-organization purchases an off shelf product}

For this case we are looking at two scenarios, presented in Figure 3.40: Scenario (5.1)

presents the case when the Users purchase the product respecting the technology and networking rules imposed by the ITD, while Scenario (5.2) presents the case when support from ITD is needed to install the product.

3 (a)WorkingSystem_Request (6)

3 (2)WorkingSystem_OtherDepartments BusinessAndr

3 (3)WorkingSystem_ContractorDevelops (1482)

3 (4)WorkingSystem_UserDevelopsSystem (6512)

3 (5)WorkingSystem_PurchaseProduct (13518)

1) yey (5.1) PurchaseProduct_Noll Support (13519)

o of (5.2)PurchaseProduct_RequiresiTSupport (13520)

Figure 3.40: Overview of purchase product

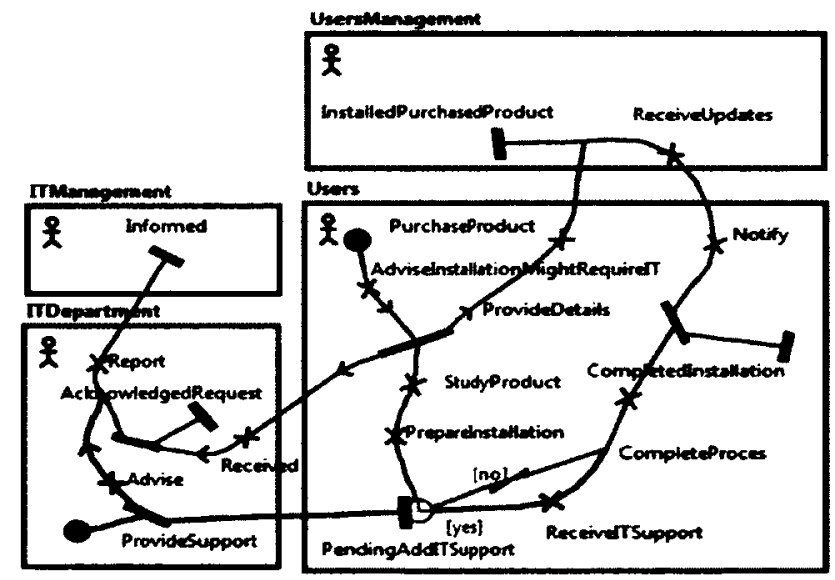

Figure 3.41: Use purchased product map

\section{d) System monitoring}

Monitoring is another key activity in the management of the system, and in our case it falls under the responsibilities of the ITSG, given that ITD manages and administers the Users system at the organization level. The Users have limited knowledge on how the 
system is being monitored, what type of monitoring processes are running and what are the monitoring results. The Users are not part of an automated notification system, therefore the only way they can receive information on the system status, and on any other system matters, by emails submitted by ITSG. It often happened that the Users learned about a system problem after it affected their work; for example they couldn't access specific functionalities and web pages; some reports were printed in a different template; the connection became slow and they had difficulties in running queries; one of the main Users servers went down and it took five days to have it restore. It is to the Users understanding that various monitoring processes are being set up by ITSG to audit the "data acquisition" [Sladic11] and traffic of records, to supervise "network elements functionality" [Sladic11]; to prevent, predict and identify "failure" [Sladic11], including "bottlenecks resulting from peak workload, staffing, and other resource limitations" [Zheng06]; to detect any potential malfunctioning applications and devices "based on data gathered during network monitoring" [Sladic11]; to identify "fault tolerance", ensuring that "collapses and local problems will not affect the overall" functionality of the "system" [Quan 11]; to manage the automated notification system, configured to monitor not only the system, but also the "system environment about changes in network functioning" [Sladic11]; to support "problem solving", monitoring of correction work, including the repairing of "broken devices" [Sladic11].

GRL graphs and strategies - We present this case with two GRL Graphs, described in Figure 3.42 and Figure 3.43. We see two possibilities of monitoring: first, is done by ITD, and in the second case, the monitoring is supported by the Users. In Figure 3.42 we present the Actors that could be impacted by monitoring; they are UM and Users, while in Figure 3.43 we are presenting important activities, tasks that are related to Users 


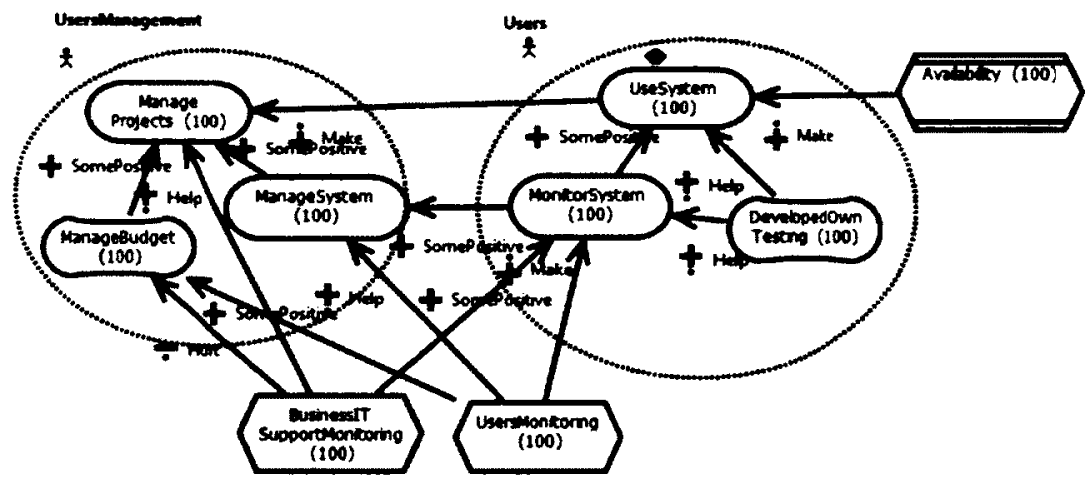

Figure 3.42: Users view of system monitoring

system monitoring: Automated Processing of Records, Identification of Bugs, Additions;

they will contribute to the realization of the Users goals also impacted by monitoring:

Users Use Production, Identify system Problems. Monitor Production, Monitor

Corrections, Monitor System.

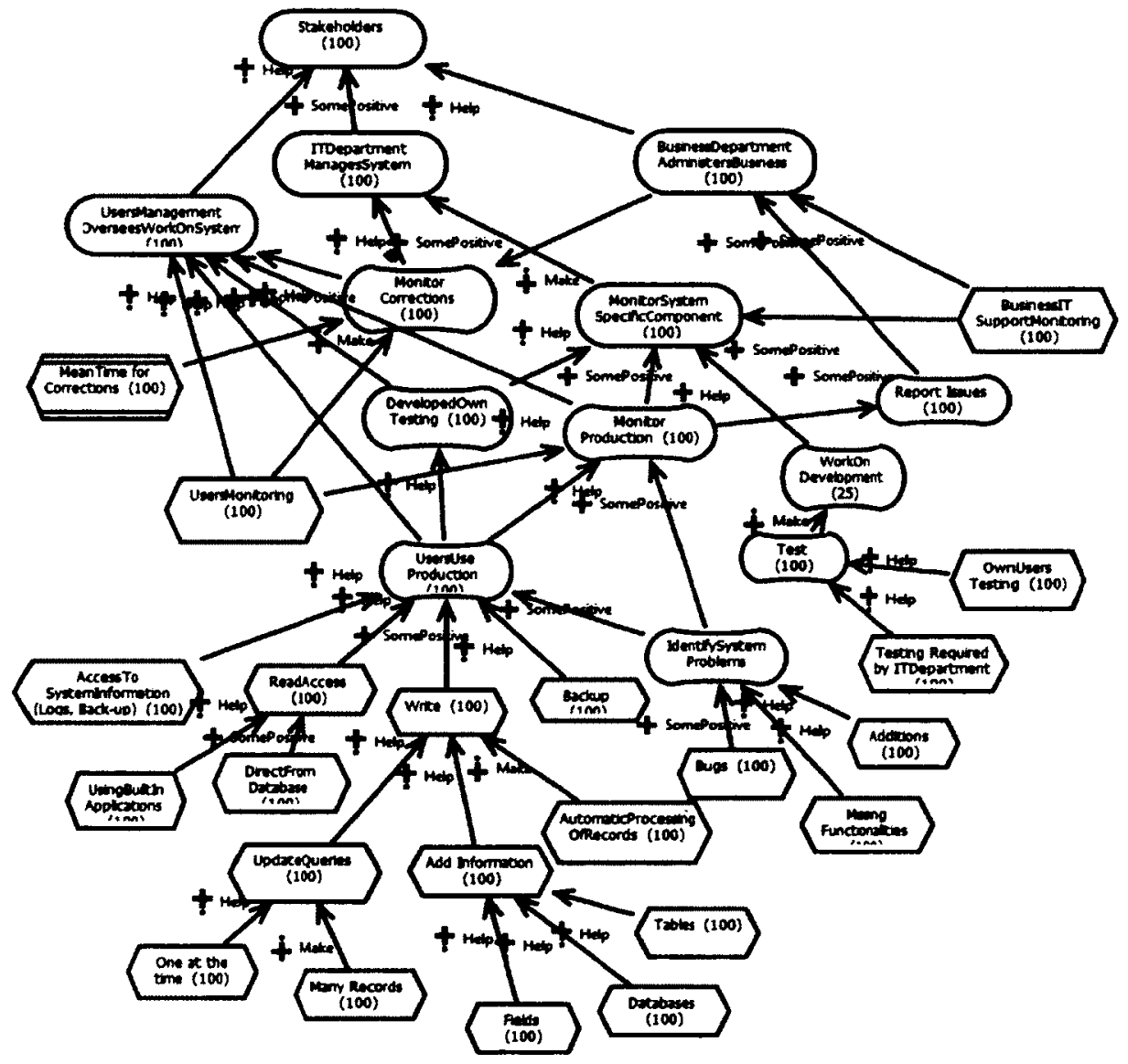

Figure 3.43: Detailed graph of Users view on monitoring 
UCM views and scenarios - There are different ways to monitor the system and this is reflected by the number of scenarios we have created and summarized in Figure 3.44.

First scenario executed below (Figure 3.45 and Figure 3.46) is showing a high level

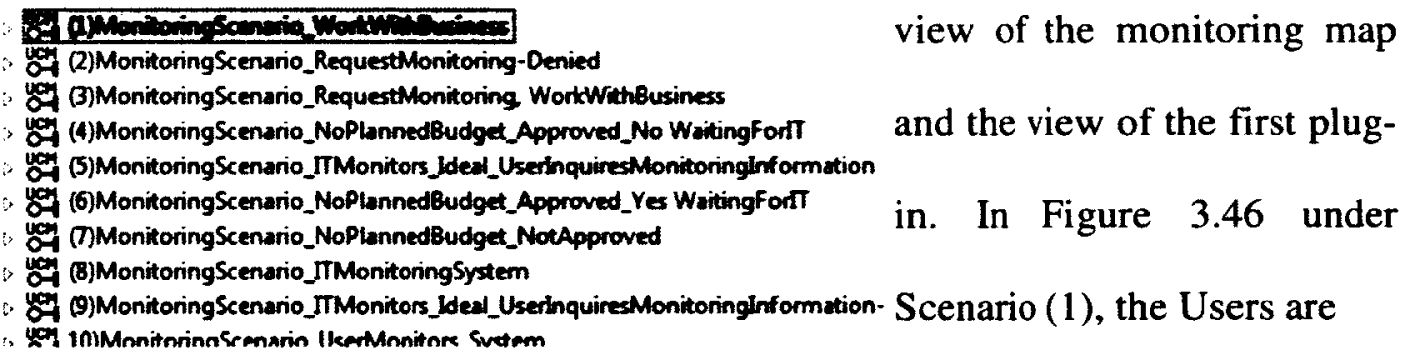

Figure 3.44: Scenarios list, system monitoring

preparing their needs in monitoring; after they are approved by UM, the Users request

will be submitted for action to $\mathrm{BD}$.

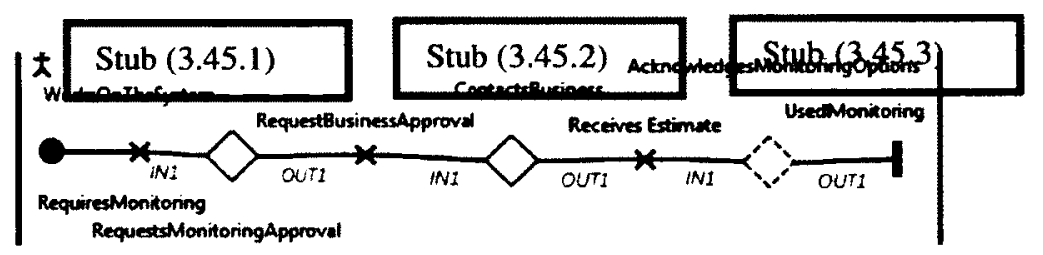

Figure 3.45: High-level map, system monitoring

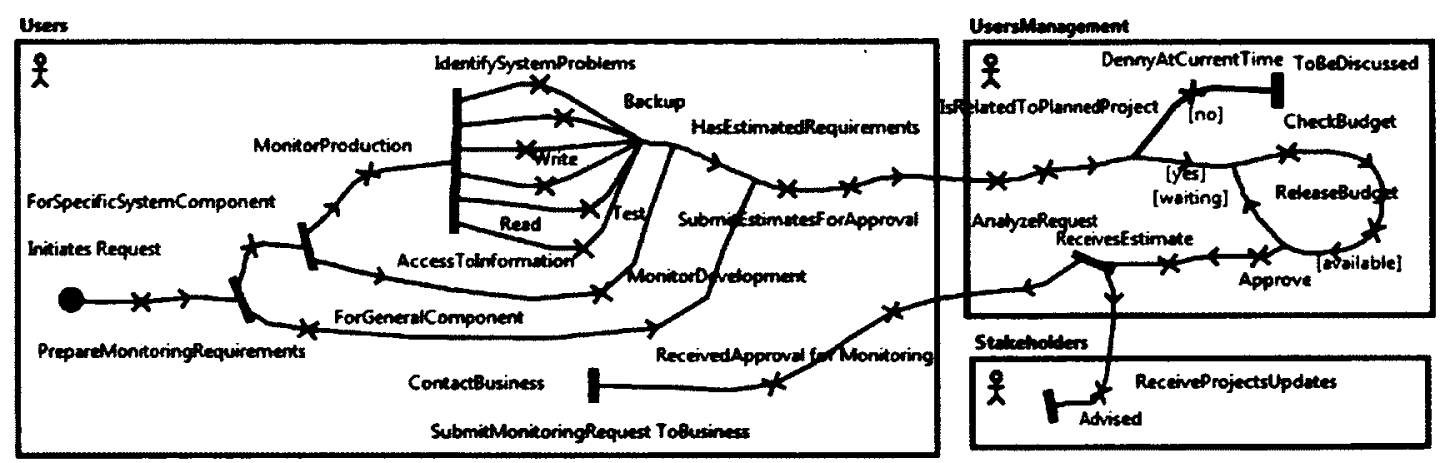

Figure 3.46: View of RequestBusinessApproval (Stub 3.45.1)

Scenario (2) is presenting the alternative path when the request has not been previously captured in the operational plan; therefore the approval has been denied by UM and further discussions will be conducted on this matter. In this case the request has not been 
submitted to BD for any follow up. Scenario (3) continues the process started, and presents the map hosted by the second stub (as presented in Figure 3.47). The request is part of the operational plan, has budget assigned, therefore BD will complete the request with their information and will further address it with ITD for action. Scenario (5) for example takes us further, allowing to check out one of the maps bound in the third stub (see Figure 3.48, Figure 3.49). The ITD received Users request regarding monitoring and will check with system management if these practices are being accepted.

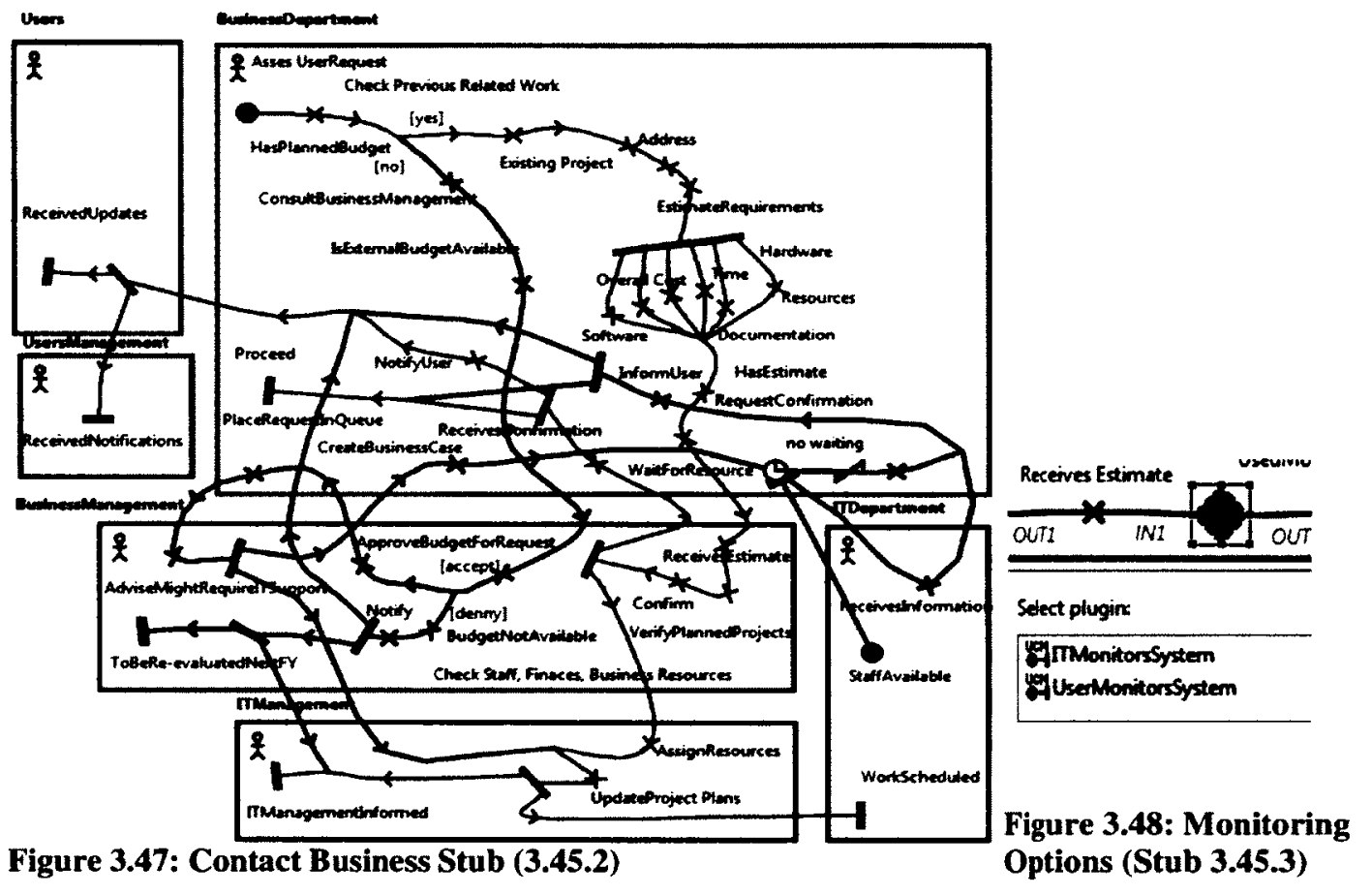

If yes, they will monitor the components/activities required by the Users and return the results to them. Else further discussions might be required. 


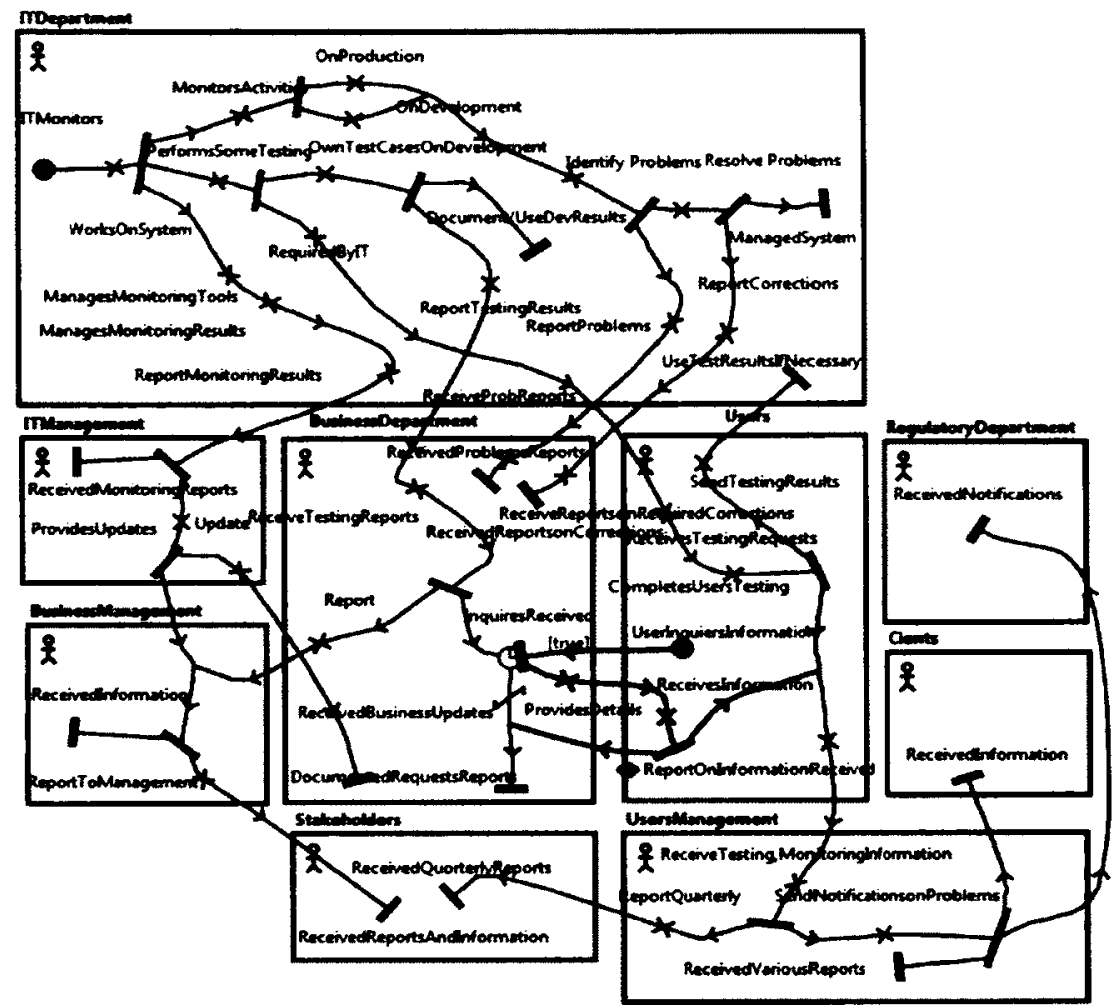

Figure 3.49: IT Monitors Users system (Stub 3.45.3) 


\section{Proposed System Model}

During the modeling of the working system presented in the previous chapter, an evaluation of its performance was done (as discussed in Chapter 5) and we realized that the system has various inefficiencies. A new version, called the proposed system is described in this chapter and its URN model is presented. The next chapter will discuss comparatively the evaluation results of the working and the proposed models.

\subsection{What is wrong with the working system}

A few years ago, the Users system had to undergo a refurbishing process, due to outdated technology and software applications used in the system. The changes the system went through were based on decommissioning the outdated technology and replacing it with up to date equipment, including servers, computers, network devices and technology. All software platforms and applications have been also removed and replaced with most recent and performant products available on the market. In the meantime, a few legislations related to the maintenance, updating and refurbishments of systems that contain and administer data records have been changed, and therefore had to be included in the matters to be resolved with the refurbished system. Aside many changes, the records and the data from the database have not been modified during the refurbishment process. The Users are not the owners of the data, they are just administering it and using it to provide information to Client's requests. After the refurbishment process had been completed, and after testing and working in parallel with both the old and the new system, the old system has been decommissioned and the new system replaced it in all Users activities. The refurbished system is our case study. At that time, the Users had to 
handle not only the benefits of working with a new system, but also the challenges brought by the many changes of the Users system, including hardware equipment and software applications in their own sub-organization and at the organizational level, under the management and administration of the business and information technology departments. This was part of a centralization process conducted across the organization. Even though there were many other SO that had their systems transferred, there were few departments that were able to keep the systems under their own management and ownership. The transfer of the Users system had an impact on the way the Users worked. With the refurbished system, they have restricted access to the database they used to manage and administer for more than 35 years; the access is granted through a secure web application, with predesigned queries and applications. Aside from the system, they also had to transfer the operational business activities of their system to the business department. Therefore BD took the lead in managing business activities and any work with the current system. The Users understood that this was the new approach at organizational level, and they have complied will all new imposed requirements. Since then, there were often times when the Users needed some of the old queries and applications in order to complete some data analysis and reports requested by the clients. During testing, they have learned that, while retrieving data records from the database through predefined connections, some of the table data definitions were not preserved well, and the data extracted came with a different definition type in the application that allowed them to read the records queried from the database; this made them use the limited predefined queries, while waiting to install a new application for reading queried results. Then they have learned that it was challenging to work without always being 
aware if maintenance activities were running on the system or on the network. Also, running complex queries on large data sets, required in some of their reports, could take up a lot of resources, causing server problems. The Users had to learn new requesting and reporting processes, in which most of the requests concerning the system had to be submitted first to the BD for investigation. If the investigation reports, which did depend on the operational plans of the Users $\mathrm{SO}$ and of the $\mathrm{BD}$, on budget status, and possibly on legal requirements indicated positive results, the requests would be forwarded to the ITD for processing. Otherwise, they could be bundled in projects to be addressed at a later date, or could be deferred to further discussions and analysis. Given that the two departments $\mathrm{BD}$ and ITD also provide real time services to all the other SO across a complex distributed system, except for the few SO that own their own systems, the Users found many times that their requests were placed in a waiting queue, regardless of their level of priority from the Users' point of view. This introduced delays in getting their requests processed, which also introduced delays in the response time for some clients. The Users learned that is difficult to wait between a few days and a few weeks to have a part in a report updated; or a few days to have one of the servers restarted after a failure. The Users learned that it is difficult to work with no access to system and applications monitoring, with no customer service outside the office hours in working days, in weekends and during statutory holydays.

Based on the disadvantages of the working system, this chapter proposes an alternate system, in which some tasks, goals and responsibilities handled by ITD and BD, have been transferred back to the Users. Next we will evaluate both models and compare the evaluation results. We believe that for the proposed system the evaluation results will 
indicate a better realization of the Users' goals and higher satisfaction levels than for the working system. By modeling, we are looking to provide a favorable workframe to describe the system and its operational business requirements. We intend to share the model and the evaluation results with the Users' management, given that the URN modeling language is an intuitive notation with good tool support, that allows to visually present the system with all its components, without having to go into technical and technological details. We have interest to learn how inter-departmental work activities and relationships impact the realization of departmental objectives, to learn how to tune up processes and shift activities when necessary, to ensure a uniform realization of the goals across the organization for each SO.

Since in this case study we are interested in how the model helps the understanding of the system rather than looking for validation with quantitative external data (which is difficult at this stage), we will use KPI inspired from the real system, using values based on real facts and work experience. We consider that a system model is valid if it represents requirements, considers legal aspects and regulatory specifications imposed by $\mathrm{RD}$ and $\mathrm{LD}$, and integrates with the stakeholder's objectives established at the enterprise level. We study the evaluation results to determine if the model fits its purpose, to identify deficiencies, to understand their causes and impact, and to update the model to improve the solutions and to close the development loop.

\subsection{Modeling and analysis}

As introduced in Chapter 2, goal modeling and evaluation could help determine how goals can be realized, at what satisfaction level, and if there are any potential conflicting 
situations or unsatisfied objectives that could appear in any given context [Amyot03a]. Our proposal is to model and evaluate the system at an early design phase, after defining the requirements (see Fig.4.1) and before proceeding with the design, instead of postponing the evaluation for later, in the testing phase. Late detection and fixing of problems could require costly changes to the requirements, design, use cases, implementations and test plans. The diagram in Fig. 4.1 also helps to indicate the activities managed by BD and ITD compared to the activities managed by the Users.

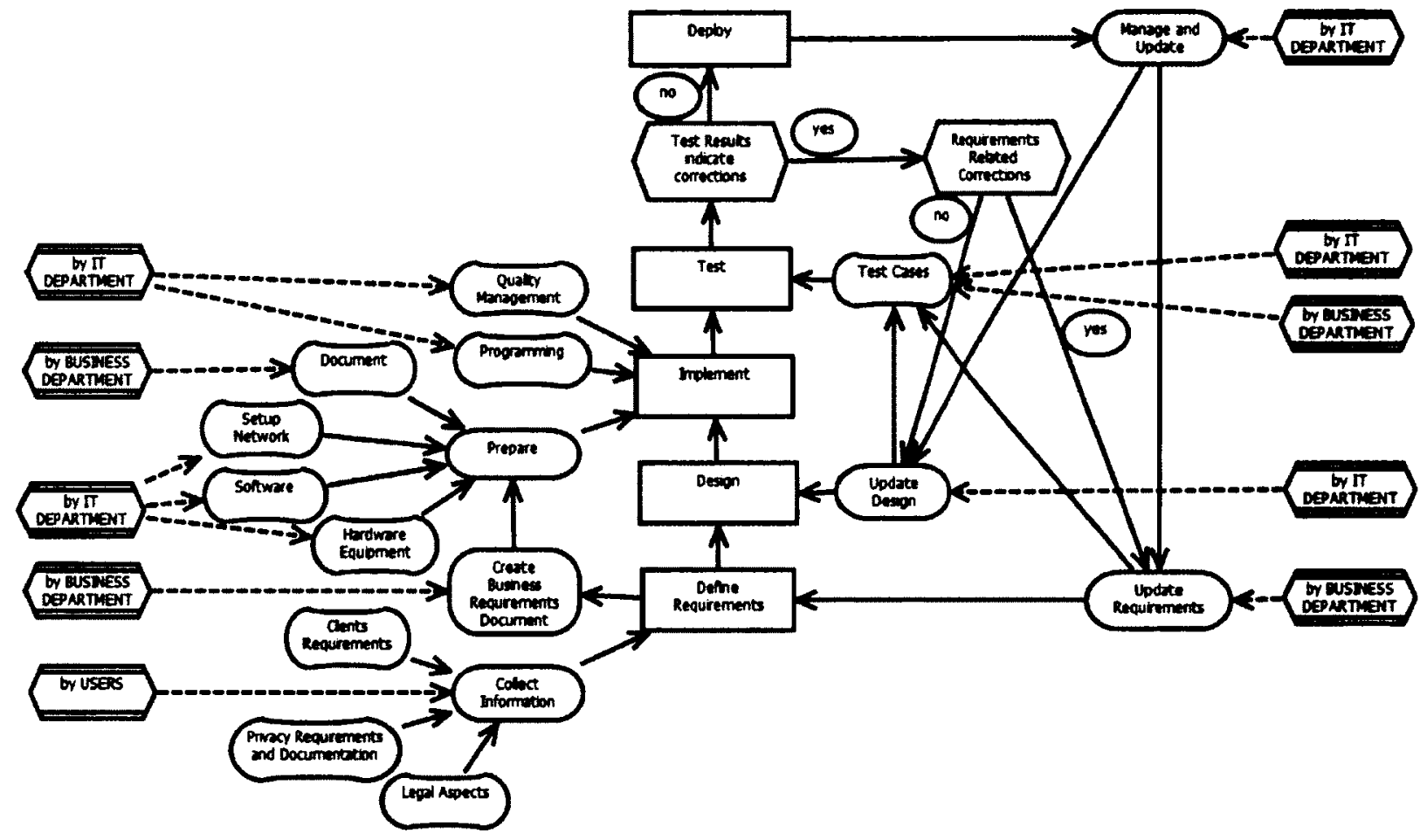

Figure 4.1: High -level diagram presenting in details BD and ITD services

These responsibilities are labeled by IT Department and Business Department. This transfer will help evaluate the model, estimate the goals realization as seen in Figure 4.2. Next step is to evaluate the new model for the proposed system, analyze the results to verify if there is any improvement in the realization of the Users goals and satisfaction level. The next diagram in Figure 4.2 also helps to explain what type of reporting and 
processing loops are running in the Users system in order to complete a task or obtain response to a request.

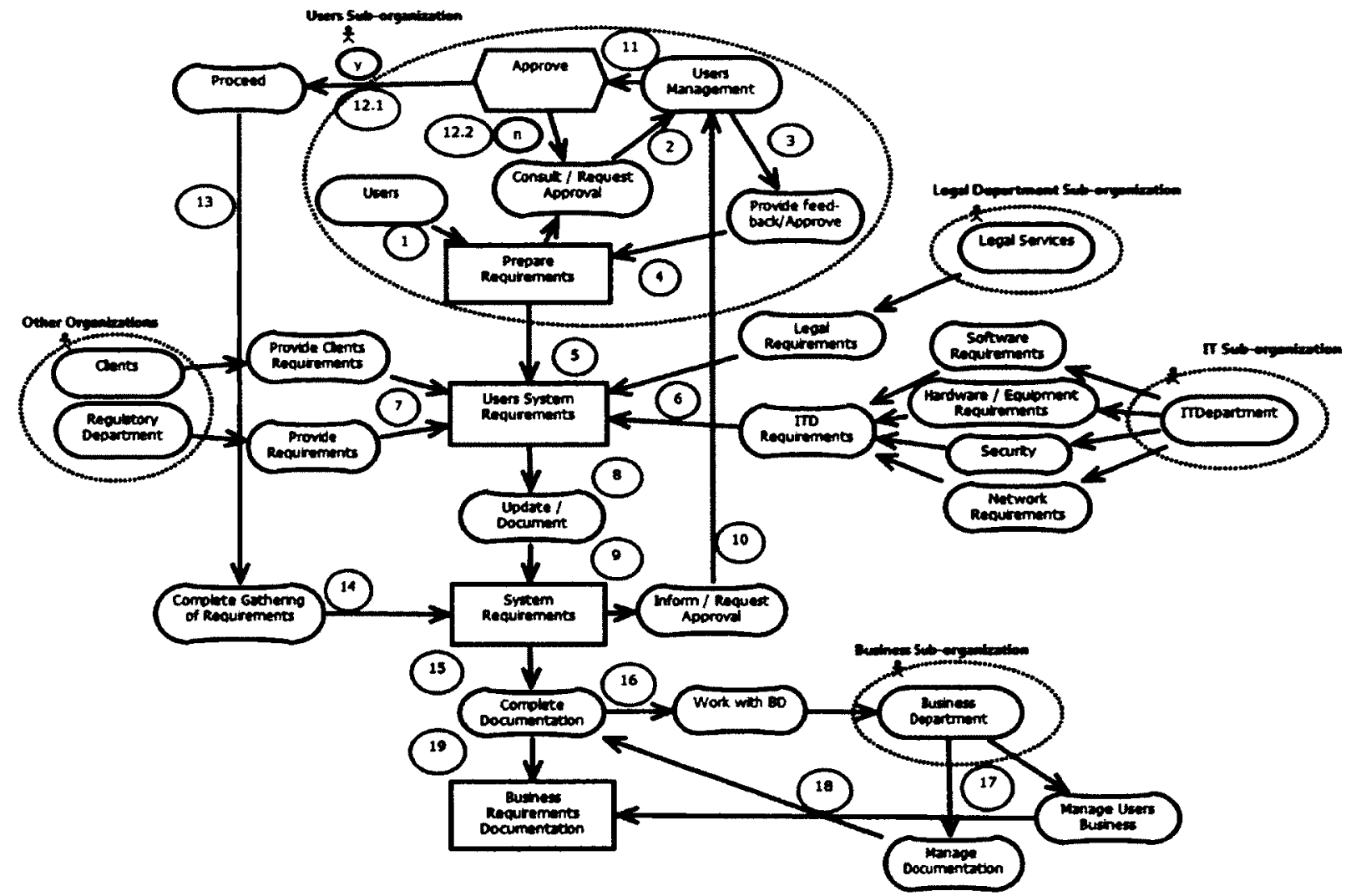

Figure 4.2: Example of information propagation in the system

According to the work processes, it takes nine steps to gather system requirements and eleven steps to have them approved; fourteen steps to have them completed, eighteen steps to have the operational business documents updated, with information exchanged, analyzed and approved across different SOs. This confirms that the inter-departmental interactions are often the source of overheads we are looking to reduce in the proposed system. We will demonstrate with the evaluation results how we have succeeded.

Given that the system is being administered by two other SOs, BD and ITD, we have understood that there are knowledge limitations for different actors, not only limitations 
in what they can do. Therefore, we will model the functionalities of interest for the Users, evaluate the model and present the UM with the results. This will give the Users an opportunity to analyze with its manager and other stakeholders if their goals are realized at an acceptable satisfaction level.

\section{a. Users activities and work on the system}

The corresponding model of the proposed system for this case has been obtained from the complex model of the working system presented in Chapter 3. Given that the most important activities and responsibilities previously managed and completed by BD and ITD are transferred now to the Users SO, we expect to see a simplified model, with fewer Actors, fewer activities that are crossing the borders from Users to BD and ITD, all of this leading to better goals' realization.

For example, in the original GRL graph corresponding to the working system, Manage Code was a main goal for Actor IT Department (also known as ITD), and Maintain Documentation was a main softgoal for Actor Business Department (also known as BD). Both elements have been extracted from the original GRL graph, and brought as tasks to the Users Actor, in the graph for the proposed system presented in Fig.4.3. The Users handle the Identification/Resolving of issues, which now includes in the proposed system former goals like Monitor Projects and Resolve System Issues. If in the working system graph, goals like Assist in Identifying/Resolving Issues would contribute to the realization of Manage Projects (from BD) and Resolve System Problems (from ITD) now it contributes to the realization of Manage Objectives and Manage Group Projects from UM. Giving that there will be less reporting and addressing of requests with BD and ITD, as Users are in charge with maintaining the system, and operational business activities, 
we have excluded some Actors like BD, ITD, BDM and ITM from the new GRL graph, and kept LD (with RD) Clients and Users' management.

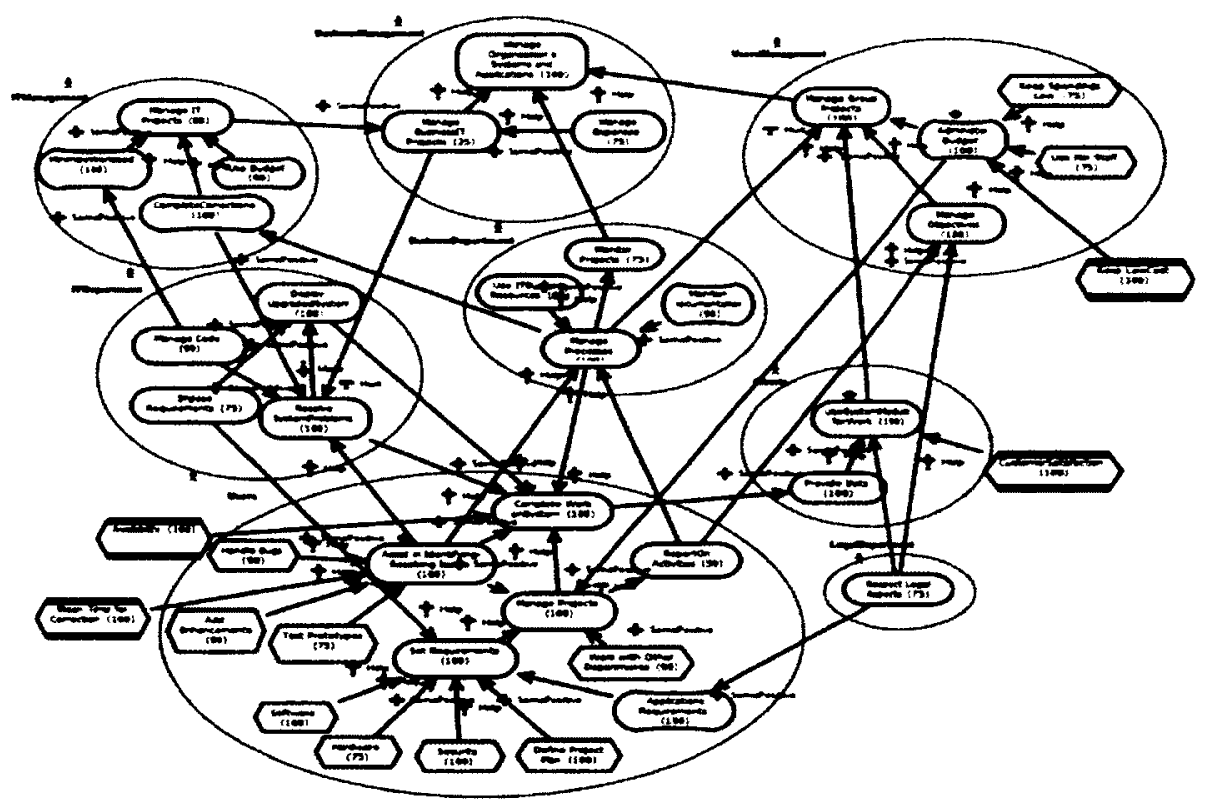

a. GRL graph for the working system

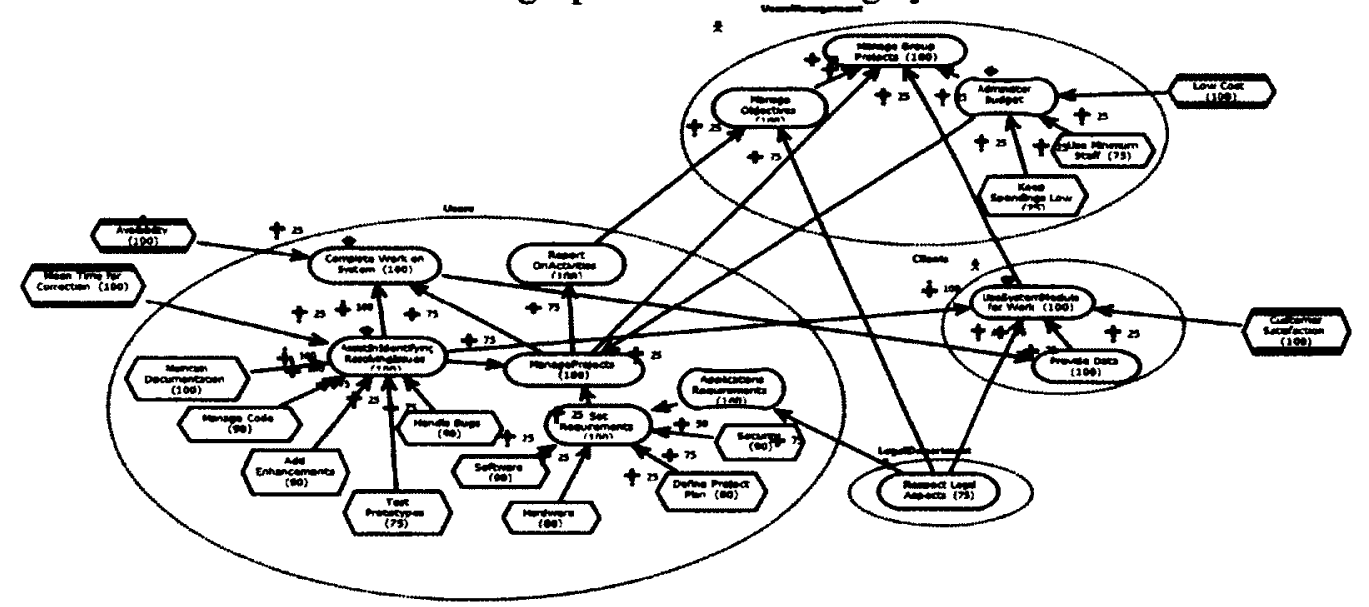

b. GRL graph for the proposed system

Figure 4.3: Comparing the GRL graphs for the two systems

We also see a lower number of strategies and scenarios and simpler UCM models, giving that the Users are now managing their own system. In Figure 4.4 we compare the UCM hosted by Reporting Issues stub corresponding to both systems. We can see fewer Actors in the proposed system diagram, simpler maps and a simpler flow of responsibilities. 


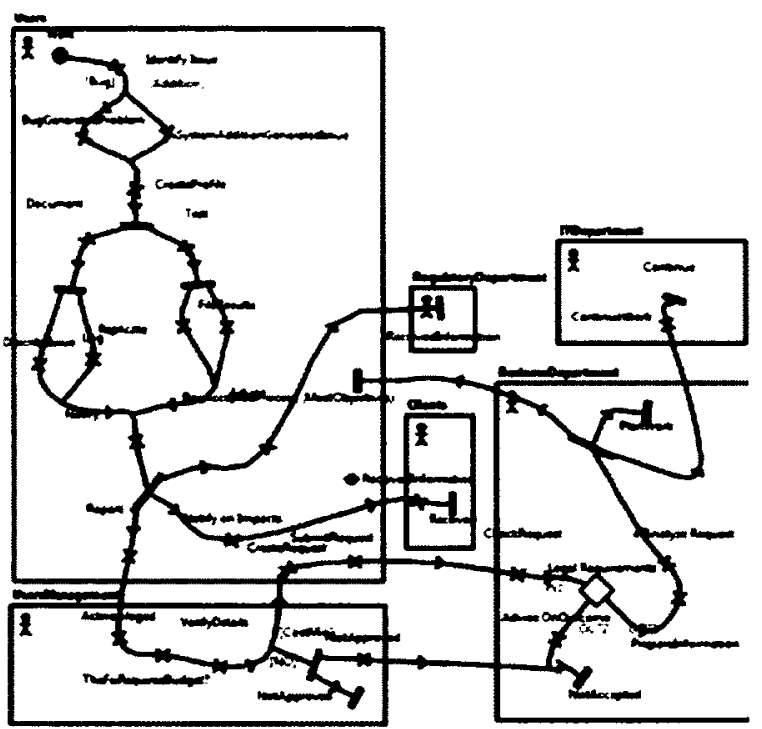

Reporting issues, working system

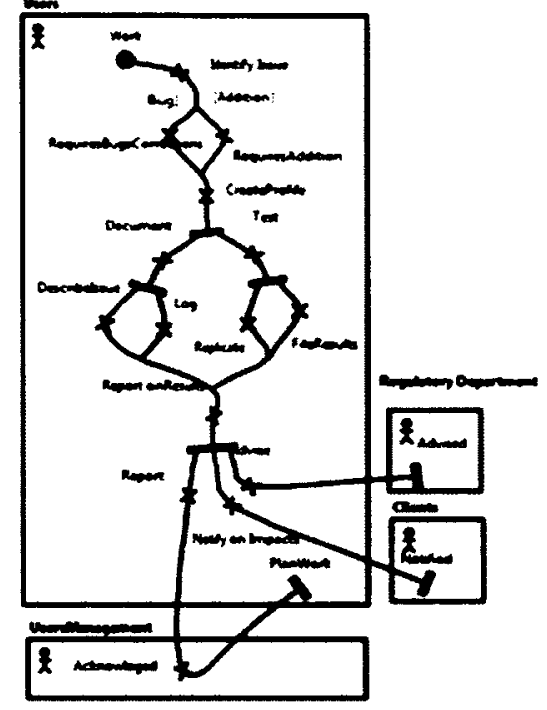

Reporting issues, proposed system

Figure 4.4: Reporting issues: comparing the two models

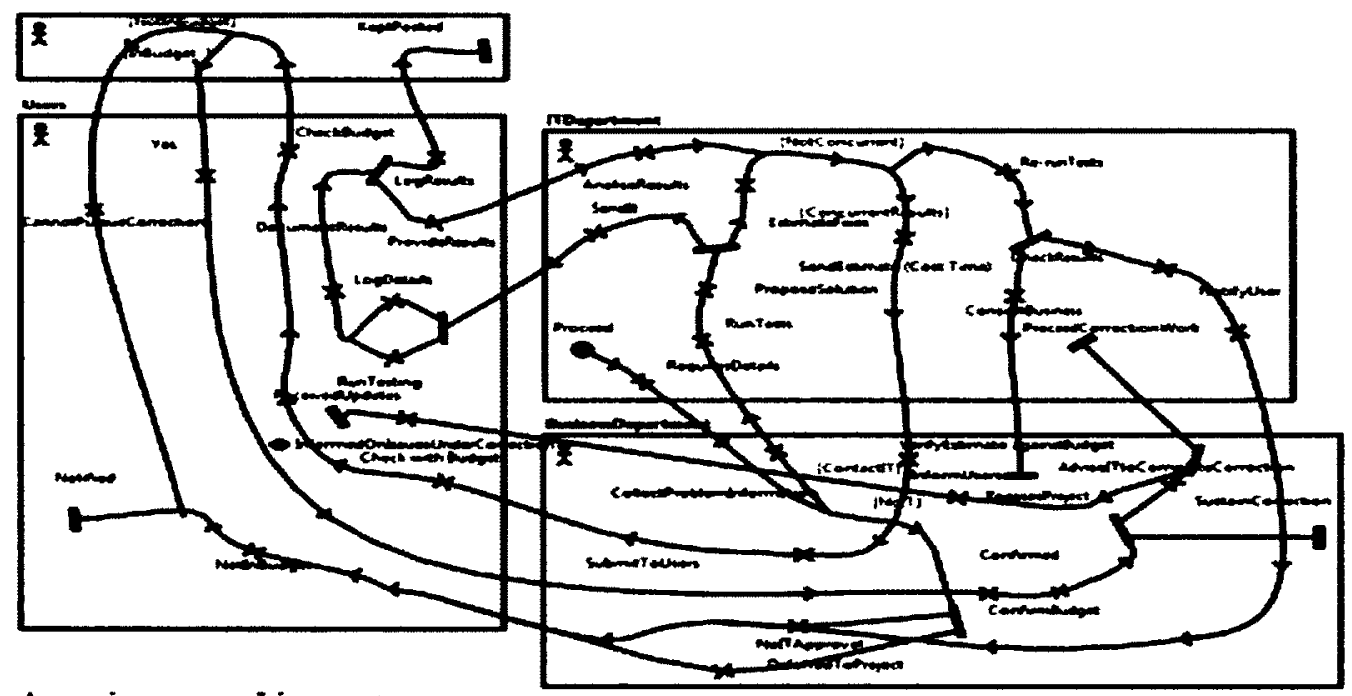

Asses issues, working system

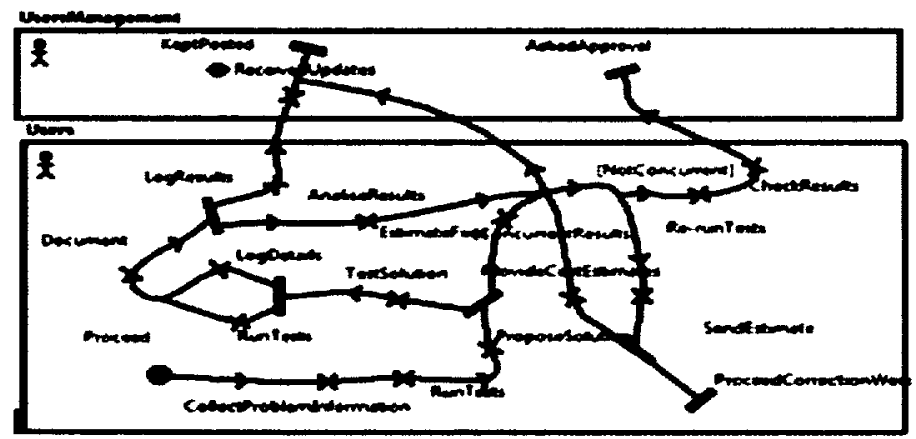

Asses issues, proposed system

Figure 4.5: Asses issues: comparing the two systems 
It is in the Users interest to realize the goals at the best satisfaction levels possible, in the Satisfied to Weekly Satisfied levels. We should find also the best strategies for evaluation, as described in Chapter 5.Figure 4.5 is comparing the plug-ins hosted by stub Asses Issues in the working and proposed systems. We can notice shorter paths in the latter, fewer interactions crossing the actors' borders, fewer actors and fewer responsibilities, less complex and easier to follow maps for the proposed system. All of this is a consequence of reducing the level of responsibility sharing and interactions between SOs.

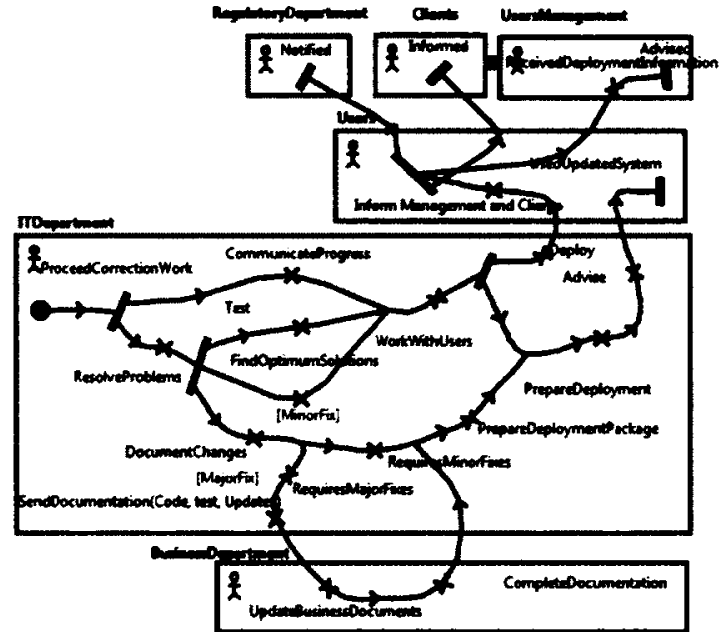

a. Prepare deployment: the working system

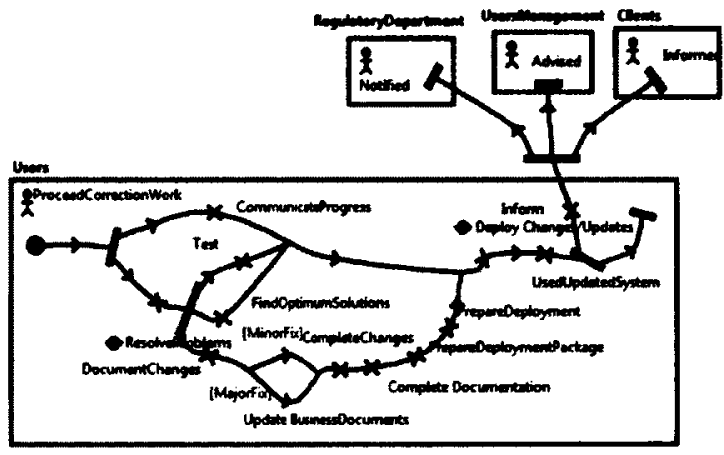

b. Prepare deployment: the proposed system

Figure 4.6: Compare deployment process on the two systems

The deployment of changes (see Figure 4.6) is also a simpler and clearer process in the proposed system. If in the working system, the Users barely had any responsibilities, in the proposed system, the deployment activities previously shared and managed by ITD and $\mathrm{BD}$ are now concentrated in the Users $\mathrm{SO}$, and are to be completed by the Users.

\section{b. Administrative information propagation through the system}

The GRL graph of the proposed system has been obtained from the GRL graph of the working system, by extracting all the tasks and goals related to Users activities, and by 
re-shifting the contribution of the goals, once the BD and ITD had less participation in running the Users system. Therefore we can notice in Figure 4.7 that the goal Resolve Requests would contribute to BusinessManagement ManagesProcesses goal in the working system, while in the proposed system it contributes to UsersManagement ManagesProjects goal. In the working system graph, the Reporting of Issues would be done through the $\mathrm{BD}$, which would manage the Correction Schedule and Manage Corrective Actions that would connect to ITSCmanagesSC, contributing to BD's goals. On the other hand, the Users have monitoring capabilities in the proposed system, which contribute to the realization of Correction Schedule and Manage Corrective Actions goals, which now contribute to Users goals, such as Resolve Requests and UsersReport on CompletedWork.

We can say that all the efforts invested by the Users in reaching goals and resolving objectives are concentrated and are beneficial to the Users and their own SO.

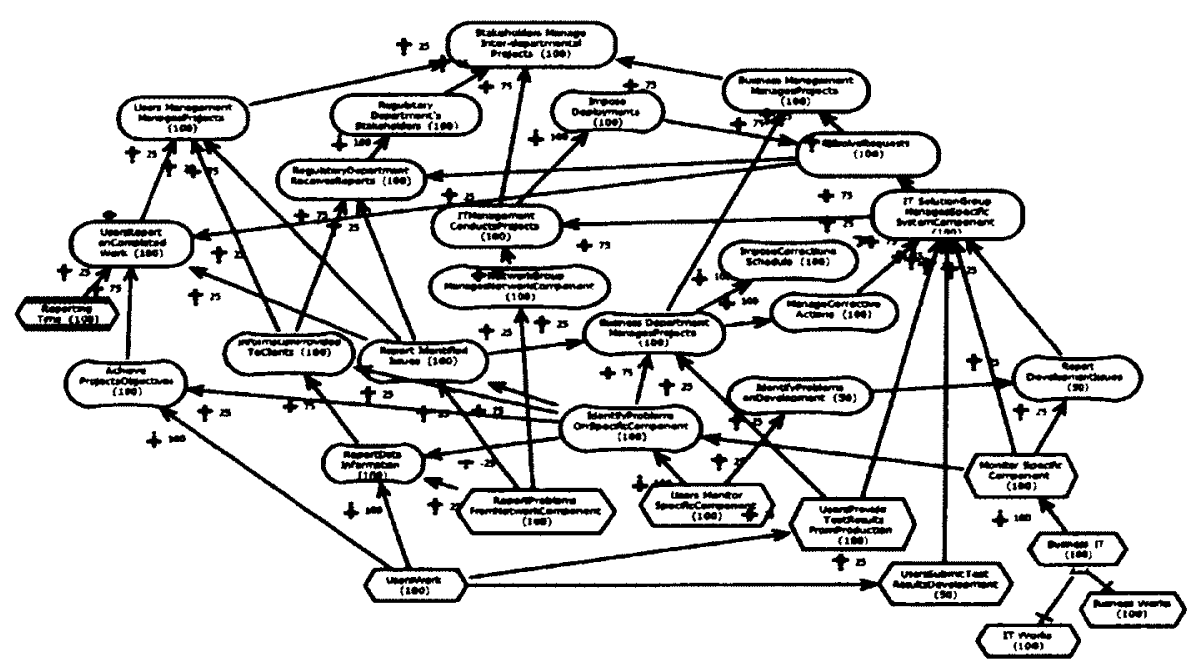

a. Administrative information propagation in the working system 


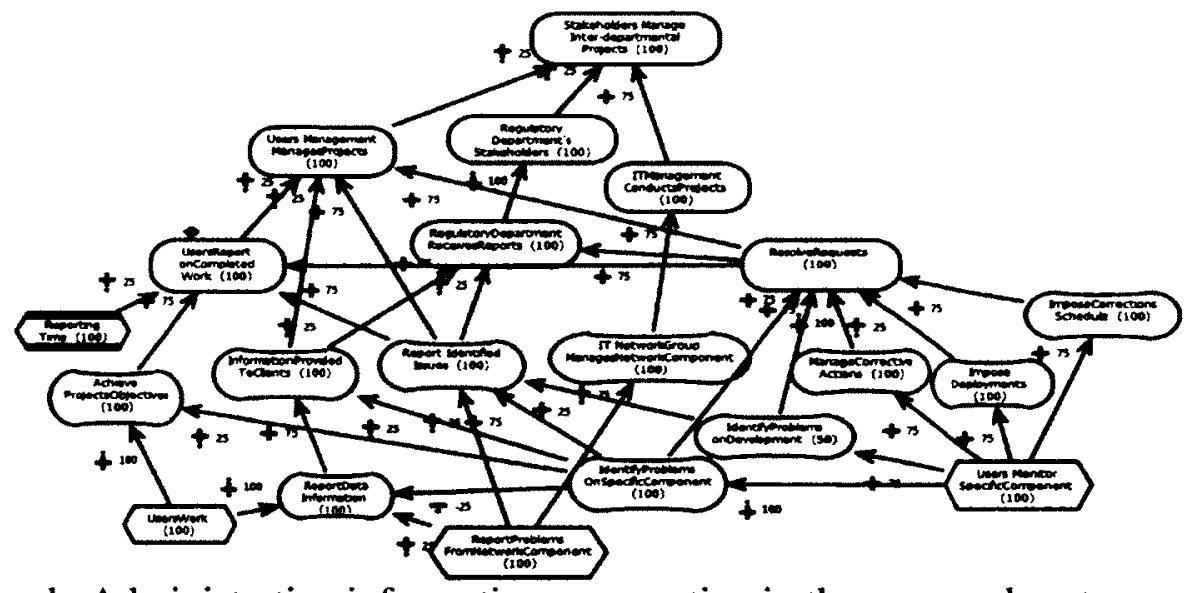

b. Administrative information propagation in the proposed system

Figure 4.7: Compare administrative information propagation on detailed GRLs

Next we analyze the plug-in maps corresponding to stub Work on System for both cases.

By comparing the maps in Figure 4.8, we can note that there are fewer Actors and stubs in the new system, due to fewer interactions with the other two departments. The Users are now in charge with managing CARs and related responsibilities (they own Manage CARs stub). In Figure 4.9 we can see that in the working system, the Users role was mostly to provide feedback to IT and notifications to RD and Clients.

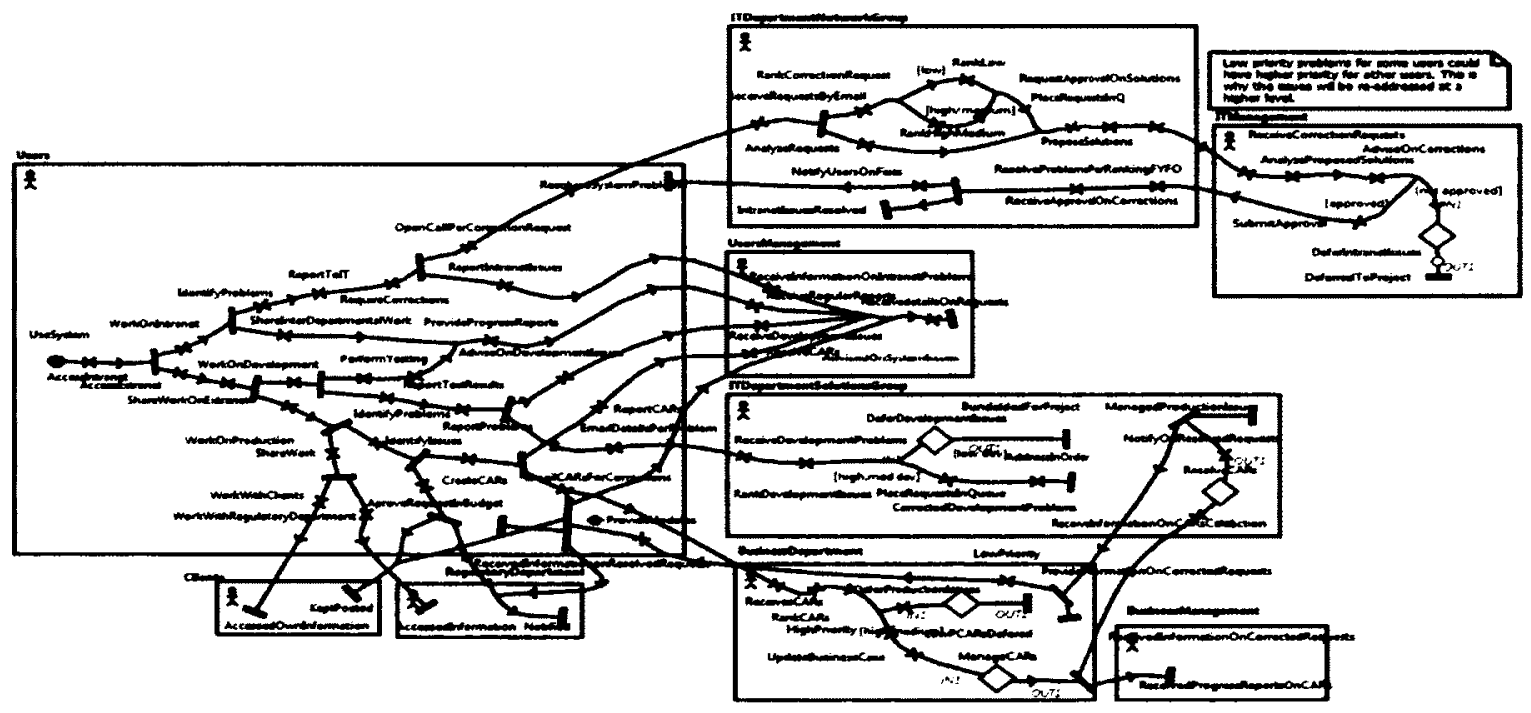

a. Information Propagation in the working system 


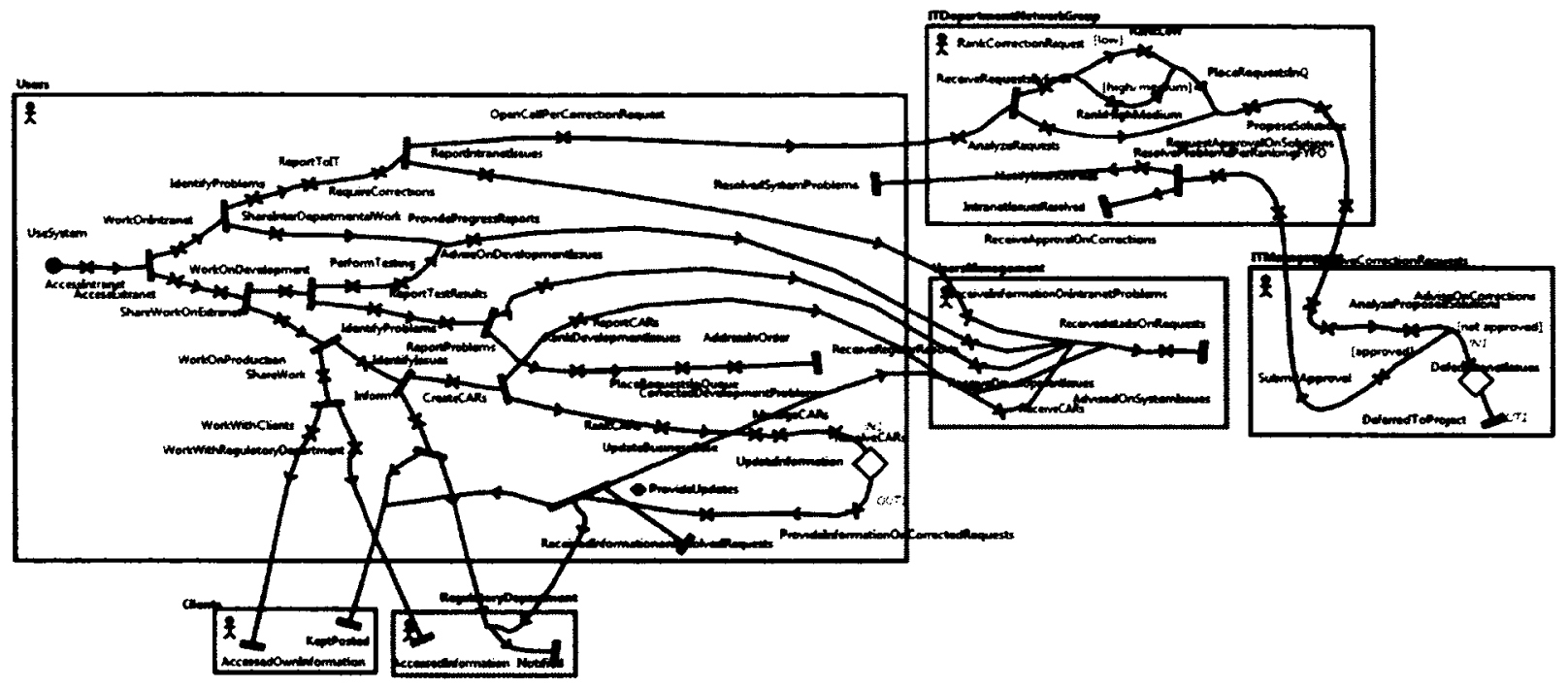

b. Information Propagation in the proposed system

Figure 4.8: Compare administrative information propagation UCMs in both systems

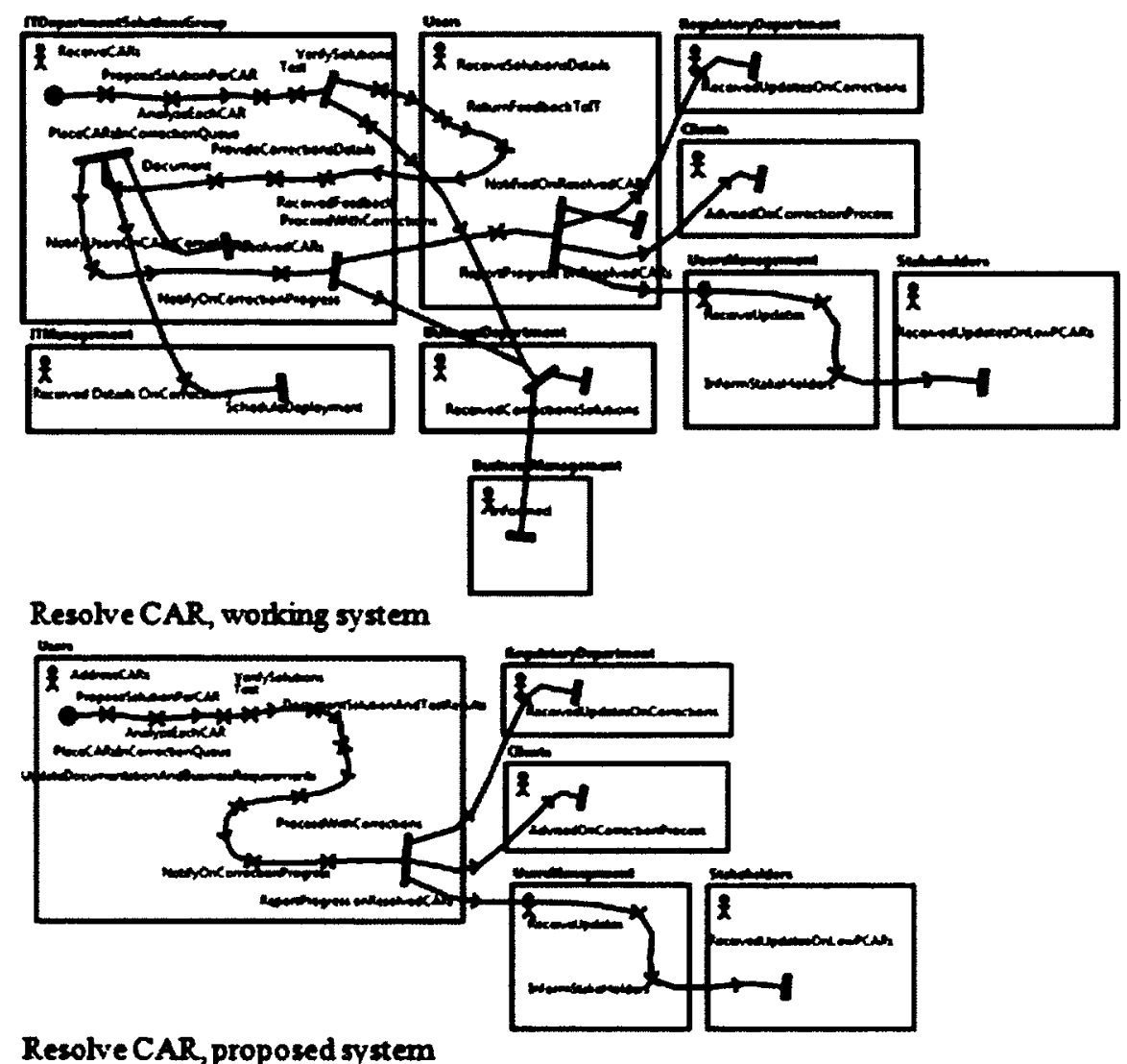

Figure 4.9: Compare handling of issues maps for both systems 


\section{c. First test then deploy}

In Figure 4.10 and Figure 4.11 we look at the high-level, and also at the detailed GRL graphs for both system versions. The graphs for the proposed system do not contain now the goals related to activities from the other departments, as the Users are in charge with
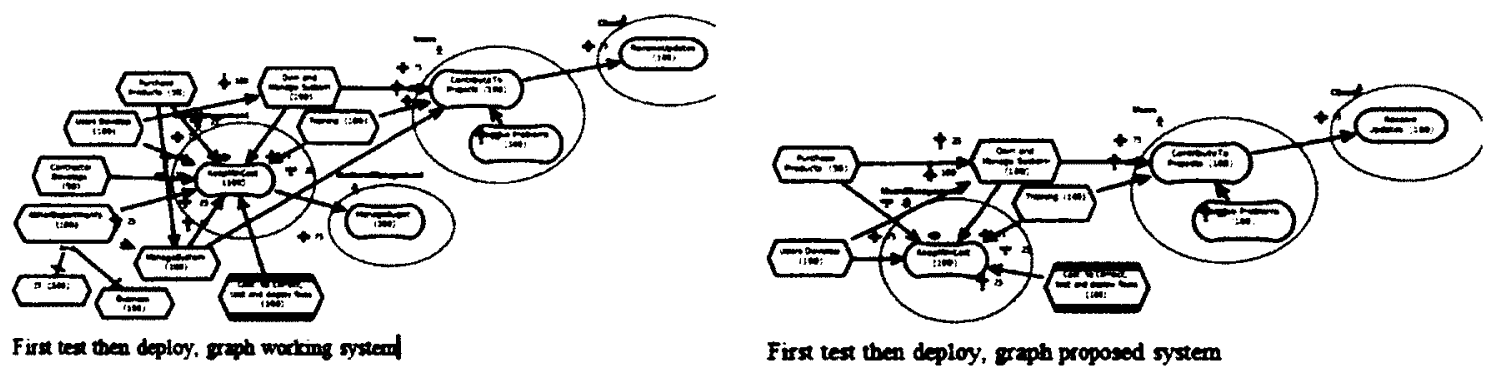

Figure 4.10: Compare test and deploy high-level view for both systems system development/maintenance; they will still contribute to the realization of other SO goals through the internal projects they share with other SO.

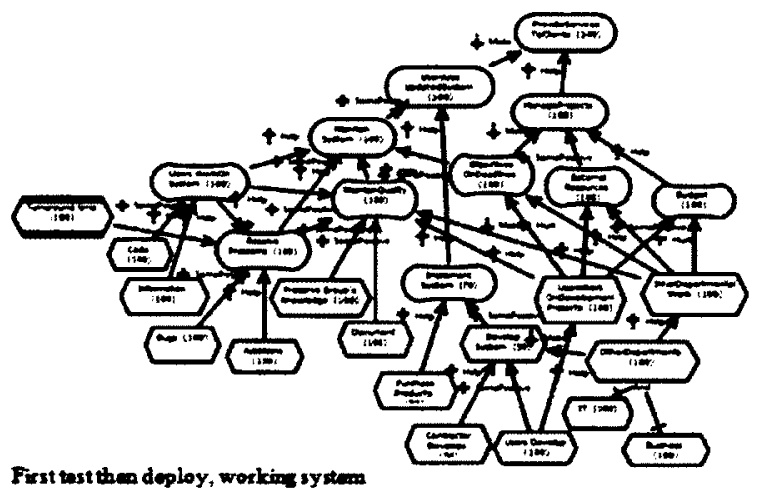

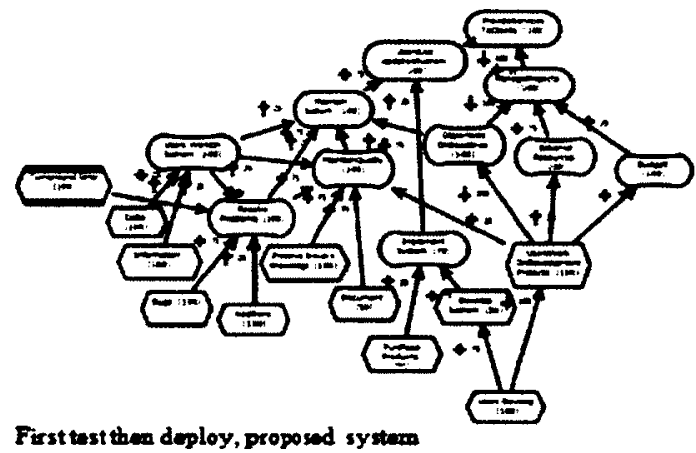

First test then deploy, proposed systom

Figure 4.11: Compare test and deploy detailed graph for both system

Next we analyze a few use case maps significant for the maintenance of the system, from development up to testing and deployment. The first map from Figure 4.12 presents the 


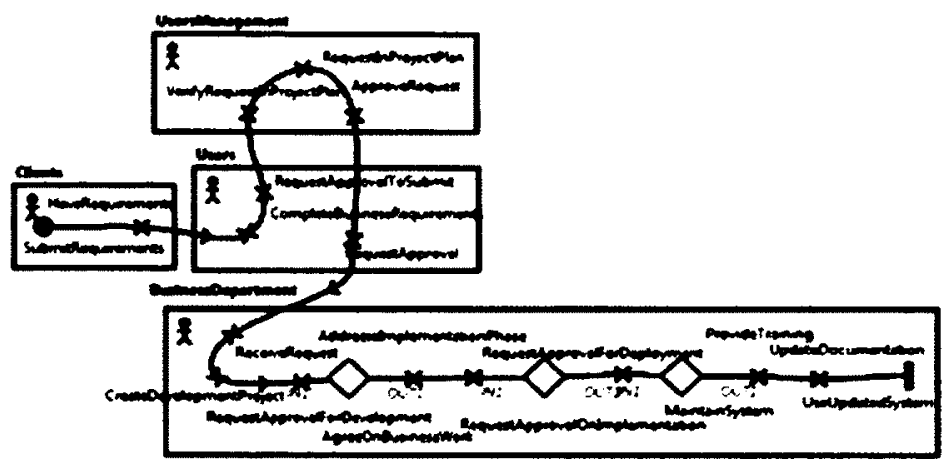

First test then deploy, overview UCM, working system

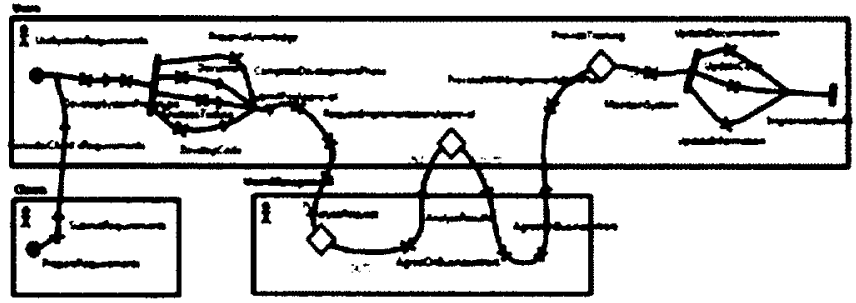

First test then deploy, overview UCM, proposed system

Figure 4.12: Compare high-level test and deploy maps high-level view of the way in which the $B D$ handles additions and corrections required by the Users work in the working system. The main activities stored in the stubs are located with BD while the Users have only few responsibilities.

As seen in the second

map in Figure 4.12, the analysis and approval of the requests has been transferred to the UM by the related stub in the proposed system, while the implementation, testing, deployment and potential training provided to other Users or clients will be done by the Users. If we drill down through the Request for Approval (the first stub that appears in both diagrams, for the working system under $\mathrm{BD}$, and for the proposed system under UM) we can access two more plug-ins.

While the approval responsibilities in the working system map would have been accomplished by BD with BDM and ITM support (and eventually the Contractor, if this work would be a valid option (see Figure 4.13) in the proposed system the process would be managed by the UM with approvals required from Stakeholders (see Figure 4.14). 


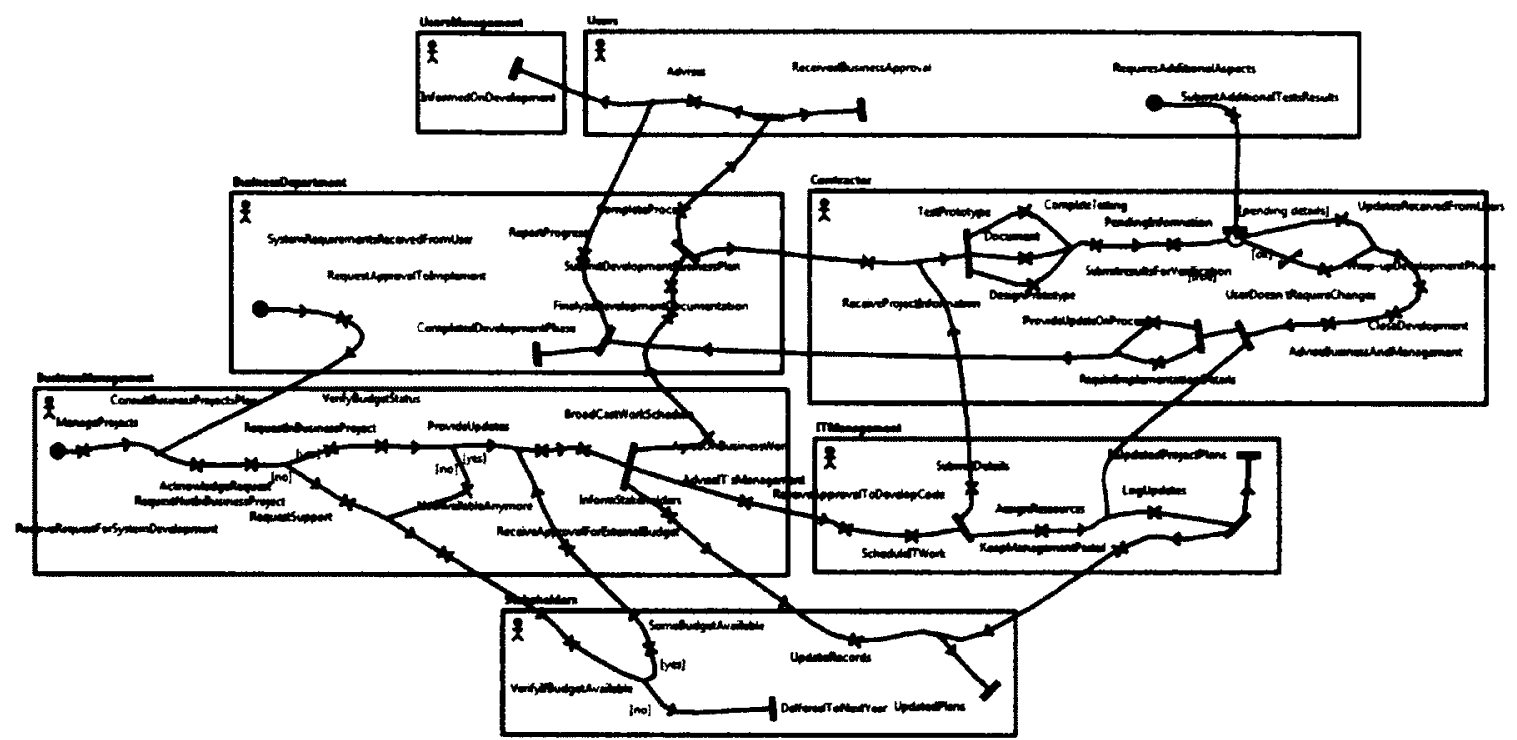

Figure 4.13: Request for approval process in the working system

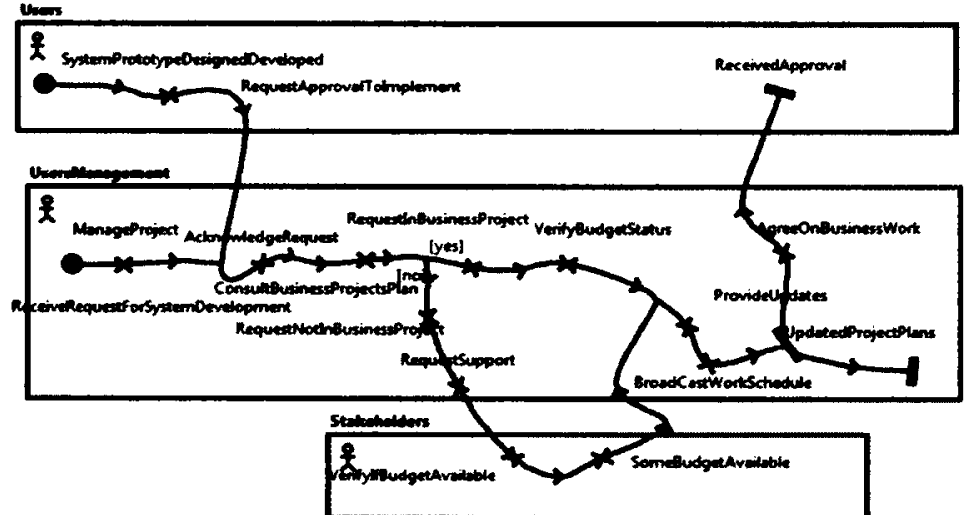

Figure 4.14: Request for approval process in the proposed system

\section{d) System monitoring}

The high-level diagram of the proposed system has been obtained from the working system by extracting the Actors and goals that are related to Users activities and work. The high-level graph realized for the proposed system in (Figure 4.15.b) and the detailed graph in Figure 4.16. 


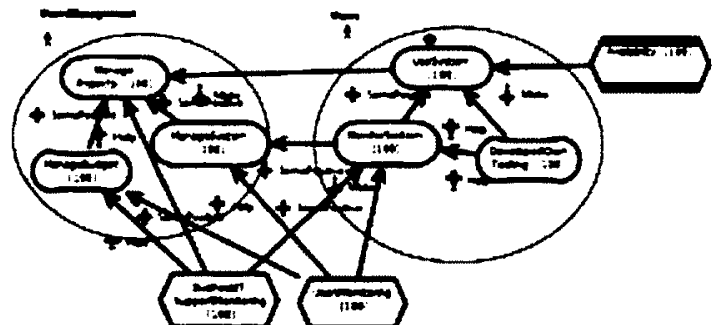

a. System monitoring, working system

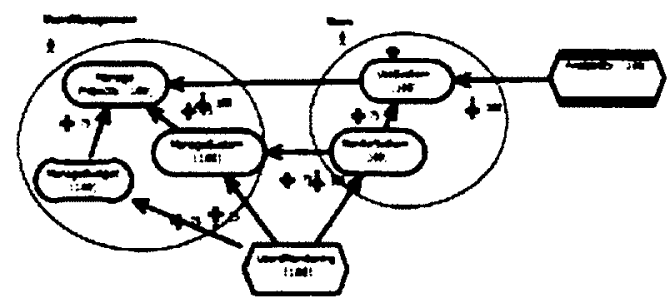

b. System monitoring proposed system

Figure 4.15: Compare system monitoring high-level graph in the two systems

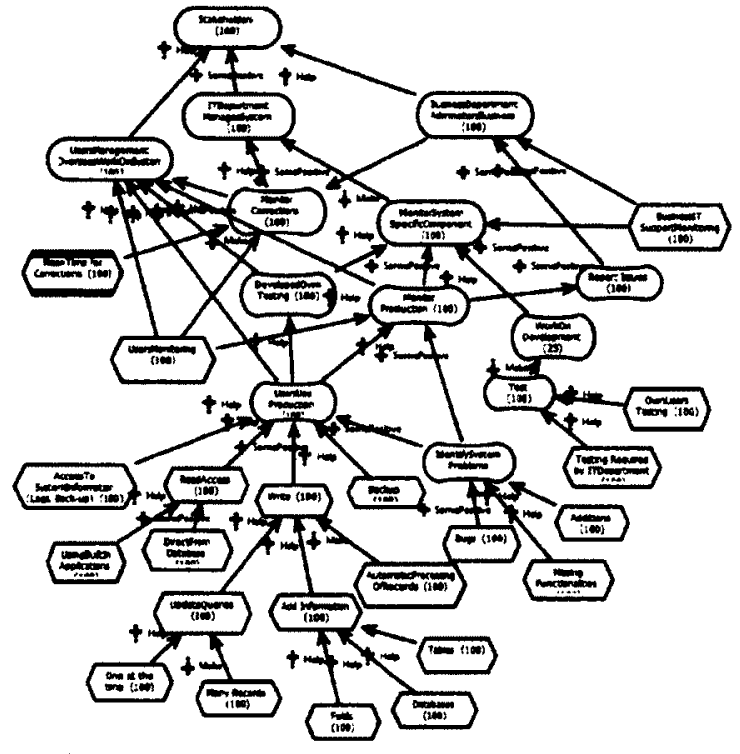

a. System monitoring, working system

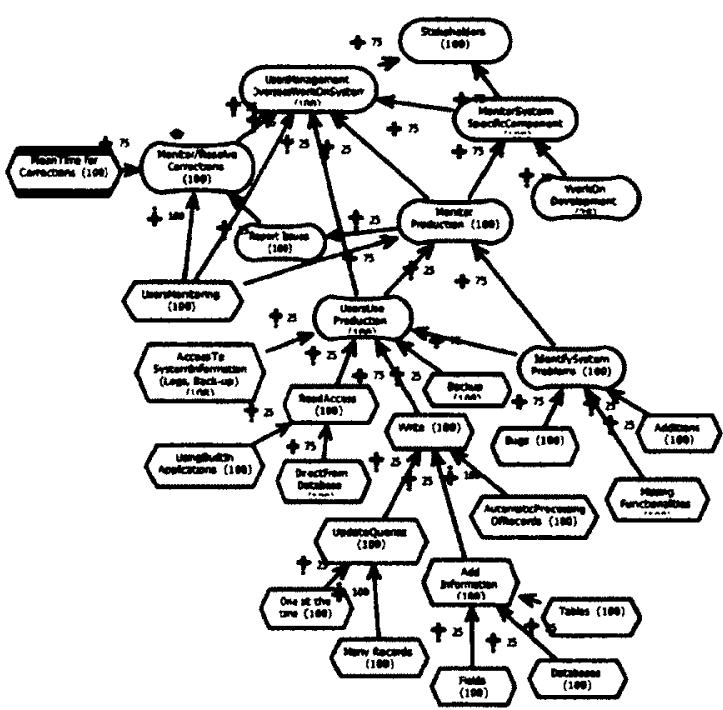

b. System monitoring, proposed system

Figure 4.16: Compare detailed system monitoring graph for both systems

In Figure 4.17 we compare the Users limited monitoring capabilities in the working system. GRL diagrams also realized for the proposed system in (Figure 4.16.b) do not contain anymore the tasks and goals related to BD and ITD's management of monitoring with the monitoring capabilities the Users have on the proposed system. 


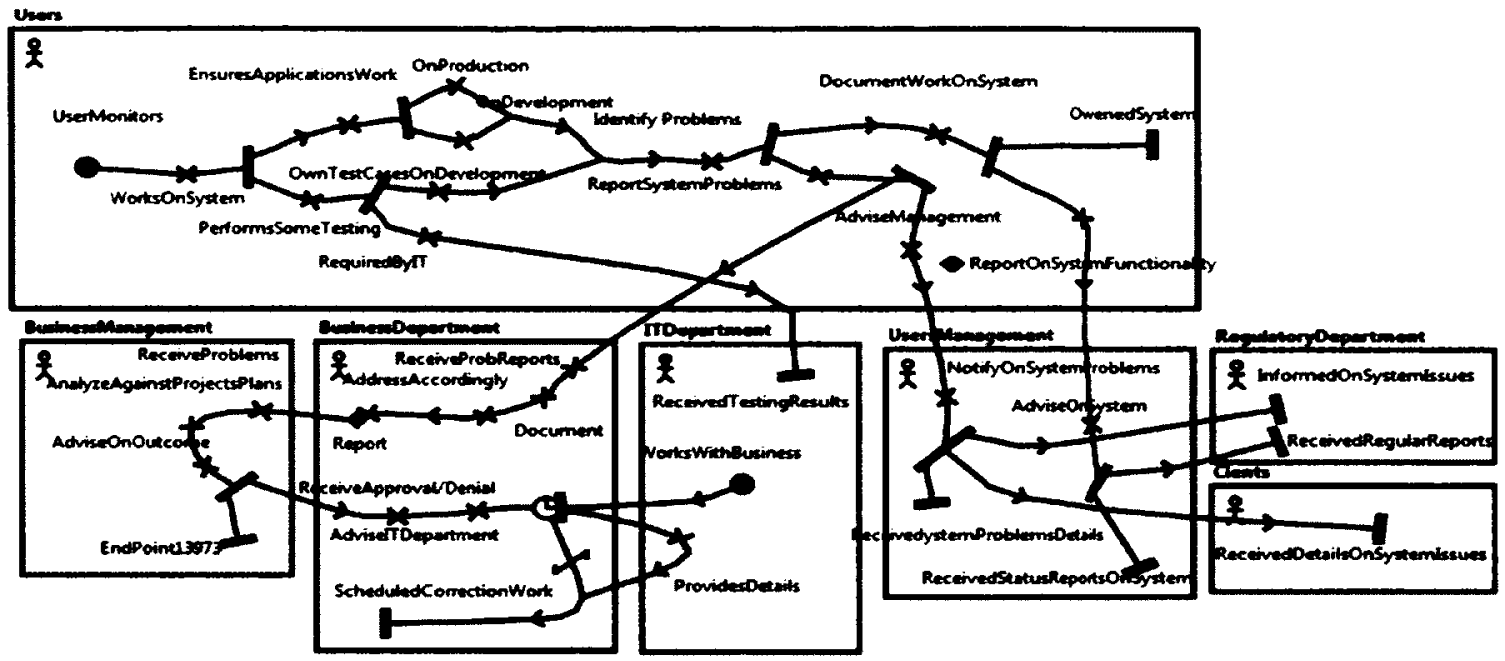

a. Users monitoring capabilities in the working system

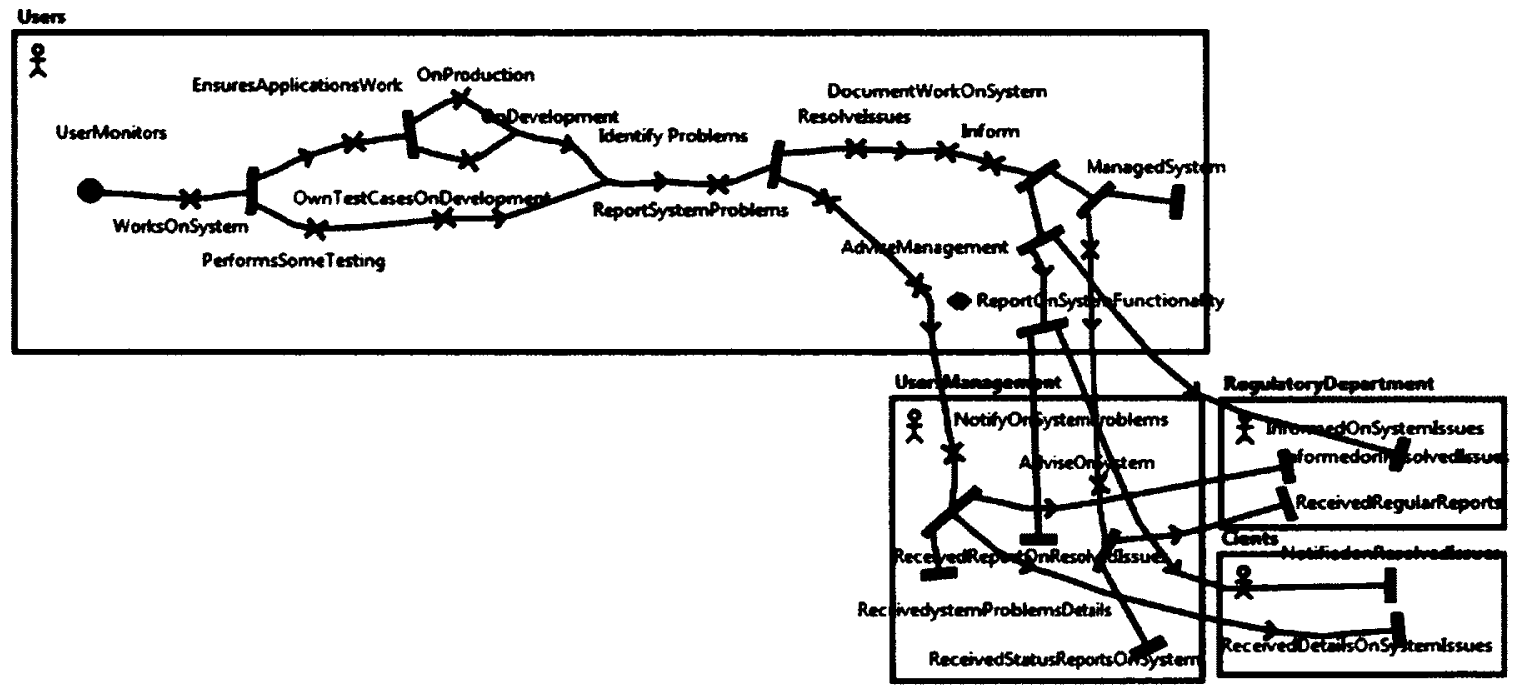

b. Users monitoring capabilities in the proposed system

Figure 4.17: Compare Users monitoring capabilities on both systems

In the next chapter we will compares the results of the evaluation of the two models. 


\section{Chapter: Performance Evaluation}

After designing the model and defining the performance indicators, we can execute the model and get the results. "A color code feed-back is displayed during the execution of evaluation, using red for denied and green for satisfied goals "[Amyot $11 \mathrm{~b}]$. We want to approach two objectives: compare the results with the demanded values, and produce feed-back according to the results of the evaluation. What happens if the evaluations are not revealing the results we expected to see; should we "look into changing characteristics, goals, components" or into redesigning the model? [Siddiqui02] After each evaluation, the results will be used to determine the satisfaction level of the goals, the realization of performance and business aspects. They are contributing factors in refining the goals and redesigning the business process if needed.

We will model and analyze two system cases: the Users system, known as working system, had recently completed a refurbishment process, and changed from an outdated technology to new equipment and software applications; and is currently managed by ITD and $\mathrm{BD}$; and the proposed system. This is another version of the working system, in which the Users and UM have administrative rights and responsibilities on their system. Once the model is created, we will proceed with its execution, to find out the impact of goals and their realization. For both system options we will have a look at the Users activities previously introduced in Chapter 3.

\subsection{Evaluation of use cases}

The results of each strategy evaluation are presented in a few ways: first they are displayed on the GRL graph, right after each evaluation, in a lable located above each 
intentional element; second, they can be exported from jUCMNav using the 'Export' tool in a .pdf report, known as jUCMNav Report or in a .csv report, in a Comma Separated Values format, that can be arranged and further exported in Excel to facilitate other types of operations. The .pdf reports we have generated vary from 40 to 112 pages, while one of the .csv reports has collected distinct information in more then 400 rows. Giving the numerous intentional elements we used to model different characteristics of the system, we decided to display in the next tables the evaluation results obtained for the goals of importance for the Users. The results are presented in association with each strategy, allowing us to compare the results between different strategies and also evaluate the global effect of a strategy in the realization of all goals.

During the analysis phase we are considering the four main categories of use cases related to the Users information system (as per description in Chapter 3):

(a) Users work flow and activities, takes a look at the way the Users work on the system

(b) Administrative information propagation and report, presents how information is propagated in the system across SOs, through various notifications and approvals

(c) First test then deploy, describes characteristics of the Users work, which is related to system maintenance, and often might require additions to the system or handling of issues (d) System monitoring, is in a way a wish list of monitoring capabilities for the Users.

The analysis of the system was done in Chapter 3, and the results are presented here.

\subsubsection{Users workflow and activities}

We will evaluate the GRL graphs per different strategies and analyze the results of the evaluation. "The concept of GRL strategies is a mechanism developed in jUCMNav to 
analyze GRL models using a set of user-defined evaluations"[jUCMNav12]. The evaluations "of GRL graphs show the impact of qualitative decisions on high level softgoals" [Amyot04]. As mentioned before, the GRL graph is composed of Actors internal and external to the organization, such as Users, UM, BD, BDM, ITD (with two components ITDSG and ITDNG), LD/RD, Clients and Stakeholders, as seen in Chapter 3 ; of intentional elements, tasks, softgoals and goals that contribute to the realization of the Users Actor and of the Users objectives; and of contribution links, which could Break or Help, Hurt or Make possible the realization of the element located at the end of the pointing arrow. Actors are "active entities that execute actions to achieve their goals"[Amyot03]. "A goal is an objective or concern used to discover and evaluate functional and non-functional requirements; softgoals are fuzzy goals" [Amyot03] and tasks are "solution which achieves goals or satisfies softgoals" [Amyot04]. All Actors interact with the Users in inter-departmental or cross organizations projects, as regulators or legislative bodies, upper management or clients; Each Actor is characterized by main goals, which also have an impact on realizing the Users objectives.

The intentional elements significant for the Actors are presented in Table 5.1. The strategies schema has been implemented to facilitate the analysis of GRL models by utilizing evaluations built on various characteristics, including system requirements, design choices, user needs, management participation, business priorities.

GRL strategies will use these characteristics to analyze and compare their effects on the goal satisfaction level. To proceed with the evaluation, we select the availability or "the satisfaction value [Amyot02c] to low-level intentional elements" [Roy07] of interest, chosen between $(-100)$ and $(+100)$. New strategies or strategies that have no evaluations 
set by the user, will generate 0 evaluations for actors, intentional elements. Therefore, to be able to evaluate a strategy, we have to go through each input element and set its particular characteristics in jUCMNav.

Table 5.1: Presentation of Intentional Elements per each Actor

\begin{tabular}{|l|l|}
\hline BD & Pans \\
\hline BD & $\begin{array}{l}\text { MaintainDocumentation, ManageProcesses, MonitorProjects, Use ITBusiness } \\
\text { Resources }\end{array}$ \\
\hline BDManagement & $\begin{array}{l}\text { Manage BusinessIT Projects, Manage Expenses, Manage Organization's System and } \\
\text { Applications }\end{array}$ \\
\hline Clients & Provide Data, Use System Model for Work \\
\hline ITD & $\begin{array}{l}\text { DeployUpgraded System, Impose Requirements, Manage Code, ResolveSystem } \\
\text { Problems }\end{array}$ \\
\hline ITDManagement & Complete Corrections,Manage IT Projects, MinimizeWorkload, Use Budget \\
\hline LD/RD & Respect Legal Aspects \\
\hline Users & $\begin{array}{l}\text { Application Requirements, CompleteWork on System Assist in Identifying/Resolving } \\
\text { Issues, Manage Projects, Report on Activities, Add Enhancements, Handle Bugs, Test } \\
\text { Prototype, Software, Hardware, Define Projects, Manage Projects, Set Requirements, } \\
\text { Define Project Plan, Work with other departments }\end{array}$ \\
\hline $\begin{array}{l}\text { Users } \\
\text { Management }\end{array}$ & $\begin{array}{l}\text { Administer Budget, Keep Spendings Low, Manage Group Projects, Manage } \\
\text { Objectives, Use Min Staff }\end{array}$ \\
\hline
\end{tabular}

Then we selected the propagation algorithm, quantitative GRL algorithm, which will use the value corresponding to their realizations per strategy, to determine the realization of the elements and the satisfaction level reached by each of them [Roy07] [Pengfei07].The evaluation result for a goal is calculated only during the evaluation process, and will be displayed on the top of the goal, in accordance with the selected algorithm. In our case, the satisfaction level is a numerical value, which has a color code associated with it. For an Actor, the evaluation shows its global satisfaction, and it is based on the characteristics of the intentional elements in relation with that Actor. Color red corresponds to satisfaction level 'denied' (-100); green corresponds to Satisficed $(+100)$; while yellow corresponds to the default value, set to 0 , [Roy07]. The evaluation range used for our case 
study is $[-100,100]$, one of the evaluation ranges provided by jUCMNav. "This implies that " $(-100)$ " is the worst evaluation" value or denied and $(+100)$ "is the best" [jUCMNav12] evaluation result or satisficed. By convention, the other evaluation levels correspond to the following satisfaction levels: $(+100)$ for Satisficed, (from +1 to +99$)$ is Weakly Satisficed, (0) for Unknown, (from -1 to -99$)$ is Weakly Denied, and (-100) for Denied. There are two more distinct evaluation levels used aside the evaluation range: the evaluation level of (-101) which corresponds to qualitative evaluation of Conflict, and the evaluation level of (-102) which corresponds to qualitative evaluation of Unknown.

Table 5.2 Input / Output Elements

\begin{tabular}{|c|c|c|}
\hline BD & $\begin{array}{l}\text { MaintainDocumentation, Use } \\
\text { ITBusiness Resources }\end{array}$ & ManageProcesses, MonitorProjects \\
\hline BDManagement & Manage Expenses & $\begin{array}{l}\text { Manage Organization's System and } \\
\text { Applications Manage BusinessIT Projects }\end{array}$ \\
\hline Clients & & Provide Data, Use System Model for Work \\
\hline ITD & Impose Requirements, Manage Code & $\begin{array}{l}\text { Deploy Upgraded System, ResolveSystem } \\
\text { Problems }\end{array}$ \\
\hline ITDManagement & Use Budget & $\begin{array}{l}\text { Complete Corrections,Manage IT Projects, } \\
\text { MinimizeWorkload }\end{array}$ \\
\hline LD/RD & Respect Legal Aspects & \\
\hline Users & $\begin{array}{l}\text { Add Enhancements, Handle Bugs, Test } \\
\text { Prototype, Software, Hardware, } \\
\text { Security, Define Projects, Define } \\
\text { Project Plan, Work with other } \\
\text { Departments }\end{array}$ & $\begin{array}{l}\text { Application Requirements, Complete Work } \\
\text { on System, Assist in Identifying / } \\
\text { Resolving Issues, Manage Projects, Report } \\
\text { on Activities, Set Requirements }\end{array}$ \\
\hline $\begin{array}{l}\text { Users } \\
\text { Management }\end{array}$ & Keep Spendings Low, Use Min Staff & $\begin{array}{l}\text { Administer Budget, Manage Group } \\
\text { Projects, Manage Objectives }\end{array}$ \\
\hline
\end{tabular}

The Input / Output table has been built from the Users point of view, with the Users understanding of activities and processes running in the system. Each strategy is built on the same components, Actors, contributions, and has been inspired from real situations. Strategy (1.1) also known as WorkingSystem_ProcessesNotInPlaceYet This corresponds to an early evaluation in the development process, when the role of 
each SO, and participants Actors, are not defined yet, except the LD. (LD is the Legal Department, which works with the Regulatory Department; they always have a defined role as they impose legislations and regulations for the users system, which are mandatory and should be always respected as such). The qualitative evaluation of the LD has been set to weekly satisficed for a value of $(+10)$. The evaluation value of this goal will impact directly the evaluation and realization of Users Application Requirements, and further, of Requirements. Usually we would have expected to have the Users Application requirements satisfied at a higher level, given that mandatory aspects are part of Users requirements. However there are situations when updates of legal or regulatory information are in the works and it is not clear yet for the Users if such updates will have an impact on their system requirements until they receive notification from the legal and regulatory SO. Given that the inter-departmental work has not been defined or finalized yet, task Work with OtherDepartments has been evaluated to Unknown (with evaluation value -102). Due to same circumstances, it is unclear for the BD if they will be in charge with management of business administrative information, therefore two more tasks, Maintain Documentation will, be evaluated to Unknown (value -102); this will make difficult for BD to manage processes and Monitor the projects. The situation continues to be unclear for ITD, as well, for which it is unknown if they will Manage the Code for Users system and applications. For the Users it is unknown if bugs could be resolved, while any additions to the system have been put on hold for a while, indicated by the qualitative evaluation of weakly denied. The Security task has been set to weakly satisficed for an evaluation level of $(+25)$ indicating that the users system is secure, and also hosted in a secure environment. The Users still try do define their projects even 
though at a lower satisfaction level of $(+10)$. .In such conditions it is difficult for the Users to Complete Work on the system, to Provide Data to Clients, to Assist in Identifying Issues and to Manage Projects. Let us assume that we are also at the beginning of the new fiscal year, when the budgets and their cuts are not available yet. This situation makes difficult for UM to estimate if they could complete their projects with the staff available and if they could keep the spendings low, without knowing yet the projects that have been approved and the available budget. This is why we see the two tasks from the UM Actor, Keep Spendings Low and Use Min Staff being set to qualitative evaluation Unknown with evaluation level (-102) (as indicated in Figure 5.1 for Strategy (1.1) and in the table with evaluation results Table 5.3).

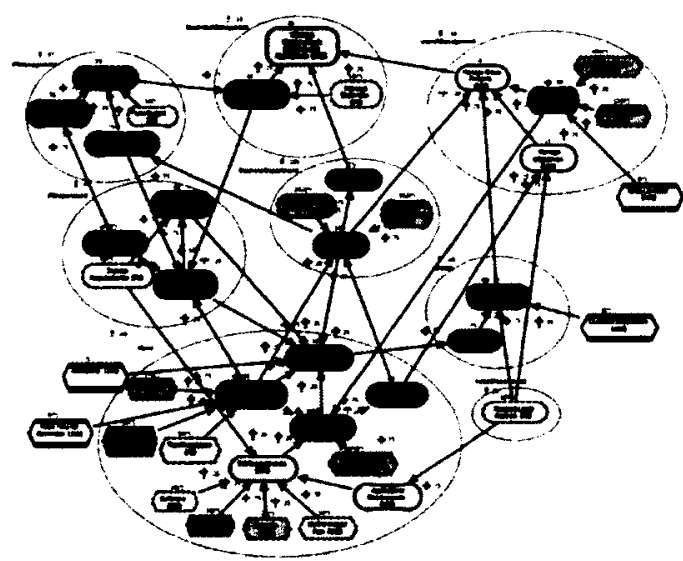

Figure 5.1: Evaluation of Strategy (1.1)

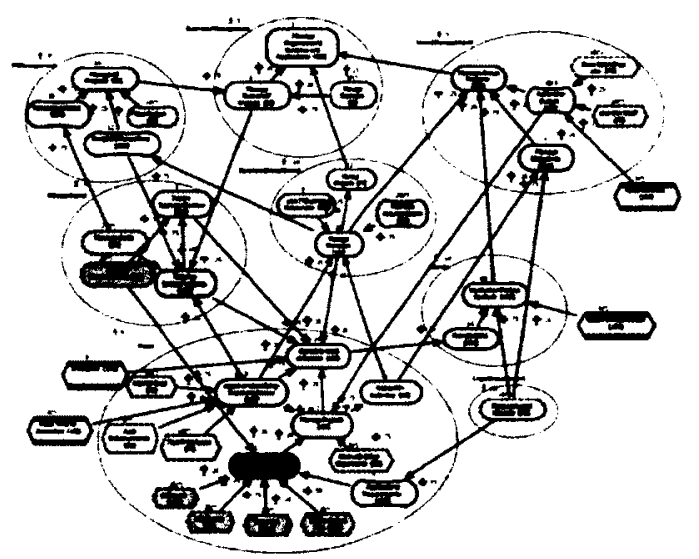

Figure 5.2: Evaluation of Strategy (1.2)

They impact the way the UM administers the budget, and the its corresponding goal Administer Budget, evaluated now as Weakly Denied (with evaluation level -50). In conclusion, according to the summary of evaluation results corresponding to Strategy (1.1) (out of 35 pages of evaluation results generated in the jUCMNav pdf report) we can see that the realization of most of the goals has been denied, therefore we don't consider this a satisfactory strategy that is beneficial to realizing goals. 
Strategy (1.2) also known as WorkingSystem_ChallengingTo WorkAcrossDepartments. This corresponds to a moment in the development cycle when activities at a higher organizational level have indicated common effort in trying to see if the role of each SO, and the way the SO interact can be defined. We have learned that some work arrangements at inter-departmental level have been discussed, yet with no concrete finalization at that time, and so task Work with OtherDepayments has been evaluated at (-25). Rather than being undefined, the inter-departmental work is now Weakly Denied but with a low value of $(-25)$. We maintained the same evaluation for the LD of $(+10)$ ,indicating a low acceptable value for the corresponding goal, as in Strategy (1.1) and for the same considerations. The evaluation value of this goal will impact directly the evaluation and realization of Users Application Requirements, and further, of Requirements. Due to same circumstances, the BD is not assigning yet IT and BD resources, so the corresponding goal is set to None. Until further details, the BD is not aware if to accept and maintain documentation from other SOs, therefore the corresponding goals has been set to a low value of $(-25)$; this will still make difficult for BD to successfully manage processes and monitor the projects. A light change can be seen for the ITD as well; they know they are in charge with requirements for the organization system and they have to provide guidance to SO; this is why impose requirements has been set to $(+25)$ for a weakly satisfied level. Until further details, ITD are not aware if they must accept correction requests or maintain the code, therefore Manage Code has been set to a low value of $(-25)$ corresponding to weakly denied. If we have a quick look at the higher level, we will understand that updates are to be provided soon; as in real situations, the management is first to receive an overview of the budget, 
which is reflected by both budget components from BDM and ITDM set to low weakly satisfied values of $(+10)$, as not all the details have been made available. This will make difficult to resolve problems and update changes on the system, and will have an impact on the realization of the Users' goals. This means for the Users that they could at least identify and handle low level bugs, eventually with some support from the network group, yet with no support from the solution centre group. This helps the value of Handle bugs to a Weakly Satisfied value of $(+10)$. Not knowing if there will be support in the inter-departmental work and projects, the Users interest in knowing what is happening with the system has been raised. The Users have knowledge on security, hardware and software, set now to Weakly Satisfied values of $(+25)$. The fact that ITD has contributed with requirements on the organization's system and network, help the Users to better understand their own requirements, reflected now by a calculated value of $(+45)$. Even though their work is still impacted by the lack of a decision on inter-departmental work, some progress has been reflected by the evaluation results for Manage Projects, now of (1) (in comparison with the previous value of $(-100)$ ), and by the evaluation results for Complete work on the system, with a value of $(-19)$ (considerably up from $(-100)$ in the previous case). The result for Report on Activities is evaluated now to (0) considerably up from a value of $(-87)$ in the previous case. Given the system's status and changes expected to happen, it is challenging for the users not only to work on the system, but also to provide assistance and work with Clients in general. This is indicated by the evaluation results for both Client's goals, Provide Data and Use System Module for Work; they have been both weakly denied at very low values of $(-14)$ and $(-8)$, respectively. Some changes have been noticed at the UM level as well. Even though the 
budget is still not available, let us assume that it is known that no more staff hiring is planned for the year. Thus, the available staff has to be assigned to projects, as indicated in task Use Min Staff, which is now set to weakly satisfied $(+10)$, as details are still needed to plan the work accordingly. Given that the budget is not available yet, will deny the spendings through task Keep Spendings Low. The evaluation of the Actors indicates a better satisfaction than in the previous case. Even though just for a few Actors, the evaluation results indicate low weakly satisfaction, at (+3) for UM, BM, (+9) for Users, (+10) for LD, while Weakly Denied for the others, including Clients, ITD, ITDM. In the light of the current estimates, the evaluation of the Graph is presented in Figure 5.2.

In conclusion, according to the summary of evaluation results corresponding to Strategy (1.2) we can see that there are still goals with realization denied, even though at lower levels then before. Also we can see that for four out of eight Actors, the realization is also weakly denied. Therefore we don't consider (1.2) a satisfactory strategy that is beneficial to realizing goals and Actors at SO and organization levels.

\section{Strategy (1.3) WorkingSystem_ProcessesInPlaceYet UnsureOn InterDepartmentalWork}

This case corresponds to a situation where information on own operations and projects is available to each SO and at organizational level; yet the budget might be known, but not available. (Usually, the budget becomes available at the end of the first quarter of the new financial year, so it takes time to have it delivered to each SO). This allows each SO to complete the definition of projects objectives, assign staff and budget for all internal affairs, even though we know that the budget will be Weakly Satisfied at a lower value $(+10)$ then other tasks and goals, including Define Projects, Handle Bugs, Impose Requirements, Manage Code of $(+25)$ or like Security of $(+50)$. Details on inter- 
departmental projects still require work, like sharing of resources, financial contributions, scheduling of deliverables, etc., shown as a Weakly Satisfied task of $(+10)$, now that the staff limitations are known and the budget is still to be distributed. We notified improvement in the realization of the goals in comparison with the results obtained in the previous two cases: the Users should work with a better system than before, given the realization of Set Requirements goal of $(+79)$, Manage of Projects $(+43)$, providing Users assistance in identifying/resolving the problems $(+26)$. The management of the Code will be done by IT and is set now to weak satisfaction of $(+25)$, with maintenance of documentation by BD still timidly at $(+10)$. The Users can work well with the system, indicated by the realization of goal Complete work on system, which is currently satisfied at $(+75)$; IT is deploying changes well as indicated by the realization of their goal at a weak satisfaction value of $(+47)$ and Resolve Problems at (+39). The goals of the clients are also realized well at $(+56)$ for Provide Data and $(+54)$ for Use System Module. The representative goals from the other Actors, as well as the Actors themselves, have been also Weakly Satisfied with positive results indicated in the two tables, and there are no more tasks, goals and Actors that have denied or weakly denied satisfaction levels. With these results presented, we can have a look at the evaluation of the Graph Fig.5.3, and conclude that this is a good strategy to start with. In order to appreciate how good the results are, or what are the results to aim for, each SO should look at the realization of their goals and define how much satisfaction is needed for certain objectives and projects. Similar discussions should be held at inter-departmental level, for projects, and at organizational level, for tuning up the work and the objectives. 


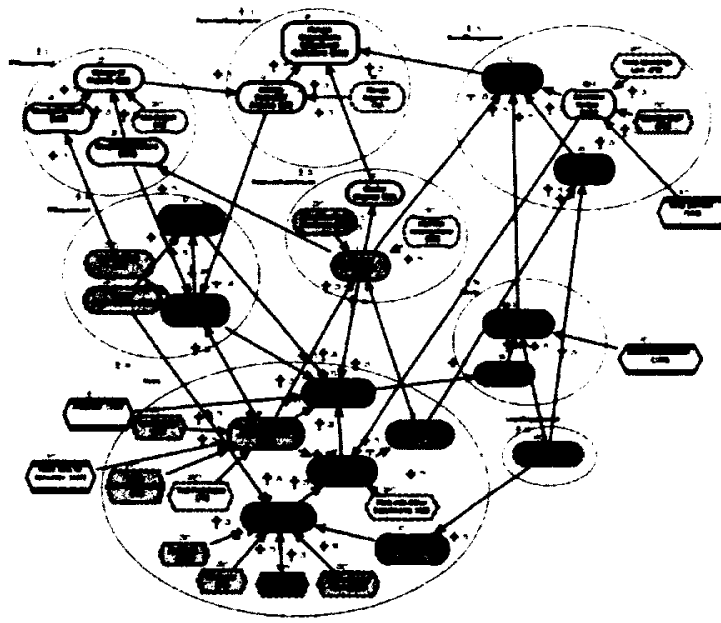

Figure 5.3:Evaluation of Strategy (1.3)

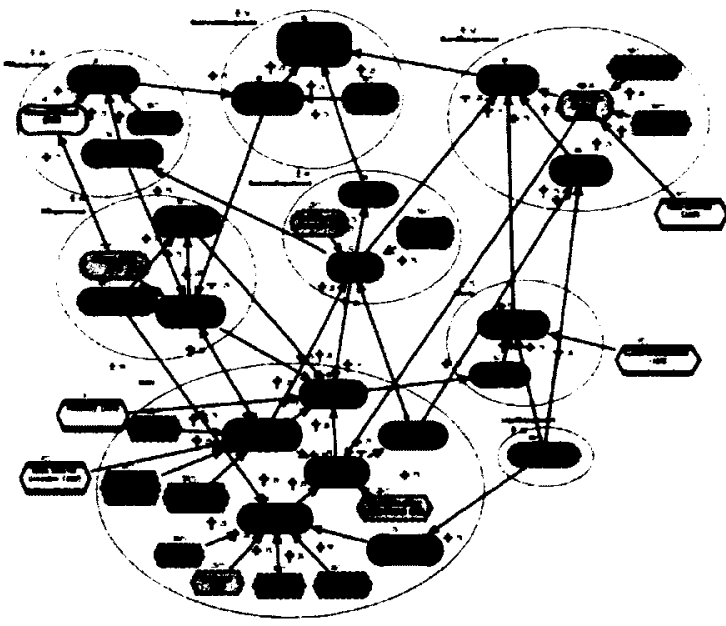

Figure 5.4:Evaluation of Strategy (1.4)

\section{(1.4) Strategy: WorkingSystem Pendingdetails OnWork AcrossDepartments}

This case indicates interest in inter-departmental projects by having the corresponding task set to weak satisfaction of $(+25)$. This is also reflecting in the settings of the other elements, including BD's Maintaining of Documentation set to $(+50)$, very good System Requirements realized at $(+90)$, ITD's Resolve System Problem goal realized at $(+71)$. ITD's goal Manage Code is still set to a weak satisfaction of $(+25)$ because the developers have to be trained in working with different systems, and often they change departments, taking the knowledge with them. We have also noticed that all goals have been realized with a weak satisfaction level, with a value above $(+17)$. Also all Actors have been satisfied at values greater than $(+38)$, with $(+59)$ for Users, $(+71)$ for Clients. Overall we conclude that this is the best strategy of all four strategies presented.

\section{(1.5) Strategy: WorkingSystem_StrategyWill Meet All Departments Objectives}

This strategy presents the best case scenario, in which the SO are supportive in the interdepartmental projects, providing assistance in timely manner, having staff available to 


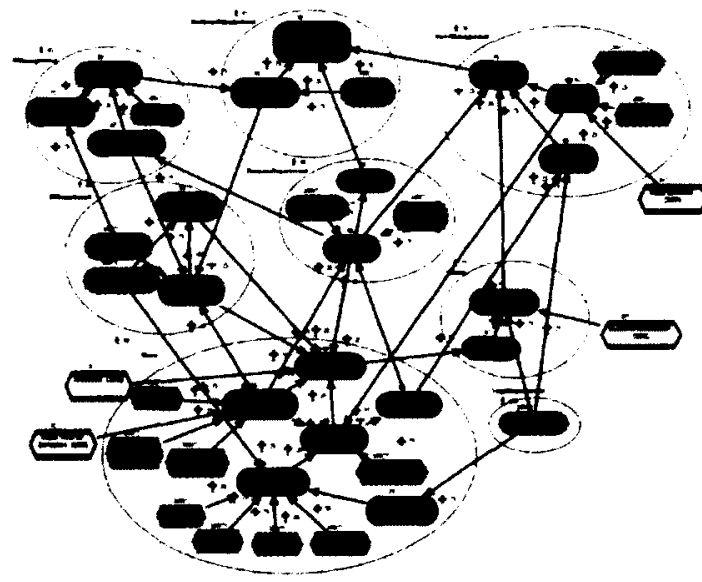

work on projects as required, sharing information, having a good communication inside the SO, as well across the SO's boundaries at the enterprise level. According to the evaluation results presented in tables below, we can conclude that this is the best strategy from all five presented for this case.

Figure 5.5: Evaluation of Strategy (1.5)

Table 5.3: Evaluation of main Users goals, working system

\begin{tabular}{|c|c|c|c|c|c|}
\hline (x) & 3ro, & 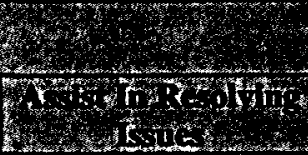 & 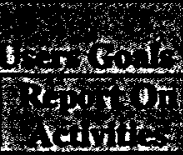 & Mrites & and \\
\hline 1.1 & 11 & -86 & -75 & -100 & -100 \\
\hline 1.2 & 45 & 9 & 0 & -1 & -19 \\
\hline 1.3 & 79 & 26 & 32 & 43 & 75 \\
\hline 1.4 & 90 & 61 & $\overline{59}$ & 79 & 90 \\
\hline 1.5 & 90 & 90 & 67 & 90 & 90 \\
\hline
\end{tabular}

Table.5.4: Evaluation of goals and Actors, working system

\begin{tabular}{|c|c|c|c|c|c|c|c|}
\hline (2) & 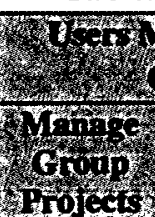 & Whing & tortist & ory & $\frac{1}{4}$ & Mrgits & rortctis \\
\hline 1.1 & -8 & $\begin{array}{c}-50 \\
\end{array}$ & -75 & -54 & -46 & -35 & -61 \\
\hline 1.2 & 7 & 0 & -14 & -8 & 9 & 3 & -10 \\
\hline 1.3 & 42 & 8 & 56 & 54 & 36 & 25 & 54 \\
\hline 1.4 & 69 & 24 & 67 & 73 & 59 & 52 & 71 \\
\hline 1.5 & 75 & 50 & 67 & 75 & 93 & 70 & 72 \\
\hline
\end{tabular}

Case: Users workflow and activities, working system, with KPI

The model has been enhanced with KPI. The role of KPI is to reflect "critical success measures" and "measure progress toward goals" [Wolf10]. The usual audience for KPI is the "senior management", which can get in only a few minutes updated information on significant processes [Wolf10], while the KPI's benefit is that "what gets measured, gets 
done" [Wolf10]. We have selected four KPIs to work with in this model: Customer Satisfaction, Keep Cost Low, Mean Time for Correction and Availability.

- Customer Satisfaction - to describe it we used the number of complaints received by the Users SO' Clients per year. The goal of the Users is to maintain the complaints around 3 per year; with worst case, of 12 issues due to various matters.

- Keep Cost Low - Let us look at few budget values that are required in the setting of the KPI: $\$ 500,000.00$ budget for the year to Users SO for all projects; $\$ 250,000.00$ assigned by the Users for maintenance/refurbishment of the system; $\$ 25,000.00$ to cover other matters. For the evaluation characteristic we estimated the values based o real work experience, according with each strategy and specific role and responsibilities of each actor in the process.

- Mean Time for Correction - represents the average time needed to resolve issues, component, application or module that failed or are not available. The correction period of time excludes any maintenance work. It is calculated as the sum of correction times divided by the sum of the number of corrective actions completed in one year. Due to today's performant technologies, we set the Target value to $24 \mathrm{~h}$, set Threshold to $72 \mathrm{~h}$, Down time to 3 days/year, and Worst value of the system down time was set to 5 days.

- Availability - is defined as the capability of the users to connect and work on the System, calculated as the system's working time (uptime expressed in percentage) per year. Given today's technology, the potential uptime could be 365 days, considering that back-up servers would automatically switch and take over in case of system failure. In reality, we have estimated that three days in total might be required by IT work on the system. In such a case, the Availability would be $(365-3) /[(365-3)+3]=362 / 365$, with 
result 0.991780821917 rounded for a final result value of $99.178 \%$. Let us have a look at few possible scenarios: the system was down for five days, an application had updated incomplete queries used for a month (31days); changes have been deployed on an old platform rather than on the system, which let us say it could have created issues for 41 days in a row, which would have caused an availability of: $(365-3) /[(365-3)+41]=$ $362 /(362+41)=362 / 403=0.89826302729$ with result approximated to $\sim 89.8263 \%$. Any time an intentional element with an assigned KPI gets selected from a GRL graph, its corresponding evaluation result will be presented through the KPI view [jUCMNav12] (Figure 5.8) indicating the performance overview of the component, one for each strategy. After evaluating this model for each of the five strategies, we analyzed the evaluation results, presented in an extract in the tables below. The results can be also exported in a pdf report or .csv, as earlier mentioned in Chapter 3 and Chapter 4.

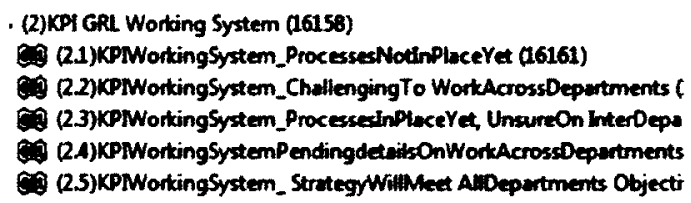

Figure 5.6: KPI strategies/working system

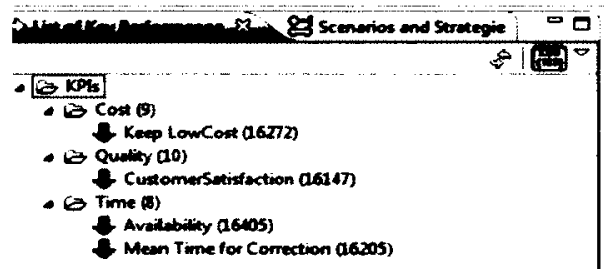

Figure 5.8: KPI Evaluation Strategy (2.1)

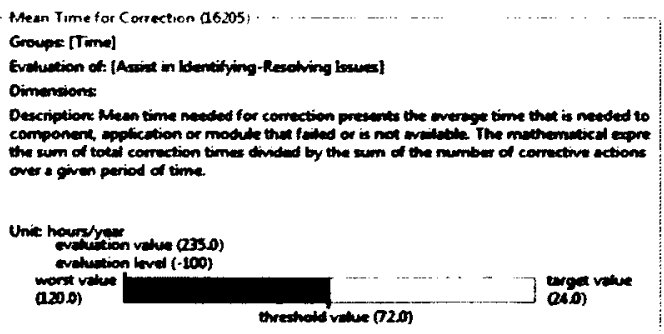

Figure 5.9 KPI Results for MTC

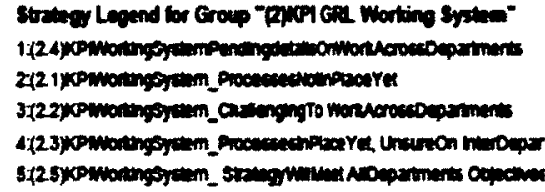

Figure5.7: KPI Strategies, extract from pdf report

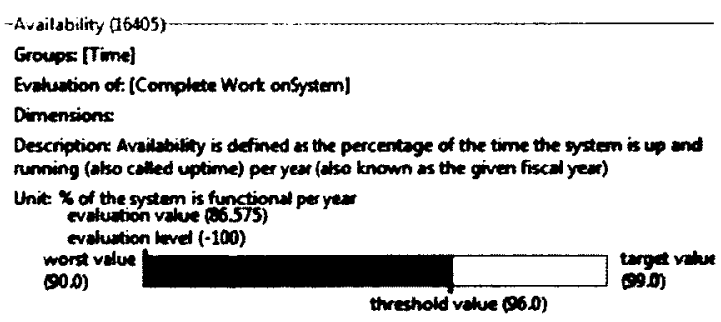

Figure 5.10 KPI Results for Availability 

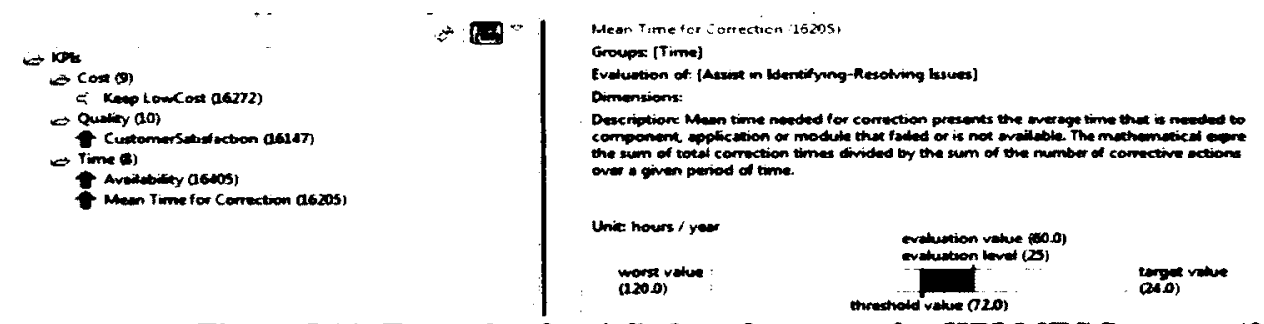

Figure 5.11: Example of satisfied performance for KPI MTC Strategy (2.3)

The values of the KPI results are also displayed in colors, similar with the color scheme used to indicate the realization of goals, and are also shown in a special window [jUCMav12] in (Figure 5.11). For each evaluation strategy chosen, (in our case for few examples) the results of the KPI evaluation are indicated in the following figures through symbols and three colors," in which the red down arrow means poor performance (Figure 5.11), the green-up arrow means acceptable performance (Figure next page) and the yellow arrow means the performance just meets the threshold value" [jUCMNav12].

Table 5.5: KPI Evaluation of Users goals, working system

\begin{tabular}{|c|c|c|c|c|c|}
\hline (6) & 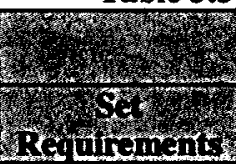 & 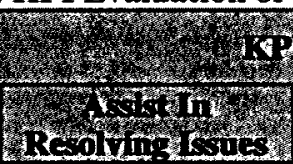 & 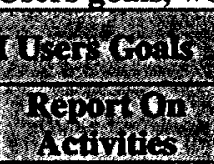 & 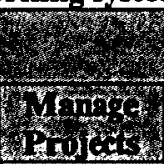 & 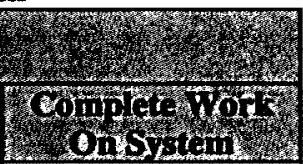 \\
\hline 2.1 & 15 & -100 & -75 & -100 & -100 \\
\hline 2.2 & 45 & -7 & -9 & 12 & -57 \\
\hline 2.3 & 79 & 32 & 36 & 48 & 90 \\
\hline 2.4 & 90 & 70 & 63 & 84 & 90 \\
\hline 2.5 & 90 & 90 & 67 & 90 & 90 \\
\hline
\end{tabular}

Table 5.6: KPI Evaluation of goals and actors, working system

\begin{tabular}{|c|c|c|c|c|c|c|c|}
\hline (6) & 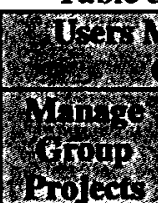 & 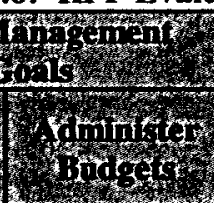 & 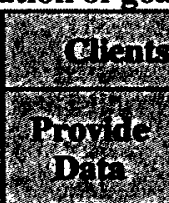 & 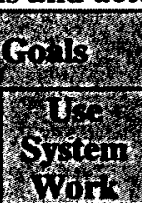 & $\frac{1}{4} x^{2}$ & 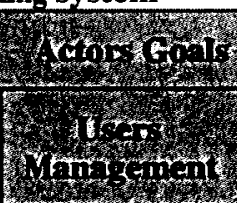 & 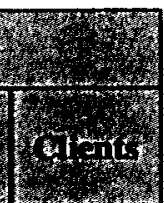 \\
\hline 2.1 & -13 & -53 & -75 & -68 & -47 & -39 & -70 \\
\hline 2.2 & -1 & 0 & -42 & -36 & 3 & 1 & -38 \\
\hline 2.3 & 47 & 8 & 67 & $\overline{72}$ & 38 & 26 & 70 \\
\hline 2.4 & 76 & 34 & 67 & 90 & 61 & 61 & 82 \\
\hline 2.5 & $\overline{85}$ & 74 & 67 & 90 & 93 & 90 & 82 \\
\hline
\end{tabular}


Table 5.7: KPI evaluation for Mean Time and Ability, working system

\begin{tabular}{|c|c|c|c|c|c|c|}
\hline 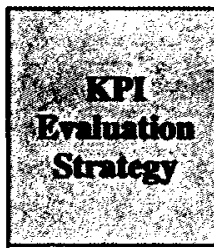 & 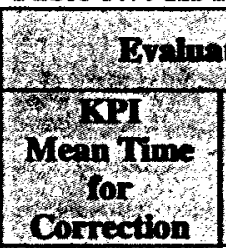 & 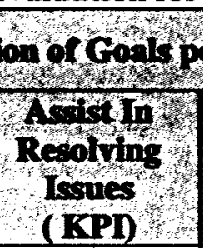 & 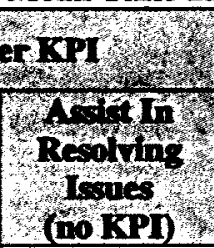 & 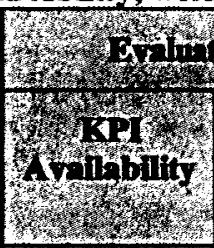 & 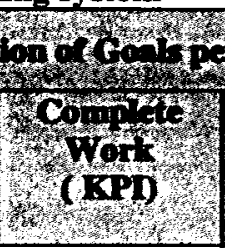 & 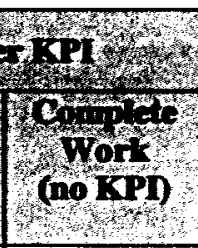 \\
\hline 2.1 & W61006 & -100 & -85 & 168701 & -100 & -100 \\
\hline 2.2 & 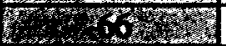 & -7 & 9 & W & -57 & -19 \\
\hline 2.3 & 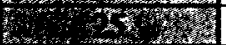 & 32 & 26 & $6, y, y_{0}$ & 90 & 75 \\
\hline 2.4 & 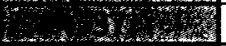 & 70 & 61 & 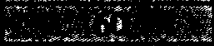 & 90 & 90 \\
\hline 2.5 & 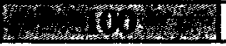 & 90 & 90 & 11 & 90 & 90 \\
\hline
\end{tabular}

Table 5.8: KPI evaluation for Keep Cost Low and Customer Satisfaction, working system

\begin{tabular}{|c|c|c|c|c|c|c|}
\hline 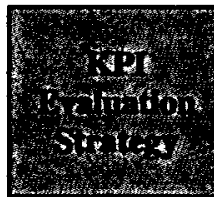 & 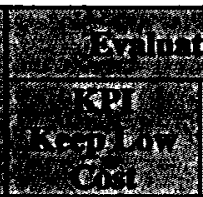 & $\begin{array}{l}\text { Administer } \\
\text { Budget } \\
\text { (KPI) }\end{array}$ & 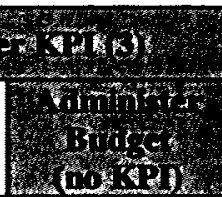 & 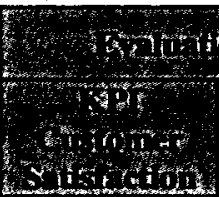 & 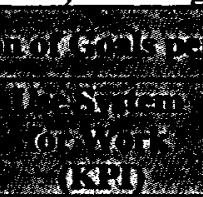 & $\begin{array}{l}9+(1) \\
6 \\
(0)\end{array}$ \\
\hline 2.1 & b 1 & -53 & -50 & 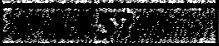 & -68 & -54 \\
\hline 2.2 & (b) & 0 & 0 & 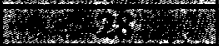 & -36 & -8 \\
\hline 2.3 & 18 & $\overline{8}$ & 8 & S & 72 & 54 \\
\hline 2.4 & 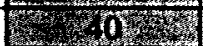 & 34 & 24 & W $=-70$ & 90 & 73 \\
\hline 2.5 & 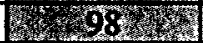 & 74 & 50 & -100 & 90 & 75 \\
\hline
\end{tabular}

For analysis we have compared the results obtained in the previous case, from the evaluation with no KPI, for certain goals and actors; with the KPI evaluation results, obtain in the four tables, corresponding to the same goals and Actors. We can conclude that the evaluation results obtained in the KPI model are at least equal (for some goals) or have better values for three strategies (2.3), (2.4), (2.5) which correspond to the best three strategies identified in the previous case; we can also conclude that the satisfactory results are located in the mid to second half of the weakly satisfied level.

\section{Case: Users workflow and activities, proposed system, with and without KPI}

The proposed system has been modeled from the working system, as presented in Chapter 4 , by extracting the intentional elements responsibilities and activities from the 
other departments performing business and internet technology services for the users system, and transferring them over the Users SO. The model obtain contains fewer Actors, given that the Users system is not dependent anymore on BD and ITD. The Users have the best interest to manage the system according to its mandate and requirements. Both strategies are very close to each other, as the Users will perform the same activities, including management of documentation, updating of operational business activities, monitoring of the system and resolving of issues. They have owned and managed the old system for 35 years, starting with software maintenance and ending with 24 h monitoring, back-up and recovery. The same KPIs with the ones presented in the working system have been used for the proposed system, and extracts of the evaluation results are presented in the tables and diagrams that follow.

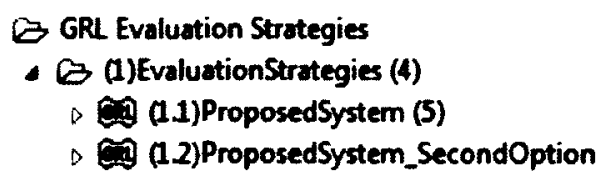

Figure 5.12: Strategies list 
Table 5.9: Evaluation of main Users goals (proposed system)

\begin{tabular}{|c|c|c|c|c|c|}
\hline Golmaton & 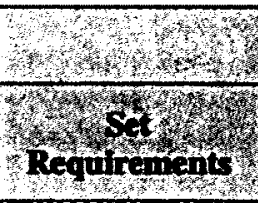 & 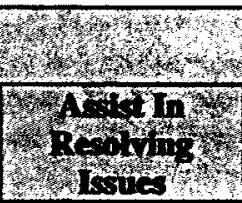 & 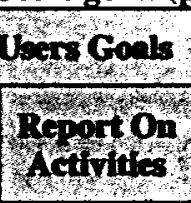 & 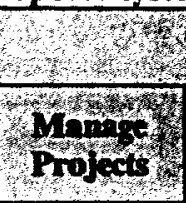 & W(x) \\
\hline 1.1 & 90 & 90 & 67 & 90 & 90 \\
\hline 1.2 & 90 & 100 & 67 & 90 & 100 \\
\hline
\end{tabular}

Table 5.10: KPI Evaluation of main Users goals

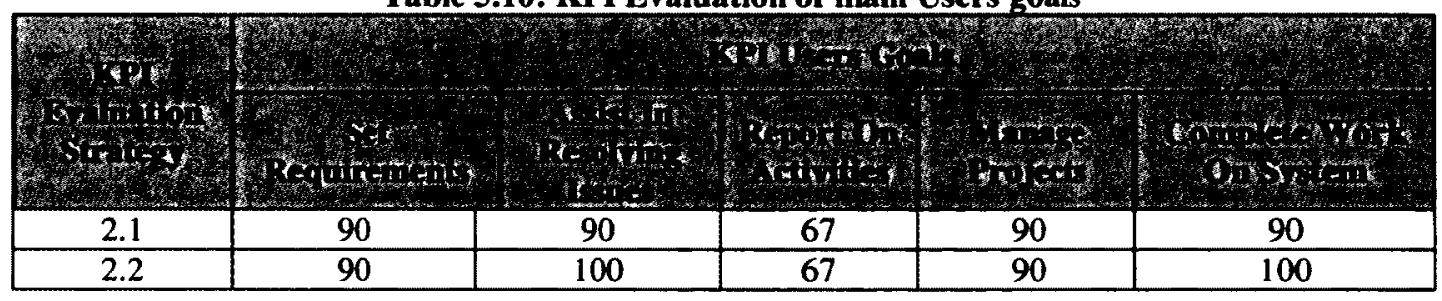

Table 5.11: KPI Evaluation of Users Management and Actors

\begin{tabular}{|c|c|c|c|c|c|c|c|}
\hline (1) & 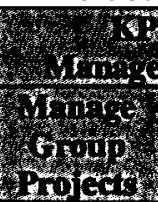 & 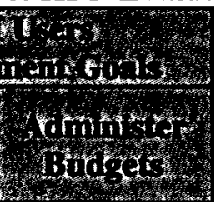 & 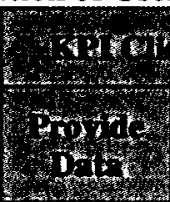 & 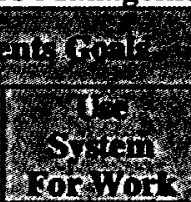 & $y_{1}$ & (x) & $y^{6}+15$ \\
\hline 2.1 & 90 & 45 & 67 & 90 & 65 & 73 & 82 \\
\hline 2.2 & 90 & 75 & 75 & 90 & 94 & 96 & 91 \\
\hline
\end{tabular}

Table 5.12: Evaluation of KPI 01 for Users main

\begin{tabular}{|c|c|c|c|c|c|}
\hline (4) & 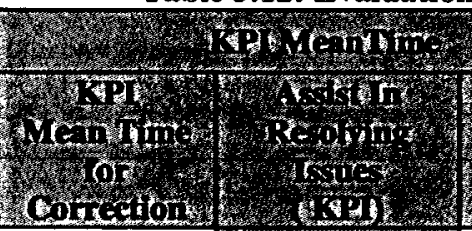 & 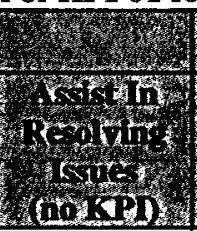 & (6) & 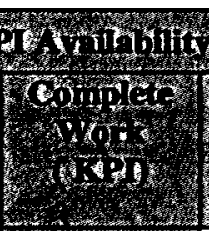 & 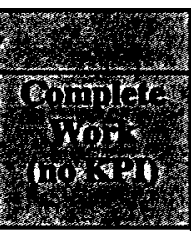 \\
\hline 2.1 & 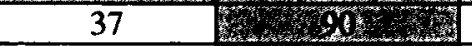 & 90 & 76 & W. & 90 \\
\hline 2.2 & monom & 100 & 100 & W 100 & 100 \\
\hline
\end{tabular}

Table 5.13: KPI Evaluation of Low Cost' and Customer Satisfaction

\begin{tabular}{|c|c|c|c|c|}
\hline (x) & 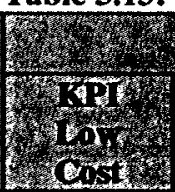 & 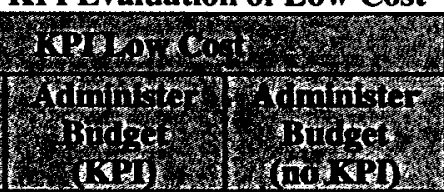 & 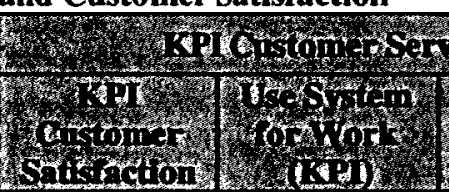 & $(6,5 \%)$ \\
\hline 2.1 & 36 & $\begin{array}{ll}36 \\
6\end{array}$ & 80 & 90 \\
\hline 2.2 & 100 & 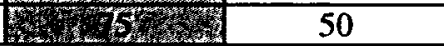 & 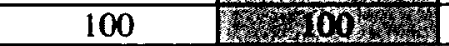 & 100 \\
\hline
\end{tabular}




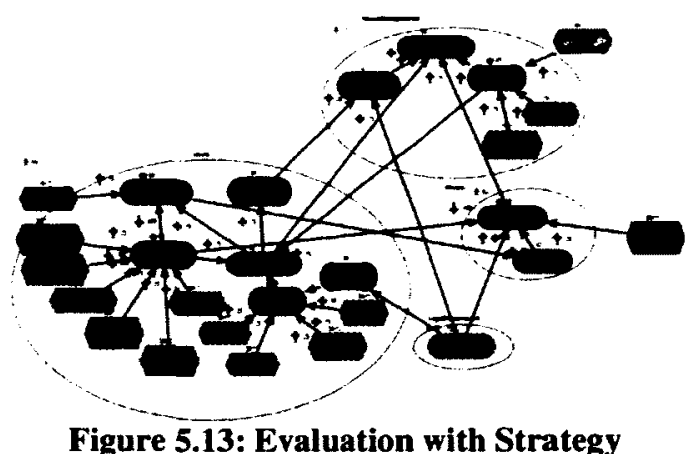

(2.1)

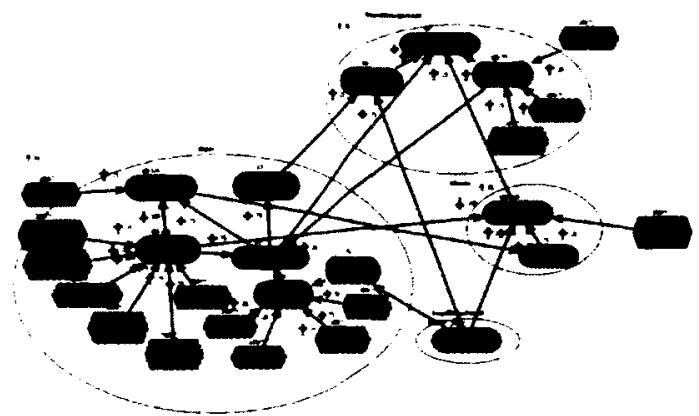

Figure 5.14:Evaluation with Strategy

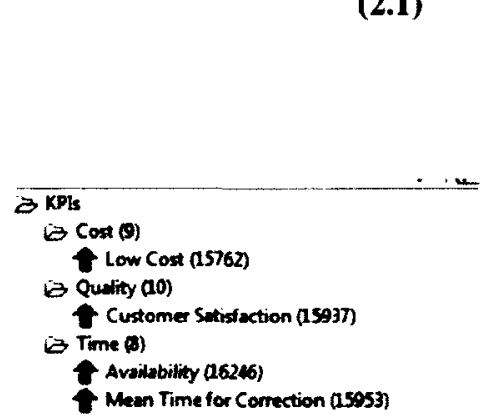

Figure 5.15: Example of KPI Results for Strategy 2.1

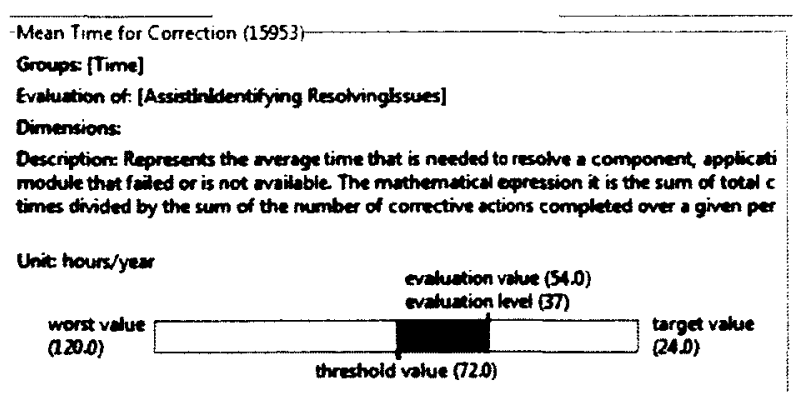

Figure 5.15. Example of KPI Results for Strategy 2.1

For the proposed system, all intentional elements and the Actors are realized, from mid weakly satisfied values to satisfied values of $(+100)$. There are slight differences between the evaluation with or without KPI. Also the results obtained here with the first strategy (2.1) (the worst case) can be situated above the results obtained from the evaluation of Strategy (1.4) and below the evaluation results corresponding to Strategy (1.5) from the working system case. Due to space limitation, we present the analysis results for the other cases in Appendix A, Appendix B and Appendix C. 


\section{Chapter: Conclusions}

We conclude in this chapter the approach of the thesis, by presenting a review of the thesis contributions and discussing future work and future possibilities of studies.

\subsection{Contributions Summary}

The contributions of the thesis are as follows:

1. We have developed the URN model of a complex distributed system, inspired from reality, with participants from different departments across a very large organization, which have different goals and responsibilities and operate with their own budget, managers and staff.

2. The URN model was used to help us understand and evaluate how goals of different actors can be achieved in this study. We have completed the following objectives:

- understood how administrative information is being monitored at system level;

- analyzed monitoring techniques, following the reporting path from the creation of the reporting objects to the organization's approval authorities, learning how various requests are addressed according to priorities (not only for the solicitor, but also for the different departments that are involved in the processing and resolution of a request);

- took into account operational planning of projects and routine activities (plans created one year in advance, by each sub-organization and at organizational level), budget received (usual lower with at least $10 \%$ from the estimated budget required), staffing and collaboration with other sub-organizations and organizations in inter-departmental and external projects; 
- learned how system issues are being reported to management, system administration and system users;

- followed from the Users perspective, the life cycle of a corrective action request.

3. In order to improve the efficiency of the working system, a new model known as the "proposed system" has been designed. It has been modeled from the original working system, by transferring responsibilities and activities to the Users from other suborganizations that were managing the users system and its operational business activities at organizational level, while being in charge with the maintenance and management of other tens of sub-organizations. The overhead introduced in reporting and processing of requests has been reduced in the proposed system, as the reporting process includes collaboration only between Users, Users' managers and Stakeholders, in most situations. There is no conflict of interest to be mitigated or conflicting objectives, as the Users and their own managers have the same interests regarding the work on the system, and the same objectives at sub-organization level. There is no delay in addressing and resolving the requests; the users sub-organization is in charge with the maintenance of the system, and they will handle the requests according to their own priority schema, as they have done during the 35 years they have owned the system. The knowledge in system development, implementation, deployments, monitoring and maintenance will be preserved inside of the suborganization that owns the system; this will allow the Users to also manage the operational business aspects, including maintenance of system requirements and any future system developments. While working with the proposed system, the Users are capable of providing customer and technical assistance 24 hours a day. 


\subsection{Lessons Learned}

The evaluation results without and with KPIs presented in Chapter 5 indicate a better realization of the goals for Users, UM and Clients actors. They also show an increase in the satisfaction level for the strategies in which the Users have more rights and responsibilities while working with the system, and this includes the strategies used in both the working and the proposed system. An important point is that the conclusions reached with the help of different evaluation strategies converged toward the same big picture. From modeling the system and analyzing the results, we have learned that there are various processes and activities related to the management of the system and operational business which are carried out by other departments and which can be improved. We believe that it is a good exercise for the departments that are currently managing different systems at organizational level to analyze what type of services are provided by the departments versus the distinct services and needs required by each suborganization. We believe that with the help of URN models, the departments in charge will be able to identify potential conflicting objectives, which goals could be realized and which couldn't, and what is the impact for the Users' sub-organization in terms of work quality, delivering time, work flexibility and cost. We believe that all sub-organizations should achieve their goals at the satisfaction level required by their mandate. Work relationships required by inter-departmental projects could also be looked at and improved in the future. Through our URN models, we have looked not only at the realization of the high level goals, but have also explored the satisfaction level of the actors that interact with other participants through their work contributions and goals. We also believe that the points of view characteristics to each sub-organization should be 
brought to the discussion table and presented clearly in relationship with their own set of goals. This should allow identifying how difficult (or easy) is to achieve different goals at different satisfaction levels, the reasons why that happens and what are the consequences of achieving the goals for each sub-organization.

Modeling in jUCMNav allows for a great visual presentation and evaluation of goals and strategy in front of the stakeholders. Similar with the idea of having a three minutes speech in the elevator with one of your executives, the tool allows for a brief presentation of system and project matters, allowing a quick depiction of the goals in relationship with the requirements, participants, options and strategies for realizing them. It is quite an advantage for system development, given that URN allows for early modeling and evaluation, before finalizing the design and starting the implementation plans. The modeling can be done at very little cost, and most important, prior to purchasing equipment and technology or to hiring contractors. It also provides testing capabilities in early design phases, allowing for changes and additions of elements, including goals and actors, in parallel with the revision of requirements. We believe that such modeling is beneficial, giving that in traditional system developments the use cases are revised mainly in the testing phase, while the testing is done after the system has been designed and developed, quite close to deployment. If the testing results indicate that the system does not meet its requirements, then various changes need to be done at different design and implementation phases, which require further testing and validation. We believe that in the case of early modeling and evaluation, this correction cycle is of a smaller magnitude, involving changes mostly at the model level, and allowing for the evaluation of different strategies without the expenses of the previous method. 
We have also learned that the centralization of operational business activities and technological services could be beneficial, as long as the work characteristics, objectives, processes and project types of each SO are understood and considered prior to the integration of the SOs into the activities supported and administered at the organizational level. The realization of goals at the planned satisfaction level should be investigated and evaluated at organizational level prior to transferring operational management responsibilities and ownership of the system from the Users to other departments. It is not only one SO at a time that must be managed and served at given parameters, we are looking at least at tens of distinct applications from different SOs running on the distributed system, with unique mandatory activities, different goals and various satisfaction levels of their objectives. We are looking at programs, milestones and deadlines that are manageable at the SO level, yet could create potential interferences of interests and objectives, and potential conflicting situations, once they are brought out at an organizational level without due consideration, testing, evaluation and analysis. The organization should be able to develop a few mitigation strategies that would look for the best solutions for realizing the objectives, giving equal opportunities to SOs to realize their own objectives in a timely manner. The organization should be able to investigate different ways of managing the system, including more participation from the users, and to create communications and inter-departmental work relationships at a new level.

All the documentation related to system development and maintenance, starting with operational business requirements documents, and up to use cases, test plans, code comments and users manuals should be available to users and all parties involved in the maintenance process at all times. These are living documents that must be periodically 
revised and updated along with the evolution of the system. Any changes in any areas, including business activities of the system, code fixes, software upgrades and updates, hardware maintenance, should be discussed a priori with the users' clients, to identify potential impacts, analyze solutions and agree / approve the final approach.

Last but not least, things need to done the right way as early and as much as possible. The modeling allows us to line up the steps to be completed during the development and maintenance of the process. It allows us to model the responsibilities in a sequential and logical order, to ensure that the steps will be completed along the development map. Missing steps on the way could generate difficulties along the development route, which may require timely and costly fixes, with impact on the quality of the system. We understand that the rapid development of internet technologies and software applications, the need of speed and the demand to quickly adapt operational business aspects to new requirements and client's demands, set the bar high in system developments and maintenance. Rather than considering these road blockers, we should look at the challenging aspects and conduct the development process to better meet the need for better systems and better applications.

\subsection{Limitations}

Model building. Given the circumstances in which the model has been designed and implemented, the model validation is based on expert knowledge rather than on measurements. During the model development, we had very limited access to the requirements documentation of the real-life system. 
Tool limitations. Ranking of strategies could cause issues in displaying the strategies after the report files are exported to pdf. For example, if the strategies are listed as $1,2,3$, 4 in the jucm model, after the export to the pdf report, their order is often changed. It is true that a legend is provided, but changing the ranking becomes a problem when you deal with different strategy groups composed of many different strategies. Another problem is that orphans elements appear to be captured in the model/in the reports.

\subsection{Future Work}

With respect to the evolution of the system, we would like to analyze in future work what level of details is sufficient to represent the system in the model, and to identify how much system knowledge must be transferred into goals and responsibilities in order to achieve a better estimation of the goals realization. This study is considering a large scale distributed system used by a large organization across its sub-organizations and departments. Many services are being shared at departmental level and various interdepartmental projects are being required in order to complete projects at each suborganization or department level, as well as at high-level. Therefore a future direction would be to investigate how performance can be achieved by each sub-organization, and what would be required at the modeling level to obtain a better realization of goals, and implicit an increased performance for each sub-organization. We would also like to look into finding a class of KPIs that could be applied to each sub-organization across the enterprise, so then we can compare the performance results. It would be also interesting to investigate how many KPIs would be enough for monitoring the system, and how many would be required to assure a very good monitoring, and to enable the fine tuning 
of the system at both model and realization levels. There are many cases in which the goals of a sub-organization are being ranked at lower levels than for other groups, without necessarily meaning that their objectives are less important that the objectives of another group. We also believe that future research work is required in the area of goals handling in the system, as well as in the model. Therefore we would like to find out if there are any mitigation techniques that would allow us a better realization of the goals, without having a negative impact on the completion of the goals from the other suborganizations, and on the objectives at organizational level.

Another future direction is to investigate the operational business aspects and processes, and their relationships with the system requirements and current functionalities. 


\section{References}

[Aalst03] van der Aalst, W.M.P., ter Hofstede, A.H.M. and Weske, M. (2003), "Business process management: a survey", Proceedings of the International Conference on Business Process Management, BPM 2003, Eindhoven, The Netherlands, 26-27 June

[Abrial74] Jean-Raymond Abrial: Data Semantics. IFIP Working Conference Data Base Management 1974: 1-60

[Abujudeh10] H Abujudeh, R.Kaewlai and all, "Quality Initiatives: Key Performance Indicators for Measuring and Improving Radiology Department Performance", Massachusetts General Hospital,Harvard Medical School, MA 02114, 2010

[Aderholz01] M. Aderholz,,K. Amako and The MONARC Collaboration, "Distributed applications monitoring at system and network level "Computer Physics Communications, Volume 140, Issues 1-2, 15 October 2001, Pages 219-225

[Alexander09] Alexander, Ian and Beus-Dukic, Ljerka. Discovering Requirements: How to Specify Products and Services. John Wiley, 2009

[Alsumait04] Alsumait, A.: User Interface Requirements Engineering: A Scenario-Based Framework.Ph.D. thesis, Concordia University, Canada, August 2004

[Amyot00] D. Amyot and G. Mussbacher, "On the Extension of UML with Use Case Maps Concepts." $<<U M L \gg>2000,3^{\text {rd }}$ International Conference on the Unified Modelling Langiuage, York, UK (October 2000), LNCS 1939, 1631

[Amyot01a] Daniel Amyot, Gunter Musssbacher "Minitutorial on the Use Case Maps (UCM) Notation", 2001

[Amyot01b] Daniel Amyot, Gunter Mussbacher, "Introduction to Use Case Maps", Bridging the Gap between Requirements and Design with Use Case Maps, 2001

[Amyot02a] Amyot, Daniel, "Using the User Requirements Notation", URN, ITU-T Workshop on the "Use of Description Techniques", November 23, 2002

[Amyot02b] Amyot, D., Mussbacher, G., and Mansurov, N.: Understanding Existing Software with Use Case Map Scenarios. 3rd SDL and MSC Workshop (SAM02), Aberystwyth, U.K. LNCS 2599, Springer, 124-140, June 2002

[Amyot02c] Amyot, D., and Mussbacher, G.: URN: Towards a New Standard for the Visual Description of Requirements. In: 3rd SDL and MSC Workshop (SAM02), Aberystwyth, U.K., June 2002. LNCS 2599, 21-37

[Amyot03a] Amyot, D., "Introduction to the user requirements notation: Learning by Example", Computer Networks: The International Journal of Computer and 
Telecommunications Networking, Vol. 42, No. 3, pp. 285-301, 2003

[Amyot03b] Amyot, Daniel; Mussbacher,Gunter, http://www.UseCaseMaps.org/pub

[Amyot03c] Amyot, Daniel; Mussbacher,Gunter; "Bridging the Requirements/Design Gap in Dynamic Systems with Use Case Maps (UCMs). (2003)

[Amyot 03d] Amyot, D. and Eberlein, A.: An Evaluation of Scenario Notations and Construction Approaches for Telecommunication Systems Development. In:

Telecommunications Systems Journal, 24:1, 61-94, September 2003

[Amyot03e] D.Amyot, "Introduction to the User Requirements Notation "CSRS'03

[Amyot04] Daniel Amyot "Introduction to the User Requirements Notation", ITU-T Q.18/17 Rapporteur, SITE, University of Ottawa, Canada, 2004

[Amyot05a] Amyot, D., Roy, J.-F., Weiss. M.: UCM-Driven Testing of Web Applications. Prinz A., Reed R., Reed J. (Eds) SDL 2005: Model Driven, LNCS 3530 , Springer, 247-264, June 2005

[Amyot05b] Amyot, D., Weiss, M., and Logrippo L.: UCM-Based Generation of Test Purposes.Computer Networks, 49(5), 643-660, December 2005

[Amyot07] D. Amyot, J.Kealey and all, "Short Tutorial on jUCMNav" in "Towards Integrated Tool Support for The User Requirements Notation", 2007

[Amyot08a] Daniel Amyot and Gunter Mussbacher, "Development of Telecommunications Standards and Services with the User Requirements Notation, 2008

[Amyot08b] Daniel Amyot, "ITU-T's User Requirements Notation (URN) and jUCMNav", CASCON Workshop, October 29, 2008

[Amyot09] Daniel Amyot, Jennifer Horkoff , Daniel Gross, and Gunter Mussbacher , “A Lightweight GRL Profile for i* Modeling”, 2009

[Amyot11a] D. Amyot and G. Mussbacher,User Requirements Notation: The First Ten Years, The Next Ten Years (Invited Paper), Journal of Software, Vol. 6, No.5,May 2011

[Amyot 1 1b] D. Amyot, G. Mussbacher, S. Ghanavati, J.Kealey, "GRL Modelling and Analysis with jUCMNAV", CEUR Proceedings of the $5^{\text {th }}$ International $i^{*}$ Workshop (iStar2011), 2011

[Amyot99a] Amyot, D.; Andrade, R.;Logrippo, et all; "Wireless Communications and Systems", Emerging Technologies Symposium, 1999 Page(s): 14.1 -14.7

[Amyot99b] D.Amyot and L.Logrippo,"Use Case Maps and LOTOS for the Prototyping 
and Validation of a Mobile Group Call System", Computer Communications, Special Issue on FDTs

[Andrade00] R. Andrade and L. Logrippo, "Reusability at the early Development Stages of the Mobile Wireless Communication Systems". Proceedings of the $4^{\text {th }}$ World Multiconference on Systems, Cybernetics and Informatics (SCI 2000), Vol. VII, Orlando, Florida, 2000, 11, 16

[Andrade01] R. Andrade, "Capture, reuse, and Validation of Requirements and Analysis Patterns for Mobile Systems”. Ph.D. thesis, SITE, Univ. of Ottawa, Canada, May 2001

[Armour10] Quintin Armour, Didier Thizy,"Developing Successful Healthcare Software: 10 Critical Lesson", Healthcare Solutions MAcademian 2010

[Bali03] B.Bali's, M. Bubak, W. Funika, R. Wismüller, "A monitoring system for multithreaded applications" Published by Elsevier Science B.V, Future Generation Computer Systems, Volume 19 (2003) Pages 641-650

[Bell76] Bell,T.,Thayer,T."Software Requirements: Are They Really a Problem”, Proc. ICSE-2:2 ${ }^{\text {nd }}$ International Conference on Software Engineering, San Francisco 1976 61-68

[Bertolini05] Bertolini, D., Novikau, A., Susi, A., and Perini, A. : TAOM4E : an Eclipse ready tool for Agent-Oriented Modeling. Issue on the development process. Technical Report, Automated Reasoning Systems Division, ITC-IRST, Italy, 2005

[Billard04] Billard, E.A.: Operating system scenarios as Use Case Maps. ACM Workshop on Software and Performance, 266-277, 2004

[Bochmann 10] Gregor v. Bochmann, "User Requirements Notation (URN), Based on Powerpoint slides by G. Mussbacher(2009) with material from D. Amyot, University of Ottawa, SEG3101 (Fall 2010)

[Boehm81] B.W.Boehm, Software Engineering Economics. Prentice Hall, 1981

[Boldt01] Boldt, L.:Trends in Requirements Engineering. In: People-Process Technology Technology Builders Inc., 2001

[Borgida09] A. Borgida, V. Chaudhri, P. Giorgini, E. Yu, “ Conceptual Modeling: Foundations and Applications: Essays in Honor of John Mylopoulos” Springer 2009

[BPMN06] Business Process Modeling Notation (BPMN) Specification Final Adopted Specification dtc/06-02-01 2006

[Brocke 10] vom Brocke, J. \& Rosemann, M., "Handbook on Business Process Management: Strategic Alignment, Governance, People and Culture (International Handbooks on Information Systems). Berlin: Springer 2010 
[Bubenko80] Bubenko, J.A.: Information Modeling in the Context of System Development.IFIP Congress. 395-411 (1980)

[Johannesson07] Johannesson, P.,"The Role of Business Models in Enterprise Modelling In J. Krogstie et al. Conceptual Modelling in Info. Systems Eng", 123-140. Springer 2007

[Cares08] Cares, C., Franch, X., et all : “iStarML: An XML-based Model Interchange Format" For i* in: Castro, J.B., Franch, X., Perini, A., Yu, E. (Eds.): Proc. 3rd Int. i* Workshop, Recife, Brazil, February 11-12, 2008. CEUR Workshop Proceedings 322 CEUR WS.org: 13-16 (2008)

[Chan00] M. Chan, Y. Lin and X. Wang, "A Scalable Monitoring Approach for Service Level Agreements Validation", Network Protocols, Proceedings. 2000 International Conference Osaka, Pages 37-48

[Chen76] [Peter Pin-Shan Chen, "The Entity-Relationship Model - Toward a Unified View of Data",Massachusetts Institute of Technology", ACM Transactions on Database Systems, Vol1, No1, March 1976, Pages 9-36

[Christe192] Christel, Michael and Kyo C. Kang (September 1992). "Issues in Requirements Elicitation". Technical Report CMU/SEI-92-TR-012. CMU / SEI. Retrieved January 14, 2012

[Chung00] L. Chung, B.A. Nixon, E. Yu and J. Mylopoulos, "Non-Functional Requirements in Software Engineering", Kluwer Academic Publishers, USA 2000

[Dahl66] Ole-Johan Dahl and AhlI Risten Nygaard, , "SIMULA an ALGOL-Based Simulation Language " , Communications of the ACM , Norwegian Computing Center, Oslo, Norway, Volume 9 / Number 9 / September, 1966

[Damian07] Daniela Damian “ Stakeholders in Global Requirements Engineering: Lessons Learned from Practice" University of Victoria, 0740- 7459/2007 IEEE

[Davies93] Davis, A. "Software Requirements: Objects, Functions and States", Prentice Hall,1993

[deBruin01] de Bruin, H. and van Vliet, H. "Scenario-Based Generation and Evaluation of Software Architectures". Generative and Component-Based Software Engineering (GCSE'01), LNCS 2186, (2001)

[Dingel09] Juergen Dingel, "Models in Software Development: methods, techniques, and tools”, Course Notes for CISC836 Fall 2010/11, School of Computing Queen's

University Kingston, Ontario, August 2009

[Dingel 11] Juergen Dingel, "Models in Software Development: methods, techniques, and tools", Course Notes for CISC836, Fall 2010/11 
[Dongmo09] Cyrille Dongmol and John A. van der Poll2, "Use Case Maps as an Aid in the Construction of a Formal Specification" 2009

[Easterbrook04] S. M. Easterbrook, "What is Requirements Engineering", 2004

[Eclipse 12] Eclipse Project Release Notes, Release 3.7.2, Last revised Feb 7, 2012 file:///C:/Program\%20Files\%20(x86)/eclipse/readme/readme eclipse.html

[Elahi07] Elahi, G., Yu, E.: A Goal Oriented Approach for Modeling and Analyzing Security Trade-Offs. ER 2007. Auckland, New Zealand. LNCS vol. 4801. 375-390. Springer (2007)

[Embley1 1] David W. Embley, Bernhard Thalheim, Handbook of Conceptual Modeling: Theory, Practice, and Research Challenges, 2011

[European96] European Software Institute, "European User Survey Analysis", Report USV_EUR 2.1, ESPITI Project, January 1996

[Garlan00] Garlan, D. "Software Architecture: A Roadmap", 2000

[Ghanavati07] 15 Ghanavati, S., Amyot D., and Peyton, L.: Towards a Framework for Tracking Legal Compliance in Healthcare. 19th Int. Conf. on Advanced Information Systems Engineering (CAiSE'07), Trondheim, Norway. LNCS 4495, Springer, 218-232, June 2007

[Greenspan82] Greenspan, S.J., Mylopoulos, J., Borgida, A.: Capturing More World Knowledge in the Requirements Specification. ACM/IEEE Int. Conf. Softw. Eng.: 225 235 (1982)

[Gro95] [Standish95] The Standish Group, "Chaos. Technical Report T23E-T10E”, The Standish Group, 1995

[Hamou-Lhadj05] Hamou-Lhadj, A., Braun, E., Amyot, D., Lethbridge, T.: Recovering Behavioral Design Models from Execution Traces.9th European Conference on Software Maintenance and Reengineering (CSMR), IEEE Computer Society, 112-121, March 2005

[Hassine05] Hassine, J., Rilling, J., and Dssouli, R.: An ASM Operational Semantics for Use Case Maps. 13th IEEE International Requirement Engineering Conference (RE05), IEEE CS Press, 467-468, September 2005

[Hassine07] Hassine, J., Rilling, J., a nd Dssouli, R.: Formal Verification of Use Case Maps with Real Time Extensions, 13th SDL Forum (SDL'07), Paris, France. LNCS 4745, Springer, 225-241, September 2007.. LNCS 4745, Springer, 225-241, September 2007

[Ho08] Chih-Wei Ho, Laurie Williams, Brian Robinson "Examining the Relationships between Performance Requirements and "Not a Problem" Defect Reports", 
Department of Computer Science, North Carolina State University, IEEE 2008

[Insfran02] E. Insfran, O. Pastor, R. Wieringa, "Requirements Engineering-Based Conceptual Modeling" 2002

[ITU96] ITU-T, Recommendation Z.120- Message Sequence Chart (MSC), 1996

[ITU02] ITU-T, Draft Recommendations Z.151 - Goal-oriented Requirements Language (GRL), Geneva 2002

[ITU03] ITU-T, Recommendation Z.150, User Requirements Notation (URN) Language Requirements and Framework, Geneva, 2003.

[ITU03a] ITU-T, URN Focus Group: Draft Rec. Z.151 - Goal-oriented Requirement Language (GRL). Geneva, Switzerland, Sept. 2003

[ITU08] International Telecommunications Union (ITU-T) Recommendation Z.151: User Requirements Notation (URN) - Language Definition. (2008)

[Jackson83] Jackson, M.: “System Development”, Prentice-Hall (1983)

[Jacque04] Jacques, R.: Web Applications Wide Open to Hackers. vnunet.com news, http://www.vnunet.com/News/1152521, Feb 5, 2004

[J-PRiM] J-PRiM. A Process Reengineering i* Modelling Tool, http://www.ideaciona.com/PhD/JPRIM/

[jUCMNava] jUCMNav. University of Ottawa.

http://jucmnav.softwareengineering.ca/jucmnav/

[jUCMNavb] jUCMNav Wiki,

http://jucmnav.softwareengineering.ca/twiki/bin/view/ProjetSEG/

[jUCMNAVc] Material documented by Jean Francoys Roy, 2006

http://jucmnav.softwareengineering.ca/ucm/bin/view/ProjetSEG/GrlDemol ?cover=print,

[jUCMNav11] jUCMNav Created by jenhork. Last Modification: Tuesday 19 of July, 2011, http://istar.rwth-aachen.de/tiki-index.php?page=jUCMNav

[jUCMnav12] jUCMNav Online Help Eclipse 2012

[Kealey05] Kealey, J., Tremblay, Amyot, D., et all, “jUCMNav: une nouvelle plateforme ouverte pour l'édition et l'analyse de modèles UCM. In : Nouvelles TEchnologies de la RÉpartition (NOTERE'05), Gatineau, Canada, August 2005, 215-222

[Kealey07] J.Kealey and D. Amyot, "Enhanced Use Case Map Traversal Semantics", 
2007 http://lotos.site.uottawa.ca/ucm/pub/UCM/VirLibSd107jUCMNav/SDL07-KealeyAmyot.pdf

[Kethers05] Kethers, S., Gans, G., Schmitz, D., Sier, D.: Modelling trust relationships in a healthcare network: Experiences with the TCD framework. In Bartmann D., et al. (eds.): European Conf. on Information Systems (ECIS). Regensburg, Germany. 1321 $1328(2005)$

[Kling96] Kling, R., Computerization and Controversy: Value Conflicts and Social Choices, 2nd edition, Morgan Kaufmann, (1996)

[Ko09] Ryan K. L. Ko," A computer scientist's introductory guide to business process management (BPM), ACM Crossroads 15(4), ACM Press 2009

[Kohlbacher09] Kohlbacher, M., "The Effects of Process Orientation on Customer Satisfaction, Product Quality and Time-Based Performance", Paper presented at the 29th International Conference of the Strategic Management Society, Washington DC, October $11-14,2009$

[Kotonya98] Kotonya G. and Sommerville, I., "Requirements Engineering: Processes and Techniques", Chichester, UK: John Wiley \& Sons. 1998

[Krechetov 06] Ivan Krechetov, Bedir Tekinerdogan, Alessandro Garcia, "Towards an Integrated Aspect-Oriented Modeling Approach for Software Architecture Design”, 2006

[Lamsweerde00] Lamsweerde, Axel van. Requirements Engineering in Year 2000 - A Research Perspective, in Proceeding of 22nd International Conference on Software Engineering(ICSE 2000), Limerick, ACM Press, 2000

[Lee97] Lee, A.Y. and Bodnar, B.L. , "Architecture and Performance Analysis of PacketBased Mobile Switching Center-to-Base Station Traffic Communications for TDMA". Bell Labs Journal. Summer 1997. 46-56

[Liskov 00]B. Liskov, J. Guttag, “ Program Development in Java”, AddisonWesley, 2000

[Liu01a] Lin Liu and Eric Yu, "From Requirements to Architectural Design - Using Goals and Scenarios", From Software Requirements To Architectures Workshop (STRAW 2001)

[Liu01b] Liu, L. et al. (2001) GRL and OME. http://www.cs.toronto.edu/km/GRL

[Liu03a] Liu, L., and Yu, E.: Designing Information Systems in Social Context: A Goal and Scenario Modeling Approach. In: Information Systems (Journal), Vol.29, No.2, 2003

[Liu03b] Liu, L., and Yu, E.: Security and Privacy Requirements Analysis within a Social 
Setting. In: International Conference on Requirements Engineering (RE'O3), Monterey, California, p. 151-161, September 2003

[Lockerbie06] Lockerbie, J.A., Maiden, N.A.M.: REDEPEND: Extending i* Modelling into Requirements Processes', IEEE Int. Conf. on Requirements Eng., 361-362 (2006)

[Lucena08] Lucena, M., Santos, E., Silva, C., Alencar, F., Silva, M.J., Castro, J.:Towards a unified metamodel for i*. IEEE Int. Conf. On Research Challenges in Information Science, RCIS 2008. 237 - 246 (2008)

[Lutz93] R.R.Lutz, "Analyzing Software Requirements Errors in Safety-Critical, Embeded Systems", Proceedings RE'93 - First International Symposium on Requirements Engineering, San Diego, IEEE, 1993, 126-133

[Maiden04] Maiden, N.A.M., Jones, S., Manning, S., Greenwood, J., Renou, L.: ModelDriven Requirements Engineering: Synchronising Models in an Air Traffic Management Case Study. CAiSE 2004. LNCS vol. 3084. 368-383 Springer, 2004

[McConnel193] S. McConnell, “Code Complete”, Microsoft Press, 1993

[McConnel196] Steve McConnell, "Rapid Development: Taming Wild Software Schedules" Redmond, Wa.: Microsoft Press, 1996. 660 pages.

[Mehta02] N.Mehta, "About Use Case Maps "Department of Systems and Computer Engineering of Carleton University, http://www.usecasemaps.org/aboutucms.shtml, 2002

[Micmac03] From Requirements to UML Models with Use Case Maps (UCMs) (2003). http://micmac.mitel.com

[Monkewich01] Monkewich, O., Sales, I. and Probert, R.L.: OSPF Efficient LSA Refreshment Function in SDL. Tenth SDL Forum (SDL'01), Copenhagen, Denmark. LNCS 2078, Springer, 300-315, June 2001

[Mussbacher01] G. Mussbacher and D. Amyot, " A Collection of Patterns for Use Case Maps". First Latin American Conference on Pattern Languages of Programming (SugarLoafPLoP 2001), Rio de Janeiro, Brazil, 2001

[Mussbacher07] Mussbacher, G., Amyot, D., and Weiss, M.: Formalizing Patterns with the User Requirements Notation. T. Taibi (Ed.), Design Pattern Formalization Techniques, IGI Global, 304-325, March 2007

[Mussbacher07b] Mussbacher, Gunter. "Evolving Use Case Maps as a Scenario and Workflow Description Language." 10th Workshop on Requirements Engineering (WER 2007). Toronto, ON, Canada, 2007 
[Mussbacher09] Gunter Mussbacher, Sepideh Ghanavati, Daniel Amyot, "Modeling and Analysis of URN Goals and Scenarios with jUCMNav," re, pp.383-384, 2009 17th IEEE International Requirements Engineering Conference

[Mylopoulos98a] Mylopoulos, J.: Information Modeling in the Time of the Revolution. Inf. Syst. 23(3-4): 127-155 (1998)

[Mylopoulos98b] E. Yu, J. Mylopoulos, Why goal-oriented requirements engineering, in: E. Dubois, A.L. Opdahl, K. Pohl (Eds.), Proceedings of the Fourth International Workshop on Requirements Engineering: Foundations of Software Quality, Pisa, Italy, Presses Universitaires de Namur, Paris, June 1998, pp. 15-22

[Mylopoulos99] Mylopoulos, J., Chung, L. and Yu, E: From Object-Oriented to GoalOriented Requirements Analysis. In: Communications of the ACM, Vol. 42, No. 1, January 1999

[Mylopoulos04 ] John Mylopoulos and Steve Easterbrook, "Conceptual Modeling", Information Systems Analysis and Design, csc340, 2004

[NIH07] NIH “Business Process Management (BPM) Service Pattern”, 2007

[Nixon00] Nixon, B.A., "Management of Performance Requirements for Information System", IEEE Transactions on SE, 26(12): 1122-1146,2000

[Nuseibeh00] Nuseibeh, B., and Easterbrook, S: Requirements Engineering: A Roadmap.In: A Finkelstein (Ed), The Future of Software Engineering, ICSE 200-, ACM Press, 2000

[OMG01] OMG (2001), UML Profile for Scheduling, Performance and Time. OMG Document ad/2001-06-14, http://www.omg.org/cgi-bin/doc?ad/2001-06-14, June

[OpenOME] OpenOME. University of Toronto. http://www.cs.toronto.edu/km/openome/

[Pavan03] Pavan, P., Maiden, N.A.M., \& Zhu, X.: Towards a Systems Engineering Pattern Language: Applying i* to Model Requirements Architecture Patterns. ICSE STRAW'03: 2nd Int.Ws. From Software Requirements to Architectures. Portland, Oregon, USA. 134-141 (2003)

[Pengfei 07] Pengfei Chen, "Goal-Oriented Business Process Monitoring. An approach based on User Requirement Notation combined with Business Intelligence and Web Services", 2007

[Perini04] Perini, A., Susi, A.: Developing a decision support system for integrated production in agriculture. Environmental Modelling and Software, Elsevier. 19(9) 821 829 (2004) 
[Petit99] Petit, M.:"Formal Requirements Engineering of Manufacturing Systems : A Multi Formalism and Component-Based Approach", PhD, University of Namur, Belgium, 1999

[Petriu02] D. Petriu and M. Woodside, "Software Performance Models from System Scenarios in Use Case Maps. $12^{\text {th }}$ International Conf. on Modeling Tools and Techniques for Computer and Communications System Performance Evaluation, London, U.K., April 2002

[Philbin 1 1] Simon P Philbin, "Management System for Multidisciplinary University Research Institutes", Institute of Shock Physics \& Visiting, IEEE 2011

[Pourshahid08] Pourshahid, A., and Tran, T.: "Toward an Effective Trust Management System for ECommerce: Modeling Trust Components and Processes Using URN. Journal of Business and Technology (JBT), Atlantic Academic Press, 2008

[Pourshahid08] Pourshahid, A., Chen, P., Amyot, D., Forster, A.J., Ghanavati, S., Peyton, L., Weiss, M., "Toward an integrated User Requirements Notation framework and tool For Business Process Management. 3rd Int. MCeTech Conf. on eTechnologies, Montréal, Canada, IEEE Computer Society, 3-15 (2008)

[Pourshahid09] Pourshahid, A., Chen, P., Amyot, D. "Business Process Management with the User Requirements Notation. Electronic Commerce Research, 9(4), Springer, 269-316, 2009

[Pourshahid12]A. Pourshahid, L. Peyton, S. Ghanavati, D. Amyot, P. Chen, and M. Weiss, "Model-Based Validation of Business Processes" Business Science Reference, IGI Global, 2012

[Quan 11]Lu, Quan, Yue Xia, “ A study of Informationalization and Social Security Management Service" 978-1-4244-8694-6/11/2011 IEEE

[Quillian63] Quillian, R. “A notation for representing conceptual information: An application to semantics and mechanical English paraphrasing. SP-1395, System Development Corporation, Santa Monica, 1963

[Quillian68] M. Ross Quillian. "Semantic Memories", In M. M. Minsky, editor, Semantic Information Processing, pages 216-270. Cambridge, MA: MIT Press, 1968

[Rifaut08] Rifaut, R., Dubois, E.: Using Goal-Oriented Requirements Engineering for Improving the Quality of ISO/IEC 15504 based Compliance Assessment Frameworks. IEEE Int. Conf. on Requirements Eng. RE 2008: 33-42 (2008)

[Rolland98] C. Rolland, G. Grosz, "Experience with goal scenario coupling in requirements engineering" in: Proceedings of the IEEE International Symposium on Requirements, Engineering, 1998, Limerick, Ireland, June 1999 
[Ross77a] D.T.Ross and K.E.Schoman, "Structured Analysis for Requirements Definition", IEEE Transactions on SoftwareEngineering, Vol.3, No.1, 1977, 6-15

[Ross77b]Douglas Ross, "Structured Analysis and Design Technique (SADT)" 1977 [Roy07] Jean-François Roy, "Requirement Engineering with URN: Integrating Goals and Scenarios", Ottawa-Carleton Institute for Computer Science, 2007

[Rowe197] Ramos Rowel and Kurts Alfeche,“ Requirements Engineering A good practice guide" John Wiley and Sons, 1997

[Ryan09] Ryan K. L. Ko, Stephen S. G. Lee, Eng Wah Lee, Business Process Management (BPM) Standards: A Survey. In: Business Process Management Journal, Emerald Group Publishing Limited. Volume 15 Issue 5. ISSN 1463-7154 2009

[Sabetzadeh06] Sabetzadeh, M., Easterbrook, S.: View merging in the presence of incompleteness and inconsistency. Requirements Engineering, 11(3) 174-193. Springer (2006)

[Sage01] Andrew P. sage, "Conflict and risk in systems management as complex adaptive systems issues", Department of Systems Engineering and Operations 0-7803 7087-2/ 2001 IEEE

[Sakkina03] Sakkina Banu Sikandar-gani, "User Requirement Notation (URN)", 2003

[Saleh02] Saleh, Kassem;"Extending Lyee Methodology for Capturing Non-Functional Software Requirements" Project Proposal, October 2002

[Saleh04] K. Saleh and A. Al-Zarouni, "Capturing Non-Functional Software Requirements using the User Requirements Notation", The 2004 International Research Conference on Innovations in Information Technology, pag. 222 to 230

[Sales01] Sales, I., “ A Bridging Methodology for Internet Protocols Standards Development. M.Sc. thesis, SITE, Univ. of Ottawa, Canada, August 2001

[Santader02] Victor F.A. Santander, Jaelson Castro, "Deriving Use Cases from Organizational Modeling," re, pp.32, 10th Anniversary Joint IEEE International Requirements Engineering Conference (RE'02), 2002

[Sawyer01] Pete Sawyer and Gerald Kotonya, "Software Requirements", 2001

[Scratchley99] Scratchley, W.C. and Woodside, C.M., "Evaluating Concurrency Options in Software Specifications", Seventh International Symposium on Modelling,Analysis and Simulation, College Park Maryland, USA, 1999

[Sladic11] G. Sladić, M. Vidaković and Z. Konjović, “Agent Based System for Network Availability and Vulnerability Monitoring", IEEE 9th International Symposium on 
Intelligent Systems and Informatics, September 8-10, 2011, Subotica, Serbia

[Sommerville97] Sommerville, I., and P. Sawyer, "Requirements engineering: A Good Practice Guide", John Wiley and Sons, 1997

[Standish95] the Standish Group, “Software Chaos", http://www.standishgroup.com

[Syracuse911] Syracuse Post Standard, "Speakers Give Sound Advice", March 28, 1911

[Szolovits94] Szolovits, P., Doyle, J., Long, W.J. "Guardian Angel:Patient-Centered Health Information Systems” Technical Report MIT/LCS/TR-604, 1994

http://www.ga.org/ga/manifesto/GAtr.html

[Ulrik 10] Ulrik Franke, Pontus Johnson, Johan König, Liv Marcks von Würtemberg: "Availability of enterprise IT systems - an expert-based Bayesian model" Proc. Fourth International Workshop on Software Quality and Maintainability (WSQM 2010), Madrid

[URN10] User Requirements Notation, UCM User Group 2010, http://www.UseCaseMaps.org

[Vera07] Vera, A. \& Kuntz, L.," Process-based organization design and hospital efficiency. Health Care Management Review", 32(1): 55-65, 2007

[Weiss05] Weiss, M. and Amyot, D.: Business Process Modeling with URN. In: International Journal of E-Business Research, 1(3), 63-90, July-September 2005

[Wiegers03] Wiegers, Karl E, "Software Requirements, Second Edition", Microsoft Press, 2003, ISBN 0-7356-1879-8

[Wieringa96] Wieringa, R. J. “ Requirements Engineering: Frameworks for Understanding”, Wiley, 1996

[Young01] Young, Ralph R., "Effective Requirements Practices", Addison-Wesley, 2001, ISBN 978-0-201-70912-4

[Yu01a] Yu, Eric. Agent Orientation as a Modelling Paradigm, Wirtschaftsinformatik. 43(2), pp.123-132, April 2001

[Yu01b] Yu, E.: Agent-Oriented Modelling: Software Versus the World. In: AOSE 2001. LNCS vol. 2222. 206-225. Springer (2001)

[Yu01c] Yu, E.: Agent Orientation as a Modelling Paradigm, In: Wirtschaftsinformatik, 43(2), April 2001, pp. 123-132

[Yu97] Yu, E.: Why Agent-Oriented Requirements Engineering. In: Proc. 3rd Int. Workshop on Requirements Engineering: Foundations of Software Quality (REFSQ'97), 
Barcelona, Spain, June 1997, pp. 171-183

[Yu02] E. Yu, L.M. Cysneiros, "Designing for Privacy and Other Competing Requirements", 2002, In Proceedings of the 2nd Symposium on Requirements Engineering for Information Security (SREIS-02)

[Yu03] E.Yu , L.Cysneiros, Designing for Privacy in a Multi-Agent World, 2003

[Yu04a] Yu, E.S.K., Mylopoulos, J.: From E-R to A-R: Modelling Strategic Actor Relationships for Business Process Reengineering. In: P. Loucopoulos (Ed.), ER 1994. Manchester, U.K., LNCS vol. 881, 548-565. Springer-Verlag (2004)

[Yu04b] Yu, Y., S.P.Leite, J.C., and Mylopoulos, J.: From Goals to Aspects: Discovering Aspects from Requirements Goal Models: In: Proceeding of the 12th IEEE International Requirements Engineering Conference (RE'04), Kyoto, Japan, September 2004

[Yu05] Yu, E.: OpenOME, an open-source requirements engineering tool, 2005. http//www.cs.toronto.edu/km/openome/

[Yu09] Yu, Eric (2009) Social Modeling and i*. In: Borgida, A. T., V. Chaudhri, P. Giorgini, E. S. Yu (eds.): Conceptual Modeling: Foundations and Applications - Essays in Honor of John Mylopoulos. LNCS volume 5600. Springer. ISBN 978-3-642-02462-7

[Yu97] Yu, E.: Towards Modeling and Reasoning Support for Early-Phase Requirements Engineering, In: Proceedings of the 3rd IEEE Int. Symp. On Requirements Engineering (RE'97), Washington D.C., USA, 226-235, January 1997

[Yu98] Yu, E. and Mylopoulos, J.: Why Goal-Oriented Requirements Engineering. In: Proceedings of the 4th International Workshop on Requirements Engineering Foundations of Software Quality, Pisa, Italy, 15-22, 1998

[Zachman87] Zachman, J.A.: A Framework for Information Systems Architecture. IBM Systems Journal, 26(3) 1987

[Zave97] Zave,P.: Classification of Research Efforts in Requirements Engineering. In: ACM Computing Survey, 29(4), 315-321, 1997

[Zheng 06] K. Zheng, K. Hoffman: "A Service-Oriented Architecture in a Multi-Agency Environment: A Case Study in Enterprise Dynamics", 10th IEEE International Enterprise Distributed Object Computing Conference Workshops (EDOCW'06)

[Zhimei00] Zhimei Y., "CNAP Specification and Validation: A Design Methodology Using LOTOS and UCM", 2000

[Zhiming02] Zhiming Cai and Eric Yu, "Addressing Performance Requirements Using a Goal and Scenario-Oriented Approach”, 2002 


\section{Appendices}

\section{Appendix A - Administrative information propagation}

\section{Case working system}

For this option we are working with three strategies indicated in (Figure A 1 and Figure A2). We proceed with the evaluation process as presented before: we execute each strategy and we verify the results obtained without and with KPIs.

UCM Scenarios

GRL Evaluation Strategies

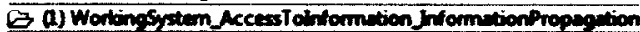

G (2)WPI Workingsystem_Access Tolnformution_Information Propuge

Figure A 1 : Strategy list information
, GR Evalution Strutegies

Q (1) Workingsyten_AccessTalitomation Jormutionpropagation (11126)

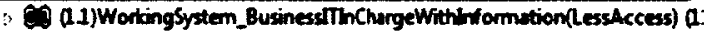

- (1.2)WorkingSytem_Busines:Th ChargeWithlhformationAccess) (16097)

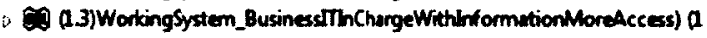

FigureA 2: Strategies summary

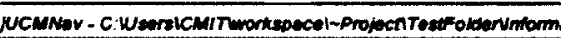

\begin{tabular}{l|l|l|l}
$\begin{array}{l}\text { ResoveRequ } \\
\text { ests }\end{array}$ & -26 & 28 & 86 \\
\hline $\begin{array}{l}\text { ReportData } \\
\text { Intomation }\end{array}$ & 30 & 31 & 54 \\
\hline
\end{tabular}

FigureA 3:Extras from .pdf file (realization of goals)

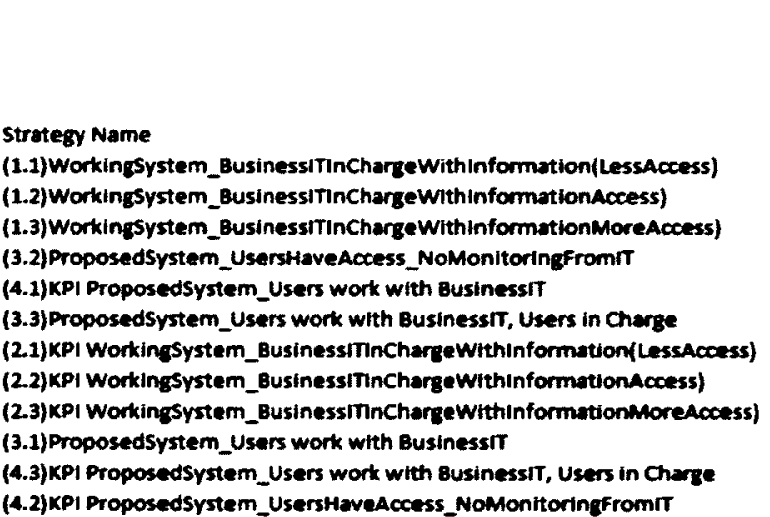

\begin{tabular}{|c|c|c|c|}
\hline $\begin{array}{l}\text { Identify } \\
\text { Problems } \\
\text { Onspecific } \\
\text { component }\end{array}$ & $\begin{array}{c}\text { Identify } \\
\text { Problems } \\
\text { on } \\
\text { Developm } \\
\text { ent }\end{array}$ & $\begin{array}{l}\text { Impose } \\
\text { Corrections } \\
\text { Schedule }\end{array}$ & $\begin{array}{l}\text { Impose } \\
\text { Deployments }\end{array}$ \\
\hline -73" & $-25 *$ & -6811 & $-20 \%$ \\
\hline$-25 *$ & $-12 *$ & $-18 *$ & $25 *$ \\
\hline $35 n$ & $2 *$ & 44" & $76 n$ \\
\hline ts* & $25 *$ & 90* & $85 \%$ \\
\hline $37 \%$ & 6* & $45 \#$ & $76 \%$ \\
\hline 81* & 187 & 90" & 76 \\
\hline$-75 *$ & $-25 *$ & $-68 n$ & $-20=$ \\
\hline$-25 n$ & $-12 *$ & $-18 n$ & 23* \\
\hline $35 n$ & 2* & 44: & $26 \%$ \\
\hline $37 \%$ & 6* & 45* & $76 \%$ \\
\hline 81" & 18* & sow & $76=$ \\
\hline TS* & 25" & son & 85\% \\
\hline
\end{tabular}

FigureA 4:Extras from .csv file (realization of goals) 
TableA 1: Evaluation of goals, Information propagation, working system

\begin{tabular}{|c|c|c|c|c|c|c|}
\hline $\begin{array}{l}\text { Eviluation } \\
\text { Strutegy }\end{array}$ & 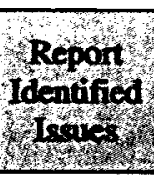 & 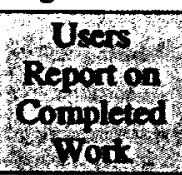 & Wrondod to Cient & 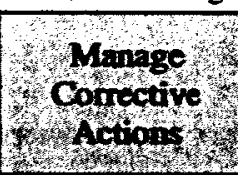 & 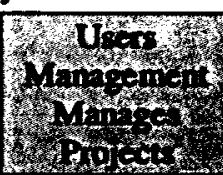 & 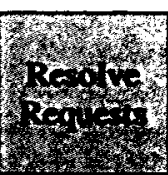 \\
\hline 1.1 & -54 & -25 & 4 & -68 & -45 & -26 \\
\hline 1.2 & -13 & 14 & 17 & -18 & -2 & 28 \\
\hline 1.3 & 38 & 73 & 48 & 44 & 58 & 86 \\
\hline
\end{tabular}

TableA 2: KPI Evaluation of goals, Information propagation, working system

\begin{tabular}{|c|c|c|c|c|c|c|c|}
\hline 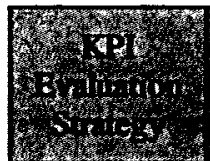 & 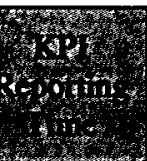 & 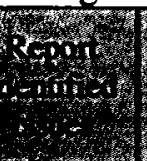 & 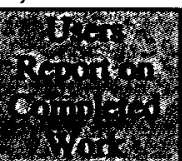 & 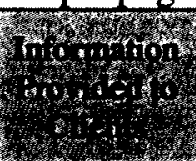 & Why & 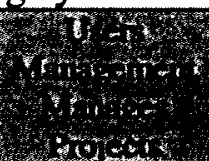 & (5) \\
\hline 2.1 & -10 & -54 & -27 & 4 & -68 & -45 & -26 \\
\hline 2.2 & 3 & -13 & 14 & 17 & -18 & -2 & 28 \\
\hline 2.3 & 25 & 38 & 79 & 48 & 44 & 59 & 86 \\
\hline
\end{tabular}

For the KPI Model we defined KPI Reporting Time, as the time elapsed in between the moment the users have a request to address or an issue to report on and the moment the requests has been acknowledged on the other end. For the best situation, we set the Target value for $37.50 \mathrm{~h}$, this is composed of the reporting time to $\mathrm{UM}$, then to $\mathrm{BD}$, then to $\mathrm{BM}$, back to $\mathrm{BD}$ and back to the users.

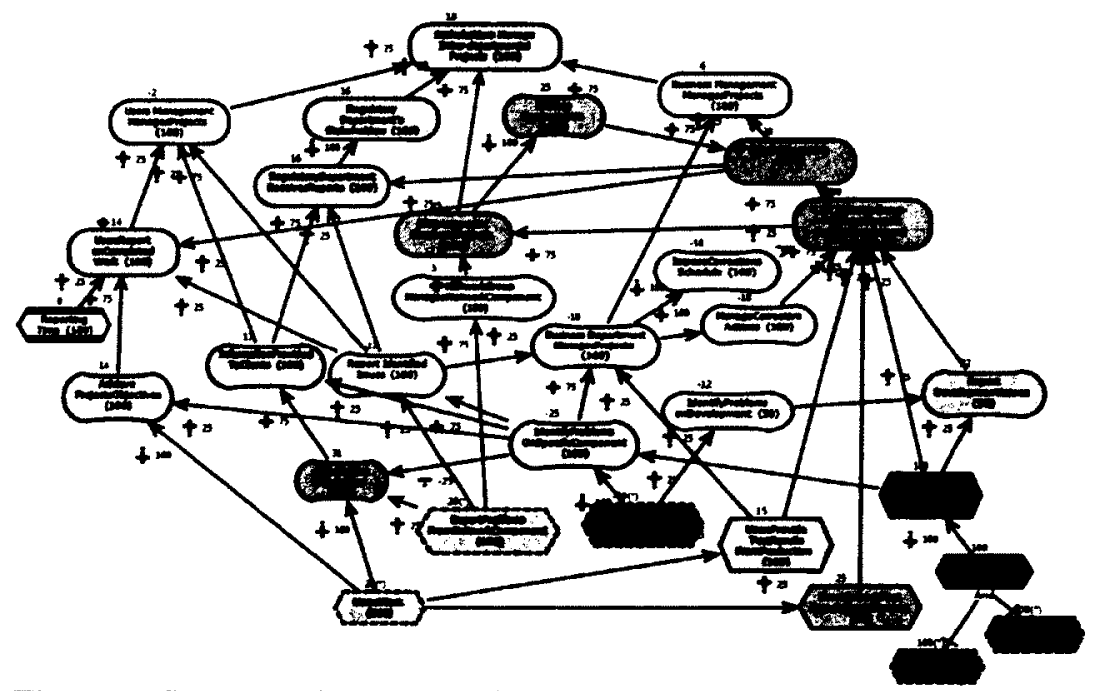

FigureA 5:Evaluation of administrative information propagation (Strategy 1.2) 
For a convenient case, when staff is available to work during regular work hours, excluding mandatory holidays and other vacation times, we considered one working day, $7.50 \mathrm{~h}$ to address the request with anybody from UM; then we considered 2 working days, $15.00 \mathrm{~h}$, to address the request with $\mathrm{BD}$, and 2 working days to have the request addressed by $\mathrm{BD}$ with $\mathrm{BM}$, back to $\mathrm{BD}$, and back to the users. In total we have 5 days or $37.50 \mathrm{~h}$. In many cases $\mathrm{BD}$ will have to follow up with ITD, which could require in average, from 2 working days, or $15.00 \mathrm{~h}$, up to few weeks, depending on the importance and work required by the users request; then we consider other 2 days for the ITD to arrange details with ITM, and to get back to BD.

\section{Case proposed system}

To introduce the model for the proposed systemwith components, strategies (Fig.5.27) (we use three GRL and three KPI strategies), evaluations, we used the same approach as for the working system: present the model execute and gather the evaluation results.

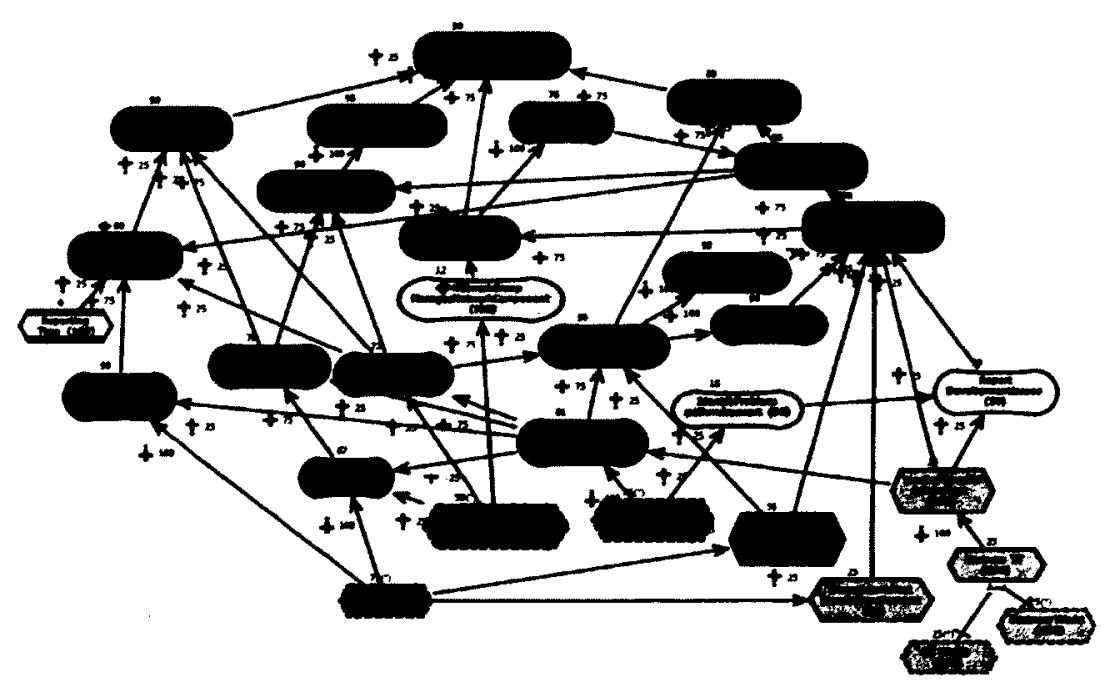

FigureA 6:Evaluation of Administration Information propagation model, Strategy (1.6) 


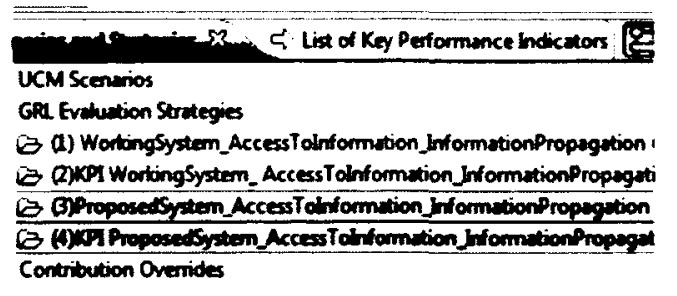

$B$ (3)ProposedSystem_Access Tolnformation_InformationPropagation (1) (1)Proposed System_Users work with Businessit (16619)

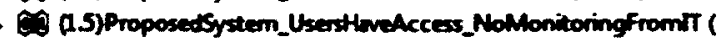
OS (1.6)ProposedSystem_Users wort with Businesst, Users in Char $\Theta$ (4)KPI ProposedSystem_AccessTolnformition_InformationPropaga O (2A)KPI Proposedsystem_Uses work with BusinessT (16599)

3.3 (25)KPI Proposedsystem_UsershoveAccess_NoMonitoring Frorr OS (2.6)KPI ProposedSystem_Users work with BusinessT, Users in C

FigureA 7:Strategies overview

TableA 3: Evaluation of component elemenst (proposed system)

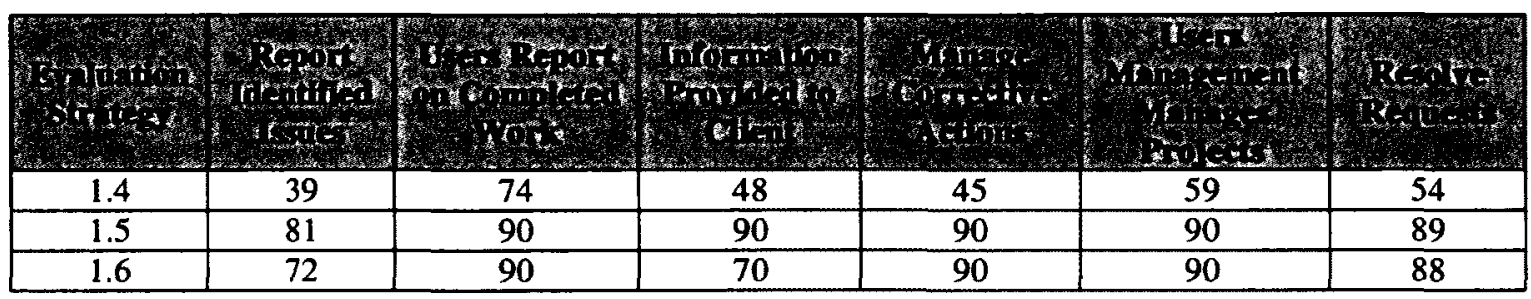

TableA 4: KPI Evaluation of component elements (proposed system)

\begin{tabular}{|c|c|c|c|c|}
\hline Shinging & Penthe & Hopot & (0) & pron \\
\hline 2.4 & 40 & 39 & 84 & 48 \\
\hline 2.5 & 96 & 81 & 90 & 90 \\
\hline 2.6 & 84 & 72 & 90 & 70 \\
\hline
\end{tabular}

TableA 5: KPI Evaluation of component elements (proposed system)

\begin{tabular}{|c|c|c|c|}
\hline 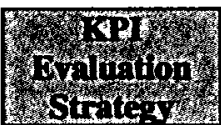 & Whombs & W & 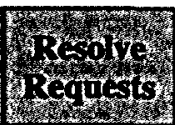 \\
\hline 2.4 & 45 & 62 & 86 \\
\hline 2.5 & 90 & 90 & 88 \\
\hline 2.6 & 90 & 90 & 86 \\
\hline
\end{tabular}




\section{Appendix B - First test then deploy}

\section{Case working system}

This process is also based on the Users work on the system; they can connect and work on two system components: the 'Specific Component' (SC), the extranet also known as production, the development environment of the SC, and the 'Network Component' (NC) or the intranet. These components are running on different network segments that are shared by services and processes running on the network, updates and maintenance work for the majority of the SO across the enterprise, users activities and connections with different databases and clients. The Users have very limited knowledge on the way network service and processes are handled; it is understood though that the operational business and systems of each sub-organizations are administered "to improve working efficiency and service quality", being based on "standardized procedure(s) and a clear operating process(es) for all types of business in order to treat all" sub-organizations "fairly" [Quan11]. If issues are identified by the Users, they have to be documented and reported according to specific rules: the issues found on development will be addressed directly by email with the ITDSC; the issues identified on production will be addressed through CARs with BD, and they will further work with ITDSCl; while the issues identified on the intranet have to be reported through open call requests with the ITDNG. The issues identified on the Users side could be of different priorities and impacts on the Users work, which might not always align with the priorities of the other SO, including $\mathrm{BD}$ and ITD. It is important to have them reported and addressed as soon as possible, to minimize their impact on the Users work. Since the Users document and report such issues, it is important to close the correction loop with BD and ITD, as soon as solutions 
have been found, documented, resolved, tested and deployed, including updating of requirements. In most of the cases, during the resolution of an issue and after the release, the Users were required to participate into testing processes on the SC.

There are three options to work on the system which are dependent on the available budget, staffing, and operational projects happening already. For example, the Users need to have an enhancement implemented on their system, which is not required by the LD, yet is important as a clients request; such situation might not place as a high request in the BD and ITD ranking system, and therefore it could be deferred to a later projects of to the activities planned for the next fiscal year. In such a case, if the Users receive approval from own management and stakeholders, and also support from BD and ITD, the Users might go ahead with a contract and hire a contractor to work out the changes.

In other situations, and only if budget and staff from the other departments is available, ITD and BD could look into such request; we also believe that the Users could work on the requests if they would have some of the technical and business capabilities and responsibilities the other SO have. We included this option in the model and we hope to demonstrate that the evaluation results and satisfaction levels of the goals will be. beneficial for the realization of the Users goals. We have designed the strategies according to this three options for handling a request: by BD and ITD, and potential the a contractor, as it happens in the working system, and by Users, as we aim to present for the proposed system. To be able to administer better the different strategies for the working system version and for the proposed system for each of the three cases, for this particular option only, we modeled the proposed option in the same model file as the working system; therefore we will see the strategies for the proposed system also listed in 
the GRL model, below the strategies corresponding to the working system, (as seen in

Fig.B1, Fig.B2, Fig.B3, Fig.B4) even though the first case, for example, they have a

unique ID and the strategies inside are differentiated by unique identities.

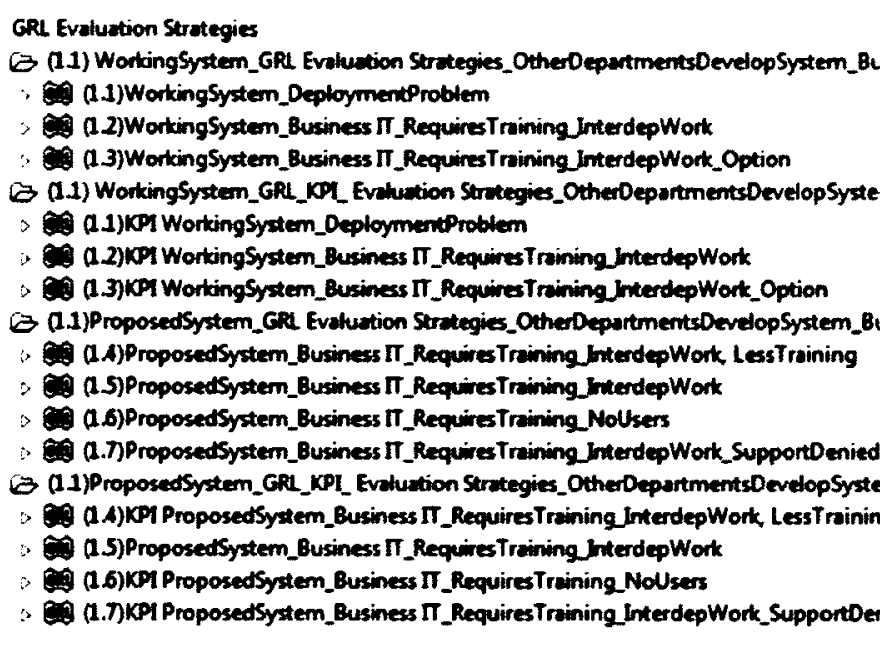

FigureB 1:Extras from strategy list for test and

\section{Deploy}

GRL Evaluation Strategies

$B$ (1.1) WorkingSytem_GRL Evaluation Stritegies_OtherDeportmentsDevel o (11)WorkingSystem_DeploymentProblem

O. (1.2)WortingSystem_Business II_Requires Training JinterdepWork

O] (1.3)WorkingSystem_Business II_RequiresTrining InterdepWork_Opt

6) (11) WorkingSystem_GRL_KPI_Evalution Stritegies_OtherDepartments

Of (1.1)KPI WorkingSystem_DeploymentProblem

OS (12)KPI WorkingSystem_Business II_RequiresTruining JnterdepWork

OS (1.3)KPI WorkingSystem_Business IT_RequiresTrining_interdepWork.

(1.2) WorkingSystem_GRL Evaluation Strategies_ContractorDevelopsSys

O. (2.2)WorkingSystem_Contractor_Develops(Sutisfied, Weedtystisfiex 3 (1.2) WortingSystem_GRL_KPI_Eveluntion Strategies_ContractorDevelos - CO (2.1)KPI WorkingSystem_Contractor_Develops(Sintisfied, WedtySiti: D. (2.2)KPI WorkingSystem_Contractor_Develops(Sutisfied, WeeldySiti:

FigureB 2: Summary of strategies for case Other Departments and case Contractor develops

\begin{abstract}
- (13) WortingSystem_GRL Evaluntion Stritegies_PurchaseProduct OS (3.1) WorkingSystem_PurchaseProduct

E] (3.2) WorkingSystem_PurchsseProduct_NolvserContribution - (1.3) WorkingSystem_GRL_KPI_Evaluation Strategie_PurcheseProduct O. (B.1) WorkingSystem_KPiPurchaseProduct Fi (3.2) WorkingSystem_KPIPurchaseP roduct_NoUserContribution
\end{abstract}

FigureB 3: Summary of strategies for case Purchase Product

\author{
(1.4) WortingSyatem_GRL Evaluation Strategies_UserDevdopsSystem \\ Q.9 (4.1)WorkingSystem_Usersoevelop_OtherDept_Troining \\ OS (4.3)WorkingSystem_Usersoevelop_NoTraining \\ (1.A)GRL_KPIEvuluation Strategies_UserDevelopsSystem \\ QI (11)GRL_XPTWorkingSystem_Usersoevelop_OthedDept_Trining \\ OS (1.2)GRL_KPWorkingSystem_Users Develop_OaherOept_NoContractor_Training
}

FigureB 4: Strategy list for case Users develop

We will analyze the model and the strategies created for each case, looking at the realization of the goals and KPI results. To introduce the model for each of the following cases, we will like to use the same presentation style as the one applied to the previous use cases. In order to normalize the presentation of the cases process, we will have an introductory paragraph only once, here, to avoid to duplicate information in each case. Therefore we will design the model depicting the main actors and their significant goals 
with impact on the users activity and in relation with the Users responsibilities; we will propose different strategies for reaching the objectives, which will be then evaluated to allow us to investigate the evaluation results, satisfaction levels of the goals and performant estimates. In the KPI Model, we used the same two types of KPIs for all cases: Cost to correct, Turnaround Time, defined as:

- Cost to correct, test and deploy fixes - is the financial commitment the Users owe to BD and ITD for manage corrective actions requests, enhancements and any other fixes. Each year the users have to estimate the budget for system maintenance which will also refer to corrections and system additions. Therefore the target value of the KPI will be set on the maintenance budget, leaving some amount aside to cover the threshold value.

- Turnaround Time - represents the time elapsed in between addressing a request to $\mathrm{BD}$ or ITD by the Users, and receiving back the response/resolution to it. The resolution time in our case study it includes other time intervals aside the time elapsed since the reporting of the problem and the disposing; the extra time intervals are composed of: the time the request is pending to be acknowledged by the $\mathrm{BD}$, giving that all the requests received from all the sub-organizations are being placed in a queue; the time the request is pending the be addressed by the $\mathrm{BD}$, and receive a request number and the ranking from $\mathrm{BD}$, even though the request already has a ranking priority expressed by the User; the time the request needs to be analyzed by the $\mathrm{BD}$, documented and to receive a resolution, which depends by own $\mathrm{BD}$ operational goals and projects set for the current year. If the request received high priority ranking from $\mathrm{BD}$ and has been included in the corrections estimated for the year, than BD will inform the Users and will further address the request with ITD SC; the time is needed to have the request acknowledged by the 
ITD, given that IT provides services for all the sub-organizations from the organization parent; the time needs to address the request, the time spent to recreate the request in the development environment, the time spent to document the issue, the time required to find different solutions, the time needed for programming, the time required to test the solutions, the time needed to document the request and the time required to estimate the cost of the solutions. To this time interval we continue to add the time is needed to provide ITD's feed-back to the $\mathrm{BD}$; the time required by the $\mathrm{BD}$ to further provide the feedback to the Users; the time required from the Users by the ITD to analyze the solutions proposed and to test the solutions on the Users production system; the time the users must document the results of the testing and send them back to IT; the time required by ITD to compare the test results; the time required by IT to commit all programming changes in the new file to be realeased; the time needed to coordinate the deployment with the $\mathrm{BD}$, ITD various groups from the front end and back end administrations; the time needed to deploy the changes; the time required from the Users by ITD to test again the changes on production and confirm that the problem has been resolved; the time required by $\mathrm{BD}$ to close the case opened for this request, and confirm it with the users; the time required by $\mathrm{BD}$ to update the Users system documentation

\section{Case 1) Other Departments (ITD and BD) provide support in maintenance}

We will present each case by introducing the strategies, next will execute the model and collect the evaluation results. We will also execute the model with the KPIs enable, to look at their contribution / impact on the realization of the goals. Here we have listed the strategies corresponding to this case; they are related to the working system (from 1.1 to 1.3 in Figure B5) and from (1.4 to 1.7 in Figure B6) to the proposed system; and the rest 
are the strategies corresponding to KPI for both types. Due to space limitations in our results tables we will present the results for the goals and actors that contribute to the realization of Users goals.

3 (1.1) WorkingSystem_GRL Evaluation Strategies_OtherDepartments.0ev a a.1)WorkingSystem_DeploymentProblem (4971)

O3 (1.2)WorkingSystem_Business T_RequiresTraining_hterdepWork (3 C3 (1.3)WorkingSystem_Business $\pi_{-}$RequiresT raining_InterdepWork_C 3 (1.1) WorkingSystem_GRL_KPI_Evaluation Strategies_OtherOepartment - C2 (11)KPI WorkingSystem_DeploymentProblem (19006)

- 2) (1.2)KPI WorkingSystem_Business II_RequiresTraining_InterdepWo

- 03 (1.3)KPI WorkingSystem_Business II_RequiresTraining_InterdepWor

FigureB 5: Details on Strategies for OtherDepartments

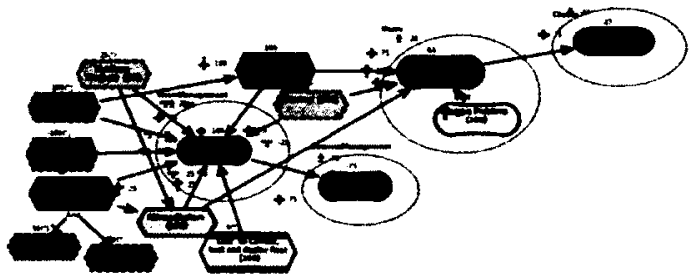

FigureB 7: Other Departments, Evaluation of Strategy (1.1)

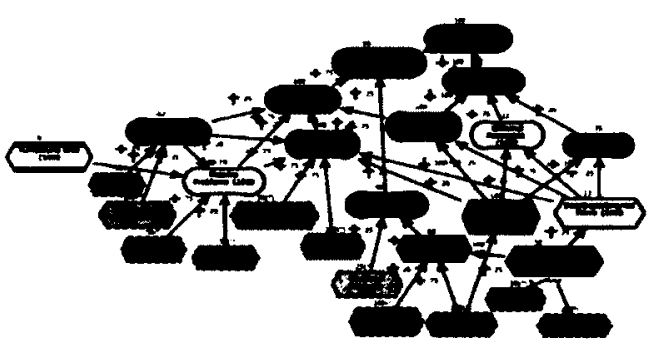

FigureB 9: Other Departments, detailed graph, Evaluation Strategy (1.1)

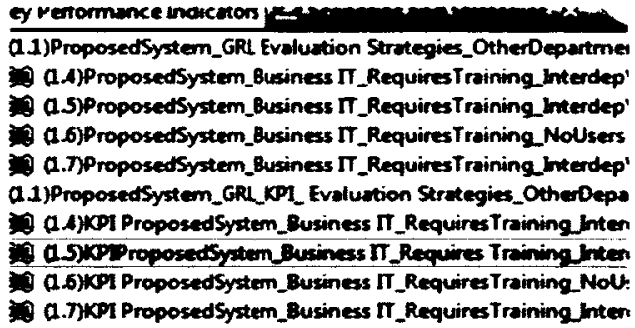

FigureB 6: Details KPI Strategies for Other Departments

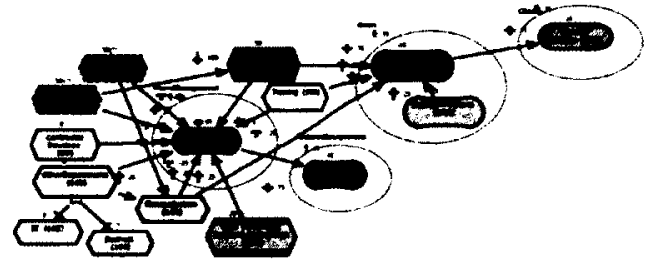

FigureB 8: Other Departments, Evaluation of Strategy (1.6)

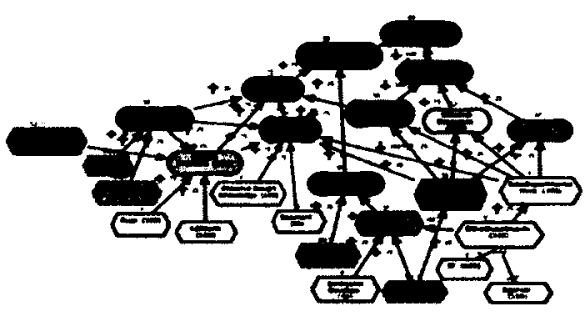

FigureB 10: Other Departments, detailed graph, Evaluation Strategy (1.6)

The results of the evaluation of the model per strategies are presented in the tables below, as follows: (TableB1, TableB2) 
TableB 1: Evaluation of goals per actors (UM , Users, Clients, working/proposed)

\begin{tabular}{|c|c|c|c|c|}
\hline \multirow{2}{*}{$\begin{array}{l}\text { Erraluation } \\
\text { Strategy }\end{array}$} & 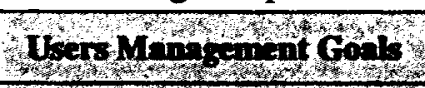 & \multicolumn{3}{|c|}{ 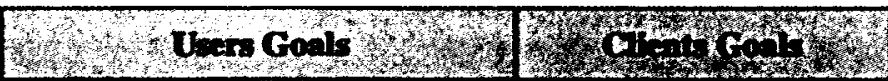 } \\
\hline & 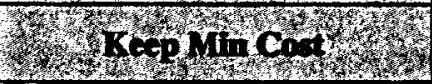 & Probans & Conitionts to & W \\
\hline \multicolumn{5}{|c|}{ Category A: working system } \\
\hline 1.1 & -100 & -46 & -63 & -47 \\
\hline 1.2 & 35 & 10 & 7 & 5 \\
\hline 1.3 & -4 & -46 & 0 & $\mathbf{0}$ \\
\hline \multicolumn{5}{|c|}{ Category B: proposed system } \\
\hline 1.4 & 37 & 59 & 63 & 47 \\
\hline 1.5 & -42 & 29 & 25 & 18 \\
\hline 1.6 & 59 & 12 & 43 & 32 \\
\hline 1.7 & 90 & $\mathbf{0}$ & 31 & 23 \\
\hline
\end{tabular}

TableB 2: KPI Evaluation of goals per actors (UM , Users, Clients, working/proposed)

\begin{tabular}{|c|c|c|c|c|}
\hline and & $\frac{67}{67}$ & os & 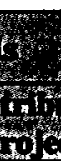 & \\
\hline Categor & & & & \\
\hline 1.1 & -100 & 12 & -62 & -46 \\
\hline 1.2 & 35 & -58 & 4 & 3 \\
\hline 1.3 & -4 & -57 & -3 & -2 \\
\hline Categor & & & & \\
\hline 1.4 & 37 & 81 & 69 & 51 \\
\hline 1.5 & -42 & 43 & 28 & 21 \\
\hline 1.6 & 59 & 25 & 46 & 34 \\
\hline 1.7 & 90 & 7 & 32 & 24 \\
\hline
\end{tabular}

TableB 3:KPI Evaluations of goals, KPI Turnaround time, Other Departments, working/proposed

\begin{tabular}{|c|c|c|c|c|c|}
\hline Shing & Whing & 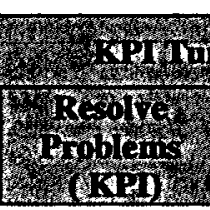 & 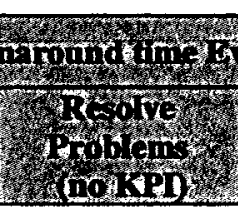 & 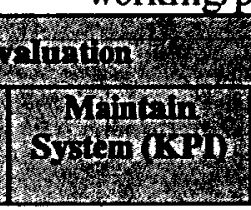 & 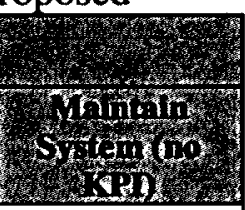 \\
\hline Category A: v & orking system & & & & \\
\hline 1.1 & 10 & 12 & 10 & -100 & -100 \\
\hline 1.2 & -48 & -58 & -46 & -61 & -52 \\
\hline 1.3 & -46 & -57 & -46 & -67 & -59 \\
\hline Category $B: p$ & oposed system & & & & \\
\hline 1.4 & 91 & 81 & 59 & 90 & 90 \\
\hline 1.5 & 58 & 43 & 29 & 56 & 42 \\
\hline 1.6 & 52 & 25 & 12 & 77 & 66 \\
\hline 1.7 & 29 & 7 & $\overline{0}$ & 46 & 40 \\
\hline
\end{tabular}


TableB 4:KPI Evaluations of goals, KPI Turnaround time, Cost to correct,test working/proposed

\begin{tabular}{|c|c|c|c|c|c|}
\hline 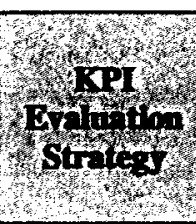 & 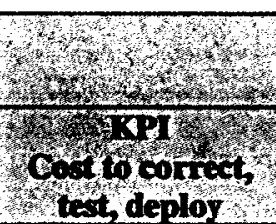 & 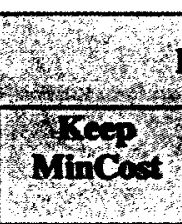 & 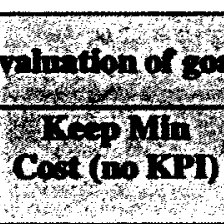 & 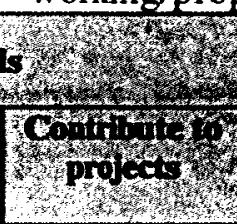 & 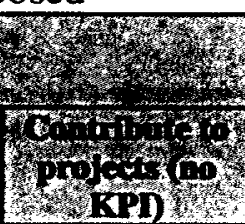 \\
\hline \multicolumn{6}{|c|}{ Working system } \\
\hline 1.1 & -100 & -100 & -100 & -62 & -63 \\
\hline 1.2 & 30 & 35 & 35 & 4 & 7 \\
\hline 1.3 & -9 & -4 & -4 & -3 & $\overline{0}$ \\
\hline \multicolumn{6}{|c|}{ Proposed system } \\
\hline 1.4 & 37 & 37 & 37 & 69 & 63 \\
\hline 1.5 & 0 & -42 & -42 & 28 & 25 \\
\hline 1.6 & 25 & 59 & 59 & 46 & 43 \\
\hline 1.7 & 44 & 90 & 90 & 32 & 31 \\
\hline
\end{tabular}

The evaluation values and contribution of the KPIs are presented in the tables above and images from below, grouped by strategy type. For small size tables, (see Table B5 and Table B6 per below) for convenience, we have displayed aside, both evaluation results, obtained without and with KPI, the results obtained without KPI.

TableB 5: Evaluation of Actors

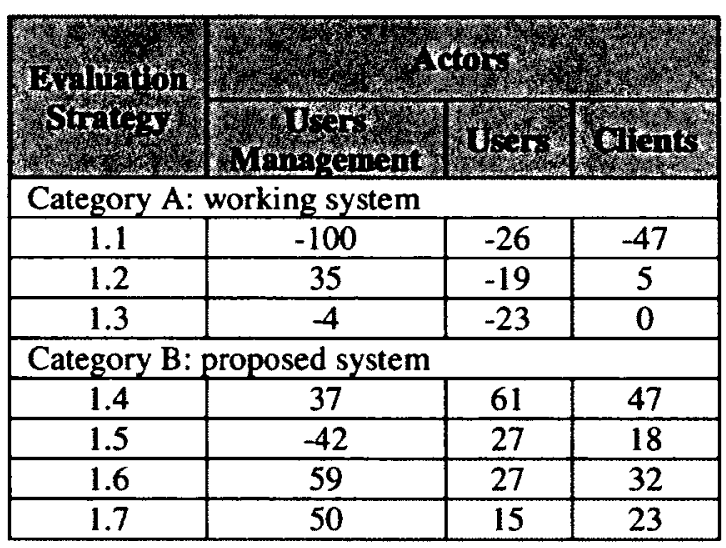

TableB 6:KPI Evaluation of Actors

\begin{tabular}{|c|c|c|c|}
\hline 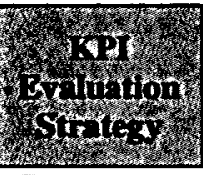 & 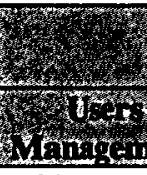 & 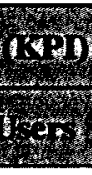 & 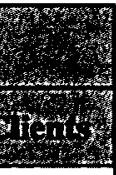 \\
\hline Category A: & orking sys & & \\
\hline 1.1 & -100 & -25 & -46 \\
\hline 1.2 & 35 & -27 & 3 \\
\hline 1.3 & -4 & -30 & -2 \\
\hline Category B: & roposed sy: & & \\
\hline 1.4 & 37 & 75 & 51 \\
\hline 1.5 & -42 & 35 & 21 \\
\hline 1.6 & 59 & 35 & 34 \\
\hline 1.7 & 90 & 19 & 24 \\
\hline
\end{tabular}

\section{Case 2c) Contractor develops}

In Figure B11 and Figure B12 we are presenting the strategies developed for this case, for both system types (working and proposed) and both types of strategies (GRL, with no 
KPI, and the KPI ones). The strategies are related relate to the working system (from 2.1 to 1.2 in Figure B 11) and from (2.3 to 2.5 in Figure B 12) to the proposed system; the rest are the strategies corresponding to KPI for both system types, working and proposed.

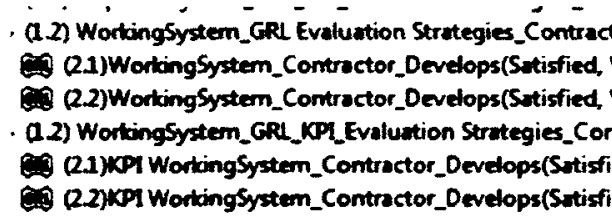

FigureB 11: Strategies List,

Contractor develops, working system

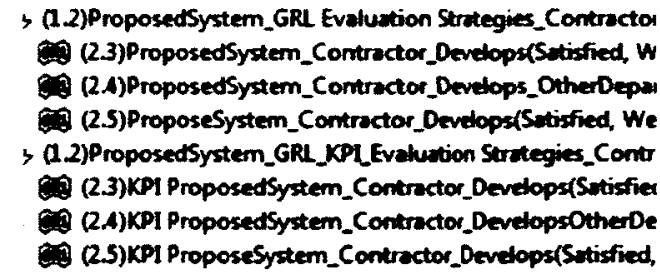

FigureB 12; Strategies List, Contractor

Develops proposed system

We will execute the model for each strategy and acknowledge the evaluation results generated in accordance to the characteristics of the strategies.

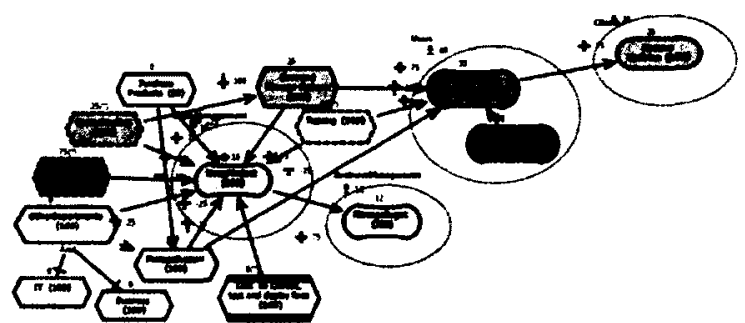

FigureB 13: Evaluation Contractor Develops, Strategy (2.1)

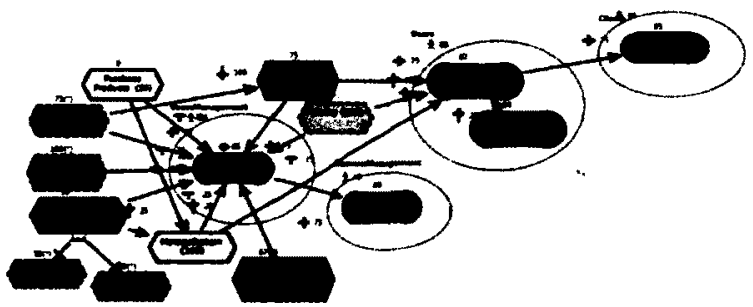

FigureB 14: Evaluation Contractor Develops, Strategy (2.4)

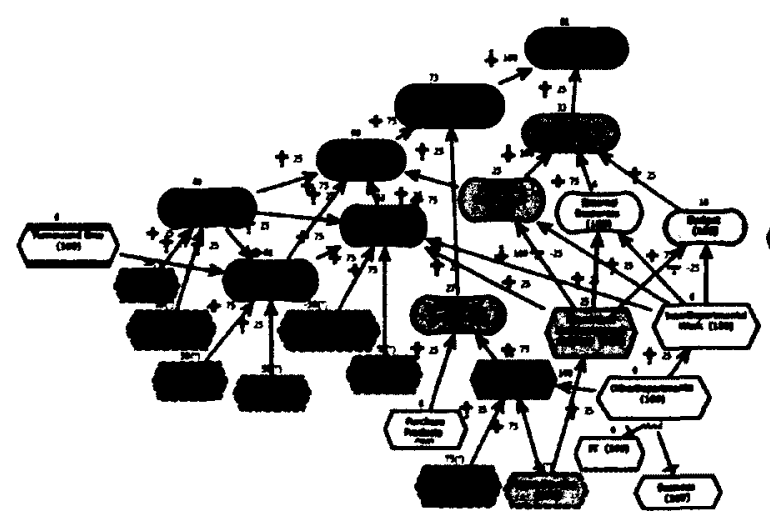

FigureB 15:Evaluation, Contractor Develops, Strategy (2.1)

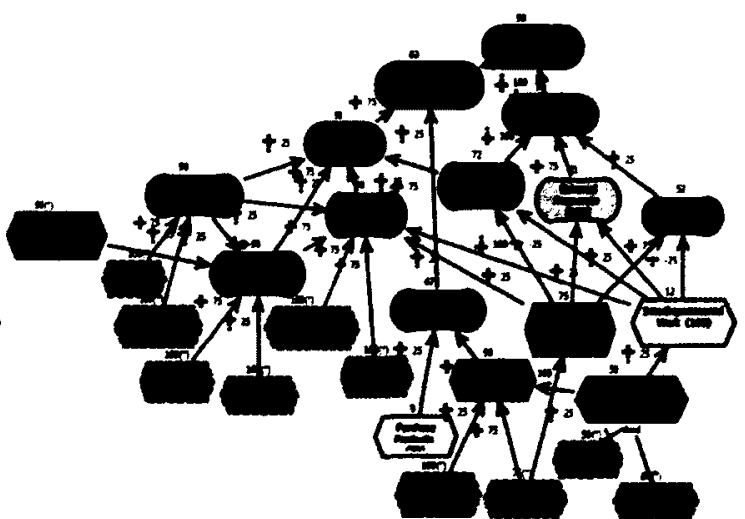

FigureB 16: Execution of model for Strategy (2.4) 
Strategy Name

(1.3)WorkingSystem_Business $\pi_{-}$RequiresTraining_interdepWork_Option

(1.2)WorkingSystem_Business $\pi$ _RequiresTraining_Interdepwork

(2.1)Work'n_system_Contrector_Demalope/Setisfied, Wenidysetisfied so for Traln

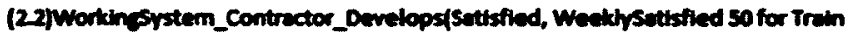

(1.1)WorkingSystem_DeploymentProblem

(3.1) Workin System_DurchaseProduct

(21)KP) WorkingSystem_Contractor_Develops(Satisfied, Weeklysatisfied 50 for $T 1$

(2.2)KP! WorkingSystem_Contractor_Develops(Sotisfied, Weeklysatisfied 50 for $T$

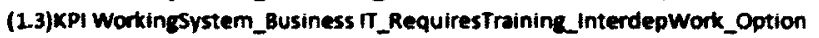

(1.2)KPI WorkingSystem_Business IT_Requirestraining_interdepWork

(1.1)KPi WorkingSystem_DeploymentProblem

$\begin{array}{cc}\begin{array}{c}\text { Users } \\ \text { Management }\end{array} & \begin{array}{c}\text { Business } \\ \text { Management }\end{array} \\ \text { (A) } & \text { (A) } \\ 4 & -3 \\ 35 & 26 \\ 16 & 12 \\ -100 & -75 \\ -100 & -75 \\ 39 & 29 \\ 16 & 12 \\ -100 & -75 \\ -4 & -3 \\ 35 & 26 \\ -100 & -75\end{array}$

Clients Develop Purchase Users

(A) System Products Develop

\begin{tabular}{|c|c|c|c|}
\hline 0 & 18* & -100 & on \\
\hline 5 & on & -100 & 10 \\
\hline 25 & 3 & $\infty$ & 23 \\
\hline-23 & -4 & -100 & -100 \\
\hline-47 & $-88 *$ & 25 & -100 \\
\hline-8 & $-43 *$ & 100 & 10 \\
\hline 29 & $36 *$ & O* & 25 \\
\hline-25 & $-45 *$ & -100 & -100 \\
\hline-2 & 18* & -100 & o* \\
\hline 3 & 0 & -100 & 10 \\
\hline-46 & $-88 *$ & 25 & -100 \\
\hline
\end{tabular}

FigureB 17: Extras from Contractor Develops .csv report

TableB 7: Evaluation of goals per Actors (UM, Users, Clients), Contractor Develops, working/proposed

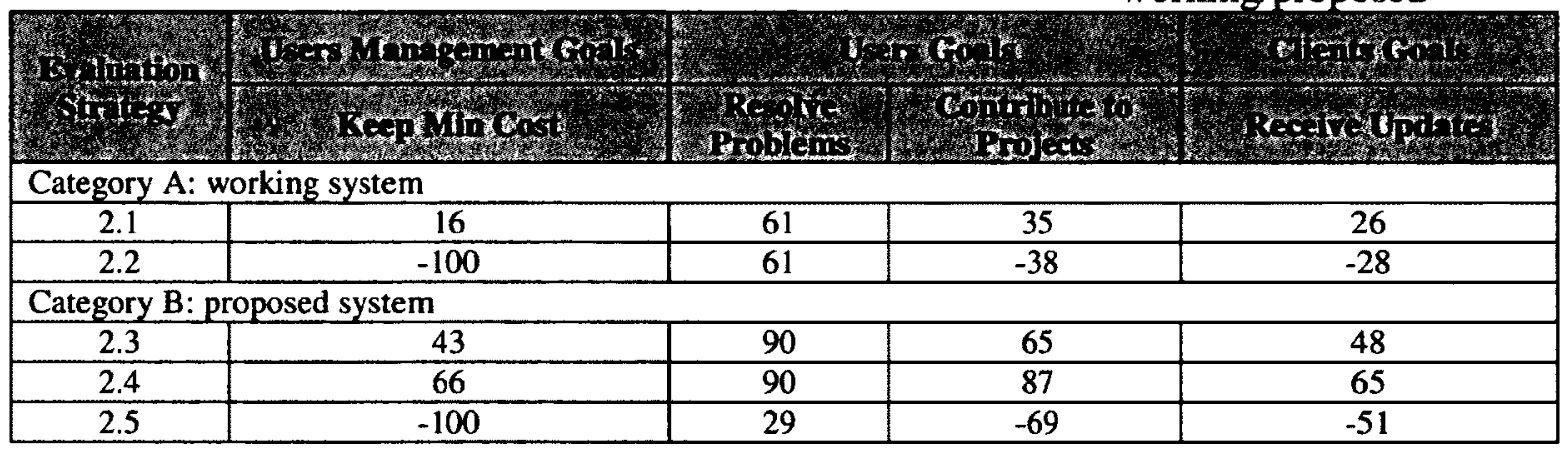

The evaluation values and contribution of the KPIs are presented in the tables listed

below, with the results grouped by strategy type.

TableB 8: KPI Evaluation of goals per Actors (UM, Users, Clients), Contractor

Develops, working/proposed

\begin{tabular}{|c|c|c|c|c|}
\hline 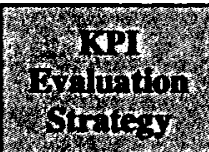 & 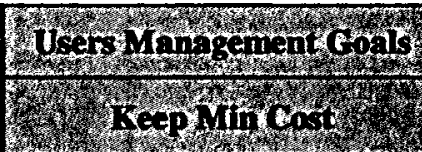 & 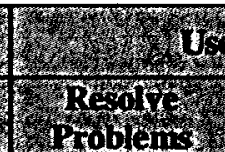 & 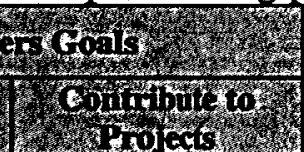 & 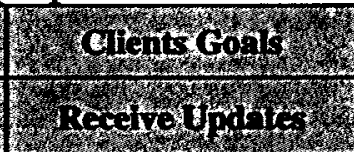 \\
\hline \multicolumn{5}{|c|}{ Category A : Working system } \\
\hline 2.1 & 16 & 76 & 39 & 29 \\
\hline 2.2 & -100 & 76 & -34 & -25 \\
\hline \multicolumn{5}{|c|}{ Proposed system } \\
\hline 2.3 & 43 & 90 & 65 & 48 \\
\hline 2.4 & 66 & 90 & 87 & 65 \\
\hline 2.5 & -100 & 40 & -66 & -49 \\
\hline
\end{tabular}


TableB 9: KPI Evaluation KPI Turnaround time, Contractor Develops, working/proposed

\begin{tabular}{|c|c|c|c|c|c|}
\hline \multirow{2}{*}{ 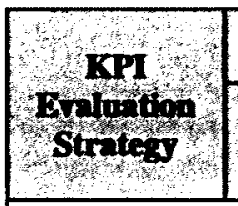 } & \multicolumn{5}{|c|}{ Why } \\
\hline & 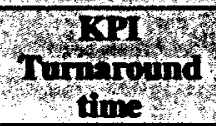 & 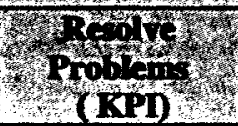 & 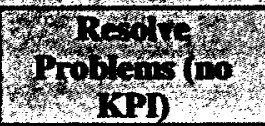 & Sylng & Than \\
\hline \multicolumn{6}{|c|}{ Category A: working system } \\
\hline 2.1 & 61 & 76 & 61 & 90 & 90 \\
\hline$\overline{2.2}$ & 61 & 76 & 61 & 5 & -10 \\
\hline \multicolumn{6}{|c|}{ Category A : proposed system } \\
\hline 2.3 & 91 & 90 & 90 & -37 & 90 \\
\hline 2.4 & 90 & 909 & 90 & 90 & 90 \\
\hline 2.5 & 50 & 40 & -46 & 90 & -46 \\
\hline
\end{tabular}

TableB 10: KPI Evaluation KPI Cost to correct, test, deploy and KPI Keep Min Cost working/proposed

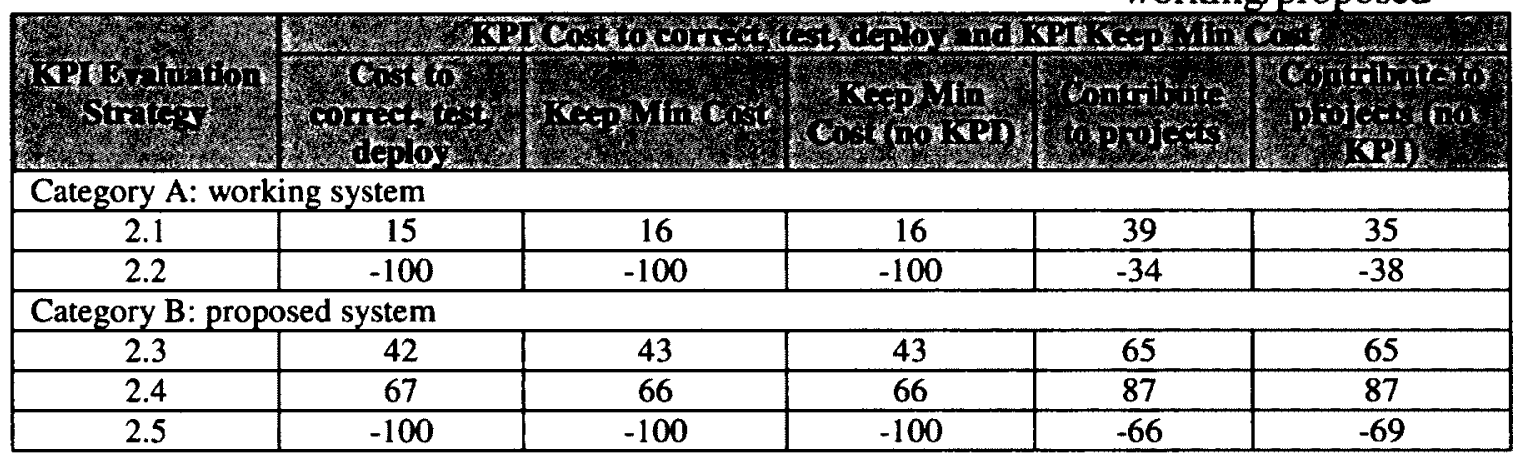

TableB 11 : Evaluation of Actors, Contractor Develops, working/proposed

\begin{tabular}{|c|c|c|c|}
\hline 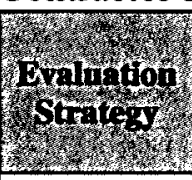 & 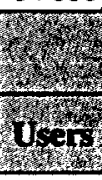 & 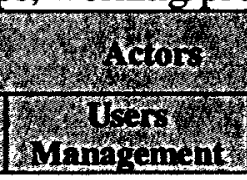 & Whand \\
\hline \multicolumn{4}{|c|}{ Category A: working system } \\
\hline 2.1 & 48 & 16 & 26 \\
\hline 2.2 & 11 & -100 & -28 \\
\hline \multicolumn{4}{|c|}{ Category B: proposed system } \\
\hline 2.3 & 77 & 43 & 48 \\
\hline 2.4 & 88 & 66 & 65 \\
\hline 2.5 & -20 & -100 & -51 \\
\hline
\end{tabular}

TableB 12: KPI Evaluation of Actors, Contractors Develop, working/proposed

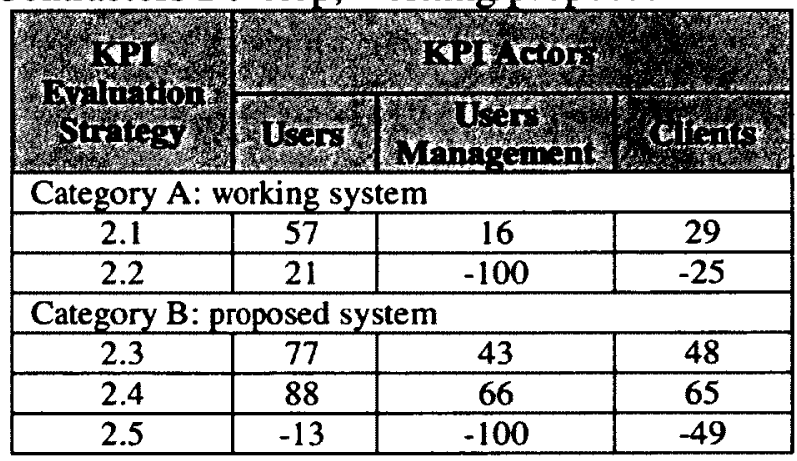

\section{Case 3c) Purchase product}

In Fig ....we have listed the strategies corresponding to this case; they relate to the

working system (from 3.1 to 3.2 ) and from (3.3 to 3.4 ) to the proposed system; the rest 
are the strategies corresponding to KPI for both types. We will execute the model for

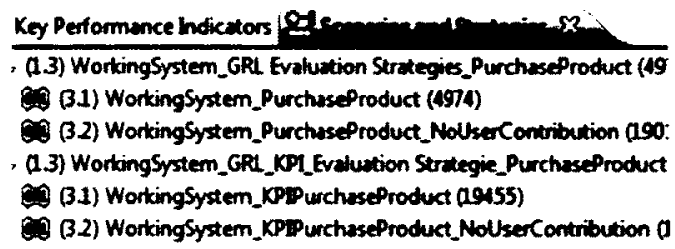

FigureB 18: Summary strategies Purchase Product

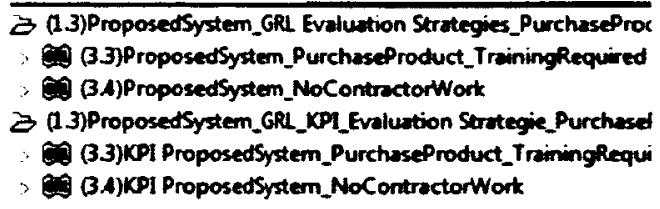

FigureB 19:Summary KPI strategies Purchase Product.

each strategy and acknowledge the evaluation results generated in accordance to the

characteristics of the strategies, including components and contributions.

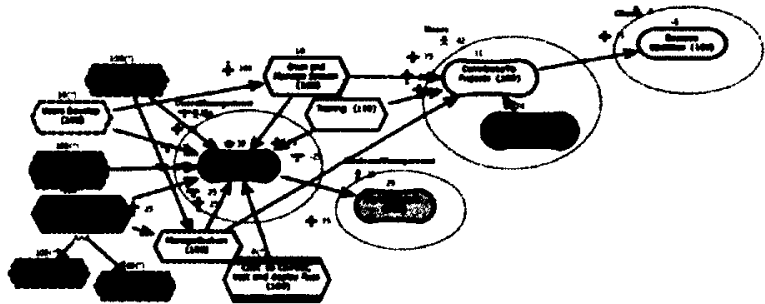

FigureB 20: Evaluation high-level diagram,

Purchase Product, Strategy (3.1)

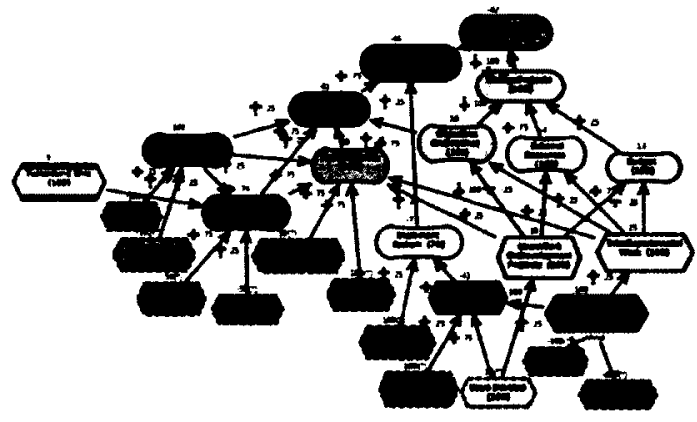

FigureB 22 : Evaluation of detailed graph,

Purchase Product, Strategy (3.1)

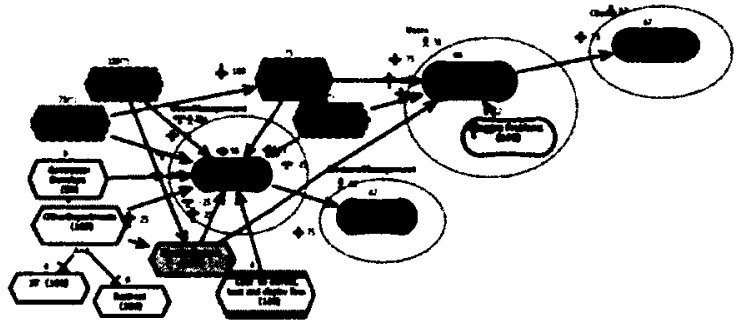

FigureB 21: Evaluation high-level diagram, Purchase Product, Strategy (3.3)

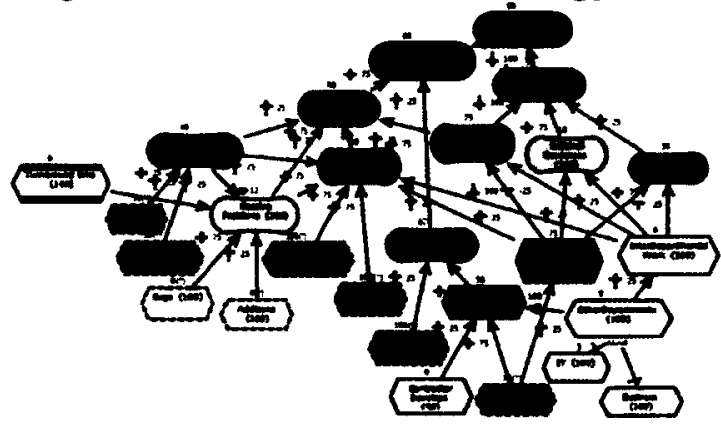

FigureB 23: : Evaluation of detailed graph, Purchase Product, Strategy (3.3)

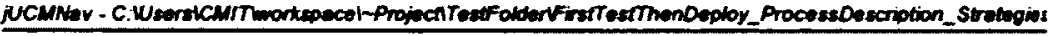

Strategy Legond for Group "(1.3) WorkingSysten_GRL_KPI_Evaluetion Strategle_PurchasePrc 1:(3.1) Wortingsystem_KPIPurchaseproduct

2:(3.2) WortingSyetem_KPIPurchaeeProduct_NolberContribution

FigureB 24: Extras from pdf report 
TableB 13:Evaluation of KPI Turnaround time and Maintain System, Contractor Develops, working proposed

\begin{tabular}{|c|c|c|c|c|c|c|}
\hline 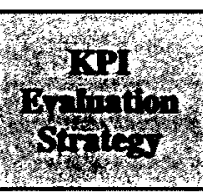 & 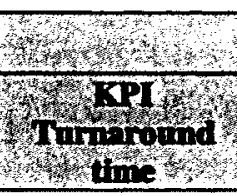 & 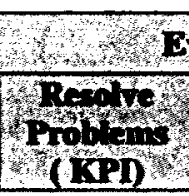 & 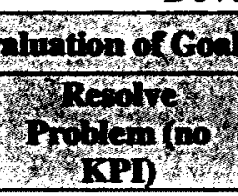 & Whing & & 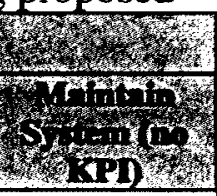 \\
\hline \multicolumn{7}{|c|}{ Category A: working system } \\
\hline 3.1 & -74 & -92 & -74 & -79 & & -61 \\
\hline 3.2 & -58 & -88 & -74 & -100 & & -100 \\
\hline \multicolumn{7}{|c|}{ Category B: proposed system } \\
\hline 3.3 & 33 & 20 & 12 & 90 & & 90 \\
\hline 3.4 & -5 & 2 & 3 & -55 & & -64 \\
\hline
\end{tabular}

TableB 14: KPI Evaluation for KPI Cost to correct, and KPI Keep Min Cost, Purchase Product, working/proposed Evaluati

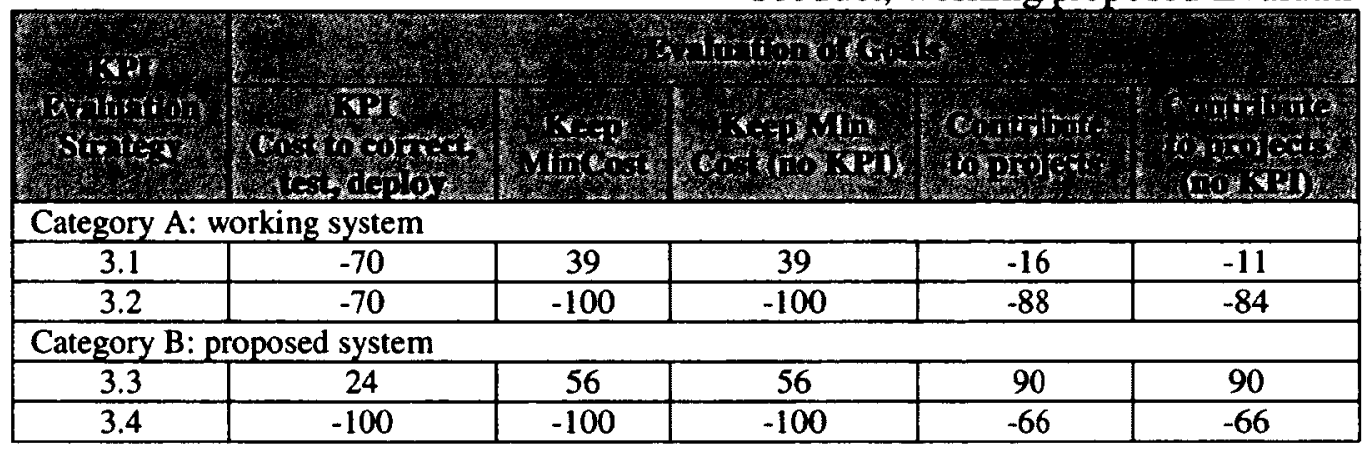

TableB 15: Evaluation of Actors, Purchase Product, working/purchase

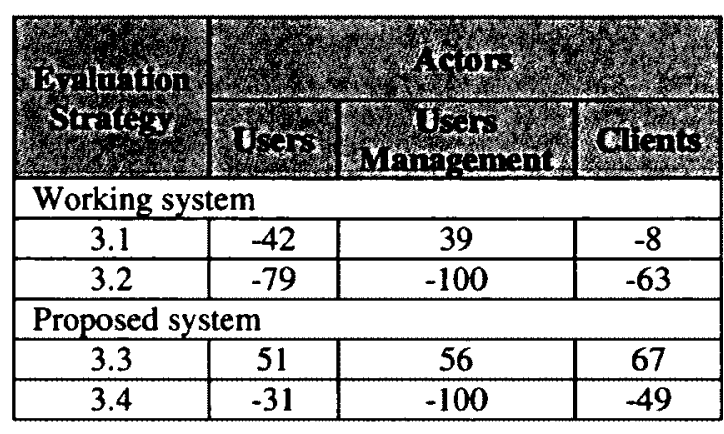

TableB 16: KPI Evaluation of Actors, Purchase Product, working/purchase

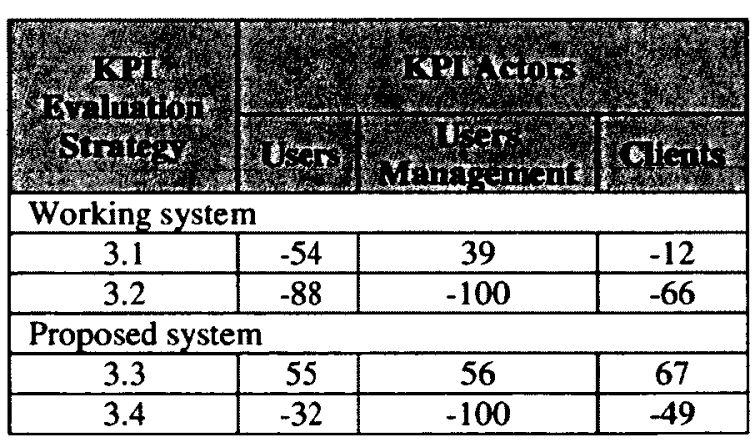

TableB 17: Evaluation of goals per Actors, Purchase Product, working/proposed

\begin{tabular}{|c|c|c|c|c|}
\hline $\sin (2+10)$ & 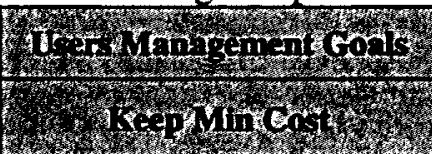 & 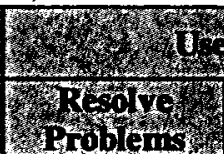 & 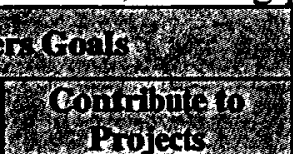 & 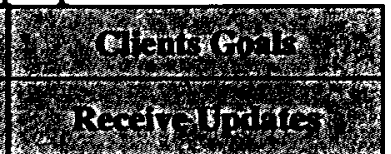 \\
\hline \multicolumn{5}{|c|}{ Category A: working system } \\
\hline 3.1 & 39 & -74 & -11 & -8 \\
\hline 3.2 & -100 & -74 & -84 & -63 \\
\hline \multicolumn{5}{|c|}{ Category B: proposed system } \\
\hline 3.3 & 56 & 12 & 90 & 67 \\
\hline 3.4 & -100 & 3 & -66 & -49 \\
\hline
\end{tabular}


TableB 18: KPI Evaluation of goals per Actors, Purchase Product, working/proposed

\begin{tabular}{|c|c|c|c|c|}
\hline 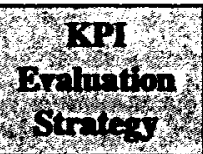 & 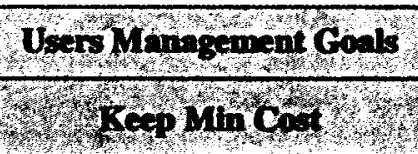 & 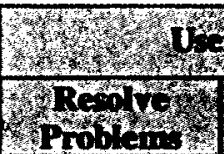 & 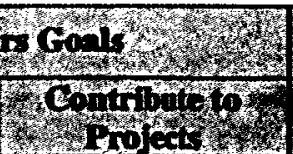 & W \\
\hline \multicolumn{5}{|c|}{ Category A: working system } \\
\hline 3.1 & 39 & -92 & -16 & -12 \\
\hline 3.2 & -100 & -88 & -88 & -66 \\
\hline \multicolumn{5}{|c|}{ Category B:proposed system } \\
\hline 3.3 & 56 & 20 & 90 & 67 \\
\hline 3.4 & -100 & 2 & -66 & -49 \\
\hline
\end{tabular}

\section{Case 4c) Users develop}

In Figure B.25 and Figure B.26 we have listed the strategies corresponding to this case; they relate to the working system such as (from 4.1 to 4.3 ) and (4.4) to the proposed system; the rest are the strategies corresponding to KPI for both types. We will execute the model for each strategy and acknowledge the evaluation results generated in accordance to the characteristics of the strategies, including components and contributions. The results are displayed in Tables below.

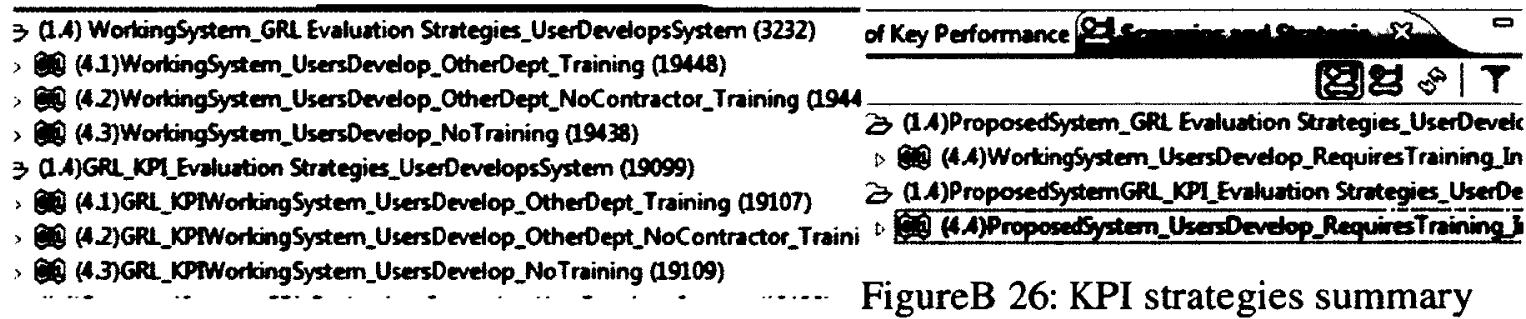

FigureB 25: Strategies summary, User develops

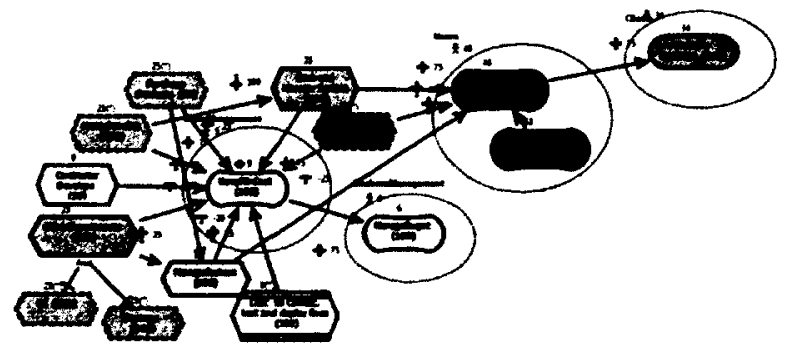

FigureB 27: Evaluation, User develops, Strategy (4.2)

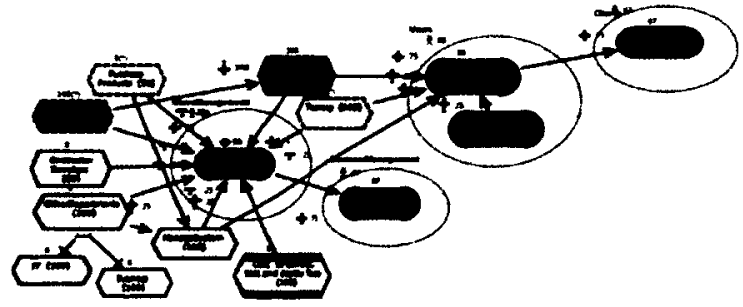

FigureB 28: Evaluation of high level graph, Users develop, Strategy (4.4) 


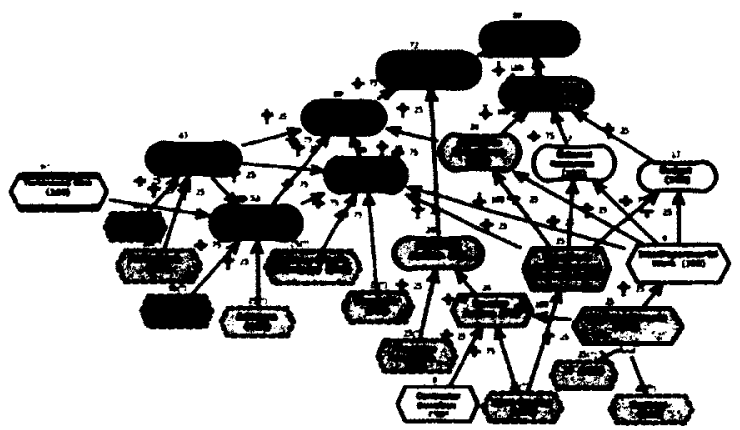

FigureB 29: Evaluation of detailed graph, Users develop, Strategy (4.2)

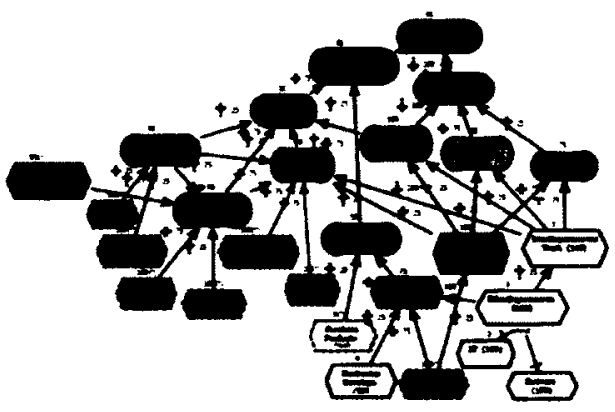

FigureB 30: Evaluation of detailed graph, Users develop, Strategy (4.4)

TableB 19: KPI Evaluation of goals with KPI Turnaround time, Users develop, working/proposed

\begin{tabular}{|c|c|c|c|c|c|}
\hline 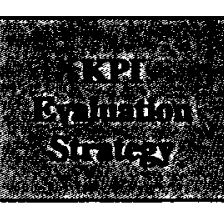 & 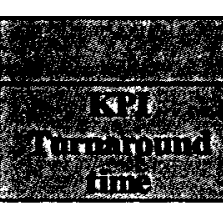 & 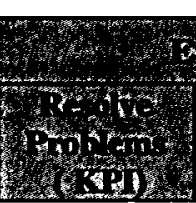 & 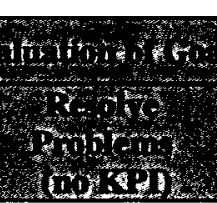 & 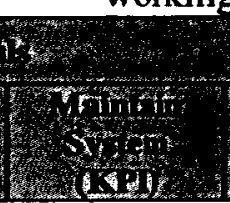 & 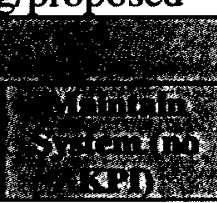 \\
\hline \multicolumn{6}{|c|}{ Category A: working system } \\
\hline 4.1 & 29 & 19 & 12 & 40 & 33 \\
\hline 4.2 & 75 & 71 & 53 & 90 & 89 \\
\hline 4.3 & 91 & 90 & 90 & 90 & 90 \\
\hline \multicolumn{6}{|c|}{ Category B: proposed system } \\
\hline 4.4 & 95 & 90 & 90 & 90 & 90 \\
\hline
\end{tabular}

TableB 20: KPI Evaluation of KPICost to correct, Keep Min Cost, Users develop, working/proposed

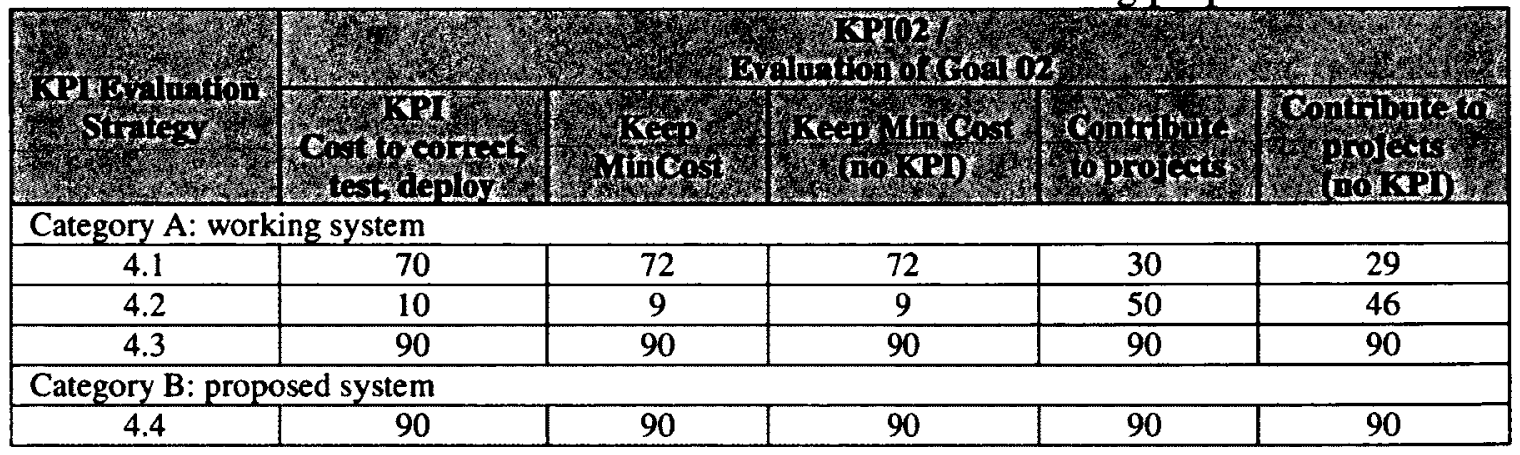


TableB 21: Evaluation of Actors, Users develop

\begin{tabular}{|c|c|c|c|}
\hline Boluntiog & bert & 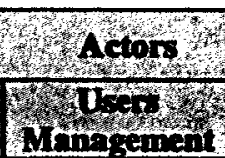 & 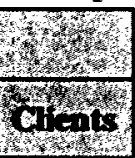 \\
\hline \multicolumn{4}{|c|}{ Category A: working system } \\
\hline 4.1 & 20 & 72 & 21 \\
\hline 4.2 & 49 & 9 & 34 \\
\hline 4.3 & 90 & 90 & 67 \\
\hline \multicolumn{4}{|c|}{ Category B: proposed system } \\
\hline 4.4 & 90 & 90 & 67 \\
\hline
\end{tabular}

TableB 22: KPI Evaluation of Actors, Users develop

\begin{tabular}{|c|c|c|c|}
\hline 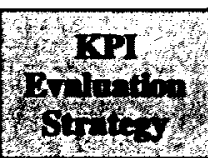 & 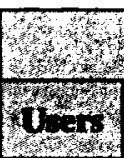 & 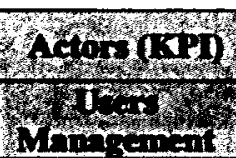 & 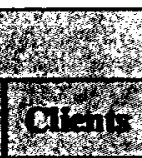 \\
\hline \multicolumn{4}{|c|}{ Category $\mathrm{A}$ : working system } \\
\hline 4.1 & 24 & 72 & 22 \\
\hline 4.2 & 60 & 9 & 37 \\
\hline 4.3 & 90 & 90 & 67 \\
\hline \multicolumn{4}{|c|}{ Category B: proposed system } \\
\hline 4.4 & 90 & 90 & 67 \\
\hline
\end{tabular}

TableB 23: Evaluation of goals foe Actors (UM, Users, Clients), Users develop, working/proposed system

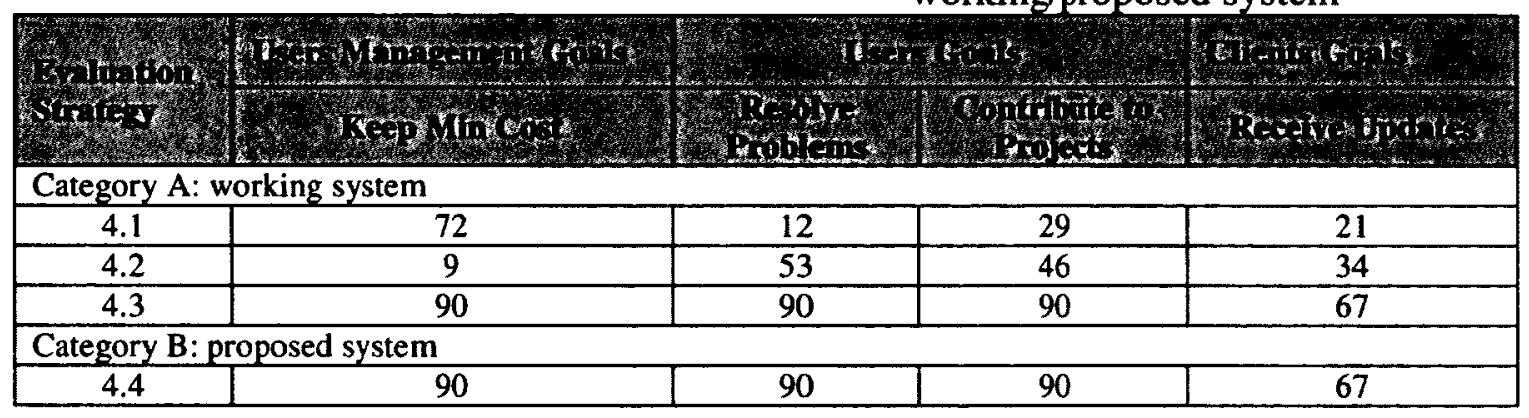

TableB 24: KPI Evaluation of Actors goals (UM, Users, Clients)

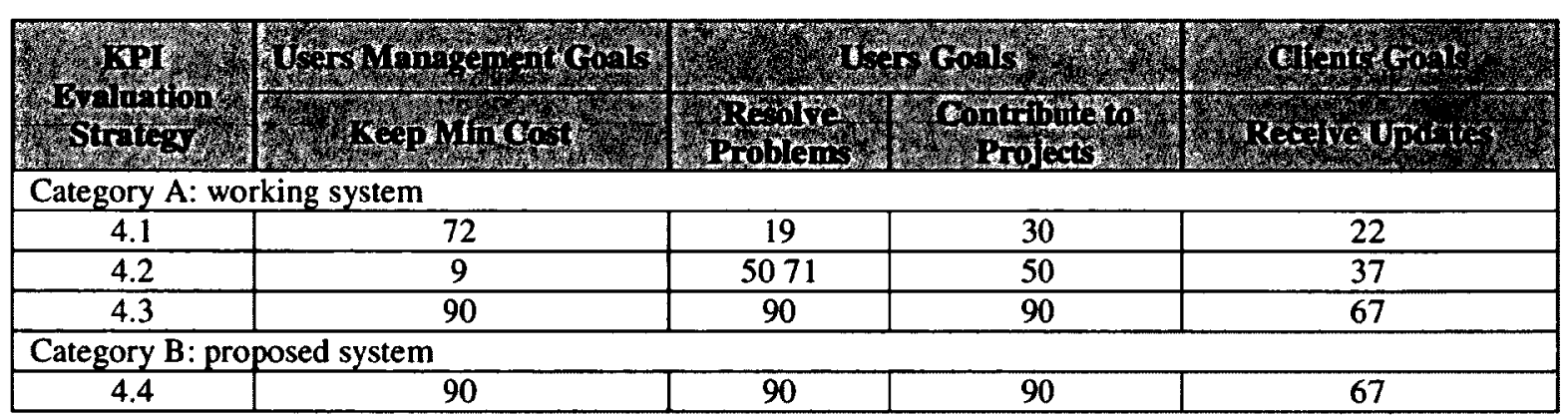




\section{Appendix C - System Monitoring}

\section{Case working system}

The system implemented in the Users SO, and utilized for daily work, is part of the complex distributed system installed across the organization and shared by all component SO. The IT SO is in charge with the system management at the organization level, as well as at departmental level, managing systems for the majority of the SO; the staff from ITD and ITM SO "deals with such issues as program and project management, technical direction of development, quality management, configuration management, and risk management" [Sage01] ensuring "to minimize network failure and malfunctioning" [Sladic11]. System monitoring should be capable of ensuring "a high percentage of data recovery in a long time"; "should also have the ability to prevent computer virus attacks or take measures to ensure there is no error in system's data storage and transmission process" [Quan 11]. In few cases, there are SO capable of owning and administering their own systems, using own staff to manage information technology services. Whereas in our use case, the ITD is managing and monitoring system and network services and technologies, while the BD is managing and monitoring the business aspects of the system, including business requirements documentation. With respect to the centralization of information and services process, we understand that is important to achieve goals at the organizational level. We have also learned from experience and studies [Zheng06], [Sladic11] that the realization of objectives at a high level is constructed on the realization of distinct goals per SO, while it depends on the satisfaction level of each goal, capability of mitigating potential conflicting goals, interference of responsibilities, identification of the impacts of other SO's goals in the 
realization of objectives of other departments, distribution of the same human resource on departmental projects at the SO level, as well on inter-departmental projects implemented across the organization and with external partners, and last but not least, on budget. And with the rapid growth of technology and development of services at organizational level, studies [Zheng06], [Sladic11] are raising awareness that "due to a lack of support for standardized monitoring and management protocols, heterogeneity of network components, ever-increasing network deployment, and rapid development of new network technologies, flexible mechanisms to monitor network are highly required", recommending beneficial to have a "network control and decision process layer to describe the time delays in both the communications network and the decision processes that control organizational response" [Zheng06].From the Users point of view, we believe that it will be beneficial for all $\mathrm{SO}$, as well as for the whole organization, if the monitoring process (described in Fig below from the users point of view) will be periodically modeled and simulated, to ensure that the goals and objectives that belong to each SO are preserved and achieved with the satisfaction levels planned by each SO. proceed with the analysis and evaluation of the model goals, we present the two model diagrams, high-level view (as seen in FigureC4) and FigureC5) as a reflection of the monitoring process seen by the users point of view, even though with limited access to monitoring process and information.

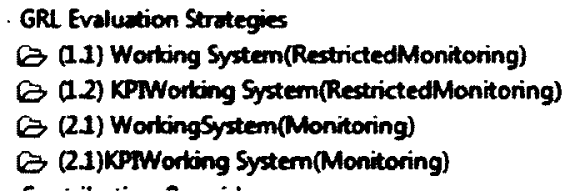

FigureC 1: Summary strategies

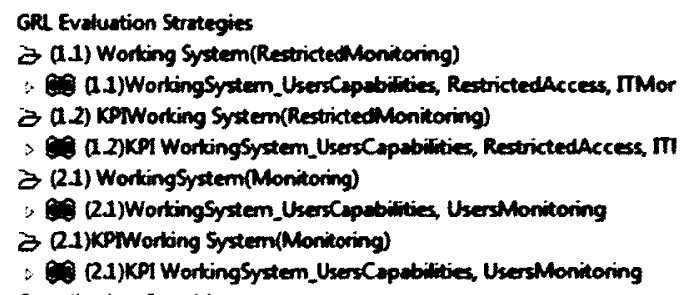

FigureC 2: Strategies details 
We are proposing two strategies for this case (FigureC1 and FigureC2 with KPI) in which the Users have limited monitoring capabilities, as in the real case, or have monitoring capabilities, as it would be deisred. After we execute each strategy, we will have a look at the evaluation results, that are dsplayed above each element in the model, or that could be exported in .pdf or .csv (as seen in Figure C3) reports.

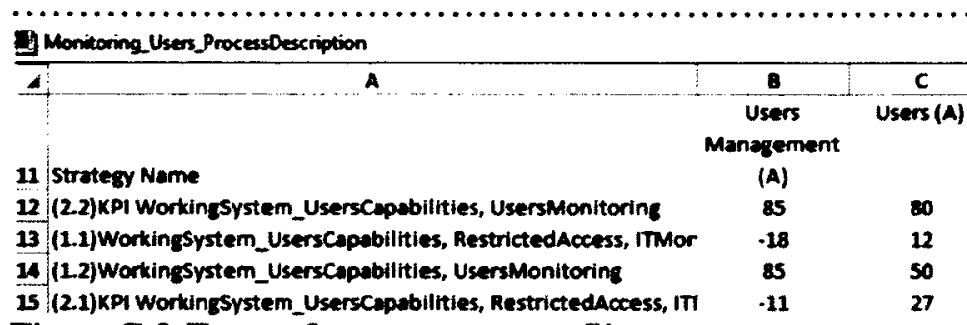

FigureC 3:Extras from .csv report file

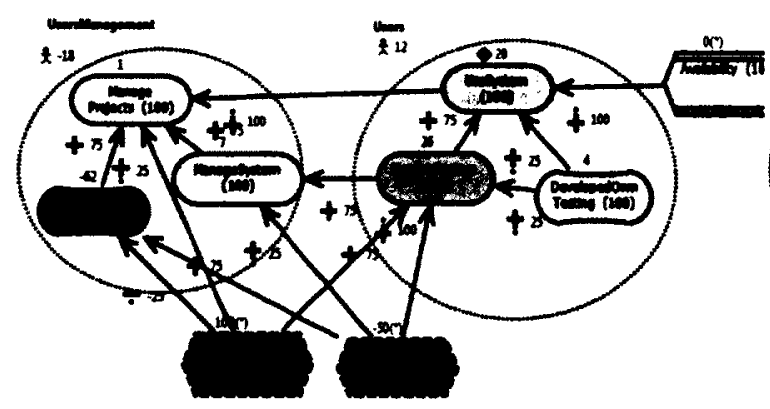

FigureC 4: Evaluation of high-level diagram, Monitoring system, Strategy (1.1)

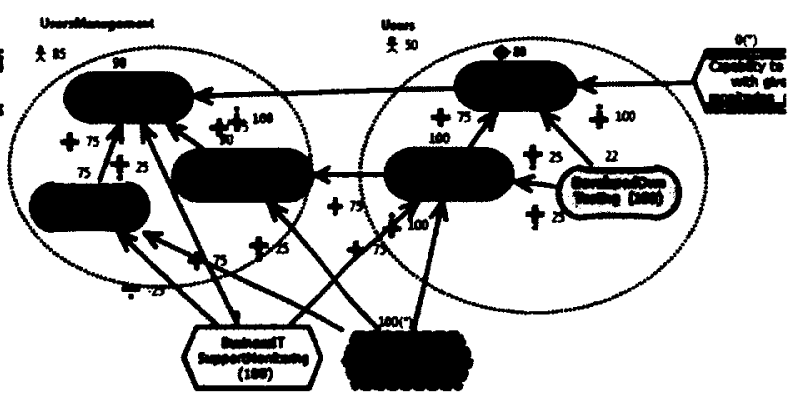

FigureC 5: Evaluation of high-level diagram, Monitoring system, Strategy (1.2) 


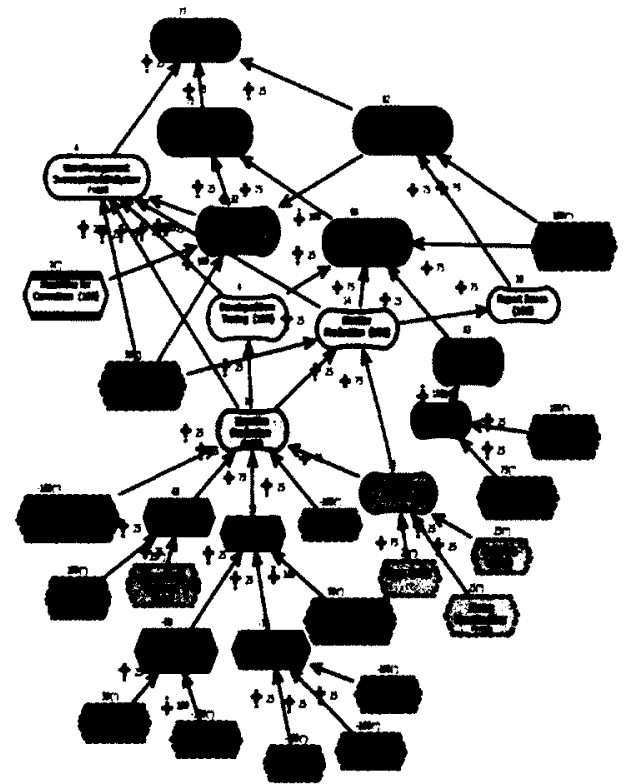

FigureC 6: Evaluation of Strategy (1.2)

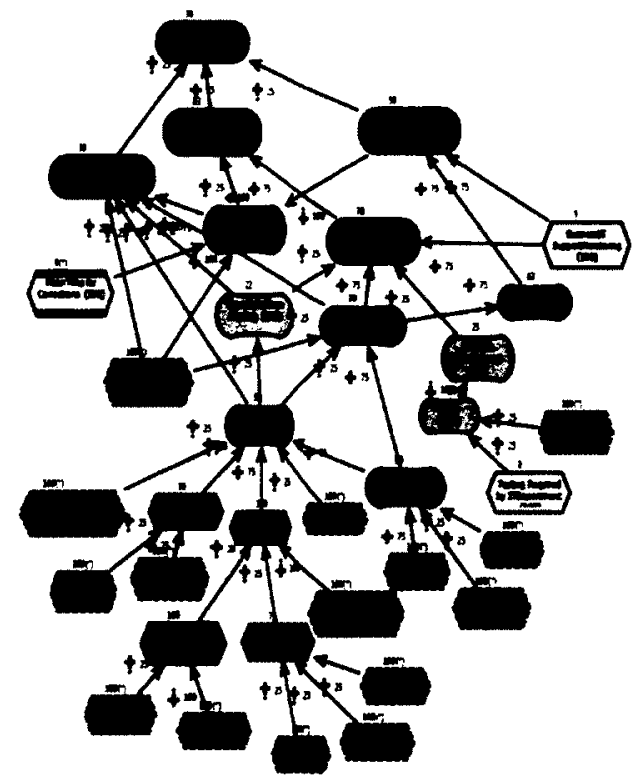

FigureC 7: Evaluation of Strategy (1.2)

After the execution, we analyze the results of the GRL evaluation, for each strategy (as seen in Figure C6 and Figure C7). As expected, we can see that the goals will have different realizations as presented in the tables to follow.

TableC 1: Evaluation of Actors

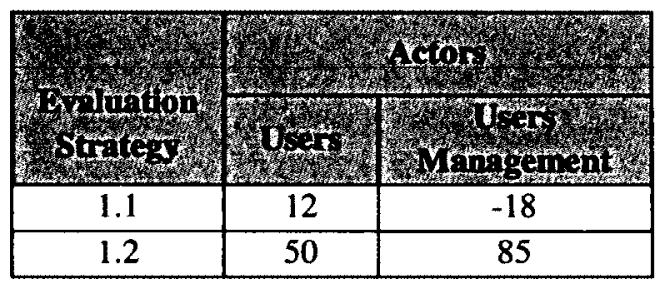

TableC 2: Evaluation of goals

\begin{tabular}{|c|c|c|}
\hline 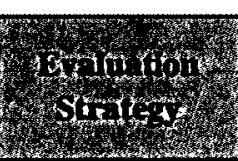 & 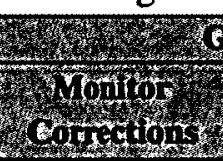 & 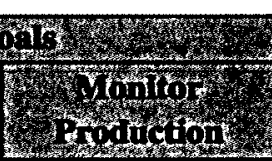 \\
\hline 1.1 & 32 & 14 \\
\hline 1.2 & 100 & 90 \\
\hline
\end{tabular}

TableC 3: Evaluation of goals per Actors

\begin{tabular}{|c|c|c|c|c|c|}
\hline Sirotity & 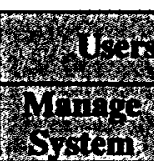 & 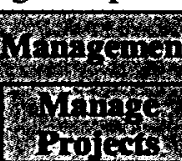 & High & 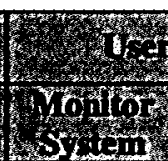 & 6rits \\
\hline 1.1 & 7 & 1 & -62 & 26 & 20 \\
\hline 1.2 & 90 & 90 & 75 & 100 & 80 \\
\hline
\end{tabular}

We work with two KPIs in this model 'Mean Time for Correction' and 'Availability': 
- Mean Time for Correction - represents the average time required to resolve an issue, a component, application, system module that failed or is not available. It is the sum of correction times divided by the sum of the number of corrective actions completed over a given period (one year). Given today's technology and capabilities, we set the Target value $24 \mathrm{~h}$, the Treshhold to $72 \mathrm{~h}$ ( 3 days/year the system could be down) and Worst value, to 120 hours ( 5 days). The correction period of time excludes the maintenance work, etc

- 'Availability'- is defined as the capability of the users to connect, work on the system. It is calculated as the system's working time (uptime expressed in percentage) per year. Due to performant technology, the potential uptime could be set 365 days (knowing that servers hosting applications and the system could automatically switch on back-up servers in case of system failure. To be realistic, for such a case, we have estimate that three days could be assigned for IT related work on the system; therefore the Availability would be $(365-3) /[(365-3)+3]=362 / 365$, with result 0.991780821917 rounded for a final result value of $99.178 \%$. In parallel, we have considered few other potential scenarios, such as: system down for five days, incomplete update queries used for a month (31days), deployment of new functionalities on the old system platform, which somehow have affected the users work for 41 days in a row, without their knowledge; this situation could lead to an availability of: $(365-3) /[(365-3)+41]=362 /(362+$ $41)=362 / 403=0.89826302729$ with result approximated to $\sim 89.8263$.

Due to space limitations, we presented samples from the KPI evaluation results, as seen in the tables to follow; the results can be exported in pdf and csv files for further use. 
TableC 4: KPI Evaluation of Actors

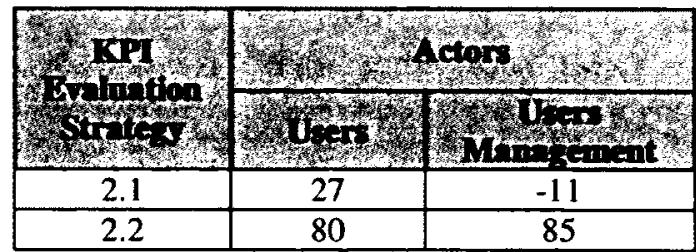

TableC 5: KPI evaluation with KPI Mean Time for Correction

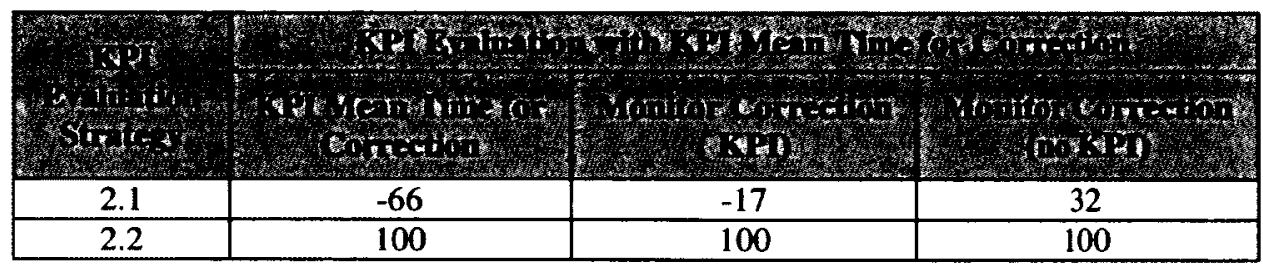

TableC 6: KPI Evaluation with KPI Availability

\begin{tabular}{|c|c|c|c|c|c|}
\hline 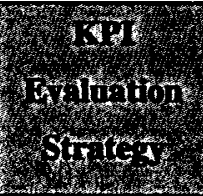 & Writing & 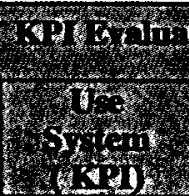 & 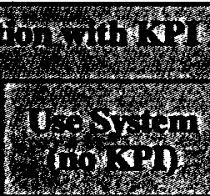 & 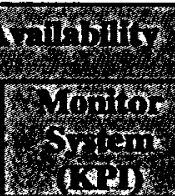 & 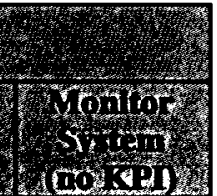 \\
\hline 2.1 & 29 & 49 & 20 & 26 & 26 \\
\hline 2.2 & 100 & 100 & 80 & 100 & 100 \\
\hline
\end{tabular}

TableC 7: KPI Evaluation of UM Goals

\begin{tabular}{|c|c|c|c|c|c|c|c|}
\hline 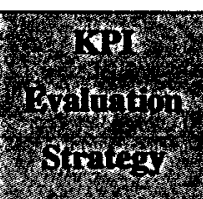 & $y^{4}$ & $\frac{6}{(k+1)}$ & 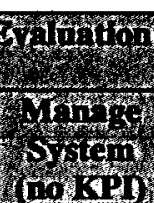 & 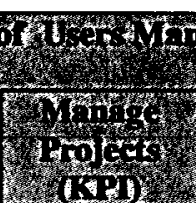 & 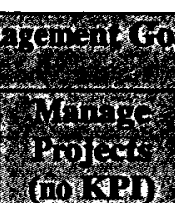 & 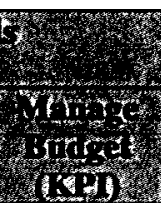 & 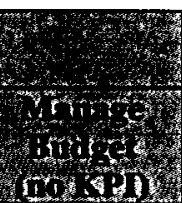 \\
\hline 2.1 & 29 & 7 & 7 & 22 & 1 & -62 & -62 \\
\hline 2.2 & 100 & 90 & 90 & 90 & 90 & 75 & 75 \\
\hline
\end{tabular}

We continue the Performance analysis by presenting you with the overall satisfaction for all KPIs per strategy, indicating the evaluation details per KPI, in the next paragraphs/

\section{Case proposed system}

In this case too we expect to have close results with the results obtained for the best strategy from working system, given that the Users have monitoring access and 
capabilities to track all the information on system behaviour, functionality and activities they need. We used the same type of strategies as in the working system case, and the evaluation results are presented in the manner in the tables that follow.

TableC 8: KPI Evaluation of Actors (proposed system)

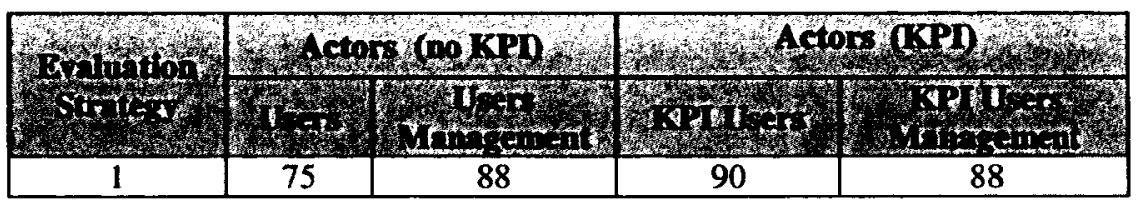

TableC 9: Evaluation of UM goals (proposed system)

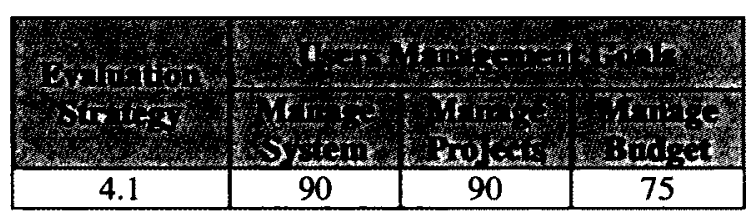

TableC 10: KPI Evaluation of UM's goals (proposed system)

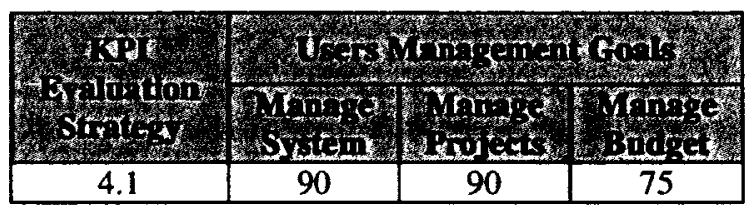

TableC 11: KPI Evaluation of goals with KPI Availability and KPI Mean Time for Correction (proposed system)

\begin{tabular}{|c|c|c|c|c|c|c|}
\hline 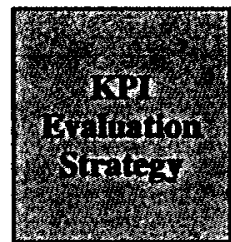 & (2) & 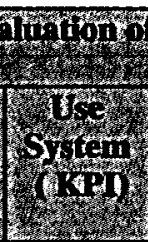 & 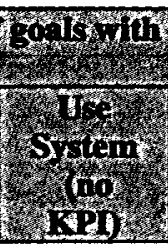 & 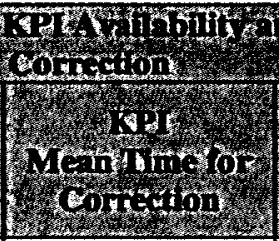 & (1) & 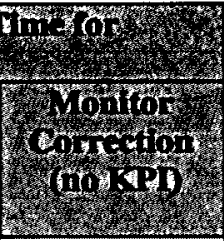 \\
\hline 4.1 & 100 & 90 & 75 & 75 & 100 & 100 \\
\hline
\end{tabular}

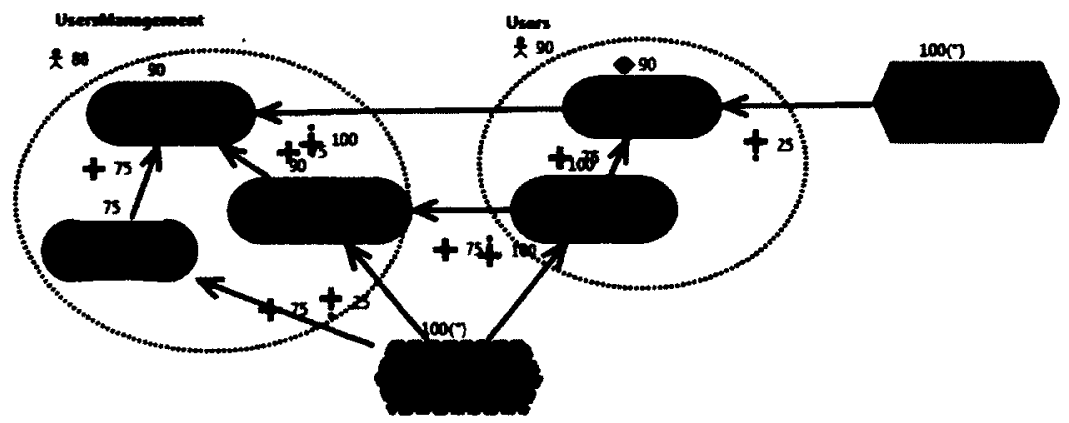

FigureC 8: Evaluation of high level diagram, results indicated in TableC11 (Strategy2.2) 


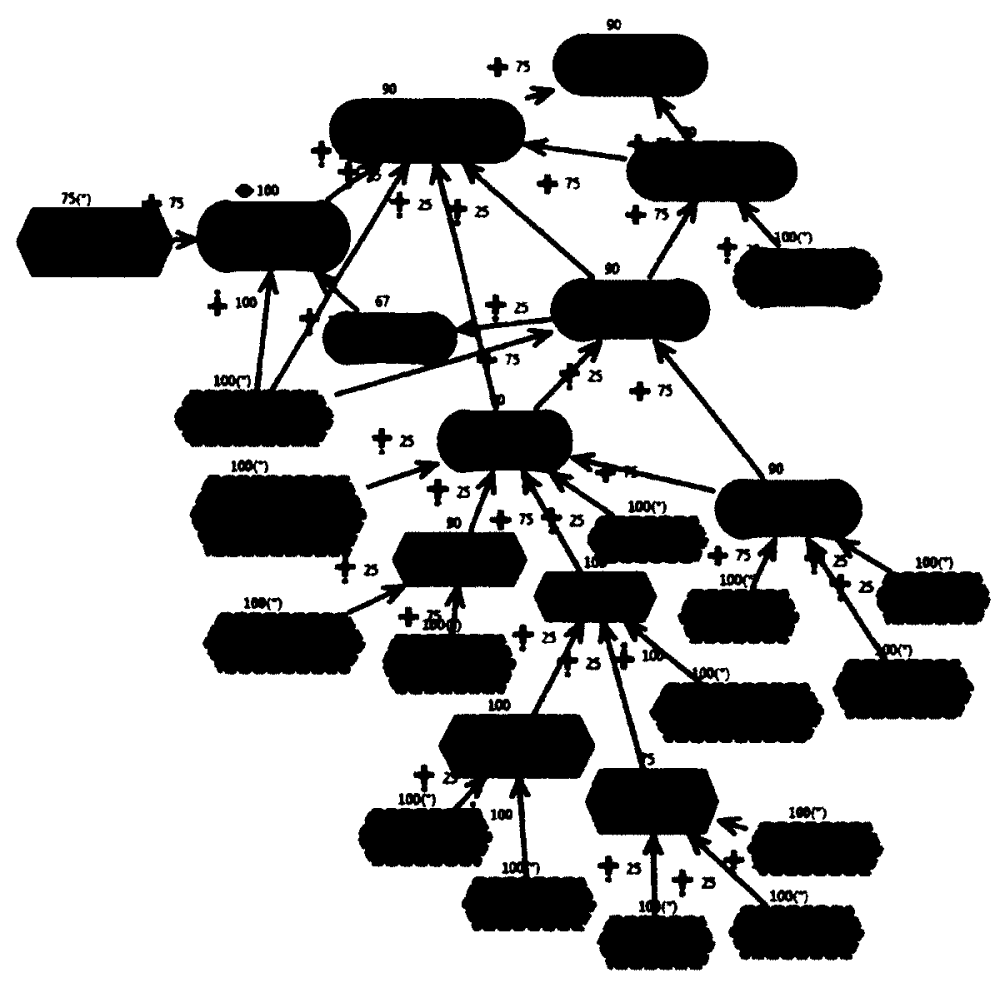

FigureC 9: Evaluation of detailed GRL Graph for proposed system (all goals realized close to satisficed level) 